\title{
MEASURED STEADY AND UNSTEADY AERODYNAMIC PERFORMANCE OF A FAMILY OF THREE HIGHLY-LOADED LOW-PRESSURE TURBINE CASCADES
}

by

\section{Ivan Popović}

\begin{abstract}
A thesis submitted to the Faculty of Graduate Studies and Research in partial fulfillment of the requirements for the degree of
\end{abstract}

\section{Master of Applied Science}

in Aerospace Engineering

Ottawa-Carleton Institute for Mechanical \& Aerospace Engineering

\author{
Department of Mechanical \& Aerospace Engineering \\ Carleton University \\ Ottawa, Ontario, Canada
}

(C)Ivan Popović

July 2005 
Library and

Archives Canada

Published Heritage

Branch

395 Wellington Street

Ottawa ON K1A 0N4

Canada
Bibliothèque et

Archives Canada

Direction du

Patrimoine de l'édition

395 , rue Wellington

Ottawa ON K1A 0N4

Canada

Your file Votre référence

ISBN: 978-0-494-44026-1

Our file Notre référence

ISBN: 978-0-494-44026-1

NOTICE:

The author has granted a nonexclusive license allowing Library and Archives Canada to reproduce, publish, archive, preserve, conserve, communicate to the public by telecommunication or on the Internet, loan, distribute and sell theses worldwide, for commercial or noncommercial purposes, in microform, paper, electronic and/or any other formats.

The author retains copyright ownership and moral rights in this thesis. Neither the thesis nor substantial extracts from it may be printed or otherwise reproduced without the author's permission.
AVIS:

L'auteur a accordé une licence non exclusive permettant à la Bibliothèque et Archives Canada de reproduire, publier, archiver, sauvegarder, conserver, transmettre au public par télécommunication ou par l'Internet, prêter, distribuer et vendre des thèses partout dans le monde, à des fins commerciales ou autres, sur support microforme, papier, électronique et/ou autres formats.

L'auteur conserve la propriété du droit d'auteur et des droits moraux qui protège cette thèse. $\mathrm{Ni}$ la thèse ni des extraits substantiels de celle-ci ne doivent être imprimés ou autrement reproduits sans son autorisation.
In compliance with the Canadian

Privacy Act some supporting forms may have been removed from this thesis.

While these forms may be included in the document page count, their removal does not represent any loss of content from the thesis.
Conformément à la loi canadienne sur la protection de la vie privée, quelques formulaires secondaires ont été enlevés de cette thèse.

Bien que ces formulaires aient inclus dans la pagination, il n'y aura aucun contenu manquant.

\section{Canadä}




\section{Abstract}

Midspan measurements were made in a low-speed wind tunnel for three lowpressure turbine (LPT) cascades at design and off-design incidence. The baseline profile was the midspan section of an LPT vane of a Pratt \& Whitney Aircraft (PWA) engine, and is regarded as moderately highly-loaded blade. To examine the influence of increased loading two additional airfoils were designed by PWA, each providing $25 \%$

higher loading than the baseline version. All three airfoils have the same axial chord, and inlet and outlet design flow angles. The main difference between the airfoils with higher loading was in the pressure distribution: one airfoil was front-loaded, while the other was aft-loaded.

The aerodynamic performance was investigated for wide range of Reynolds numbers at design incidence and for two values of freestream turbulence intensity. In addition, the performance at $+5^{\circ}$ and $-5^{\circ}$ relative to the design incidence was examined at the same two turbulence intensities for a fixed value of Reynolds number. Measurements were made using a three-hole pressure probe, surface pressure taps and single hotwire probe. For one of the low Reynolds number cases, the unsteady flow effects were examined by using a moving-bar wake generator.

It was found that an increase of $25 \%$ in loading was possible only with a small increase in the losses. The front loaded airfoil performed much better at both low Reynolds numbers and at off-design incidence than the aft loaded one due to milder pressure gradients on the suction surface of the airfoil. The effects of the wakes generated by a simulated upstream blade row were beneficial for the stalled case, resulting in flow reattachment and a consequent reduction in losses. The upstream wakes resulted in a slight loss increase for the other case where the flow was attached. 


\section{Acknowledgements}

Any study, especially an experimental study such as this one, cannot be accomplished without help and support of many. First I would like to thank my supervisor, Professor Steen Sjolander, for his guidance, assistance and advices throughout the course of this and previous studies.

I would also like to thank my colleagues and true friends: Dr. Ali Mahallati, Dr. Junqiang Zhu and Ms. Tatjana Zorić for their unselfish help and encouragements. Special thanks are extended to Mr. Alex Proctor, Mr. Steve Truttmann and all supporting staff of the machine shop. Finally, I have to mention Mrs. Nancy Powell, Mrs. Christie Egbert and Mrs. Marlene Groves who over the years helped me on numerous occasions navigating through sometimes dark waters of bureaucracy and to whom I will be always grateful for that.

This marks the end of a long journey since I first came to Carleton University, and the list of all people who in one way or another either helped me or made this journey more bearable would be long. I hope that those not mentioned will forgive me; however, they can be sure that I won't forget them. The financial support of Pratt \& Whitney Aircraft (East Hartford, CT) is also acknowledged. 


\section{Table of Contents}

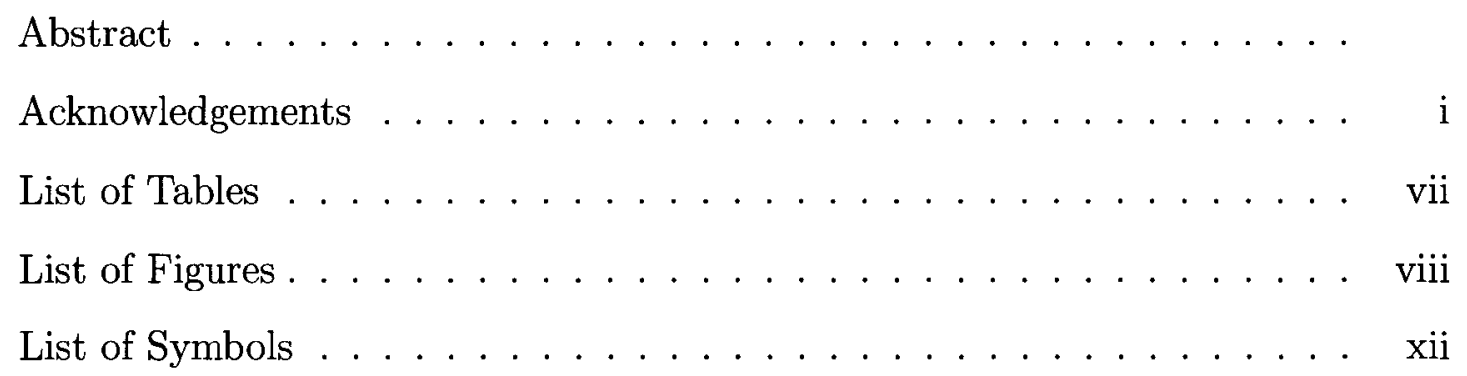

1 Introduction $\quad 1$

2 Literature Review 5

2.1 Introduction . . . . . . . . . . . . . . 5

2.2 Nomenclature and Terminology . . . . . . . . . . . . . . 6

2.2.1 Airfoil Nomenclature . . . . . . . . . . . . . 6

2.2.2 Loss Breakdown Terminology . . . . . . . . . . . . . . 6

2.3 Zweifel Coefficient. . . . . . . . . . . . . . . 9

2.4 Modes of Transition and Laminar Separation Bubbles . . . . . . . . . 10

2.4.1 Modes of Transition . . . . . . . . . . . . . 10

2.4.2 Laminar Separation Bubbles . . . . . . . . . . . . . . . 13

2.5 Profile Loss Generation Mechanisms . . . . . . . . . . . . . . 15

2.6 Overview of Unsteady Flow and its Influence on Losses . . . . . . . 19

2.7 Summary of Experimental Investigations . . . . . . . . . . . . . 22 
2.8 Empirical Profile Loss Prediction Methods . . . . . . . . . . . . . 27

3 Experimental Apparatus $\quad 35$

3.1 Introduction . . . . . . . . . . . . . . . 35

3.2 Low-Speed Wind Tunnel . . . . . . . . . . . . . . . . . . 36

3.2 .1 Wind Tunnel . . . . . . . . . . . . . 36

3.2 .2 Test Section . . . . . . . . . . . . . . 37

3.2 .3 Test Cascades . . . . . . . . . . . . . . . . 39

3.2.4 Turbulence Generating Grids . . . . . . . . . . . . . . 40

3.2 .5 Wake Generator Rig . . . . . . . . . . . . . 43

3.3 Instrumentation and Probes . . . . . . . . . . . . . . . 44

3.3.1 Pressure Transducers . . . . . . . . . . . . . . 44

3.3.2 Temperature Monitor . . . . . . . . . . . . . . 46

3.3.3 Digital Barometer . . . . . . . . . . . . . . 46

3.3 .4 Hotwire Anemometry . . . . . . . . . . . . . . . 47

3.3.5 Three-hole Probe . . . . . . . . . . . . . . 47

3.3.6 Single-Wire Hotwire Probe . . . . . . . . . . . . . . . . . 49

3.4 Data Acquisition System . . . . . . . . . . . . . . . 49

3.5 Calibration Apparatus and Methods . . . . . . . . . . . . 50

3.5.1 Pressure Transducer Calibration . . . . . . . . . . . 50

3.5.2 Calibration Apparatus . . . . . . . . . . . . 51

3.5.3 Three-hole Probe Calibration Procedure . . . . . . . . . . 52

3.5.4 Single-Wire Hotwire Calibration . . . . . . . . . . . . 53

4 Experimental Procedures and Data Reduction 55

4.1 Introduction . . . . . . . . . . . . . . . 55

4.2 Experimental Procedures and Data Reduction for Steady Flow Measurements ...................... 56 
4.2.1 Wind Tunnel Operating Point . . . . . . . . . . . . 56

4.2 .2 Three-hole Probe Measurements . . . . . . . . . . . . . . 57

4.2.3 Inlet Flow Uniformity and Outlet Flow Periodicity _. . . . 59

4.2.4 Two Dimensionality of the Midspan Flow . . . . . . . . . 64

4.2.5 Mixed-out Profile Loss and Flow Angle . . . . . . . . . . 65

4.2 .6 Blade Loading Measurements . . . . . . . . . . . 67

4.3 Data Reduction for Periodically-Unsteady Flow Measurements . . . . 69

4.3 .1 Ensemble Averaging . . . . . . . . . . . . . . . . . 69

4.3.2 Single Hotwire Measurements . . . . . . . . . . . . . 69

4.3 .3 Calculation of Bar Loss . . . . . . . . . . . . . . . . 71

4.3.4 Mixed-out Profile Loss . . . . . . . . . . . . . . . . . . 72

4.4 Uncertainty Analysis $\ldots \ldots \ldots \ldots \ldots \ldots$

5 Performance for Steady Flow at Design Incidence $\quad 78$

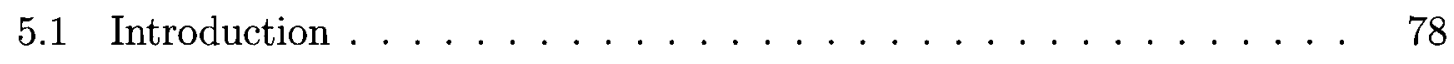

5.2 Comparison of Loadings at Design Conditions . . . . . . . . . 79

5.3 Reynolds Number Sensitivity of PAKD-F . . . . . . . . . . 82

5.3 .1 Loading Distributions at FSTI of $1.5 \% \ldots \ldots \ldots$

5.3.2 Influence of Higher FSTI (4\%) on Loading . . . . . . . 86

5.3 .3 Axial Velocity Ratios at Midspan _. . . . . . . . . 88

5.3.4 Mixed-out Profile Losses . . . . . . . . . . . . . . . . . . 89

5.3.5 Mixed-out Flow Angles . . . . . . . . . . . . . . . . . . . 91

5.4 Aerodynamic Comparison of PAKB and PAKD-A with PAKD-F . . . 92

5.4 .1 Loading Distributions at FSTI $(1.5 \%) \ldots \ldots \ldots \ldots$

5.4.2 Influence of Higher FSTI (4\%) on Loading . . . . . . . 97

5.4 .3 Mixed-out Profile Losses . . . . . . . . . . . . . . . . 99

5.4 .4 Mixed-out Flow Angles . . . . . . . . . . . . . . . . 101 
5.5 Summary of Design Incidence Performance . . . . . . . . . . . . 103

6 Performance for Steady Flow at Off-Design Incidence 105

6.1 Introduction . . . . . . . . . . . . . . . . 105

6.2 Loading Distributions . . . . . . . . . . . . . . . . . 107

6.2.1 Influence of Varying Incidence on Loading at FSTI of 1.5\% . . 107

6.2.2 Influence of Higher FSTI (4\%) on Loading . . . . . . . . 112

6.3 Axial Velocity Ratio . . . . . . . . . . . . . . . . . . . 114

6.4 Mixed-out Profile Losses . . . . . . . . . . . . . . . . . . . 115

6.4.1 Influence of Varying Incidence on Profile Losses . . . . . . 115

6.4.2 Influence of Higher FSTI (4\%) on Profile Losses . . . . . . 119

6.4.3 Comparison with Off-Design Correlations for Profile Losses . . 120

6.5 Mixed-out Flow Angles . . . . . . . . . . . . . . . . . . . . 122

6.6 Summary of Off-Design Performance Study . . . . . . . . . . 123

7 Performance for Periodically-Unsteady Flow at Design Incidence 125

7.1 Introduction . . . . . . . . . . . . . . . . . . . . . . 125

7.2 Reduced Frequency and Flow Coefficient . . . . . . . . . 126

7.3 Test Matrix . . . . . . . . . . . . . . . . . . . . 130

7.4 Mixed-out Profile Losses . . . . . . . . . . . . . . . . . . . . 131

7.4.1 The Effect of Varying Reduced Frequency on PAKD-A and PAKD-F . . . . . . . . . . . . . . . . 131

7.4.2 Comparison with Previous Measurements for PAKB . . . . . 135

7.5 Summary of Unsteady Flow Measurements . . . . . . . . . . . 137

8 Conclusions and Recommendations for Future Research 138

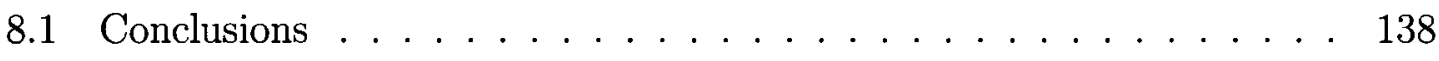

8.2 Recommendations for Future Research . . . . . . . . . . . . 143 
A Static Pressure Tap Locations

B Discussion of Static Pressure Coefficients for Loading Plots

C Time Variation of Losses for Unsteady Flow Measurements

163 


\section{List of Tables}

2.1 Summary of Previous Investigations $\ldots \ldots \ldots \ldots \ldots \ldots$

3.1 Summary of Airfoil Geometry . . . . . . . . . . . . . . . . . . 39

3.2 Summary of Turbulence Generating Grids . . . . . . . . . . . 42

3.3 Pressure Transducers . . . . . . . . . . . . . . . . . . . . 46

4.1 Summary of Pressure Tap Numbers for Loading Measurements . . . . 68

5.1 Summary of Test Cases Presented . . . . . . . . . . . . . . 79

5.2 Summary of Separation and Reattachment Points . . . . . . . . 85

6.1 Summary of Test Cases Presented . . . . . . . . . . . . . 106

A.1 Coordinates of Pressure Taps $\ldots \ldots \ldots \ldots \ldots$ 


\section{List of Figures}

2.1 Terminology of Linear Cascade Airfoils f . . . . . . . . . . . . . 7

2.2 Schematic of the Flow through a Turbine Blade Passage . . . . . 8

2.3 Schematic View of Attached Flow Transition Process . . . . . . 11

2.4 Schematic View of Separation Bubble . . . . . . . . . . . . . 14

2.5 Short vs. Long Separation Bubbles . . . . . . . . . . . . . . . 14

2.6 Dissipation Coefficient for Laminar and Turbulent Boundary Layers 16

2.7 Velocity Distribution . . . . . . . . . . . . . . . . 18

2.8 Profile Losses and Transition for a Various Reynolds numbers . . 19

2.9 Schematics of Stator-Rotor Interaction . . . . . . . . . . . . . 20

2.10 Profile Loss Variation under Unsteady Conditions . . . . . . . . 23

2.11 Profile Loss Variation under Unsteady Conditions for PAKB . . 23

2.12 A-M Loss Coefficients for Nozzle Blades . . . . . . . . . . . . 28

2.13 A-M Loss Coefficients for Impulse Blades . . . . . . . . . . . . . 28

3.1 Schematic of Low-Speed Open-Circuit Wind Tunnel . . . . . . 36

3.2 Isometric View of Test Section f . . . . . . . . . . . . . . . . 38

3.3 Comparison of airfoil geometry . . . . . . . . . . . . . . 41

3.4 Location of Turbulence Generating Grids . . . . . . . . . . . . . . 43

3.5 Schematic of Spoked-Wheel Wake-Generator Rig . . . . . . . . . 45 
3.6 Schematic of Three-Hole Probe . . . . . . . . . . . . . . 48

3.7 DRAL505DN Calibration Curves . . . . . . . . . . . 50

3.8 Schematic Drawing of Calibration Rig . . . . . . . . . . . . 51

3.9 Three-hole Probe Calibration Curves . . . . . . . . . . . . . . 54

3.10 Hotwire Calibration Curve . . . . . . . . . . . . . . 54

4.1 Locations of the Grids and Measurement Planes . . . . . . . . . . 61

4.2 Inlet Flow Uniformity and Outlet Flow Periodicity . . . . . . . . 62

4.3 Inlet Flow Uniformity and Outlet Flow Periodicity . . . . . . . 63

4.4 Measurement Planes and Nomenclature for Steady Flow . . . . . 66

4.5 Typical Exit Velocity Profiles for PAKD-F . . . . . . . . . . 70

4.6 Measurement Planes and Nomenclature for Unsteady Flow . . . 73

4.7 The losses for a Typical Unsteady Case . . . . . . . . . . . . 75

5.1 Comparison of Loadings at Design Conditions . . . . . . . . . . . 80

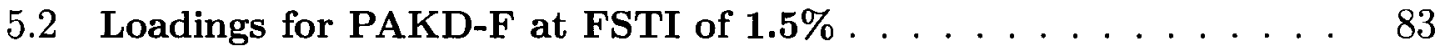

5.3 Comparison of Experimental and CFX 5 Computational Results 83

5.4 Bubble Growth on the Suction Side of PAKD-F at FSTI of 1.5\% 85

5.5 Loadings for PAKD-F at FSTI of $1.5 \%$ and $4 \% \ldots \ldots$

5.6 Axial Velocity Ratios for PAKD-F . . . . . . . . . . . . . . . . 88

5.7 Mixed-out Profile Losses for PAKD-F . . . . . . . . . . . . . . . . 90

5.8 Mixed-out Outlet Flow Angles for PAKD-F . . . . . . . . . . . . . 91

5.9 Comparison of Loadings at FSTI of 1.5\% . . . . . . . . . . . . . . 93

5.10 Bubble "Bursting" on PAKD-A at FSTI $=1.5 \% \ldots \ldots \ldots$

5.11 Comparison Between Loading Distributions for $1.5 \%$ and 4\% FSTI 98

5.12 Comparison of Profile Losses . . . . . . . . . . . . . . . . . 100

5.13 Comparison of Profile Losses with Correlations . . . . . . . . . . . 102 
5.14 Outlet Flow Angle Comparison . . . . . . . . . . . . . . . 103

6.1 Variation of $R e_{e}$ with Incidence . . . . . . . . . . . . . . 107

6.2 Comparison of Loadings at FSTI $=1.5 \% \ldots \ldots \ldots$

6.3 PAKD-A: Zweifel Coefficient and Flow Turning Variation . . . . 110

6.4 Bubble Growth on PAKD-F at $\mathrm{i}=\mathbf{- 5}^{\circ}$ and FSTI $=\mathbf{1 . 5 \%} \ldots \ldots$

6.5 Comparison of Loadings for Two Incidences and FSTIs . . . . . . 113

6.6 Axial Velocity Ratios at Midspan . . . . . . . . . . . . . . 115

6.7 Mixed-out Losses for FSTI $=\mathbf{1 . 5 \%} \ldots \ldots \ldots \ldots$

6.8 Main Contributors to Loss Production on the Suction Surface . . 117

6.9 Comparison of Mixed-out Losses for both FSTI Levels . . . . . . 119

6.10 Evaluation of Off-Design Correlations . . . . . . . . . . . . 121

6.11 Variation of the Outlet Flow Angle with Incidence . . . . . . . . 122

7.1 Schematic of Wake Trajectories for Varying Flow Conditions . . 128

7.2 Test Matrix for PAKD-A and PAKD-F Airfoils . . . . . . . . . 130

7.3 Profile Losses for PAKD-A and PAKD-F . . . . . . . . . . . 132

7.4 Instantaneous Losses for PAKD-A . . . . . . . . . . . . . . . 134

7.5 Comparison of Profile Losses for all Airfoils . . . . . . . . . . . . 136

A.1 Surface Static Pressure Taps on PAKB $\ldots \ldots \ldots \ldots 3$

A.2 Surface Static Pressure Taps on PAKD-A . . . . . . . . . . . . . . 154

A.3 Surface Static Pressure Taps on PAKD-F . . . . . . . . . . . . . 155

B.1 PAKD-F: Loading Distribution at Design Incidence and FSTI = $1.5 \% \ldots \ldots \ldots \ldots \ldots$. . . . . . . . . . . . . . . . . . . .

B.2 PAKD-F: Loading Distributions at $\mathrm{Re}=\mathbf{8 0 , 0 0 0}$ and FSTI $=\mathbf{1 . 5 \%} 160$

B.3 PAKD-F: Zweifel Coefficient Variation with Incidence . . . . . . 161 
C.1 Instantaneous Losses for $\mathbf{f}=\mathbf{0 . 5} \ldots \ldots \ldots \ldots$. . . . . . . . 164

C.2 Instantaneous Losses for $\mathbf{f}=1.0 \ldots \ldots \ldots$. . . . . . . . 165

C.3 Instantaneous Losses for $\mathbf{f}=1.6 \ldots \ldots \ldots 6$

C.4 Instantaneous Losses for $\mathbf{f}=\mathbf{2 . 0} \ldots \ldots \ldots$. . . . . . . . 167

C.5 Instantaneous Losses for $\mathbf{f}=\mathbf{2 . 5} \ldots \ldots \ldots \ldots$

C.6 Instantaneous Losses for $\mathbf{f}=\mathbf{3 . 0} \ldots \ldots \ldots$. . . . . . . . . . 169 


\section{List of Symbols}

$\begin{array}{ll}\text { a } & \text { Constant } \\ \text { A } & \text { King's law constant } \\ \text { C } & \text { King's law constant } \\ C_{d} & \text { Blade chord length } \\ C_{P} & \text { Boundary layer dissipation coefficient } \\ C_{P b} & \text { Base pressure coefficient } \\ C_{P l} & \text { Blade static pressure coefficient } \\ C_{P 0} & \text { Total pressure coefficient } \\ C_{P S} & \text { Static pressure coefficient } \\ C_{Q} & \text { Dynamic pressure coefficient } \\ C_{Q W T} & \text { Wind tunnel calibration coefficient } \\ C_{V} & \text { Velocity coefficient } \\ \mathrm{d} & \text { Leading edge diameter } \\ \mathrm{E} & \text { Ancmometer, Transducer Voltage } \\ \mathrm{f} & \text { Reduced Frequency } \\ \mathrm{F} & \text { Force } \\ F_{Y} & \text { Tangential loading }\end{array}$

xii 


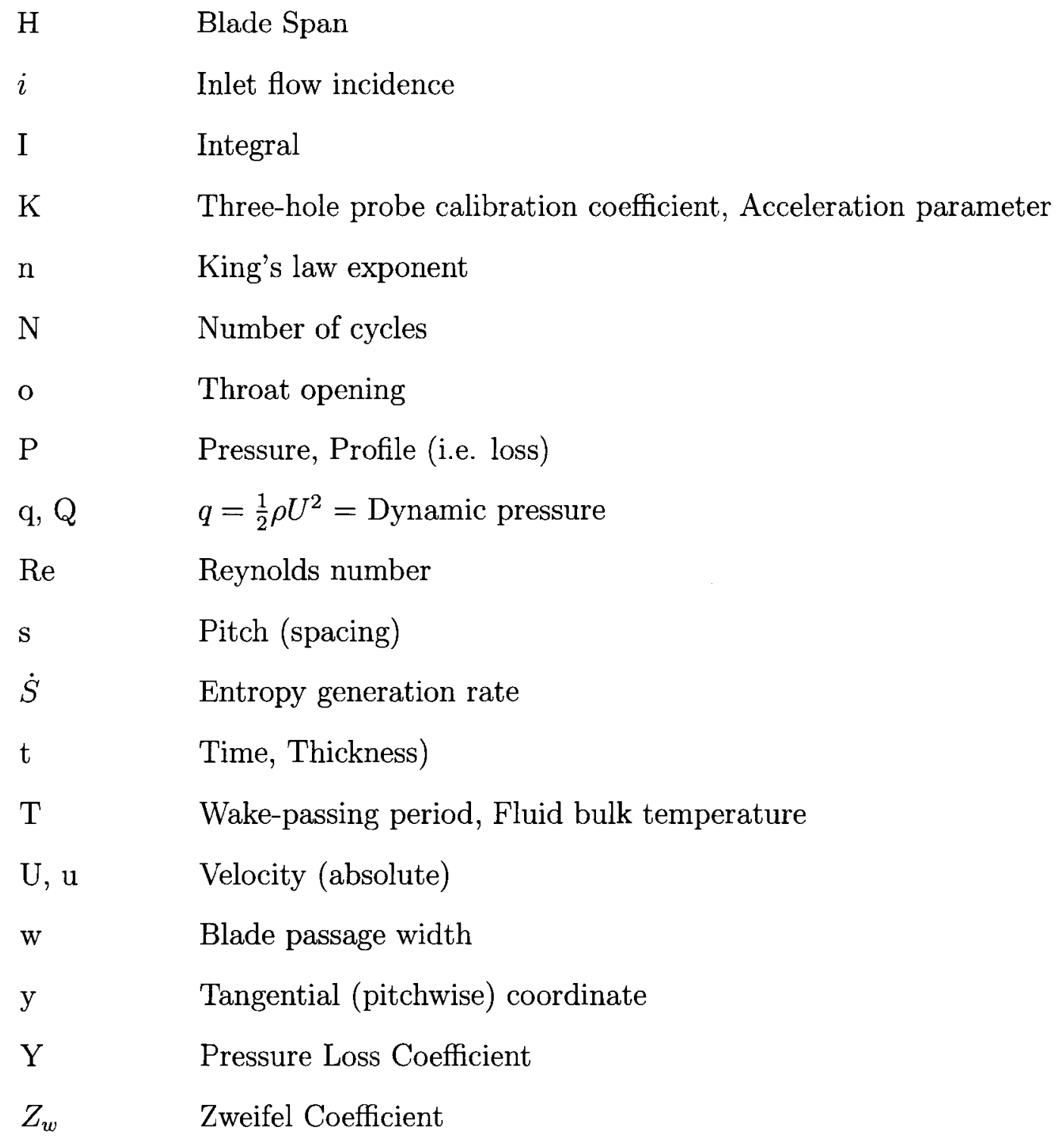

\section{Greek}

$\alpha$

Flow angle (measured from the axial direction)

$\beta \quad$ Metal angle (measured from the axial direction)

$\gamma \quad$ Stagger angle (measured from the axial direction)

$\delta \quad$ Boundary layer thickness

$\delta^{*} \quad=\int_{0}^{\delta}\left(1-\frac{U}{U_{b l}}\right) d z=$ Boundary layer displacement thickness

$\Delta \quad$ Delta (difference) 
$\theta \quad=\int_{0}^{\delta}\left(1-\frac{U}{U_{b l}}\right) \frac{U}{U_{b l}} d z=$ Boundary layer momentum thickness

$\Lambda \quad$ Integral length scale in streamwise direction

$\mu \quad$ Dynamic viscosity

$\nu \quad$ Kinematic viscosity

$\rho \quad$ Density

$\sigma \quad=\frac{C}{s}=$ Solidity

$\phi \quad$ Flow coefficient

$\varphi \quad$ Probe angle relative to the axial position

\section{Subscripts}

0

Total (stagnation) conditions

1

Inlet

2

Outlet

$\mathrm{b}$

Bar, Base

$\mathrm{C}$

Chord

des

Design

$\mathrm{e}$

Exit (Outlet)

i

Inlet

ideal

Ideal conditions

1

Local

$\mathrm{m}$

Mixed-out

$\max$

Maximum

$\mathrm{P}$

Profile

ref

Reference conditions

$\mathrm{S}$

Static conditions

tot

total (i.e. loss) 


$\begin{array}{ll}\mathrm{x} & \text { Axial } \\ \mathrm{y}, \mathrm{Y} & \text { Tangential (pitchwise) } \\ \mathrm{t} & \text { Time } \\ \mathrm{l} & \text { Local } \\ \delta & \text { Boundary layer thickness based }\end{array}$

\section{Superscripts}

, Mass-averaged in pitchwise direction

$\begin{array}{ll}\text { Abbreviations } \\ \text { AC } & \text { Alternating Current } \\ \text { AVR } & \text { Axial Velocity Ratio } \\ \text { FSTI } & \text { Free Stream Turbulence Intensity } \\ \text { HP } & \text { Horse Power } \\ \text { LE } & \text { Leading Eedge } \\ \text { LP } & \text { Low-Pressure } \\ \text { LPT } & \text { Low-Pressure Turbine } \\ \text { PS } & \text { Pressure Side } \\ \text { RMS } & \text { Root Mean Square } \\ \text { SS } & \text { Suction Side } \\ \text { SLF } & \text { Surface Length Fraction } \\ \text { SSL } & \text { Suction Surface Length } \\ \text { TE } & \text { Trailing Edge } \\ \text { TOB } & \text { Top Of the Bridge }\end{array}$




\section{Chapter 1}

\section{Introduction}

The low-pressure turbine (LPT) is located at the downstream end of a turbofan engine. In typical turbofan engines, the LPT is used to drive the fan and the lowpressure compressor. The LPT consists of a number of stages and may account for up to one third of the engine weight (Howell et al., 2001). Since today's LP turbines operate with an efficiency above $90 \%$, it is becoming progressively more difficult to make further improvements to the LP turbine efficiency (Hodson and Steele, 1994), thus a lot of effort at the present time is directed towards cost savings and improvement in engine economics through a reduction in the number of blades

A reduced number of blades means that each blade is subjected to higher aerodynamic loading. These blades are often referred to as the highly-loaded blades. This thesis will compare three blades belonging to the same family. The blades were designed to serve the same duty, sharing the same axial chord and the inlet and outlet design flow angles. The first blade, designated PAKB, is the baseline version of the family. It is the midspan profile of an LPT vane designed by Pratt \& Whitney 
Aircraft (PWA). The profile was inversely redesigned for low-speed testing. The other two blades, also designed by PWA and designated PAKD-A and PAKD-F, are designed for $25 \%$ higher loading. The primary goal of this study was to investigate the impact of increased loading on the airfoil aerodynamic performance. Additionally, the influence of loading distribution on performance was studied, since the main difference between two blades with higher loading is in the pressure distribution. The PAKD-A has a very similar (aft-loaded) pressure distribution to the baseline airfoil, PAKB, while PAKD-F is front-loaded.

The Reynolds numbers in LP turbines are lower than in the other engine components. Operation at low Reynolds numbers typically results in significant lengths of laminar flow on the low pressure (suction) side of the airfoil. This can result in flow separation on the aft-end of the airfoil where the pressure gradient is adverse. Flow separation is tolerated when the flow reattaches before the trailing edge forming a separation bubble, since it does not increase the losses significantly. However, if the flow fails to reattach, the blade stalls and a significant increase in losses occurs. Higher loading results in stronger adverse pressure gradients, and therefore highly-loaded turbine blades are more prone to flow separation. The previous studies that were part of the same project by Mahallati (2003) and Dai (2004) examined the Reynolds number sensitivity for PAKB and PAKD-A, respectively. Thus, the first phase of the present study focused mainly on PAKD-F. In this phase, the airfoil was exposed to a wide range of Reynolds numbers (25,000 to 150,000 based on inlet velocity and the axial chord) and two free stream turbulence intensities $(1.5 \%$ and $4 \%)$.

Aircraft engines often operate at off-design conditions during takeoff, idle, climb and cruise. As a result, the inlet flow angles and/or Reynolds number are different 
from their design values. The sensitivity to varying Reynolds number was the subject of the first phase of this study. The effects of changes in the inlet flow angle were the main focus of the second phase. All three airfoils were examined under incidence values of $-5^{\circ}$ and $+5^{\circ}$ relative to the design incidence and the same turbulence intensities as in the first phase. This part of the study was done at a constant Reynolds number of 80,000 .

In the third and final phase of the project, the effects of blade row interactions were studied. The flow through a blade row in the engine is affected by the wakes coming from an upstream blade row. This results in so-called periodically-unsteady flow. The blade performance under such conditions can be appreciably different from the performance under steady-flow conditions. The PAKB airfoil was extensively tested under unsteady conditions by Mahallati (2003). The current unsteady measurements focused on the two PAKD airfoils. The measurements were done at a Reynolds number of 50,000 and freestream turbulence intensity of $4 \%$. To simulate the effects of the wakes, a moving-bar wake-generator was used. The wake-generator consists of a rotating wheel with spokes (bars) mounted around its circumference. The spokes were designed to produce wakes with the same characteristics as a typical upstream airfoil.

Both steady and unsteady measurements were performed at midspan only. The midspan (profile) losses, outlet flow angles and the loading distributions were measured. The measurements were performed in a low-speed linear cascade using a three-hole pressure probe, static pressure taps on the airfoil surfaces and a singlewire hotwire probe.

Chapter 2 will provide an overview of the past profile loss investigations for lowpressure turbine airfoils. It will also introduce the terminology and nomenclature that 
will be used throughout this thesis. The experimental set-up, instrumentation and calibration will be described in Chapter 3, while the subsequent chapter will focus on the measurement procedures and data reduction. The steady flow measurements for Reynolds number sensitivity at design incidence will be presented and discussed in Chapter 5, while the results at off-design incidence will be the subject of Chapter 6. The unsteady loss measurements will be presented and discussed in Chapter 7 . Finally, conclusions and recommendations for the future research will be given in the last chapter. 


\section{Chapter 2}

\section{Literature Review}

\section{$2.1 \quad$ Introduction}

This chapter provides an overview of the previous studies most pertinent to the topics of the current investigation. An introduction to the terminology and nomenclature that will be used throughout the thesis will be provided in Section 2.2. Section 2.3 will discuss the Zweifel coefficient, which is used to compare the loading levels between different blades. The transition from laminar to turbulent flow plays an important role in the airfoil performance. A brief overview of transition modes will be provided in Section 2.4. The subsequent section will discuss the profile loss generation mechanisms. Section 2.6 will briefly discuss unsteady blade row interactions. Section 2.7 will summarize the previous investigations on both steady and unsteady flow in low-pressure (LP) turbines that are relevant to the current project. Finally, the loss correlations will be discussed in Section 2.8, since they were compared with the measured losses obtained in the present measurements. 


\subsection{Nomenclature and Terminology}

\subsubsection{Airfoil Nomenclature}

Figure 2.1 presents the airfoil nomenclature used throughout this thesis. All flow and metal angles are measured from the axial direction. It should be noted that for the purpose of the current study the incidence was defined as the difference between the design $\left(\alpha_{1 \text { des }}\right)$ and actual $\left(\alpha_{1}\right)$ inlet flow angles as given by Eq. 2.1.

$$
i=\alpha_{1}-\alpha_{1 d e s}
$$

Although the leading edge diameter $(d)$ and inlet wedge angle $\left(W_{e}\right)$ parameters were not employed in the PAKB and PAKD airfoil design, they are shown in Figure 2.1 because of their relevance to the discussion involving the evaluation of empirical loss prediction correlations in Section 2.8.

\subsubsection{Loss Breakdown Terminology}

A conventional loss breakdown divides the losses into following categories:

- Profile losses

- Secondary losses

- Tip-leakage losses

Profile losses are the subject of the thesis. They include both the losses generated by viscous effects in the blade surface boundary layers and the losses attributed to 


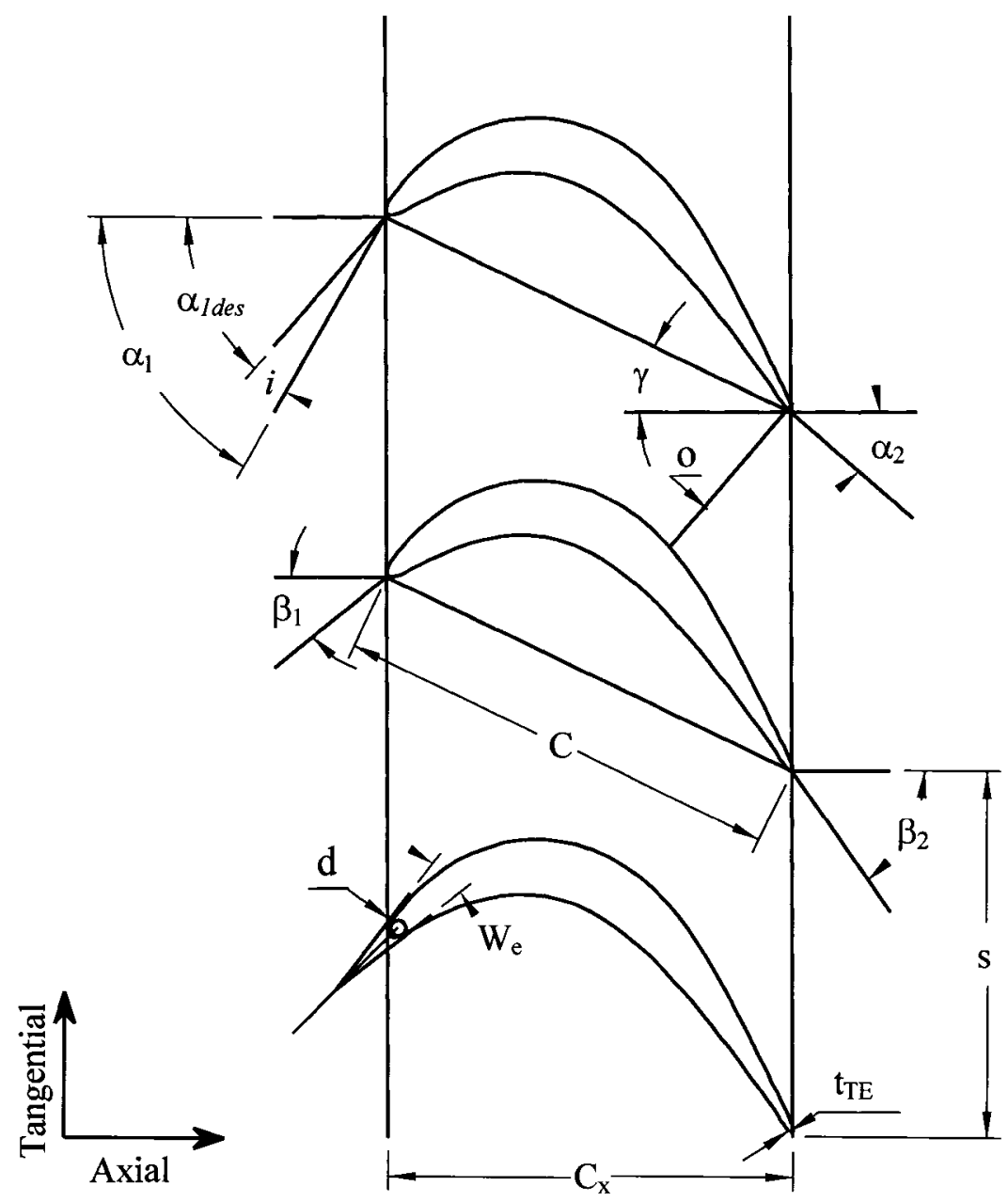

$\alpha_{1}$ : inlet flow angle

$C_{x}$ : axial chord

$\alpha_{1 \text { des }}$ : design inlet flow angle

$C$ : true chord

$\alpha_{2}$ : outlet flow angle

$i$ : incidence

$\beta_{1}$ : inlet metal angle

$s$ : pitch (spacing)

$\beta_{2}$ : outlet metal angle

$\gamma$ : stagger angle

$W_{e}$ : inlet wedge angle

$d$ : leading edge diameter

$o$ : throat opening

$t_{T E}$ : trailing edge thickness

Figure 2.1: Terminology of Linear Cascade Airfoils 
the trailing edge due to the pressure acting on it. In LP turbines, the profile losses are often predominant (Howell et al., 2001). The profile losses in turbines are usually expressed through total pressure loss coefficient, $Y_{P}$ :

$$
Y_{P}=\frac{P_{01}^{\prime}-P_{02}^{\prime}}{q_{2}^{\prime}}
$$

where $P_{0}^{\prime}$ and $q^{\prime}$ are the mass-averaged total and dynamic pressures at the mid-span, respectively, and 1 and 2 correspond to inlet and outlet planes. A further discussion of profile losses will be provided in Section 2.5.

Secondary losses are the result of complex flows in the endwall regions. Although they are usually lower than the profile losses in the low-pressure turbines, the

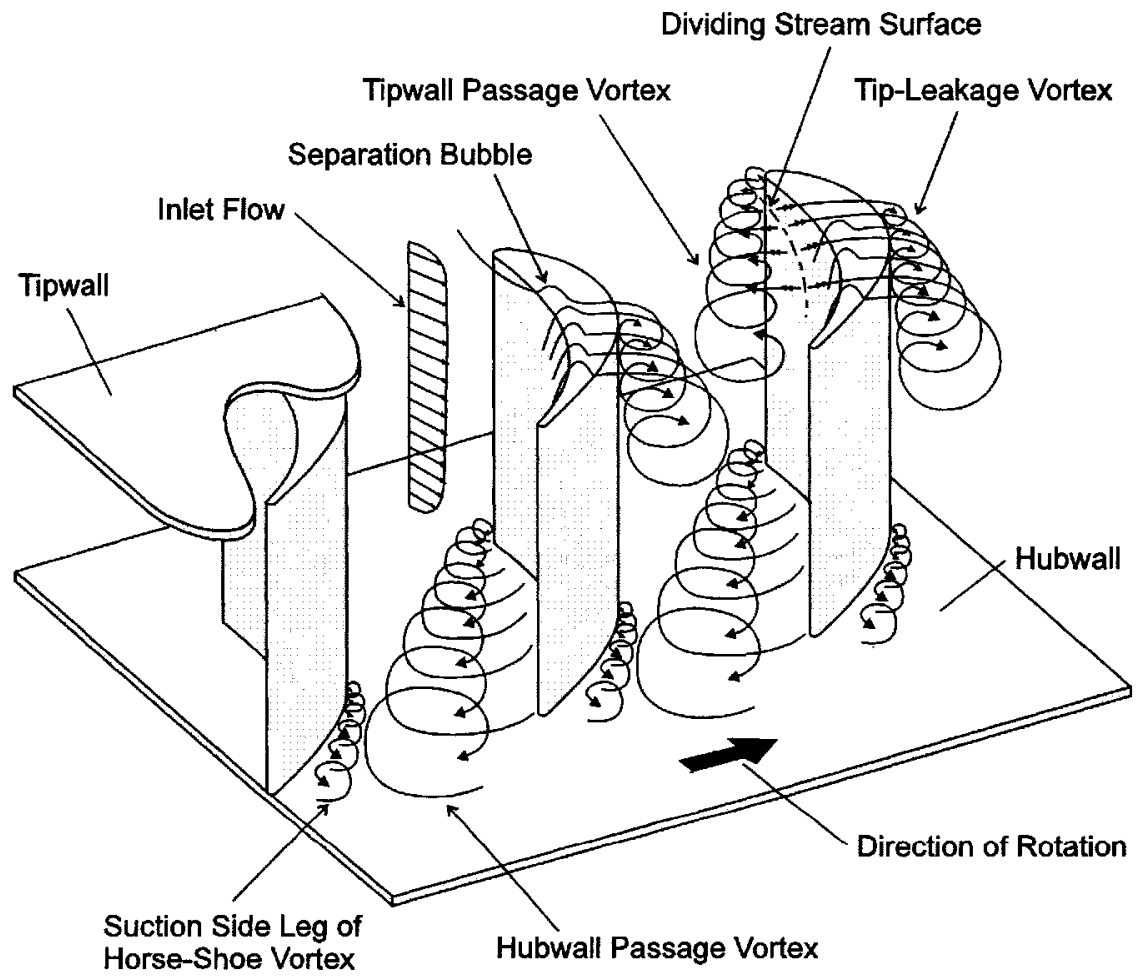

Figure 2.2: Schematic of the Flow through a Turbine Blade Passage 
secondary losses are not negligible. A concurrent project by Zorić (2006) is focused on the secondary losses that occur in the PAKB and PAKD blade passages. Tip-leakage losses, found near the tip of the rotor, are associated with the leakage flow over the tip of the rotor blades. Both the secondary and tip leakage flows involve the formation of streamwise vortices and the mixing out of these vortices is a major source of the corresponding losses. A schematic view of the blade passage flow with major features is shown in Figure 2.2.

\subsection{Zweifel Coefficient}

Zweifel (1945) introduced a coefficient defined as the ratio of the actual $\left(F_{Y}\right)$ to the ideal tangential force $\left(F_{Y \text { ideal }}\right)$ :

$$
Z_{w}=\frac{F_{Y}}{F_{Y \text { ideal }}}=2\left(\frac{s}{C_{X}}\right) \cos ^{2}\left(\alpha_{2}\right)\left(\tan \left(\alpha_{2}\right)-\tan \left(\alpha_{1}\right)\right)
$$

The ideal tangential force, not physically attainable, represents the tangential force that can be achieved, while avoiding adverse pressure gradients on the surfaces of the airfoil. Such ideal loading would be obtained through a pressure distribution with the inlet total pressure acting on the pressure side and the outlet static pressure on the suction side of the airfoil.

Based on available data from the 1930s and 1940s, Zweifel (1945) concluded that $Z_{w}$ of 0.8 gave minimum profile losses. Values of $Z_{w}$ lower than this resulted in higher losses because of increased wetted area due to high solidity $(C / s)$. On the other hand, values of $Z_{w}$ in excess of 0.8 , resulted in high profile losses due to separation (because of lower solidity). Blade design technology improved considerably since the 1940s 
and values of $Z_{w}$ well in excess of 1.0 are common nowadays. The motivation for continuous increase in the blade loading and $Z_{w}$ with corresponding reduction in blade count comes from desire to decrease the engine weight, as discussed in Chapter 1. The airfoils that will be examined in the present thesis, PAKB and PAKDs, have the Zweifel coefficients of 1.08 and 1.36 , respectively.

\subsection{Modes of Transition and Laminar Separation Bubbles}

\subsubsection{Modes of Transition}

In general, there are three modes of transition from laminar to turbulent flow boundary layers: natural, bypass and separated-flow transition. In addition, it is also possible to have a reverse transition from turbulent to laminar flow. This mode is also known as "relaminarization", and can occur in very strongly accelerated turbulent flows. This section gives a brief overview of the transition modes, mainly based on Mayle (1991).

Natural transition is common in laminar boundary layers under mild pressure gradients and for freestream turbulence intensity (FSTI) of less than about $0.1 \%$. The process consists of several stages, as shown in Figure 2.3. The transition begins when a critical value of the boundary layer thickness is reached. The laminar boundary layer becomes susceptible to small disturbances and two-dimensional (Tollmien-Schlichting) waves develop. As they move downstream, the waves are amplified and transformed into unstable three-dimensional waves. The process continues with the roll-up of the waves into loop vortices, which break down forming the first turbulent spots. The 


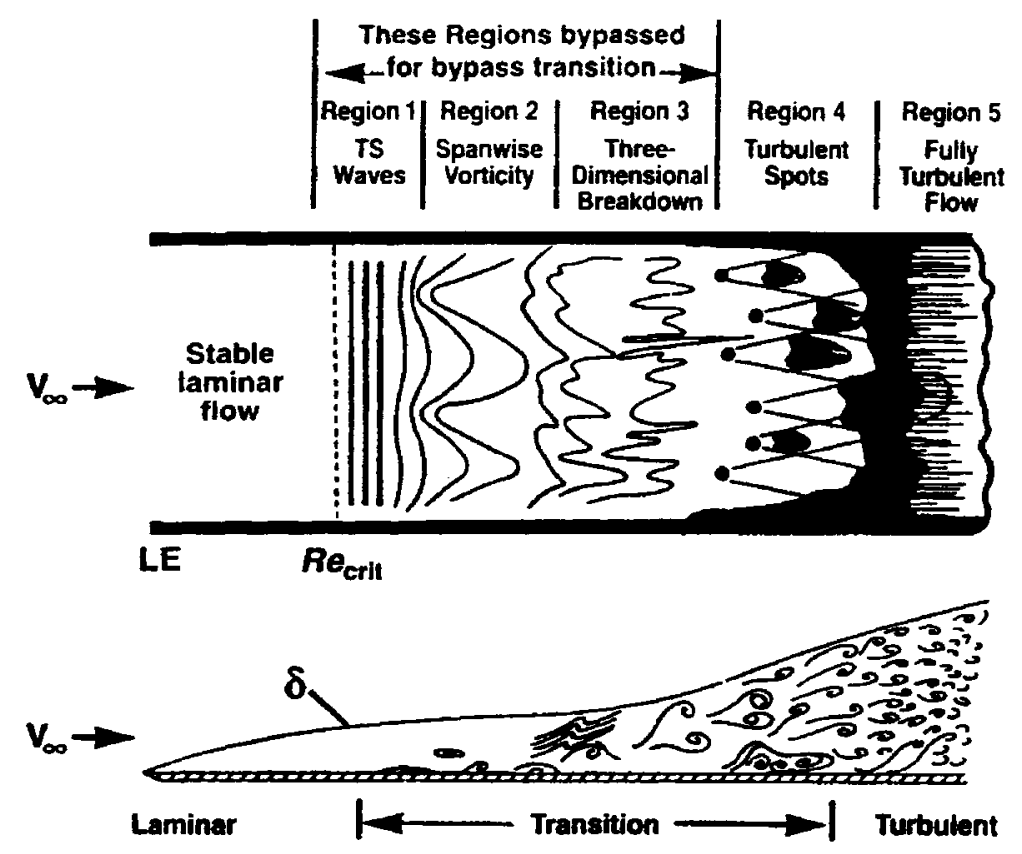

Figure 2.3: Schematic View of Attached Flow Transition Process (reproduced from Schlichting and Gersten, 2000)

spots then grow both in longitudinal and lateral directions until they eventually form a turbulent boundary layer. Since typical values of FSTI in turbomachinery are much higher than $0.1 \%$, natural transition does not normally occur in these machines.

When the flow is subjected to high FSTI, generation of turbulent spots occurs directly in the boundary layer and it becomes susceptible to disturbances. Thus, the initial stages of the natural transition process are bypassed, and this transition mode is also known as bypass transition. Mayle (1991) indicated that bypass transition is the most common transition mode in turbomachinery. When compared to the natural mode, the transition length of the bypass mode is shorter. This is due to the fact that in the bypass mode, regions 1 through 3 shown in Figure 2.3 are absent. 
In regions with strong adverse pressure gradients at low Reynolds numbers, it is not uncommon to have laminar separation bubbles present on the blade suction surface. Once the flow separates from the surface, the resulting free shear layer will have an inflection point in its velocity distribution, and this makes it receptive to disturbances. Therefore, a transition and subsequent reattachment of the flow may occur. This is the third mode of transition mentioned earlier, and is a common one in low-pressure turbines. Separation bubbles can affect the airfoil performance significantly; therefore, a more detailed description of separation bubbles will be given in the next section.

So far the discussion has dealt with transition from laminar to turbulent flow. Transition from turbulent to laminar flow is also possible and, as mentioned, this is called reverse transition or relaminarization. The details of the actual process are still not fully understood, but it is believed that when the turbulent flow undergoes strong acceleration, the turbulence is dissipated through viscous effects resulting in the relaminarized boundary layer. The acceleration parameter, $\mathrm{K}$, is a measure of acceleration and thus the susceptibleness of the flow to relaminarization. $\mathrm{K}$ is defined as:

$$
K=\frac{\nu}{U_{b l}^{2}}\left(\frac{d U_{b l}}{d x}\right)
$$

where $U_{b l}$ is the velocity at the edge of the boundary layer and $\nu$ is kinematic viscosity of the fluid. It is commonly accepted that when the value of $\mathrm{K}$ is greater than $3 \cdot 10^{6}$, the reverse transition is expected to occur (Mayle, 1991). It is thought that the freestream turbulence levels do not play an important role in this process, and that when the acceleration is strong enough the relaminarization will occur regardless of the turbulence intensity in the freestream flow. 


\subsubsection{Laminar Separation Bubbles}

A schematic view of a separation bubble and its major features is shown in Figure 2.4. The separation bubble is formed when a laminar boundary layer detaches from the surface, usually due to adverse pressure gradient. Downstream of the separation point, a free shear layer is formed. The free shear layer cannot support streamwise pressure gradients and in most situations the pressure gradient on the surface beneath the separated flow is reduced to approximately zero. For this reason separation bubbles are normally identified by a plateau in the surface pressure distribution. The free shear layer is very receptive to the instabilities, such as Kelvin-Helmholtz, causing the shear layer to roll-up into vortices. As these instabilities amplify, the transition process begins. At the upstream end of the separated region, the flow trapped below the separated shear layer is essentially stagnant. However, downstream of the transition onset point, a reverse flow vortex is formed. It is believed that the transition is complete at the point of maximum bubble displacement. The turbulent mixing between the separated shear layer and adjacent freestream flow reduces the displacement effect of the bubble until the flow eventually reattaches. When the flow does not reattach, it is said that the blade is stalled.

Separation bubbles have been traditionally classified as "short" or "long" (Gaster, 1967). The classification is not based on their length, but on their influence on the overall pressure distribution. Short bubbles have only local displacement effect on the pressure distribution, while the long bubbles appreciably affect the overall pressure distribution as shown in Figure 2.5. The process of changing the bubble state from short to long is referred to as "bursting" (Mayle, 1991). "Bursting" results in a noticeable change in the tangential force and may lead to stall. This topic will be further discussed in Chapter 5. 


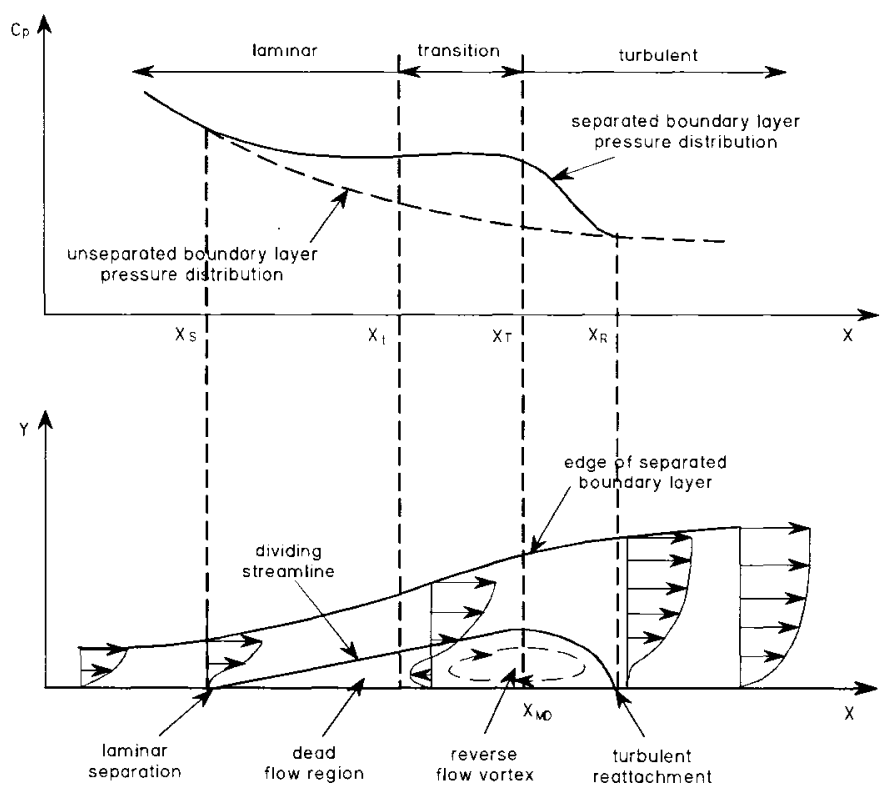

Figure 2.4: Schematic View of Separation Bubble (reproduced from Zhang, 2002)

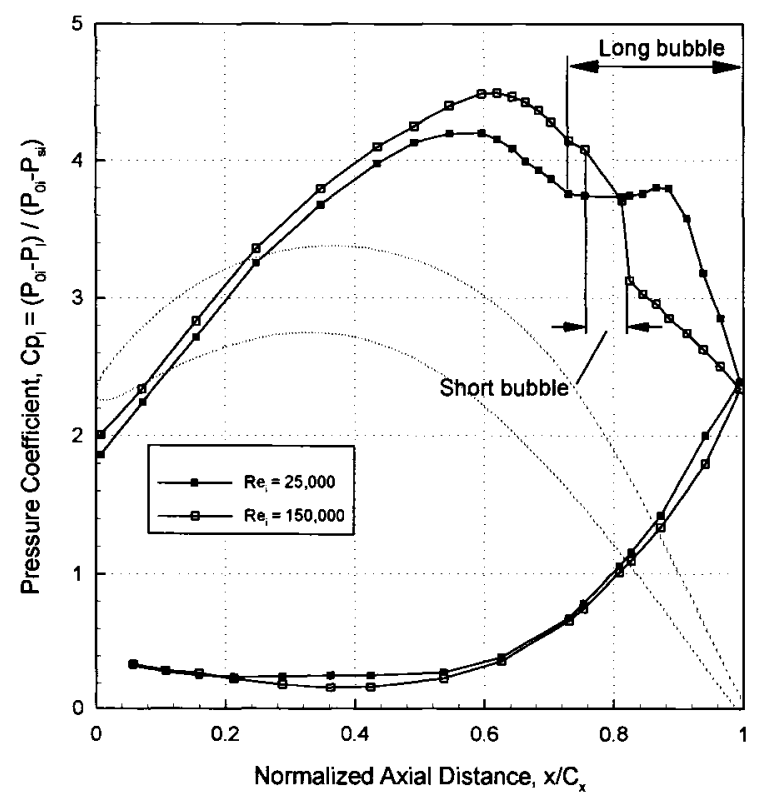

Figure 2.5: Short vs. Long Separation Bubbles

(based on data from Mahallati, 2003) 


\subsection{Profile Loss Generation Mechanisms}

This section will discuss the profile loss generation. A typical variation of the losses with Reynolds number will also be presented to illustrate the importance of boundary layer transition on the loss production.

As previously mentioned, the profile loss $\left(Y_{P}\right)$ combines both the losses generated in the boundary layers on the blade surface and the loss arising from the finite thickness of the trailing edge and the pressure acting on it. Denton (1993) gives the following relation for profile losses:

$$
Y_{P}=-\frac{C_{p b} t_{T E}}{w}+\frac{2 \theta}{w}+\left(\frac{\delta^{*}+t_{T E}}{w}\right)^{2}
$$

where: $w$ is the passage width $(w=s \cos (\gamma))$ and $t_{T E}$ is the trailing edge thickness. $C_{p b}$ is the base pressure coefficient is defined as and $\left.C_{p b}=\left(P_{b}-P_{2}^{\prime}\right) / q_{2}^{\prime}\right)\left(P_{b}\right.$ is the static pressure at the trailing edge). The first term on the right hand side of the equation is the loss due to low base pressure, which acts as a retarding force on the trailing edge. The second term is loss generated in boundary layers on the blade surfaces. The boundary layer parameters $\theta$ and $\delta^{*}$ are the values just before the trailing edge. This term is a direct measure of the loss production in the boundary layers, since the value of $\theta$ includes the mixing at the edge of any separation bubble that might be present on the blade surface. The final term accounts for the combined blockage of the trailing edge and the boundary layers. Typically the loss production mainly occurs in the boundary layers (particularly the suction side boundary layer), and it can be related to the entropy production per unit width $(\dot{\mathrm{S}})$, defined in Eq. 2.6 . 


$$
\dot{S}=\int_{L E}^{T E} \frac{C_{d} U_{b l}^{3} \rho}{T} d x
$$

where $C_{d}$ is the dimensionless dissipation coefficient, $U_{b l}$ is the boundary layer edge velocity, $\rho$ is the fluid density and $T$ is the fluid's bulk temperature. LE and TE refer to the leading and trailing edges, respectively. $C_{d}$ cannot be calculated without knowing the details of the state of the boundary layer. Variations of $C_{d}$ with the momentum thickness Reynolds number $\left(R e_{\theta}\right)$ in zero pressure gradient for both laminar and turbulent boundary layers are shown in Figure 2.6. The dissipation is slightly increased for the accelerating boundary layer, and reduced in one near the separation point (Denton, 1993).

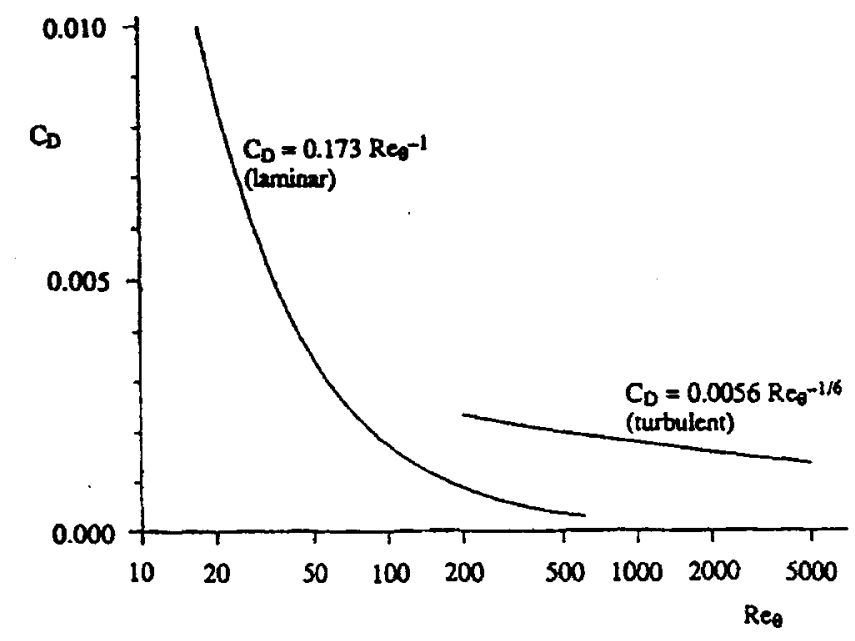

Figure 2.6: Dissipation Coefficient for Laminar and Turbulent Boundary Layers (reproduced from Denton, 1993)

The dissipation coefficient $\left(C_{d}\right)$ is more dependent on $R e_{\theta}$ for laminar than for turbulent boundary layers. It is also worth noting that for the range of $R e_{\theta}$ where either laminar or turbulent boundary layer can exist (approximately for $200<R e_{\theta}<$ 800 ), it is better to have a laminar boundary layer than a turbulent one as this will 
result in lower losses. The dissipation coefficient for laminar boundary layers in this region is less than that for turbulent boundary layers by a factor of between 2 and 5 . On the other hand, laminar boundary layers are much more prone to separation than turbulent ones. A turbine designer desires to maximize the extent of the laminar flow as long as that does not result in a massive separation and stall. This highlights the importance of predicting the location of transition for an effective airfoil design.

In addition to the dissipation coefficient, the entropy generation in the boundary layers is strongly influenced by the velocity on the edge of the boundary layer $\left(U_{b l}\right)$. As shown in the Eq. 2.6, the entropy generation varies as $U_{b l}{ }^{3}$. Figure 2.7 shows a typical velocity distribution on the surface of a highly loaded blade. It can be clearly seen that much higher velocities are encountered on the suction side (upper curve) of the airfoil. This together with the possible presence of a separation bubble results in the boundary layer on the suction side playing a more important role in the loss generation than the boundary layer on the pressure side of the airfoil.

Section 2.4 discussed the boundary layer transition and laminar separation bubbles. So far in this section, the issue of loss generation in the boundary layers was addressed as well as the factors affecting it. To illustrate the effects of transition and separation bubble on airfoil performance, a typical variation of the profile losses with the Reynolds number is shown in Figure 2.8. The transition at high Reynolds numbers normally occurs via bypass mode ahead of the location where the laminar boundary layer would have separated. In these situations, the flow is fully attached over the suction surface as illustrated on the right hand side of Figure 2.8. When the transition occurs via bypass mode, the variation of losses with Reynolds number is complex and depends on details of the velocity distribution and the relative proportions of the surface that is covered by laminar or turbulent boundary layer. The net result is 


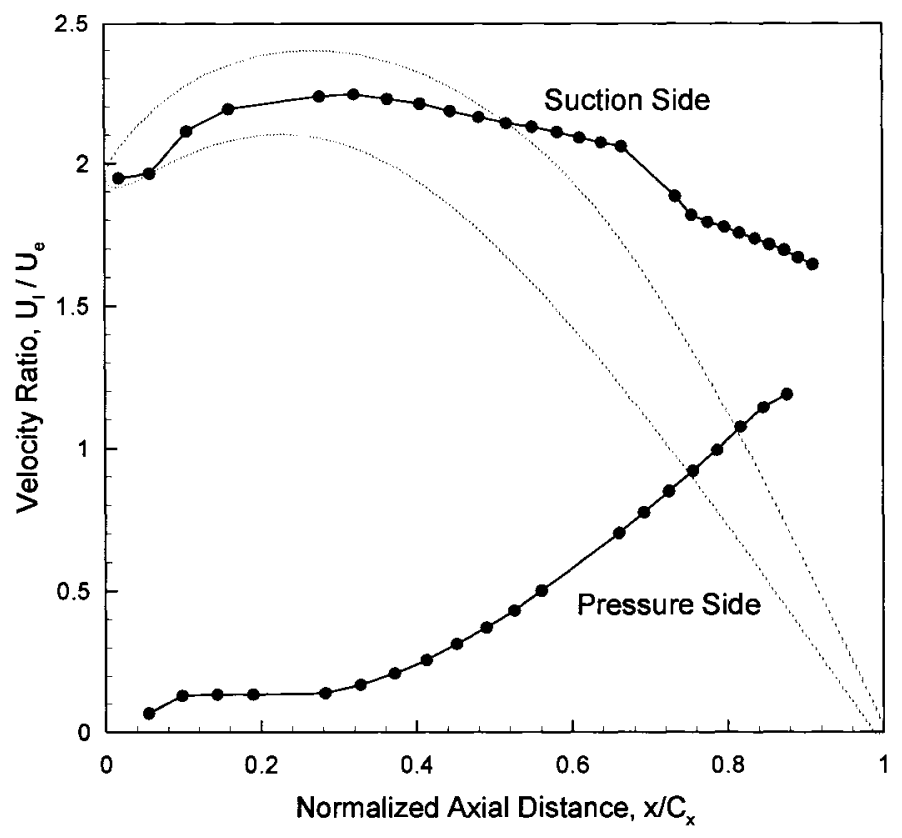

Figure 2.7: Velocity Distribution

influenced by two separate and counteracting factors according to Denton (1993). With a decrease in Reynolds number:

- the transition moves downstream, decreasing the wetted area of the blade covered by the turbulent flow. Since the losses generated in the laminar boundary layer are less than those generated in the turbulent one for the same range of $R e_{\theta}$, the losses decrease.

- the dissipation within the laminar boundary layer is increased due to the lower $R e_{0}$, as shown in Figure 2.6. This factor contributes to the increase in the loss production.

With further reduction in Reynolds number, laminar separation takes place before the transition, and a laminar separation bubble is formed increasing the profile losses 


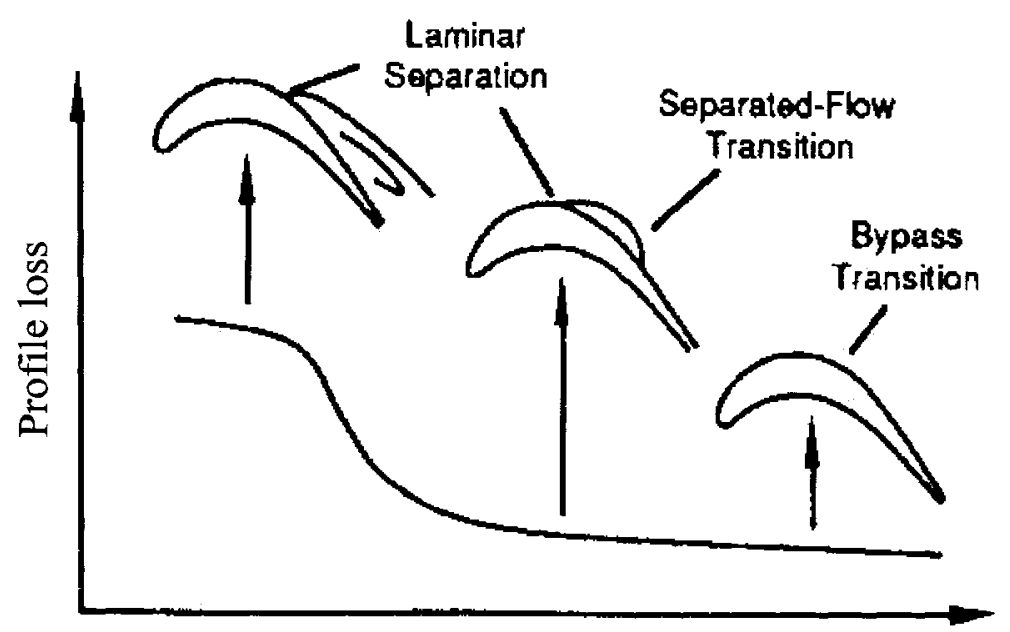

Reynolds number $\ln (\mathrm{Re})$

Figure 2.8: Profile Losses and Transition for a Various Reynolds numbers (reproduced from Mayle, 1991)

(middle drawing, Figure 2.8). At low Reynolds numbers $\left(R e_{c}<10^{5}\right)$, the losses increase rapidly due to the high dissipation in the laminar boundary layer and the growth of the separation bubble. As the Reynolds number is reduced even further, the bubble continues to grow until it fails to reattach and the blade is stalled (left drawing, Figure 2.8). This stalling results in a steep loss increase.

\subsection{Overview of Unsteady Flow and its Influence on Losses}

Traditional airfoil design is based on the simplifying assumption that the flow entering each row is steady, or in other words, there is no blade row interaction. Steady cascade measurements provide a great deal of details of the losses, the loadings, 
and the boundary layer development. However, they are not truly representative of the real engine environment. To keep the turbine compact, the axial gap between adjacent rows in a typical engine is commonly of the order of $1 / 2$ of the axial chord or less. Thus, the flow does not mix-out completely before entering the next blade row, resulting in flow unsteadiness.

A typical stator-rotor interaction as seen from the stationary frame of reference is shown in Figure 2.9. The wakes leaving the stator blades are chopped into segments by the rotor blades. The velocity varies greatly within the blade passage with higher velocities near the suction surface and lower near the pressure surface of the blades. This contributes to the wake distortion that is evident in Figure 2.9.

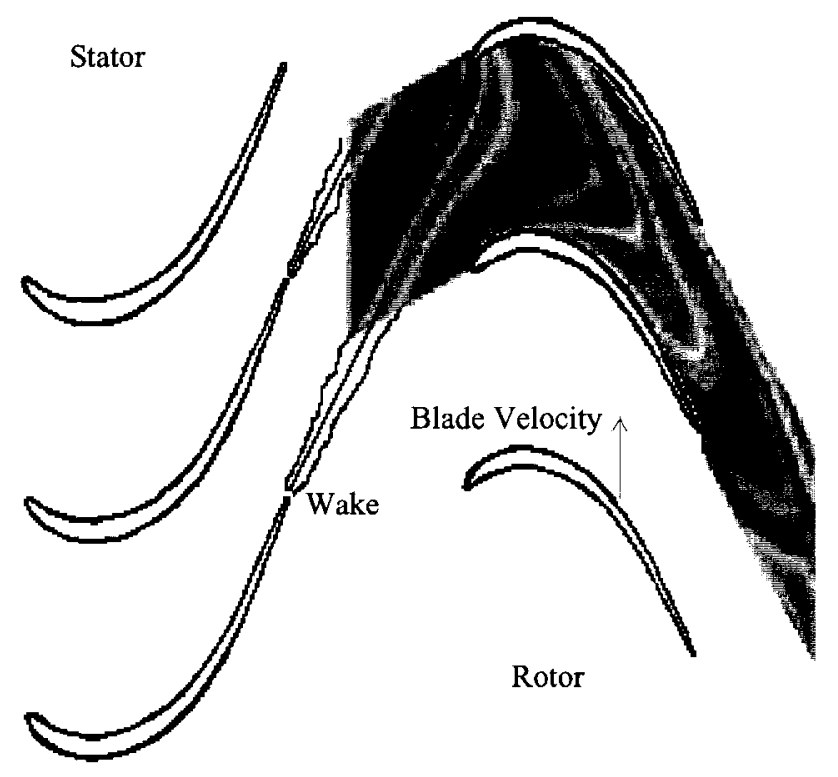

Figure 2.9: Schematics of Stator-Rotor Interaction (reproduced from Hodson and Dawes, 1998) 
As they are convected through the passage, the wakes interact with the boundary layers. The result of that interaction depends, among other things, on whether the boundary layer is laminar of turbulent. A number of studies, some of them summarized in in the next section, have been performed to examine the details of the role of the wake induced transition when the surface layer is laminar. Based on the previous studies, several conclusions can be drawn.

First, if a laminar separation bubble is present either on the suction or the pressure side of an airfoil, wake passing promotes transition and flow reattachment, thus shortening the bubble. Second, when a laminar boundary layer is exposed to the mild adverse pressure gradient, the wake passing promotes an earlier transition often via the bypass mode, and thus increases the wetted area covered by the turbulent boundary layer. On the other hand, it is believed that when the laminar boundary layer is subjected to the strong acceleration the disturbances from the wake may not result in earlier transition in the attached boundary layer.

The effects of the flow unsteadiness on the losses depend on the frequency of the wake passing. For example, if the frequency is low then there is enough time between the wakes for a separation bubble to reestablish. On the other hand, if the frequency is high and the successive wakes are closely spaced the bubble may be suppressed completely. It should be also noted that for high frequency, the close spacing between the wakes can cause a larger fraction of the surface to have turbulent flow, which would be expected to cause an increase in losses.

A typical variation of losses with the wake passing frequency, or "reduced frequency", as it will be defined in Chapter 7, is shown in Figures 2.10 and 2.11. At low Reynolds number, when a large bubble or a massive separation is present on the suction side of the blade under steady flow, introduction of periodically-unsteady 
wakes results in reduction of the bubble size, and consequently the losses. However, at higher Reynolds numbers, when the bubble is small under steady conditions, the figures show that the periodic wake passing in fact increases the overall loss levels. Both Schulte and Hodson (1998) and Mahallati (2003) concluded that there is an optimum reduced frequency and wake strength that results in the minimum profile losses. As mentioned earlier, Mahallati's measurements were made for the PAKB airfoil. One of the goals of the present work is to examine the corresponding behavior of the more highly-loaded PAKD airfoils when they are subjected to unsteady wake passing.

\subsection{Summary of Experimental Investigations}

This section presents a tabular summary of the most relevant experimental investigations related to the present work. Both steady and periodically-unsteady flow conditions are included. The abbreviations used in Table 2.1 are given below:

Type of Experiment:

FP Flat Plate

LC Linear Cascade

RR Rotating Rig

Type of Measurements:

PP Pressure Probe

HW Hotwire Probe

HF Hotfilm Array

OV Oil Visualization

SP Surface Pressure Taps 


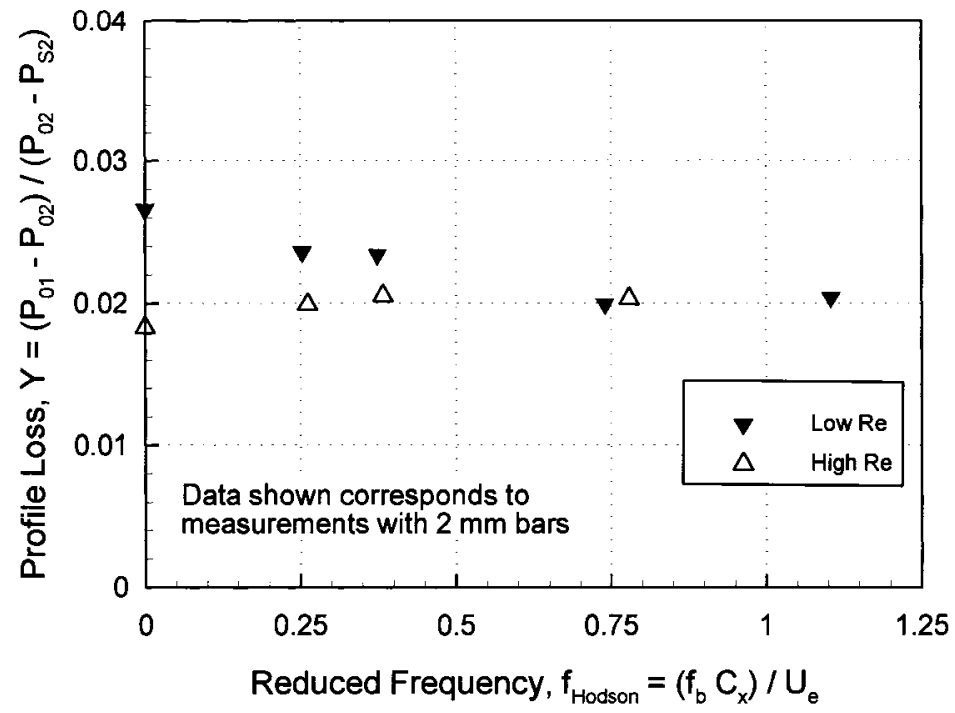

Figure 2.10: Profile Loss Variation under Unsteady Conditions (reproduced based on data from Schulte and Hodson, 1998)

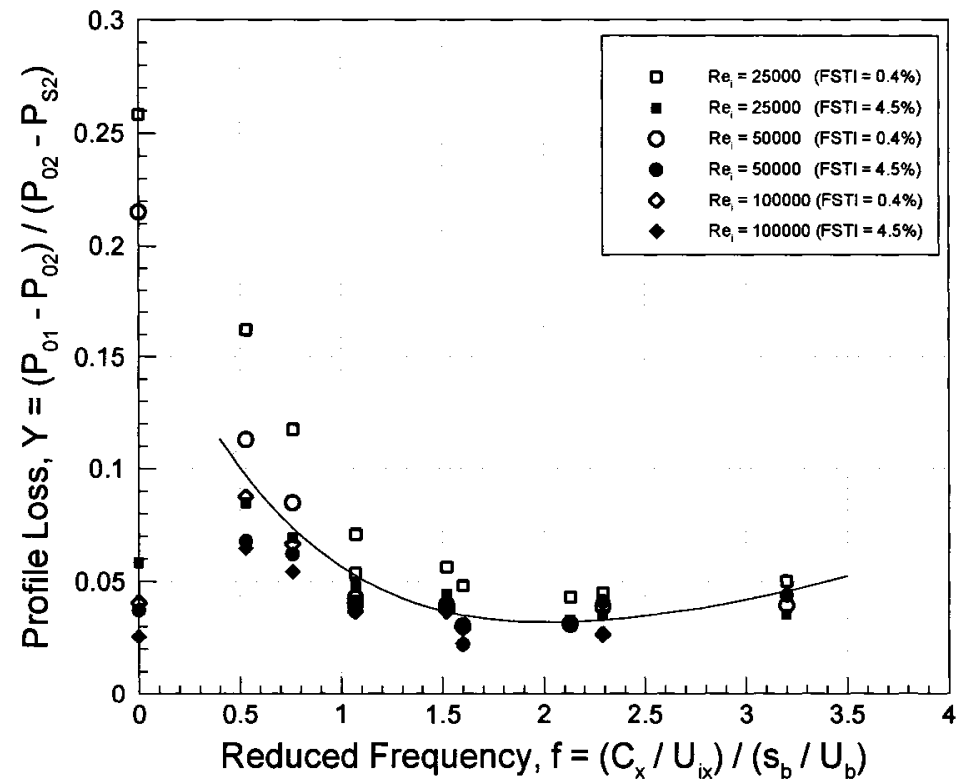

Figure 2.11: Profile Loss Variation under Unsteady Conditions for PAKB (reproduced from Mahallati, 2003) 
SV Smoke Visualization

\section{Parameters Investigated:}

i Incidence (not defined for RR experiments)

f Reduced Frequency

LD Loading Distribution

M Mach number

Re Reynolds number $\cdot 10^{3}$ based on:

1 - axial chord and inlet velocity

2 - true chord and exit velocity

3 - suction surface length and exit velocity

4 - suction surface length and inlet velocity

$\mathrm{Tu}$ Freestream Turbulence Intensity (FSTI)

s/C Pitch-to-Chord ratio

$\phi \quad$ Flow Coefficient 


\begin{tabular}{|c|c|c|c|c|c|c|c|c|c|c|c|c|}
\hline \multirow[b]{2}{*}{ Reterence } & \multicolumn{4}{|c|}{ Exporiment and Meassurement Details } & \multicolumn{3}{|c|}{ Parameters } & \multicolumn{4}{|c|}{ Experimentalal Conditions } & \multirow[b]{2}{*}{ Comments } \\
\hline & Type & Probe & $v_{i z}$ & Looding & & 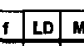 & 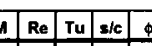 & ildeg] & $\mathbf{R e}_{\mathbf{e}}$ & Tu[\%] & Unsteady & \\
\hline Amrtit (1993) & RR & $\mathrm{HF}$ & & & & & $x$ & N/A & $120,170,220^{2}$ & & & 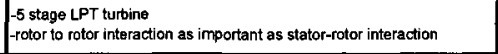 \\
\hline Benner (2003) & 10 & pp & $0 y$ & $x$ & & & & 0.0070 & $300^{1}$ & 04 & & 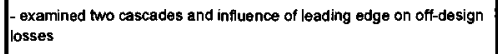 \\
\hline Brear et al (2002) & LC & PP, HW & sv & $x$ & & & & $-10,0,10$ & $100-300^{2}$ & $0.6-4$ & YES & 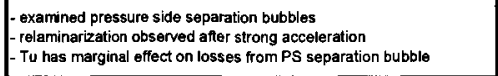 \\
\hline Brear and Hodson (2004) & Lc & HW & sv & & & & & $-10,0,10$ & $130^{2}$ & 0.6 & YES & 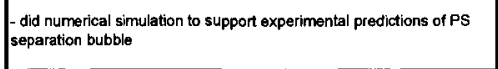 \\
\hline Dai (2004) & $\llcorner\mathrm{LC}$ & $P p$ & & $x$ & & & & 0 & $35-150^{1}$ & $1.5,4$ & & 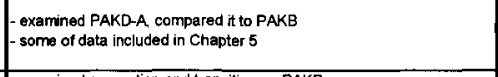 \\
\hline Domey et al (2000) & Lc & PP, HW & & $x$ & & & & 0 & 43-172' & 1 & & 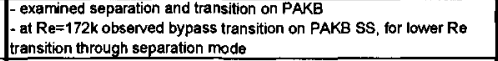 \\
\hline Gonzalez et al. (2001) & Lc & Pp & & $x$ & & & $x$ & 0 & $80-320^{2}$ & 0.5 & YES & 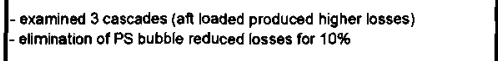 \\
\hline Goobie ef al (1989) & $L C$ & PP & ov & & & & & $-15,0,15$ & $100-300^{1}$ & 1.5 & & 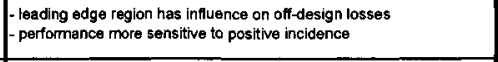 \\
\hline Hodson and Dominy (1987) & Lc & pp & ov & $x$ & & & & $9,0,20$ & $30-200^{2}$ & & & -tests in transonic wind tunnel \\
\hline Hodson el al (1994) & $R R$ & HF & & & & & & N/A & $90 \cdot 180^{2}$ & & YES & 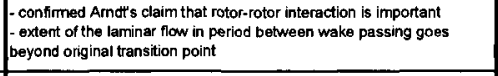 \\
\hline Hoheisel et al. (1987) & Lc & Pp & & & & & & -37.7 to 12.3 & $100-1100^{2}$ & $0.8-7.1$ & & 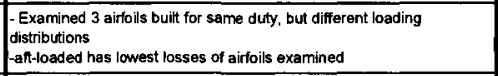 \\
\hline Hollon and Jacos (2001) & LC & $\mathrm{HF}$ & sv & & & & $x$ & 0 & $13.70^{4}$ & very low & & 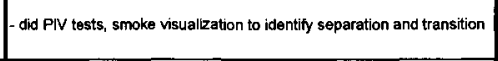 \\
\hline Houtemanans et al. (2004) & LC & $P P, H W$ & & $x$ & & & $x$ & $-9,0,3$ & $50 \cdot 200^{2}$ & 0.6 & & 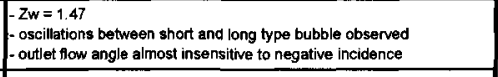 \\
\hline Howell ot al (2001) & LC & PP, HW, HF & & & & & & 0 & $100-210^{2}$ & 0.5 & YES & -aft-Laded distribution was not good tor high loading lovels \\
\hline Lake et al (1999) & $L C$ & PP, HW & & & & & $\times 1$ & 0 & 50 to $200^{1}$ & 1.4 & & -PAKB seperation and transtion \\
\hline Lou and Houmouziadis \&z & FP & HW, HF & & & & & 1 & 0 & $100-2000^{1}$ & 0.20 & YES & $\begin{array}{l}\text {-fat plate } \\
\text { - sporaration bubble invesitigates }\end{array}$ \\
\hline
\end{tabular}




\subsection{Empirical Profile Loss Prediction Methods}

In gas path design, mean line analysis and empirical loss correlations still continue to play an important role. One of the first and probably most widely used loss systems for axial turbines was developed by Ainley and Mathieson (1951) (A-M). They proposed the following correlation for profile losses:

$$
\left.Y_{P, A M}=\left(Y_{P\left(\beta_{1}=0\right)}+\left|\frac{\beta_{1}}{\alpha_{2}}\right|\left(\frac{\beta_{1}}{\alpha_{2}}\right)\left[Y_{P\left(\beta_{1}=\alpha_{2}\right)}-Y_{P\left(\beta_{1}=0\right)}\right)\right]\right)\left(\frac{t_{\max } / C}{0.2}\right)^{\frac{\beta_{1}}{\alpha_{2}}}
$$

where $t_{m a x} / C$ is the maximum thickness-to-chord ratio. $Y_{P\left(\beta_{1}=0\right)}$ and $Y_{P\left(\beta_{1}=\alpha_{2}\right)}$ are the loss coefficients for the nozzle and impulse blades shown in Figues 2.12 and 2.13, respectively. The nozzle and impulse blades represent the two extreme cases for a given outlet flow angle, with the former resulting in the maximum acceleration and the latter resulting in the zero net acceleration of the flow between inlet and outlet of the passage. For an arbitrary blade, the losses are taken as a weighted average of the two extremes.

Due to the continual improvements in design, changes in design philosophies and accumulation of test cases, there is a need for periodic updates to the correlations. A number of updates of A-M have been published. The first major update was due to Dunham and Came (1970). They included an improved Reynolds number correction as follows:

$$
f(R e)= \begin{cases}\left(\frac{R e_{c}}{2 \cdot 10^{5}}\right)^{-0.2} & \text { for } R e_{c} \leq 2 \cdot 10^{5} \\ 1 & \text { for } 2 \cdot 10^{5}<R e_{c} \leq 10^{6} \\ \left(\frac{R e_{c}}{10^{6}}\right)^{-0.2} & \text { for } R e_{c}>10^{6}\end{cases}
$$

where $R e_{c}$ is Reynolds number based on the outlet velocity and true chord. This correction is applied to the profile loss calculated from Eq. 2.7: 


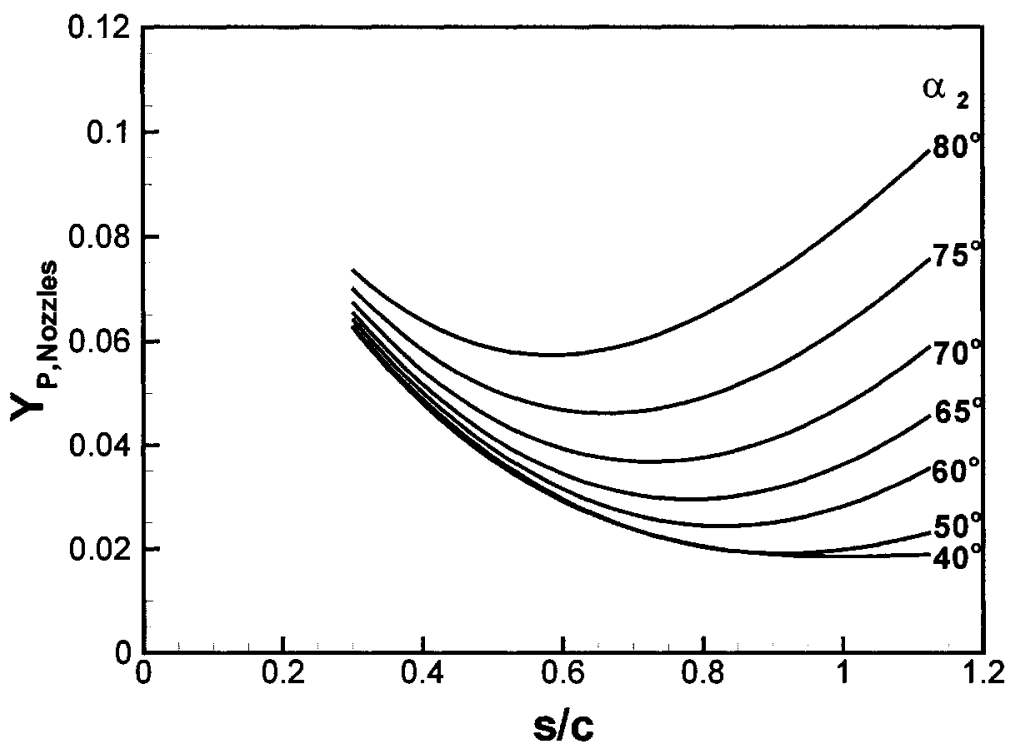

Figure 2.12: A-M Loss Coefficients for Nozzle Blades

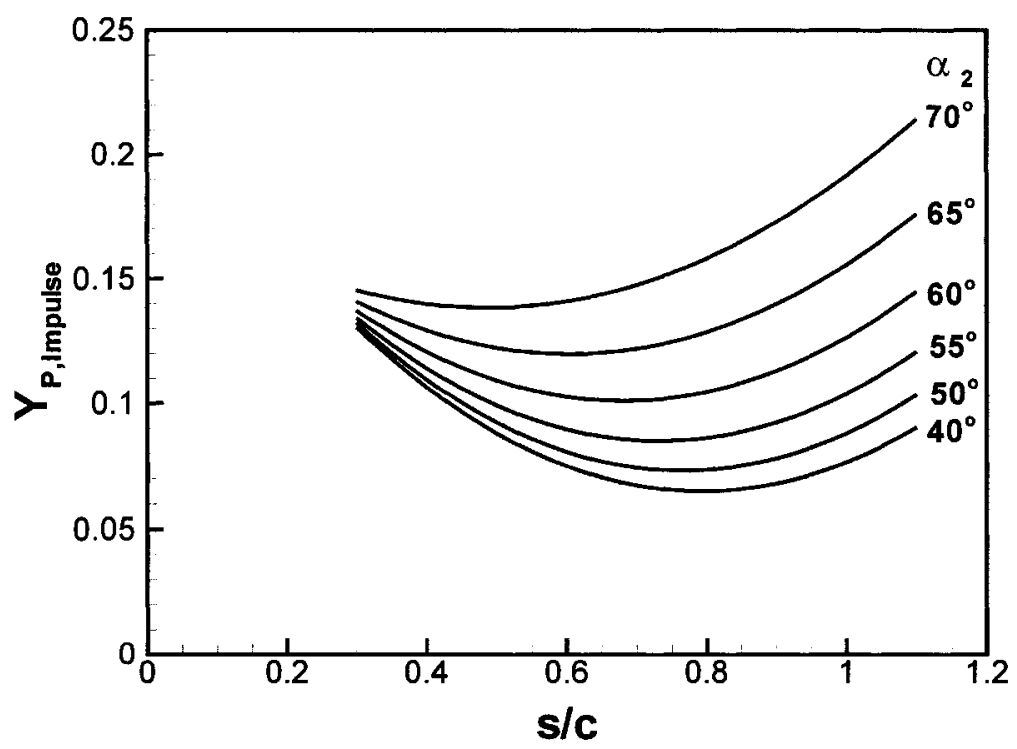

Figure 2.13: A-M Loss Coefficients for Impulse Blades 


$$
Y_{P, A M D C}=Y_{P, A M} f(R e)
$$

The form of correlation given in Equations from 2.7 to 2.9 is normally referred to as the Ainley/Mathieson/Dunham/Came correlation (AMDC).

The remainder of this section will focus on the correlations which will be further discussed and evaluated in the thesis, namely those due to: Kacker and Okapuu (1982) and Zhu and Sjolander (2005) for design incidence, and Moustapha et al. (1990) and Benner et al. (1997) for off-design incidence loss predictions.

The modifications to AMDC by Kacker and Okapuu (K-O) separated the trailing edge loss from the profile loss, since it was difficult to justify predicting trailing edge loss simply through a multiplier on the profile loss as was done by AMDC. This results in an additional correction (0.914) to the surface losses and the addition of a separate trailing edge loss coefficient, $Y_{T E T}$, as given in Eq. 2.11. Secondly, K-O proposed that a factor of $2 / 3$ should be applied to the AMDC profile losses. This factor took into account advances in the aerodynamic design since Ainley and Mathieson's time. At low Reynolds numbers, airfoils exhibit higher loss levels, as discussed in Section 2.5. To improve the prediction of the loss variation for low Reynolds numbers, K-O proposed a stronger low Reynolds number correction, which took the following form:

$$
f(R e)=\left\{\begin{array}{cl}
\left(\frac{R e_{c}}{2 \cdot 10^{5}}\right)^{-0.4} & \text { for } R e_{c} \leq 2 \cdot 10^{5} \\
1 & \text { for } 2 \cdot 10^{5}<R e_{c} \leq 10^{6} \\
\left(\frac{R e_{c}}{10^{6}}\right)^{-0.2} & \text { for } R e_{c}>10^{6}
\end{array}\right.
$$


The final K-O correlation for the profile losses then takes the form:

$$
Y_{P}=0.914\left(2 / 3 Y_{P, A M} k_{p}+Y_{\text {shock }}\right) f(R e)+Y_{T E T}
$$

where $Y_{P, A M}$ is the profile loss as per the A-M correlation and $Y_{T E T}$ is the loss associated with the trailing edge and is a function of the ratio of trailing-edgethickness-to-throat-opening $\left(t_{T E} / o\right) . \quad Y_{\text {shock }}$ is the loss associated with shocks and equals 0.0 for incompressible flow, as is the case in low-speed wind tunnel testing. Finally, $k_{p}$ is the correction factor taking into account channel flow acceleration, and is equal to 1.0 for incompressible conditions.

The other improvements introduced by Kacker-Okapuu dealt with the secondary flows and compressibility effects which are not the scope of this thesis, and therefore will not be discussed.

The K-O correlation showed significant discrepancies with recent experimental data. This led to a review of the correlation by Zhu and Sjolander (2005) (ZS), including comparison with recent results obtained for the present highly-loaded blades. A major shortcoming of the K-O correlation was again found at low Reynolds numbers. Although the K-O correlation included a stronger correction for Reynolds number effects than AMDC, it still underestimated the losses for Reynolds numbers lower than $2 \cdot 10^{5}$. In addition to proposing an improved Reynolds number correction, Z-S also modified the influence of the maximum thickness-to-chord ratio $\left(t_{\max } / C\right)$. Finally, K-O incorporated a factor of $2 / 3$ to account for profile design improvements in the period between A-M and K-O. Based on recent data, Zhu and Sjolander concluded that the factor of $2 / 3$ may be too optimistic for nozzle blades with axial inflow $\left(\beta_{1}=0\right)$. Therefore, the factor was modified accordingly. The final Z-S correlation 
takes the following form:

$$
\left.Y_{P}^{\prime}=\left(2 / 3 Y_{P\left(\beta_{1}=0\right)}+\left|\frac{\beta_{1}}{\alpha_{2}}\right|\left(\frac{\beta_{1}}{\alpha_{2}}\right)\left[2 / 3 Y_{P\left(\beta_{1}=\alpha_{2}\right)}-0.825 Y_{P\left(\beta_{1}=0\right)}\right)\right]\right)\left(\frac{t_{m a x} / C}{0.2}\right)^{K_{m} \frac{\beta_{1}}{\alpha_{2}}}
$$

where $K_{m}$ is defined as follows:

$$
\begin{array}{cc}
K_{m}=-1 & \text { for } t_{\text {max }} / C>0.2 \\
K_{m}=1 & \text { for } t_{\text {max }} / C \leq 0.2
\end{array}
$$

The profile loss due to Z-S is then given as:

$$
Y_{P}=0.914\left(Y_{P}^{\prime} k_{p}+Y_{\text {shock }}\right) f(R e)+Y_{T E T}
$$

and the low Reynolds number correction is modified as follows:

$$
f(R e)=\left(\frac{R e_{c}}{2 \cdot 10^{5}}\right)^{-0.575} \text { for } \operatorname{Re}_{c} \leq 2 \cdot 10^{5}
$$

The original A-M loss system provided profile loss predictions for both design incidence (as given here) and off-design incidence, while both the $\mathrm{K}-\mathrm{O}$ and Z-S correlations are intended for use at design incidence only. They require companion correlations for off-design incidence, as described below.

Moustapha et al. (1990) proposed a new correlation for off-design profile losses for use with the design incidence correlation of Kacker-Okapuu. Unlike the earlier A-M off-design correlation, the new correlation accounts for the influence of the leading edge diameter, $d$, on the losses. Moustapha et al. correlated the additional losses at off-design incidence with the following incidence parameter. 


$$
X^{\prime}=\left(\frac{d}{s}\right)^{-1.6}\left(\frac{\beta_{1}}{\beta_{2}}\right)^{-2}\left[\alpha_{1}-\alpha_{1 d e s}\right]
$$

where $d / s$ is the leading edge diameter normalized by the blade pitch. Rather than using profile loss coefficient $\left(Y_{P}\right)$, the losses were given in the form of the kinetic energy coefficient $\left(\phi_{P}^{2}\right)$. The energy loss coefficient can be converted to the equivalent total pressure coefficient using:

$$
\Delta Y_{P}=\frac{1}{1-\Delta \phi_{P}^{2}}-1
$$

which is then added to the profile loss coefficient as given by the design point correlations ( $\mathrm{K}-\mathrm{O}$ in the case of Moustapha et al.).

The kinetic energy coefficient is then given by:

$$
\Delta \phi_{P}^{2}=f\left(X^{\prime}\right)
$$

where $f\left(X^{\prime}\right)$ is:

$$
f\left(X^{\prime}\right)=0.778 \cdot 10^{-5} X^{\prime}+0.56 \cdot 10^{-7} X^{\prime 2}+0.4 \cdot 10^{-10} X^{\prime 3}+2.054 \cdot 10^{-19} X^{\prime 6}
$$

for $800>X^{\prime}>0$, while for $0>X^{\prime}>-800$ :

$$
f\left(X^{\prime}\right)=-5.1734 \cdot 10^{-6} X^{\prime}+7.6902 \cdot 10^{-9} X^{\prime 2}
$$

In 1997, Benner et al. updated the correlation of Moustapha et al's. correlation and included an additional correlating parameter, the inlet wedge angle $\left(W_{e}\right)$. This was an attempt to capture the effects on the losses of the curvature discontinuity that 
occurs at the blend point near the leading edge when a leading edge circle is used. Benner et al. argued that the wedge angle is a convenient but approximate measure of this discontinuity in curvature. They also found that best correlation was obtained with a value of the exponent on $d / s$ of -0.05 , as opposed to the Moustapha et al's value of -1.6. A lower value of an exponent on a parameter means that the correlation is less sensitive to changes in that parameter. Benner et al. proposed the following modified incidence parameter to replace Moustapha et al's $X^{\prime}$ as given Eq. 2.14.

$$
X=\left(\frac{d}{s}\right)^{-0.05} W_{e}^{-0.2}\left(\frac{\beta_{1}}{\beta_{2}}\right)^{-1.4}\left[\alpha_{1}-\alpha_{1 d e s}\right]
$$

Similar to the form used by Moustapha et al. in Eq. 2.16, the kinetic energy coefficient in this case is fitted to a polynomial.

$$
\Delta \phi_{P}^{2}=\sum_{i=1}^{8} a_{i} X^{i}
$$

where the constants, $a_{i}$, are as follows:

$$
\begin{array}{ll}
a_{1}=-6.149 \cdot 10^{-5} & a_{2}=+1.327 \cdot 10^{-3} \\
a_{3}=-2.506 \cdot 10^{-4} & a_{4}=-1.542 \cdot 10^{-4} \\
a_{5}=+9.017 \cdot 10^{-5} & a_{6}=+1.106 \cdot 10^{-5} \\
a_{7}=-5.318 \cdot 10^{-6} & a_{8}=+3.711 \cdot 10^{-7}
\end{array}
$$

for $X>0$, while for $X<0$ :

$$
\Delta \phi_{P}^{2}=1.358 \cdot 10^{-4} X^{2}-8.720 \cdot 10^{-4} X
$$

It should be emphasized that both off-design correlations are intended for use with traditionally designed airfoils, employing circular leading edges and the inlet wedge 
angle. As will be seen in Chapter 6, this results not only in difficulties when applying them to airfoils of more recent design that do not use circles to define the leading-edge geometry, but also in poor predictions of profile losses at off-design incidence.

This concludes the review of literature, the next chapter will discuss the experimental set-up. 


\section{Chapter 3}

\section{Experimental Apparatus}

\subsection{Introduction}

The present research was conducted in the Low-Speed Turbomachinery Laboratory (LSTL) at Carleton University. All experimental work was performed in the open-circuit wind tunnel using three different linear cascades: PAKB, PAKD-A, and PAKD-F. This chapter will provide descriptions of the facility, experimental apparatus and calibration procedures.

The first section will focus on the wind tunnel, test section, turbulence generating grids and the linear cascades. Section 3.3 provides a description of instrumentation and the probes used for the measurements. The Data Acquisition System will be briefly described in Section 3.4, while the calibration equipment and methods are described in the last section. 


\subsection{Low-Speed Wind Tunnel}

\subsubsection{Wind Tunnel}

The open-circuit wind tunnel commissioned by Rodger (1992) and shown in Figure 3.1 was used as the wind source. The air is drawn into the wind tunnel through a
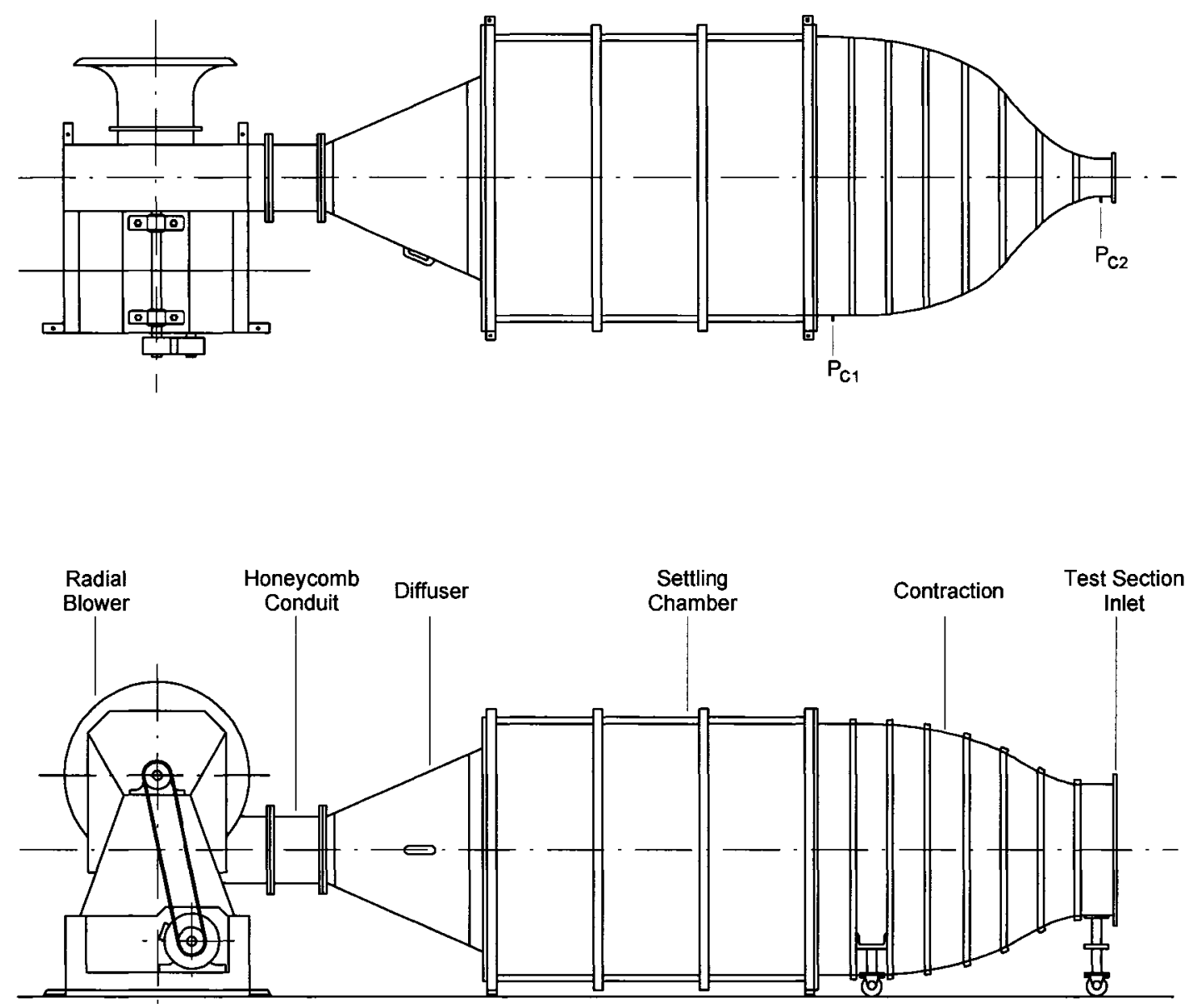

Figure 3.1: Schematic of Low-Speed Open-Circuit Wind Tunnel

(reproduced from Mahallati, 2003) 
bellmouth inlet by the radial blower which is connected to a 4-pole AC motor rated at 40 HP. The motor RPM can be varied using a solid-state frequency controller. The maximum airflow through the wind tunnel is about $5 \mathrm{~kg} / \mathrm{s}$ with a total pressure rise of about $1.2 \mathrm{kPa}$. A honeycomb with $45 \mathrm{~cm}$ deep cells is mounted at the outlet of the blower in order to remove the swirl present in the flow. The flow is diffused through a wide-angle rectangular diffuser equipped with five evenly spaced screens and then discharged to a settling chamber where four stainless screens are mounted in series. The air is then accelerated through a square-to-rectangular contraction with an area ratio of 14:1 before entering the test section. During the tests stable velocities ranging from $5 \mathrm{~m} / \mathrm{s}$ to $30 \mathrm{~m} / \mathrm{s}$ were attained at the test section inlet. Two static pressure taps, $\mathrm{P}_{C 1}$ and $\mathrm{P}_{C 2}$, are located as shown in Figure 3.1. They are used to set the operating point and provide reference pressures for pressure transducers, as will be discussed in Sections 4.2.1 and 4.2.2, respectively.

\subsubsection{Test Section}

Variable-incidence test section, designed by Goobie (1989) was used in the current experimental work. The flow enters the test section through a rectangular inlet 67.3 $\mathrm{cm}$ high and $20.3 \mathrm{~cm}$ wide. As shown in Figure 3.2, the test section is equipped with seven control surfaces that are used to set the inlet flow uniformity and outlet flow periodicity. In addition, the test section was modified by Mahallati (2003) to include the blockage mechanism marked as 1 in Figure 3.2. The mechanism improves the inlet flow uniformity in the upper portion of the cascade. More details on the procedure of setting the inlet flow uniformity and outlet flow periodicity will be provided in Section 4.2.3.

The linear cascade is mounted on a turntable, which can be rotated to allow inlet 


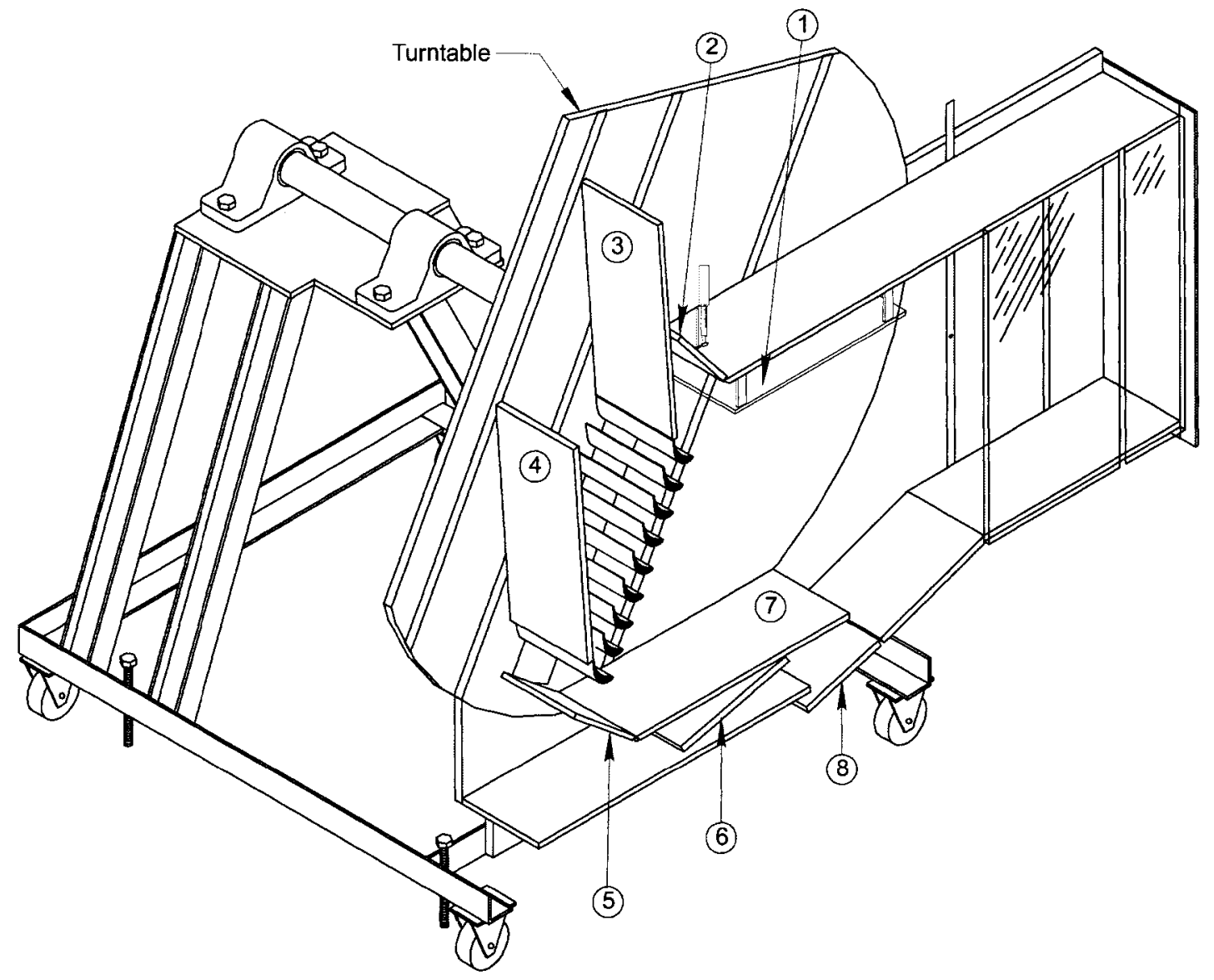
(1) Blockage Mechanism
(2) Top Flap
(3) Top Tailboard
(4) Bottom Tailboard

(5) Bottom Flap

(6) Upper By-pass

(7) Floating Wall

(8) Lower By-pass

\section{Figure 3.2: Isometric View of Test Section}

(reproduced from Mahallati, 2003)

flow incidence variation over a range of about $60^{\circ}$. A plexiglass window (not shown in Figure 3.2) represents the second endwall. It allows visual inspection of the cascade during the tests. For the unsteady part of the experiment, the plexiglass window was 
replaced with a wake-generator that will be described in Section 3.2.5.

On the backwall of the test section, a traverse mechanism can be mounted which allows two-axis linear motion of probes. A 4-phase stepper motor attached to a threaded rod drives each axis. The minimum step size for the traverse gear is 0.00635 $\mathrm{mm}$.

\subsubsection{Test Cascades}

During the present research, three different high-lift low-pressure (LP) turbine blades were studied. All three blades have the same inlet and outlet design flow angles and hence result in the flow having the same design turning angle of $95^{\circ}$. To fit into the same test section, the span of $203.2 \mathrm{~mm}$ ( $8 \mathrm{in}$ ) was kept the same for all three cascades.

Table 3.1: Summary of Airfoil Geometry

\begin{tabular}{|l|c|c|c|}
\hline & PAKB & PAKD-A & PAKD-F \\
\hline Axial Chord Length $\left(\mathbf{C}_{\mathbf{x}}\right)$ & $75.4 \mathrm{~mm}$ & $76.2 \mathrm{~mm}$ & $76.2 \mathrm{~mm}$ \\
\hline True Chord Length $(\mathbf{C})$ & $83.9 \mathrm{~mm}$ & $84.8 \mathrm{~mm}$ & $93.5 \mathrm{~mm}$ \\
\hline Blade Span $(\mathbf{H})$ & $203.2 \mathrm{~mm}$ & $203.2 \mathrm{~mm}$ & $203.2 \mathrm{~mm}$ \\
\hline Maximum Thickness $\left(\mathbf{t}_{\text {max }}\right)$ & $10.7 \mathrm{~mm}$ & $16.4 \mathrm{~mm}$ & $9.7 \mathrm{~mm}$ \\
\hline Trailing Edge Thickness $\left(\mathbf{t}_{\mathbf{T E}}\right)$ & $1.1 \mathrm{~mm}$ & $1.1 \mathrm{~mm}$ & $1.1 \mathrm{~mm}$ \\
\hline Stagger Angle $(\gamma)$ & $26.0^{\circ}$ & $26.0^{\circ}$ & $35.4^{\circ}$ \\
\hline Design Inlet Flow Angle $\left(\alpha_{\text {ides }}\right)$ & $35.0^{\circ}$ & $35.0^{\circ}$ & $35.0^{\circ}$ \\
\hline Design Outlet Flow Angle $\left(\alpha_{\text {edes }}\right)$ & $60.0^{\circ}$ & $60.0^{\circ}$ & $60.0^{\circ}$ \\
\hline Blade Spacing $(\mathbf{s})$ & $66.8 \mathrm{~mm}$ & $84.2 \mathrm{~mm}$ & $84.2 \mathrm{~mm}$ \\
\hline Number of Airfoils in Cascade & 9 & 7 & 7 \\
\hline Aspect Ratio $(\mathbf{H} / \mathbf{C})$ & 2.42 & 2.40 & 2.17 \\
\hline Solidity $(\sigma)$ & 1.26 & 1.01 & 1.11 \\
\hline Zweifel coefficient $\left(\mathbf{Z}_{\mathbf{w}}\right)$ & 1.08 & 1.36 & 1.36 \\
\hline
\end{tabular}


The first blade, PAKB, is the baseline version of this blade family, and it was first tested in the LSTL by Mahallati (2003). This turbine blade represents a midspan section of an LP turbine vane designed by Pratt and Whitney Aircraft (PWA). The profile was inversely redesigned for low-speed testing. The main difference between PAKB and the two PAKD airfoils is in the pitch, which was, for the PAKD's increased by approximately $25 \%$, resulting in fewer blades. Therefore, each blade is subjected to higher loading. The blade loading is given by the Zweifel coefficient $\left(\mathrm{Z}_{w}\right)$, defined in Section 2.3 (the summary of airfoil geometry parameters and Zweifel coefficients are given in Table 3.1). PAKB has $Z_{w}$ of 1.08, while the two PAKD airfoils have $Z_{w}$ of 1.36. Although the two PAKD airfoils share the same Zweifel coefficient, their loading distributions were intentionally made different in order to study effects of loading distribution on airfoil performance. This was achieved mainly by changing the stagger angle and the maximum thickness of the blades, as can be seen in Figure 3.3. The influence of loading distribution will be addressed in Chapters 5 through 7 .

\subsubsection{Turbulence Generating Grids}

The transition process from laminar to turbulent flow is greatly affected by the freestream turbulence intensity (FSTI) as discussed in Section 2.4. FSTI is a measure of fluctuations in the flow velocity and is defined as:

$$
F S T I=\frac{u^{\prime}}{U} \cdot 100 \%
$$

where $U$ is the mean velocity of the turbulent flow and $u^{\prime}$ represents the root mean square of the instantaneous fluctuations in the flow direction. The integral length scale $(\Lambda)$ is used to specify the size of the typical eddy in a turbulent flow. 


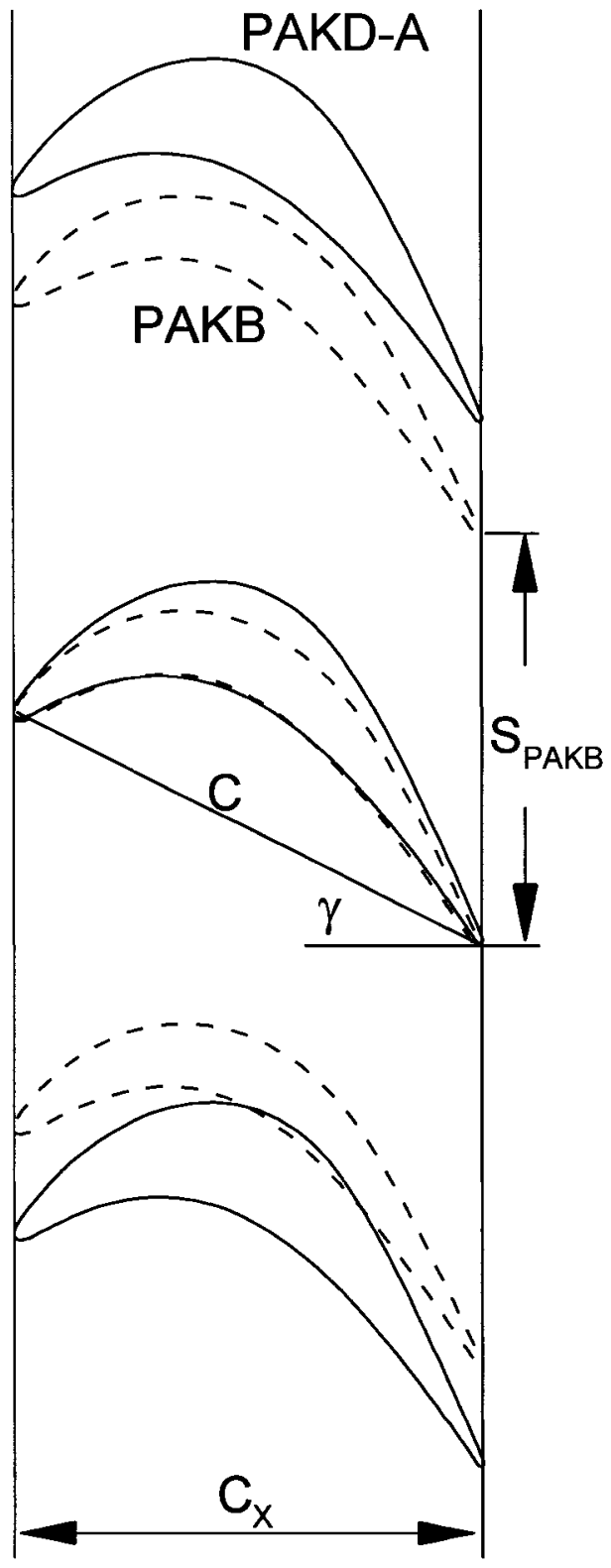

\section{PAKD-F}
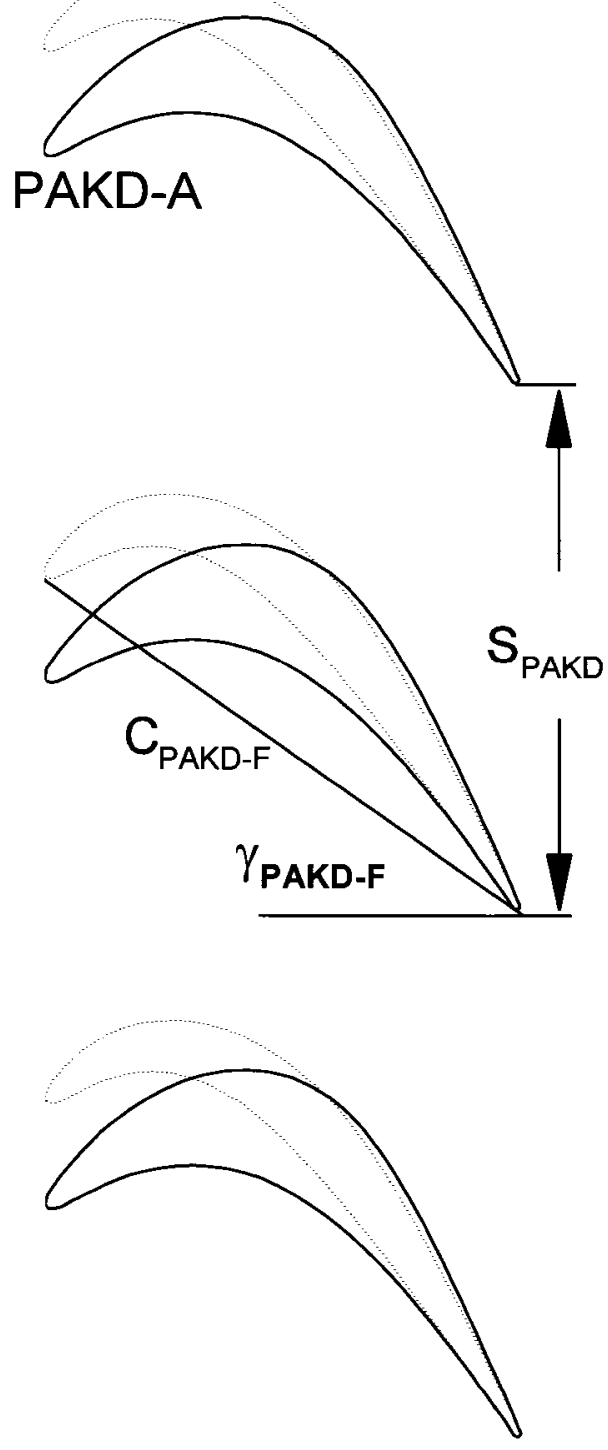

Figure 3.3: Comparison of airfoil geometry 
The wind tunnel has a background FSTI of $0.4 \%$ (Mahallati, 2003). In order to bring the FSTI up to the levels comparable to a realistic engine environment (FSTI of $3-7 \%$, private communications with Pratt \& Whitney Aircraft, East Hartford, CT, 2003), three different turbulence generating grids were utilized. Table 3.2 provides a summary of the grids used for the present measurements. Since the turbulence generating grids have been extensively used in the past, a more detailed description of the design methodology and flow measurements is available in Mahallati (2003) and McAuliffe (2003).

Table 3.2: Summary of Turbulence Generating Grids

\begin{tabular}{|l|c|c|c|}
\hline & Grid I & Grid II & Grid III \\
\hline FSTI & $1.5 \%$ & $4.0 \%$ & $4.0 \%$ \\
\hline$\Lambda$ [mm] & 14 & 8 & 30 \\
\hline Mounting Position & $\mathrm{A}$ & $\mathrm{B}$ & $\mathrm{A}$ \\
\hline
\end{tabular}

As can be seen from the table, both Grid II and III have practically the same FSTI. The main difference between the two grids is in their integral length scales. This is a result of different mounting positions (Figure 3.4) and grid geometry. The drawback of the closely mounted grid (Grid II) is non-uniformity of the inlet flow arising from the fact that the inlet flow near the top of the cascade does not mixout completely before reaching the inlet measurement plane. This will be further addressed in section 4.2.3.

Studies done by Mahallati (2003) and McAuliffe (2003) have shown that a difference in the integral length scale between Grid II and III have very little effect on the results. For the steady part of the experiment, only Grids I and II have been used. However, due to the position of the spoke-wheel, the closely mounted grid (Grid II) could not have been used. Therefore, the unsteady part of the experiment was 


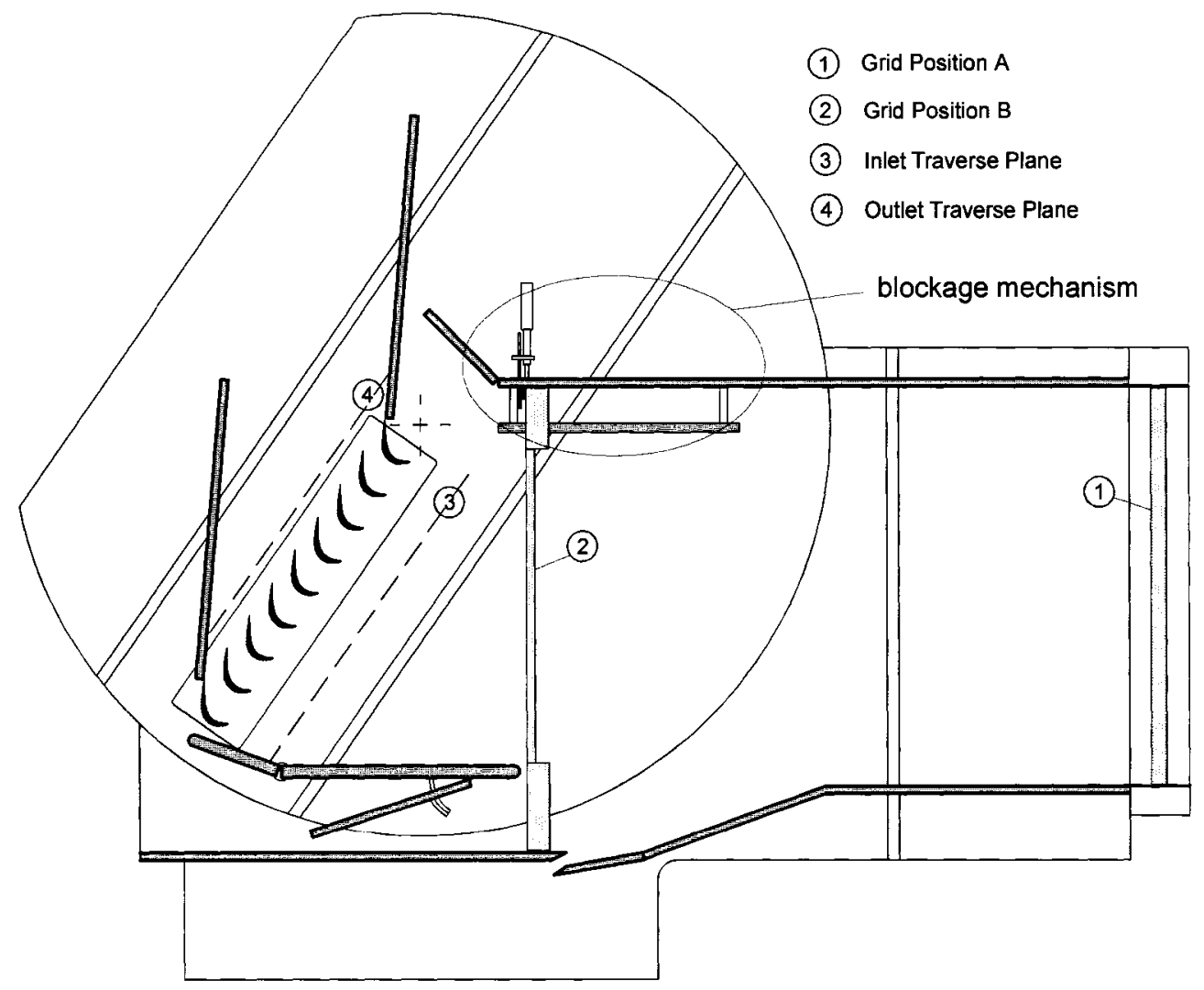

Figure 3.4: Location of Turbulence Generating Grids

(modified from McAuliffe, 2003)

performed using Grid III.

\subsubsection{Wake Generator Rig}

In order to study rotor-stator interactions, a wake generator rig was used to simulate an upstream blade row. The rig was designed and commissioned by Mahallati (2003) and is shown in Figure 3.5. It consists of a frame, a $350 \mathrm{~mm}$ solid wheel with 200 $\mathrm{mm}$ long cylindrical bars (spokes), and a $3 \mathrm{HP}$ AC motor controlled through a solid- 
state frequency controller. The bars were designed to produce the wakes with the same characteristics as a typical upstream blade row. In the present study, both 20 and 60 evenly spaced bars have been used. The rotating plane was positioned at a distance of $60 \%$ axial chord upstream of the leading edge of the cascade. Although the present study examined the unsteady effects at design incidence only, the wheel assembly can be adjusted for off-design measurements using the turntable arrangement similar to the one used for test section.

A photo detector was used to trigger data acquisition during unsteady measurements. Data acquisition was activated once-per-revolution of the spoked wheel using the signal generated by the photo detector. Detailed description of the wake generator system is available in Mahallati (2003).

\subsection{Instrumentation and Probes}

\subsubsection{Pressure Transducers}

Differential pressure transducers were used for pressure measurements. Pressure transducers convert pressure signals into electrical signals. All pressure transducers exhibit a linear relationship between measured pressure differential $\triangle \mathrm{P}$ and their output voltage, $E$, as given by:

$$
\triangle P=C_{1} \cdot E+C_{2}
$$

The calibration coefficients, $\mathrm{C}_{1}$ and $\mathrm{C}_{2}$, are determined from calibration curves (details will be given in section 3.5.1). For the present study 3 types of transducers 

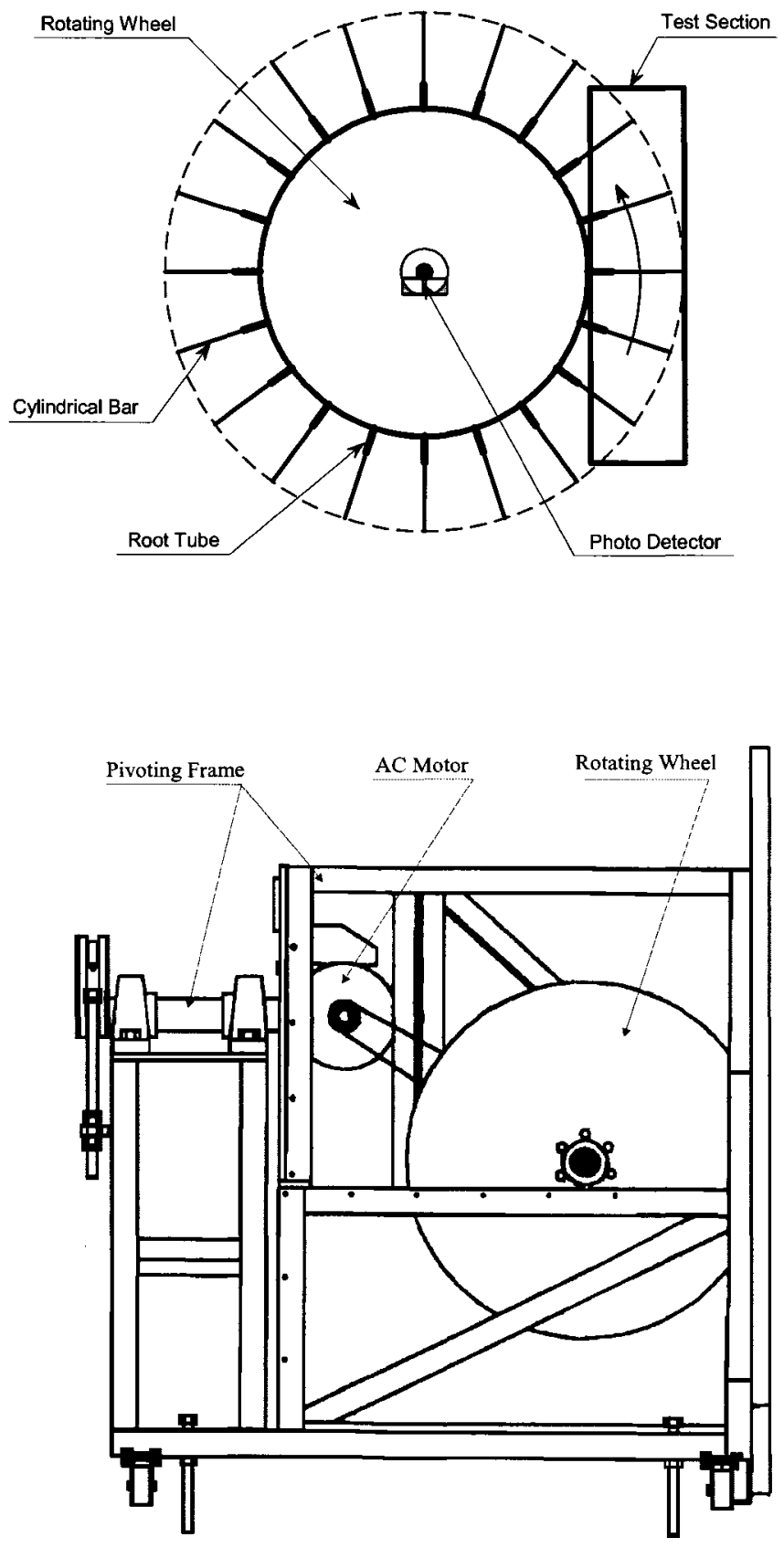

Figure 3.5: Schematic of Spoked-Wheel Wake-Generator Rig

(reproduced from Zhang, 2002) 
were used and their characteristics are summarized in Table 3.3 .

Table 3.3: Pressure Transducers

\begin{tabular}{|c|c|c|}
\hline Transducer & Manufacturer & Pressure Range (Pa) \\
\hline DRAL501DN & Data Instrument & \pm 250 \\
\hline DRAL505DN & Data Instrument & \pm 1250 \\
\hline 113LU20D-PCB & Sensor Technique & \pm 5000 \\
\hline
\end{tabular}

\subsubsection{Temperature Monitor}

The air temperature in the test section for all experiments was recorded using a type-T thermocouple (Copper-Constantan). The thermocouple was connected to an Omega i-series temperature/process meter (model DPi8-C24) with accuracy of $\pm 0.4^{\circ} \mathrm{C}$ (Mahallati, 2003).

The temperature was used to set the operating point of the wind tunnel (Reynolds number) and to correct the hotwire measurements for any temperature differences between calibration and wind tunnel conditions (this will be addressed in sections 4.2.1 and 4.3.2, respectively).

\subsubsection{Digital Barometer}

In addition to temperature, for Reynolds number calculation and thus the operating point setting, the ambient pressure was recorded. The ambient pressure was measured using a digital barometer (Omega model DPI740). The barometer incorporates a pre-calibrated $77-115 \mathrm{kPa}$ (absolute) pressure transducer with accuracy of $\pm 0.02 \%$. The pressure is displayed on the front panel of the barometer and 
transmitted to the computer using the RS-232 port. The pressure readings were recorded continuously for the duration of each experiment.

\subsubsection{Hotwire Anemometry}

An anemometer is an instrument used to infer fluid velocity using a hotwire (or hotfilm sensor). A Lab System Model AN-1003 Anemometry system was used for the hotwire measurements. The role of the anemometer in hotwire measurements is to maintain the temperature of the probe constant at a value which is higher than the temperature of the measured flow through the variation of the hotwire voltage. Hotwire voltage signals are passed through a low-pass filter to remove any high frequency noise. A detailed discussion of the principles of the hotwire anemometry is provided by Brunn (1995).

\subsubsection{Three-hole Probe}

All steady midspan measurements were performed using a three hole pressure probe manufactured by Mahallati (2003). The probe consists of three hypodermic tubes, which are soldered together forming a wedge as shown in Figure 3.6. The width of the probe is $2.4 \%$ and $3.0 \%$ of the blade pitch for PAKD and PAKB, respectively.

The probe was used to measure the total and static pressure, in-plane velocity components and to infer the corresponding flow angle with respect to the probe. Calibration procedure for the probe will be outlined in section 3.5.3. 

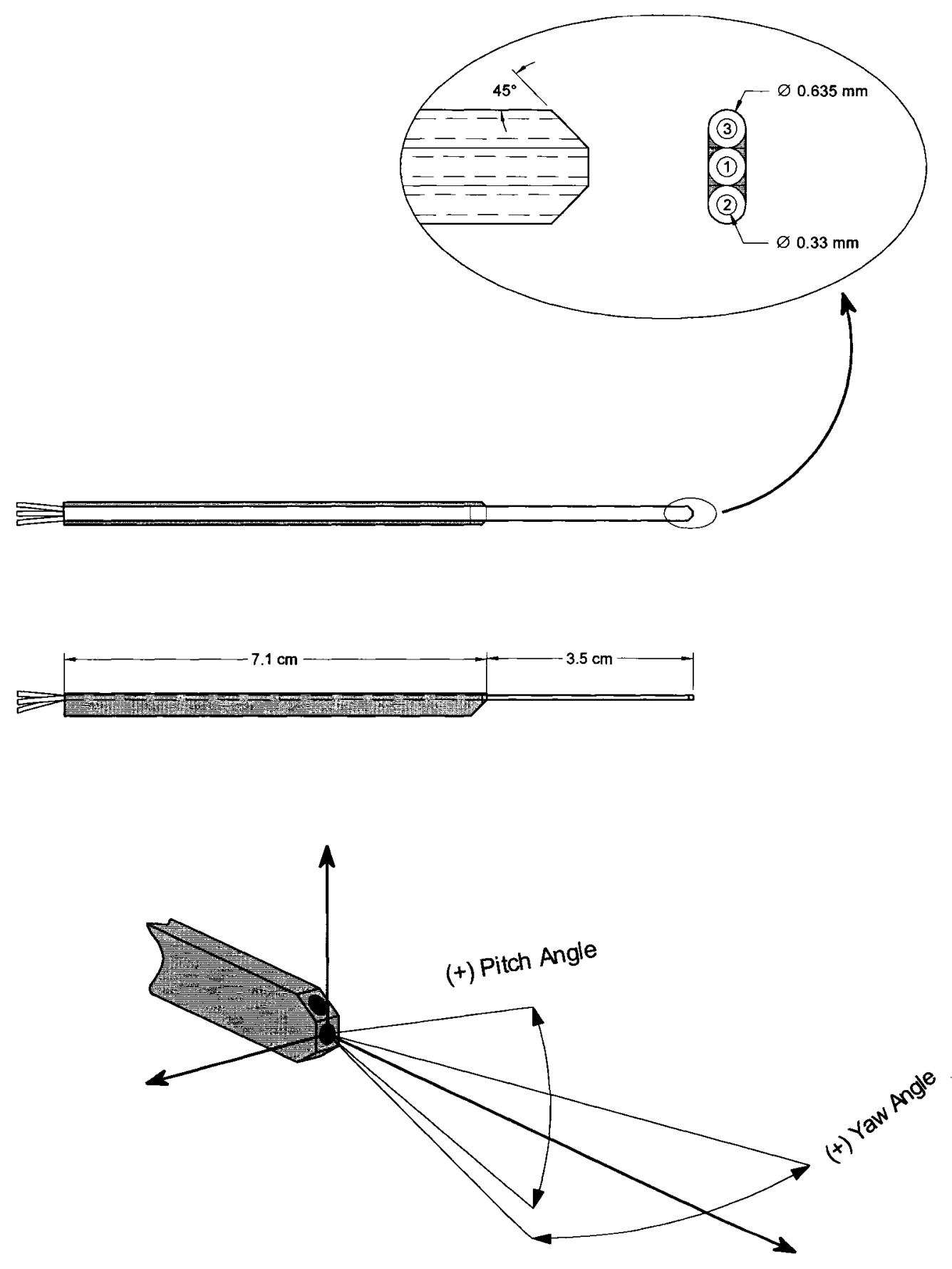

Figure 3.6: Schematic of Three-Hole Probe

(reproduced from Mahallati, 2003) 


\subsubsection{Single-Wire Hotwire Probe}

In the present experiments, a single-wire hotwire probe was used to measure the resultant velocity at the cascade outlet during unsteady measurements. The probe used is a Dantec 55P01, which has gold-plated tungsten wire with a diameter of $5 \mu \mathrm{m}$ and length of $3 \mathrm{~mm}$ (active sensor length is $1.25 \mathrm{~mm}$ ). The advantage of plated probes is in minimizing aerodynamic disturbances caused by the prongs.

The hotwire was frequently calibrated during the unsteady part of the experiment. Calibration procedure is given in section 3.5.4.

\subsection{Data Acquisition System}

The Data acquisition system used in the present investigation consists of a PowerDAQ II data acquisition board and the CarletonDAQ (initially named TurboDAQ), Windows based object-oriented software package developed and tested by Mahallati (2003).

PowerDAQ II board is a sample-and-hold PCI card installed on a personal computer. The board was manufactured by United Electronics Industries (UEI), model PD2-MFS-8-800/14. It was used to acquire the electronic signals from the pressure transducers and anemometer, and stream them to the hard disk. The maximum sampling frequency of the board is $800 \mathrm{kHz}$.

The CarletonDAQ software was used for data acquisition, device set-up and threehole probe data reduction and calibration. During the unsteady experiments an external trigger was used to trigger data acquisition once per revolution of the spoke 
wheel.

More details on both the board and CarletonDAQ software were given in Mahallati (2003).

\subsection{Calibration Apparatus and Methods}

\subsubsection{Pressure Transducer Calibration}

The pressure transducers were calibrated using a water micromanometer (details are available from Benner, 2003). As already discussed in section 3.3.1, the transducers exhibit a linear relationship between the measured pressure differential and their output voltage. The goal of calibration was to determine the slope of the resulting

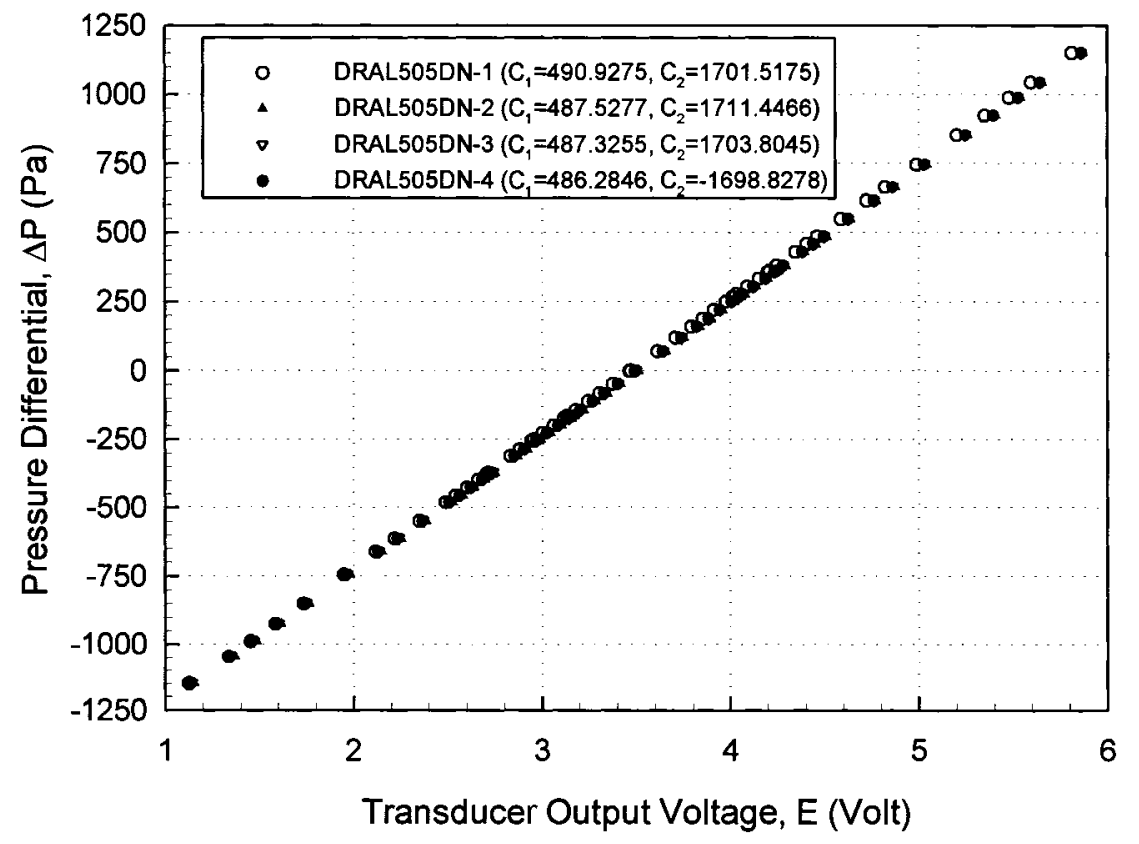

Figure 3.7: DRAL505DN Calibration Curves 
curve $\left(\mathrm{C}_{1}\right)$, while the zero offset $\left(\mathrm{C}_{2}\right)$ was adjusted on a daily basis. Calibrations were performed at least once per month. For every calibration a set of at least 50 points was used covering the full range of transducer operation. Typical calibration curves for DRAL501DN are shown in Figure 3.7.

\subsubsection{Calibration Apparatus}

The calibration of the pressure and hotwire probes was performed in the in-house calibration rig. It is an open-circuit, suction type calibration rig capable of achieving velocities of up to $45 \mathrm{~m} / \mathrm{s}$. A throttle valve located upstream of the blower is used to

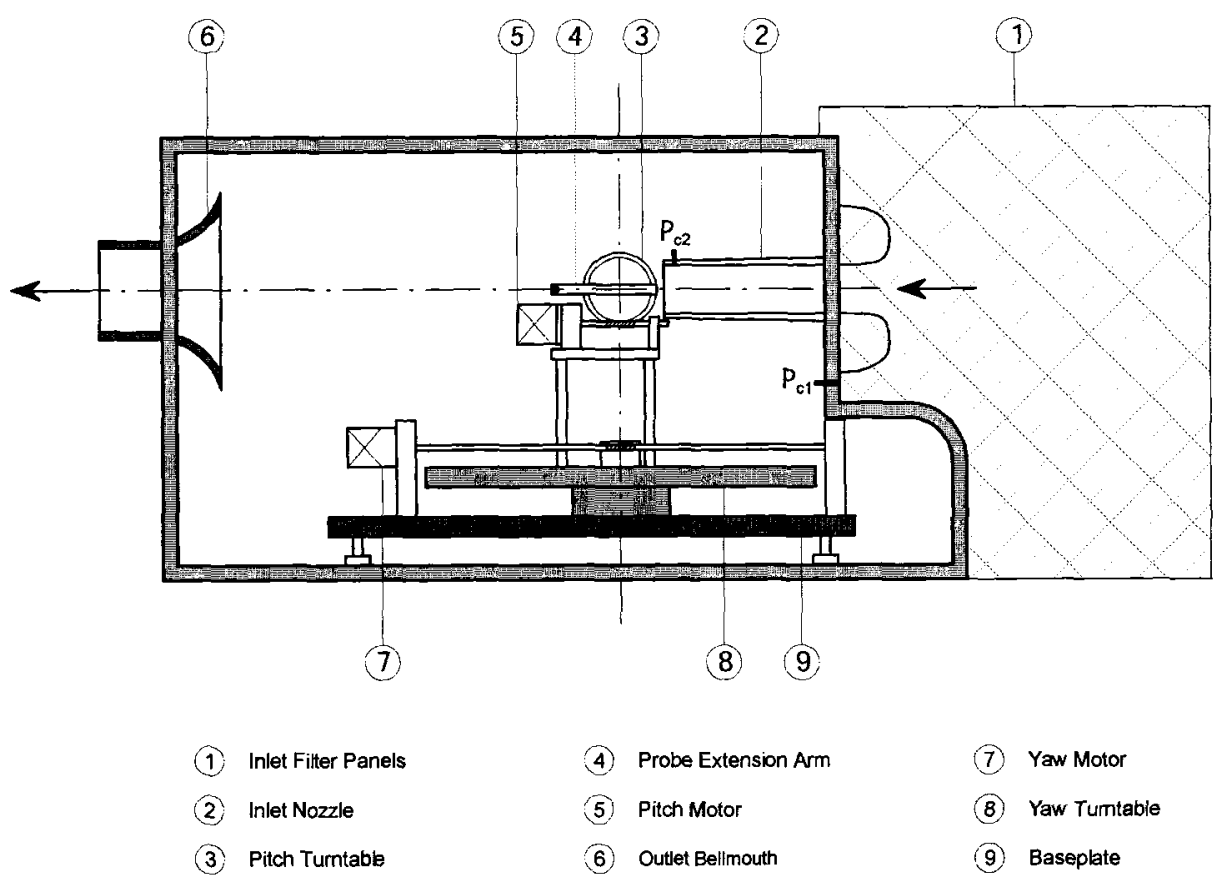

Figure 3.8: Schematic Drawing of Calibration Rig

(reproduced from Mahallati, 2003) 
control the airflow through the outlet bellmouth.

The calibration rig is equipped with two motorized turntables allowing variations in both pitch and yaw over a range of $\pm 50^{\circ}$. The turntables are driven with stepper motors similar to the ones used for linear traversing of the probes at the cascade inlet and outlet. CarletonDAQ and the same motor controller are used to drive the motors. Total and static reference pressures, $\mathrm{P}_{C 1}$ and $\mathrm{P}_{C 2}$ are measured at locations shown in the Figure 3.8.

\subsubsection{Three-hole Probe Calibration Procedure}

Three hole probe calibration was performed in the previously described calibration rig. First, the probe was aerodynamically aligned with the flow, and then it was varied over a range of pitch angles $\left( \pm 20^{\circ}\right.$ in increments of $\left.0.5^{\circ}\right)$. The method outlined by Lewis (1966) was used. Calibration file consists of 6 coefficients for each calibration angle resulting in 6 calibration curves shown in Figure 3.9. Calibration coefficients were determined from the following equations:

$$
\begin{aligned}
& K_{1}=\frac{P_{1}-P_{S}}{P_{0}-P_{S}}=C_{p 1} \\
& K_{12}=\frac{P_{1}-P_{2}}{P_{0}-P_{S}}=C_{p 1}-C_{p 2} \\
& K_{13}=\frac{P_{1}-P_{3}}{P_{0}-P_{S}}=C_{p 1}-C_{p 3} \\
& K_{23}=\frac{P_{2}-P_{3}}{P_{0}-P_{S}}=C_{p 2}-C_{p 3} \\
& K_{\phi 2}=\frac{P_{1}-P_{2}}{P_{0}-P_{S}}=\frac{K_{12}}{K_{13}}
\end{aligned}
$$




$$
K_{\phi 3}=\frac{P_{1}-P_{2}}{P_{0}-P_{S}}=\frac{1}{K_{\phi 2}}
$$

An extensive study investigating the sensitivity in Reynolds number and yaw angle misalignment was undertaken by Mahallati (2003). He found that small variation in Reynolds number does not affect the results greatly and can be incorporated into the quoted uncertainties. In addition, he also concluded that the probe is insensitive to yaw angle misalignments of $\pm 5^{\circ}$.

Since the present study covered a wide range of Reynolds numbers, the calibration was performed for velocities varying from $5 \mathrm{~m} / \mathrm{s}$ to $45 \mathrm{~m} / \mathrm{s}$. This range of velocities corresponds to the range of mass averaged velocities encountered in the cascade. The calibration curves corresponding to the velocity of $25 \mathrm{~m} / \mathrm{s}$ (a typical outlet velocity for off-design part of the study) are shown in Figure 3.9

\subsubsection{Single-Wire Hotwire Calibration}

The hotwire calibration was performed in the same rig as three-hole probe calibration. First the probe was aligned with the incoming flow from the jet and then the velocity in the test section was varied from 1 to $45 \mathrm{~m} / \mathrm{s}$.

A typical calibration consisted of 30 points covering the full range of velocities. The calibration data was fitted using King's law:

$$
E_{T O B}^{2}=A+B U^{n}
$$

where $\mathrm{A}, \mathrm{B}$ and $\mathrm{n}$ are constants and $E_{T O B}^{2}$ and $U$ are voltage and flow velocity respectively. A typical calibration curve is shown in Figure 3.10. 

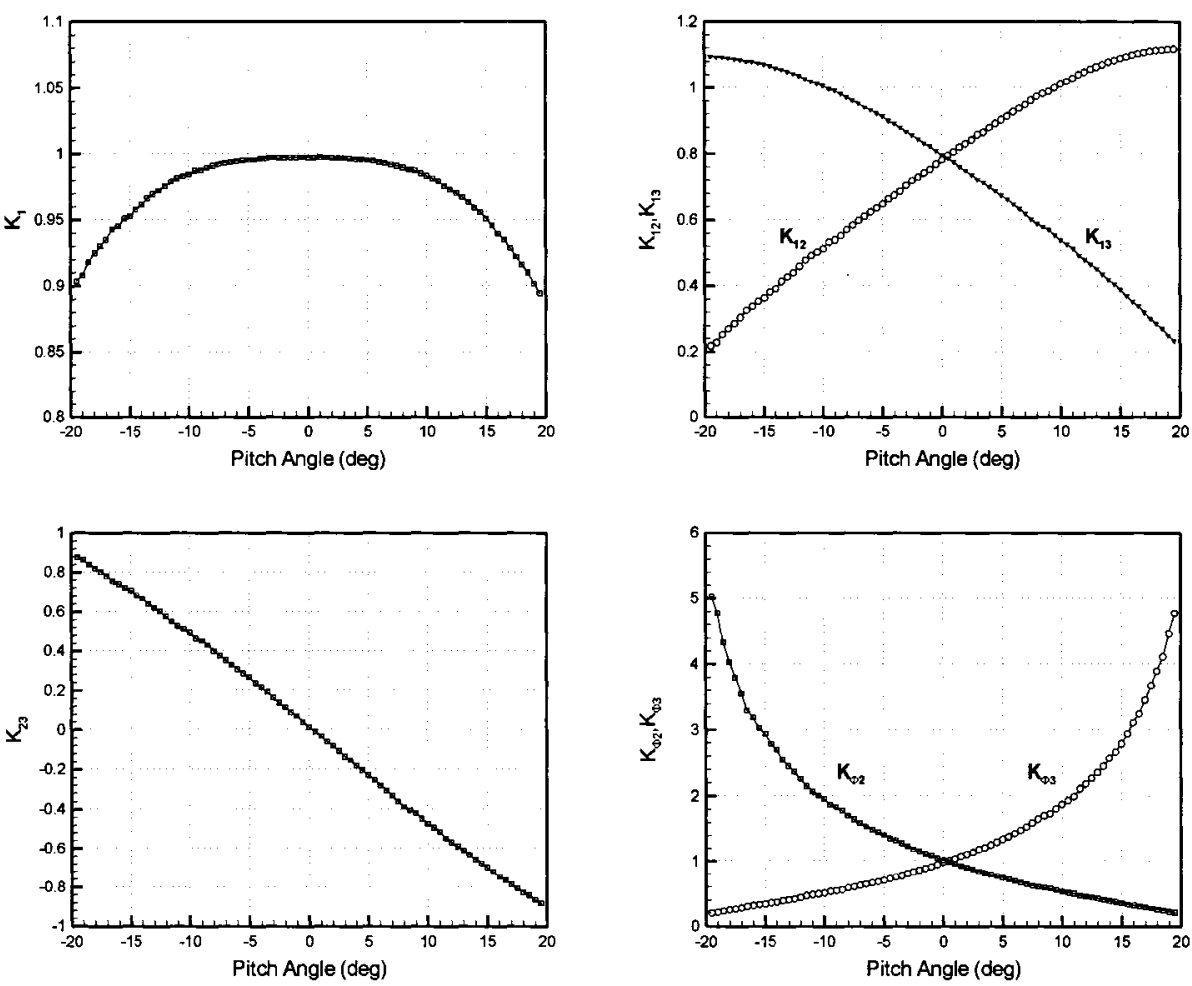

Figure 3.9: Three-hole Probe Calibration Curves

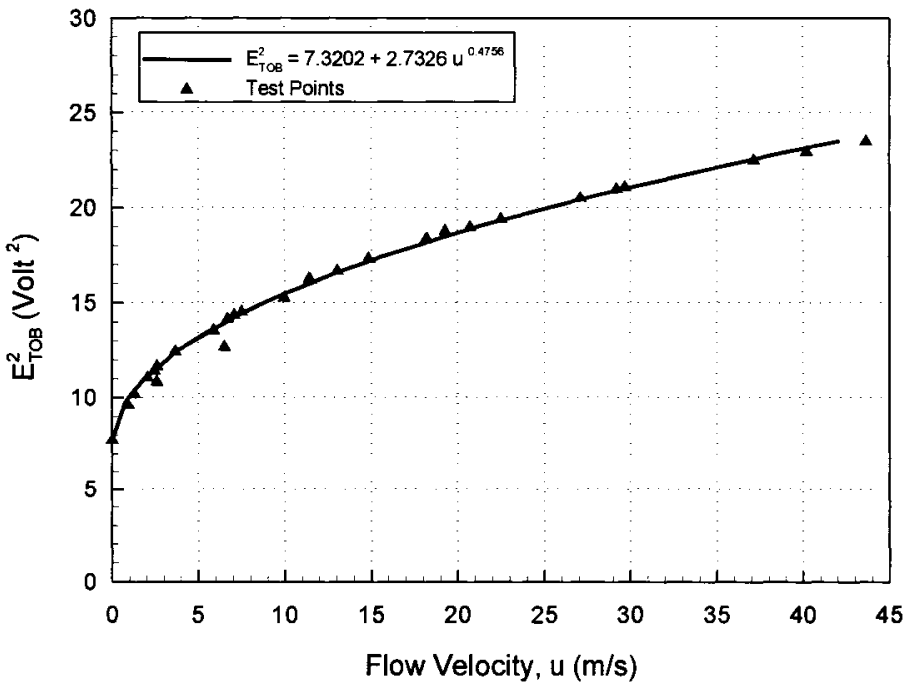

Figure 3.10: Hotwire Calibration Curve 


\section{Chapter 4}

\section{Experimental Procedures and Data Reduction}

\subsection{Introduction}

The experimental procedures, data reduction and uncertainty analysis will be described in this chapter. Section 4.2 will present the steady flow measurement procedures and data reduction. In Section 4.3, the unsteady measurements and data reduction will be discussed. Finally, the chapter will end with a brief description of uncertainty analysis. 


\subsection{Experimental Procedures and Data Reduction for Steady Flow Measurements}

\subsubsection{Wind Tunnel Operating Point}

The wind tunnel operating point was set by adjusting the fan's rotational speed to give a desired value of Reynolds number. Reynolds number based on the outlet velocity, $R e_{e}$, defined in Eq. 4.1, is the most appropriate form of the Reynolds number, since the profile losses scale on and are normalized by the outlet dynamic pressure. $R e_{e}$ is not known before the detailed measurements at inlet and outlet are performed, and the velocity ratio, $U_{e} / U_{i}$, has been calculated. However, detailed measurements cannot be performed before the wind tunnel operating point is set and inlet flow uniformity and outlet flow periodicity are achieved. Obviously, this makes the procedure of setting the operating point setting procedure iterative and time consuming. Fortunately, for a fixed incidence, the velocity ratio does not change much with the Reynolds number as long as the flow remains attached at the trailing edge of the blade. As seen from Eq. 4.1 and 4.2, the $R e_{e}$ and $R e_{i}$ (Reynolds number based on the inlet velocity, defined in Eq. 4.2) are directly related through the velocity ratio. Therefore, to set the wind tunnel operating point, $R e_{i}$ was used for practical reasons.

$$
\begin{gathered}
R e_{e}=\frac{\rho U_{e} C_{x}}{\mu}=\frac{\rho U_{i} C_{x}}{\mu} \cdot \frac{U_{e}}{U_{i}}=R e_{i} \cdot \frac{U_{e}}{U_{i}} \\
R e_{i}=\frac{\rho U_{i} C_{x}}{\mu}
\end{gathered}
$$


The wind tunnel operating point is monitored through the contraction pressure difference, $P_{C 1}-P_{C 2}$, as discussed in Section 3.2.1. $P_{C 1}$ is close to the stagnation pressure in the settling chamber and $P_{C 2}$ is the static pressure at the test section inlet. The actual inlet dynamic pressure at the cascade, $Q_{i}^{\prime}$, is related to the contraction pressure difference through the following relation:

$$
C_{Q W T}=\frac{Q_{i}^{\prime}}{P_{C 1}-P_{C 2}}
$$

where $C_{Q W T}$ is the wind tunnel calibration coefficient. It is obtained from the threehole probe traverses at the cascade inlet at midspan. However, $C_{Q W T}$ is not known at the beginning of the procedure. To speed up the procedure, an old value for the same cascade or grid was used as the starting point, until a more accurate value was obtained. Thus, the inlet velocity is equal to:

$$
U_{i}=\sqrt{\frac{2 C_{Q W T}\left(P_{C 1}-P_{C 2}\right)}{\rho}}
$$

where density, $\rho$, was obtained from the ideal gas law based on the test section temperature and the atmospheric pressure. The temperature and ambient pressure were measured using a thermocouple and digital barometer as described in Sections 3.3 .2 and 3.3 .3 , respectively.

\subsubsection{Three-hole Probe Measurements}

The three-hole pressure probe shown in Section 3.3.5 was used for midspan measurements at both inlet and outlet planes. The data reduction is incorporated into the CarletonDAQ software described in Section 3.4. In order to reduce the data, 
a calibration file with the tabulated three-hole probe calibration coefficients has to be specified by the user. The calibration coefficients $K_{1}, K_{12}, K_{13}, K_{23}, K_{\phi 2}, K_{\phi 3}$ were defined in Section 3.5.3.

The outputs of the data reduction code are the measured values of static $\left(C_{P S}\right)$, dynamic $\left(C_{Q}\right)$ and total $\left(C_{P 0}\right)$ pressure coefficients, velocity coefficient $\left(C_{V}\right)$ as well as the flow angle $(\Phi)$ relative to the probe. An excellent overview of three-hole probe data reduction is available from McAuliffe (2003). Therefore, only the coefficients will be defined here. In the following expressions, $P_{0}$ and $P_{S}$ are the total and dynamic pressures, respectively.

$$
\begin{gathered}
C_{Q}=\frac{P_{0}-P_{S}}{P_{0 r e f}-P_{\text {Sref }}} \\
C_{V}=\sqrt{C_{Q}} \\
C_{P S}=\frac{P_{S}-P_{\text {Sref }}}{P_{0 r e f}-P_{S r e f}} \\
C_{P 0}=\frac{P_{0 r e f}-P_{0}}{P_{0 r e f}-P_{\text {Sref }}}=1-C_{P S}-C_{Q}
\end{gathered}
$$

The reference conditions, ref, were $P_{C 1}$ (for total pressure) and $P_{C 2}$ (for static pressure) for cascade inlet measurements, and $P_{0 i}$ (actual inlet total pressure) and $P_{S i}$ (actual inlet static pressure) for cascade outlet measurements, respectively.

As previously mentioned, $\Phi$ is the flow angle relative to the probe. The inlet and 
outlet flow angles can be found from:

$$
\begin{aligned}
& \alpha_{i}=\varphi_{i}+\Phi \\
& \alpha_{e}=\varphi_{e}+\Phi
\end{aligned}
$$

where $\varphi$ is the probe angle relative to the axial direction. For all measurements, the three-hole probe was aligned with the expected inlet and outlet flow vectors to minimize any inaccuracies arising from large misalignments of the probe relative to the measured flow.

\subsubsection{Inlet Flow Uniformity and Outlet Flow Periodicity}

The blades in an axial flow turbine are distributed evenly around the circumference. Thus, they operate as an infinite blade row with periodic flow conditions. In order to simulate actual machine conditions with a finite number of linear-cascade blades, the control surfaces described in Section 3.2.2 are used to achieve inlet flow uniformity and outlet flow periodicity. It is not possible to achieve flow periodicity for the end blades of the cascade. However, the middle blades can create essentially periodic flow provided that the control surfaces are set properly.

For each operating point/condition, the control surfaces were first set to the positions that were known to produce uniform and periodic flow for a similar Reynolds number and turbulence generating grid. The procedure for setting flow uniformity and periodicity usually took a number of iterations per case, since the adjustments of the control surfaces to achieve outlet flow periodicity also affect inlet flow uniformity, 
and vice versa.

The inlet and outlet traverses were performed with the three-hole probe at 1.2 $C_{x}$ upstream and $0.4 C_{x}$ downstream of the leading and trailing edges, respectively, as shown in Figure 4.1. The traverse distance in the pitchwise direction for the two PAKD airfoils corresponds to four blade pitches. Since PAKB has a smaller pitch, for those tests the traverse distance corresponds to five pitches. Figures 4.2 and 4.3 show both the inlet flow uniformity and outlet flow periodicity with the freestream turbulence intensity (FSTI) of $1.5 \%$ and $4 \%$ for one typical case (PAKD-F at a Reynolds number of 150,000 and design incidence). The flow parameters shown are the inlet and outlet flow angles, dynamic, static and total pressure coefficients as defined in the previous section.

It should be noted that due to the proximity of the grid used for the $4 \%$ FSTI measurements (Grid II), the top portion of the inlet flow (low values of $y / s$ in Figure 4.3) was influenced by the wakes coming from the grid. Since the cascade is inclined relative to the grid, the top part of the cascade is closer to the grid than the bottom part, as can be seen from Figure 4.1. As a consequence, the wakes of the grid bars near the top of the cascade have not mixed-out fully before reaching the inlet measurement plane at $1.2 C_{x}$. As can be seen from the outlet total pressure coefficient $C_{P 0 e}$ (Figure 4.3 , bottom right hand side), the mixing continues through the top blade passages and seems to be complete when the flow reaches the outlet traverse plane. As a result of the decay of the inlet flow non-uniformities through the upper blade passages, a small pressure loss is evident in the "freestream" flow (the flow outside of the blade wakes) for the most upper blade passage presented in Figure 4.3. However, the middle and lower portion of the inlet flow seem to be nearly mixed-out already at the inlet plane, thus there is very little if any additional mixing through the middle and lower 


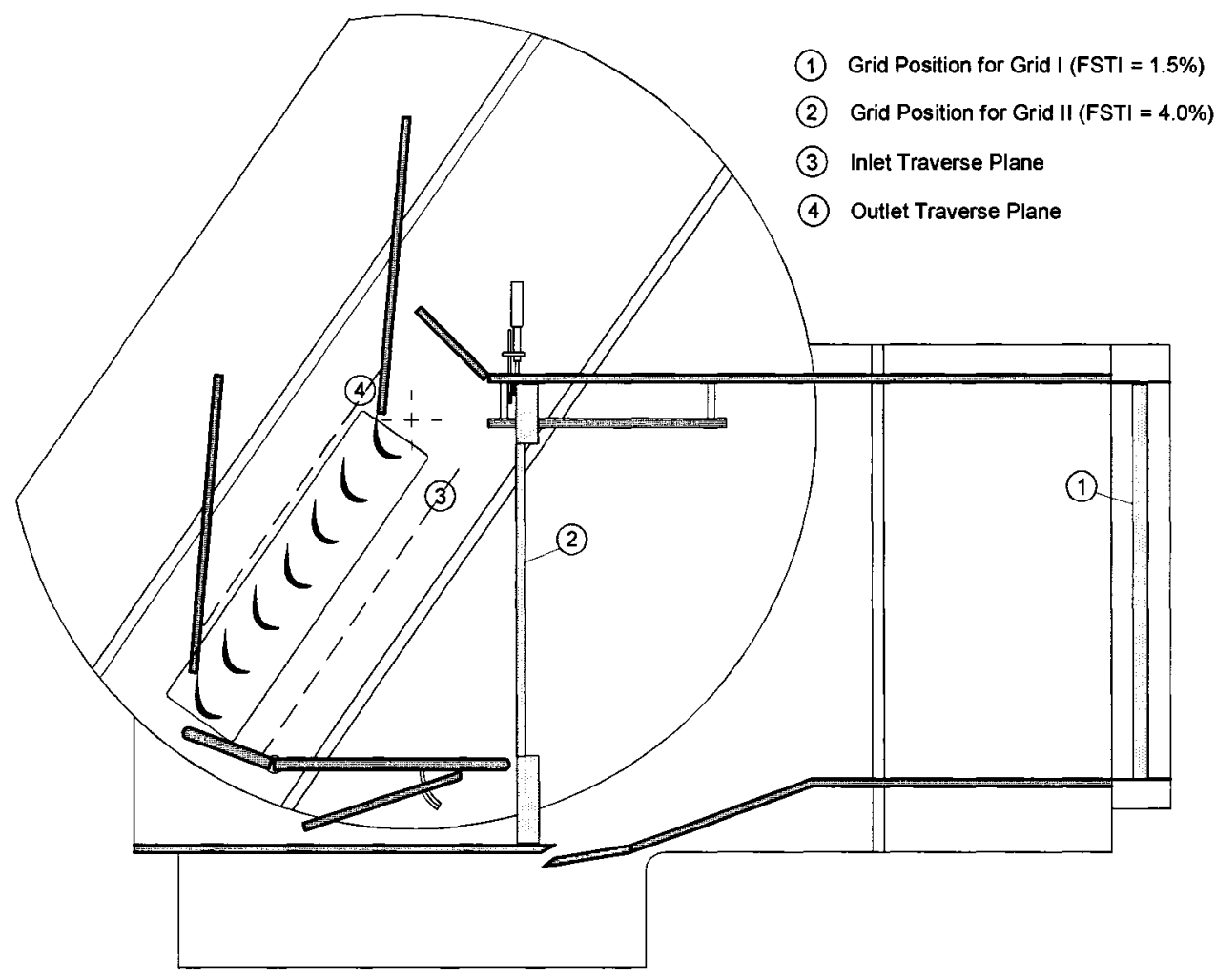

Figure 4.1: Locations of the Grids and Measurement Planes (modified from McAuliffe, 2003)

blade passages. Since the profile losses will be presented for the middle blade only, the non-uniformities do not affect the presented losses noticeably. Similar findings were reported by McAuliffe (2003), who designed and commissioned Grid II.

After the flow uniformity and periodicity were established, more detailed measurements with points spaced approximately $1.5 \%$ of the blade pitch were performed at the downstream traverse plane to measure the profile loss, outlet flow angle, velocity ratio and axial velocity ratio. These quantities will be defined in the following two sections. It should be noted that the detailed results that will be presented in the thesis were measured for the middle blade only for all cascades and operating conditions. 

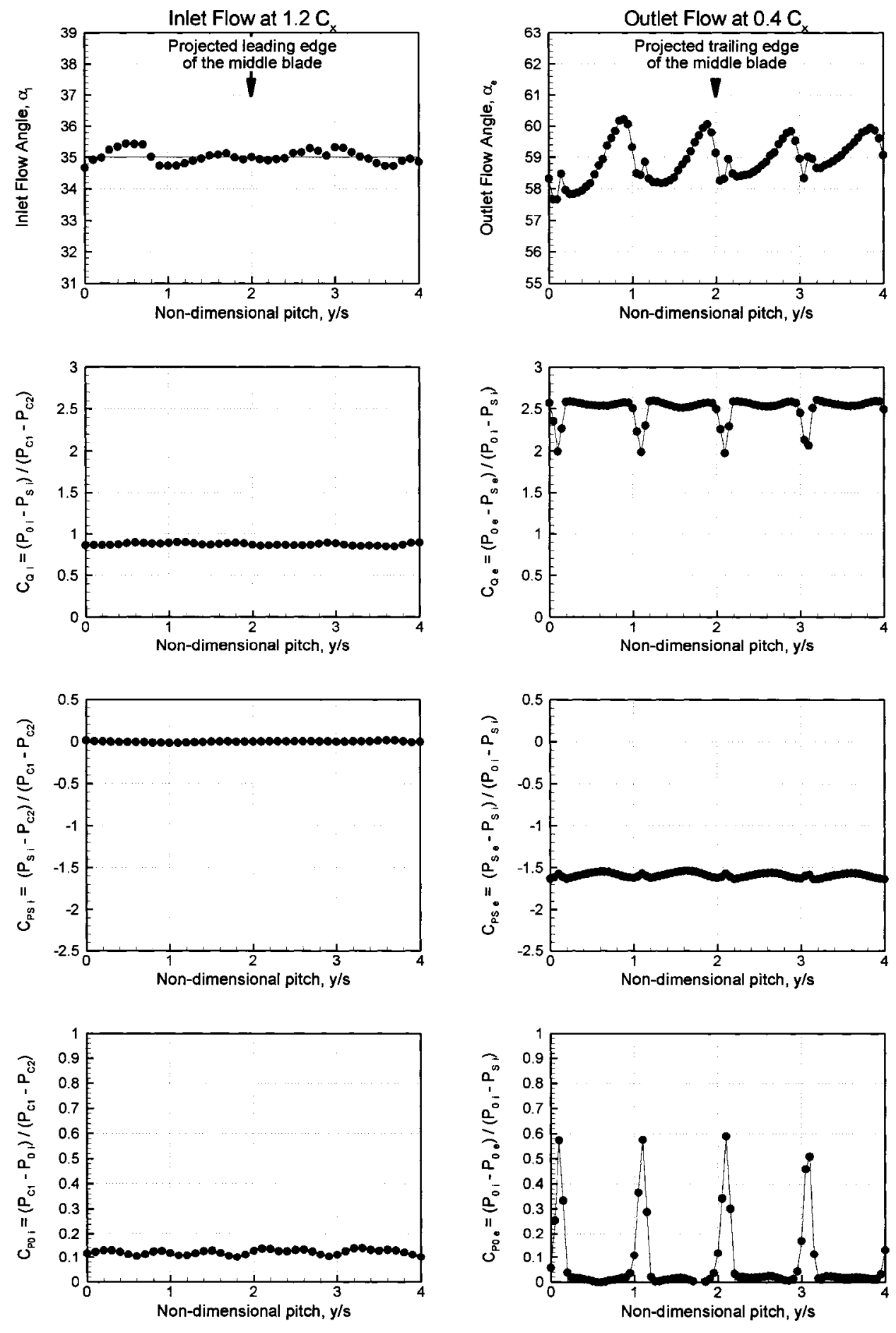

Cascade Top

Cascade Bottom

Cascade Top

Cascade Bottom

Figure 4.2: Inlet Flow Uniformity and Outlet Flow Periodicity

(PAKD-F: $\mathrm{Re}=150,000, \mathrm{FSTI}=1.5 \%$ and Design Incidence) 

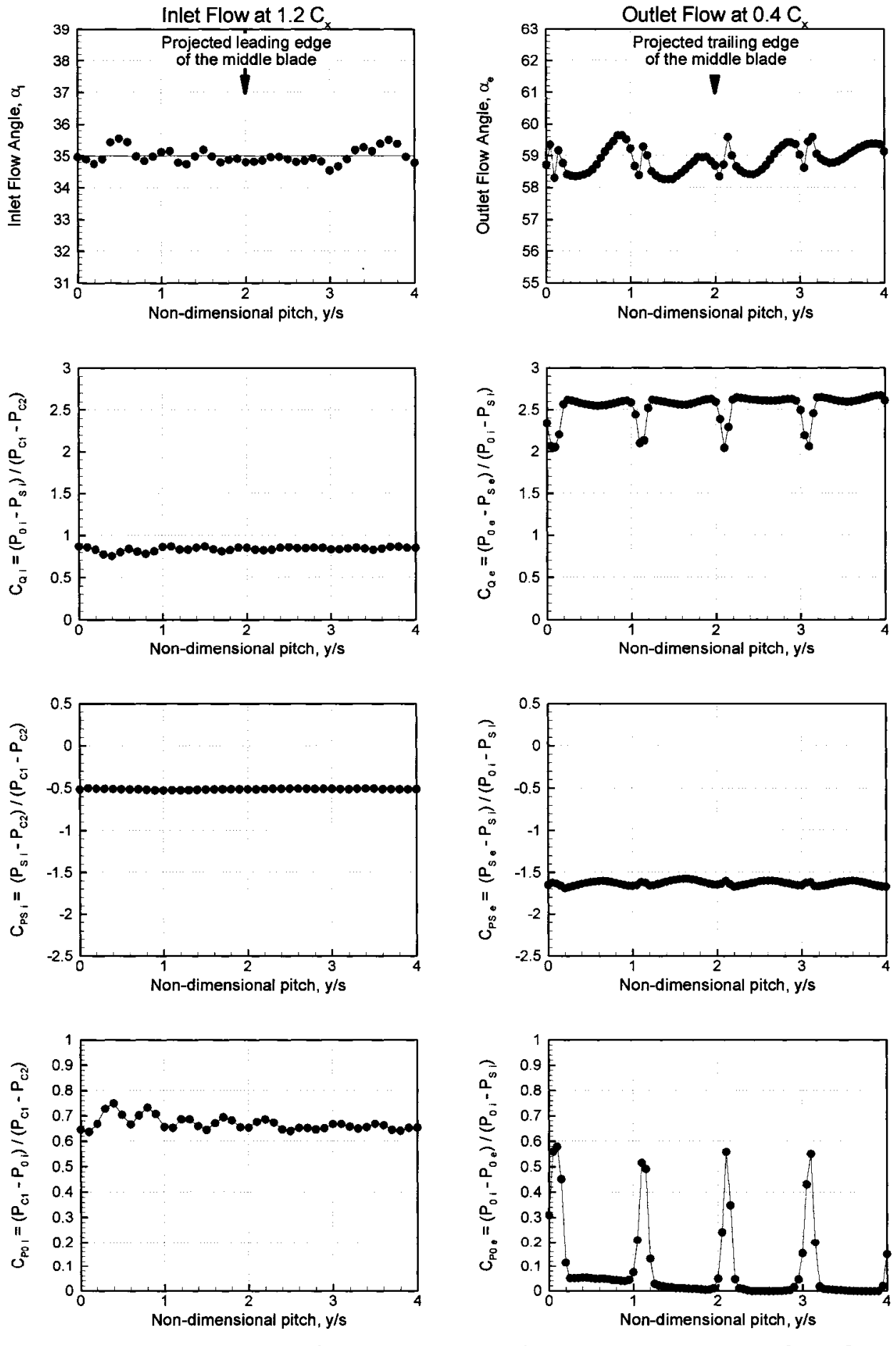

Cascade Top

Cascade Bottom

Cascade Top

Cascade Bottom

Figure 4.3: Inlet Flow Uniformity and Outlet Flow Periodicity

(PAKD-F: $\operatorname{Re}=150,000, F S T I=4 \%$ and Design Incidence) 


\subsubsection{Two Dimensionality of the Midspan Flow}

The axial velocity-density ratio (AVDR) is a measure of two-dimensionality of the midspan flow. It is defined as the ratio of outlet-to-inlet mass flow rates per unit span. The current measurements were performed at essentially incompressible conditions, since the maximum outlet velocities at the highest Reynolds number examined $(150,000)$ correspond to about $50 \mathrm{~m} / \mathrm{s}$ (Mach number of approximately 0.15). Therefore, it is possible to omit the density term and use the axial velocity ratio (AVR) as the measure of stream tube contraction. The AVR is thus defined as the ratio of outlet-to-inlet axial velocity:

$$
A V R=\frac{U_{x e}^{\prime}}{U_{x i}^{\prime}}=\frac{\int_{0}^{1} U_{e} \cos \left(\alpha_{e}\right) d(y / s)}{\int_{0}^{1} U_{i} \cos \left(\alpha_{i}\right) d(y / s)}
$$

Values of AVR greater than 1.0 imply that the flow rate per unit span at the midspan is greater at the outlet than at the inlet, and thus there is a contraction in the streamtube through the blade passage, and vice versa. A study by Rodger (1992) examined the influence of the AVR on the midspan performance (both losses and flow angles). He pointed out that AVR is a necessary, but not sufficient condition for flow two-dimensionality, since it is only a measure of the average streamtube convergence. He also showed that the values of the AVR greater than 1.0 have smaller effect on the airfoil performance than values lower than 1.0. In Sections 5.3.3 and 6.3, the values of AVR for the current measurements will be presented and discussed. 


\subsubsection{Mixed-out Profile Loss and Flow Angle}

The measured loss coefficient (total pressure loss between inlet and outlet planes normalized by the outlet dynamic pressure) is dependent on the location of the planes of measurements, since downstream of the blades the losses are still being generated through the mixing of the blade wakes with the "freestream" flow. To be able to compare the results obtained from different cascades, which might have been measured at different planes, the mixed-out losses are commonly used. The mixed-out plane, $m$, is a hypothetical plane located infinitely far downstream from the trailing edge, where the flow is fully mixed-out, as shown in Figure 4.4. It is assumed that the mixing occurs in constant area under incompressible conditions. For stationary blades, the mixed-out loss coefficient, $Y_{m}$, is defined as:

$$
Y_{m}=\frac{P_{0 i}^{\prime}-P_{0 m}^{\prime}}{P_{0 m}^{\prime}-P_{S m}^{\prime}}
$$

The derivation and adaptation of the mixing-out equations for the current experimental set-up was done by Mahallati (2003) and only the final forms of the equations will be given here.

$$
Y_{m}=\frac{1-\int_{0}^{1} C_{P S e} d(y / s)+2\left(I_{1}^{2}-I_{2}\right)}{\left(\frac{U_{m}}{U_{i}}\right)^{2}}-1
$$

where $C_{P S e}$ is the exit static pressure coefficient from the three-hole probe measurements, defined in Eq. 4.7, the velocity ratio $U_{m} / U_{i}$, and the integrals $I_{1}$ and $I_{2}$ are defined as follows: 

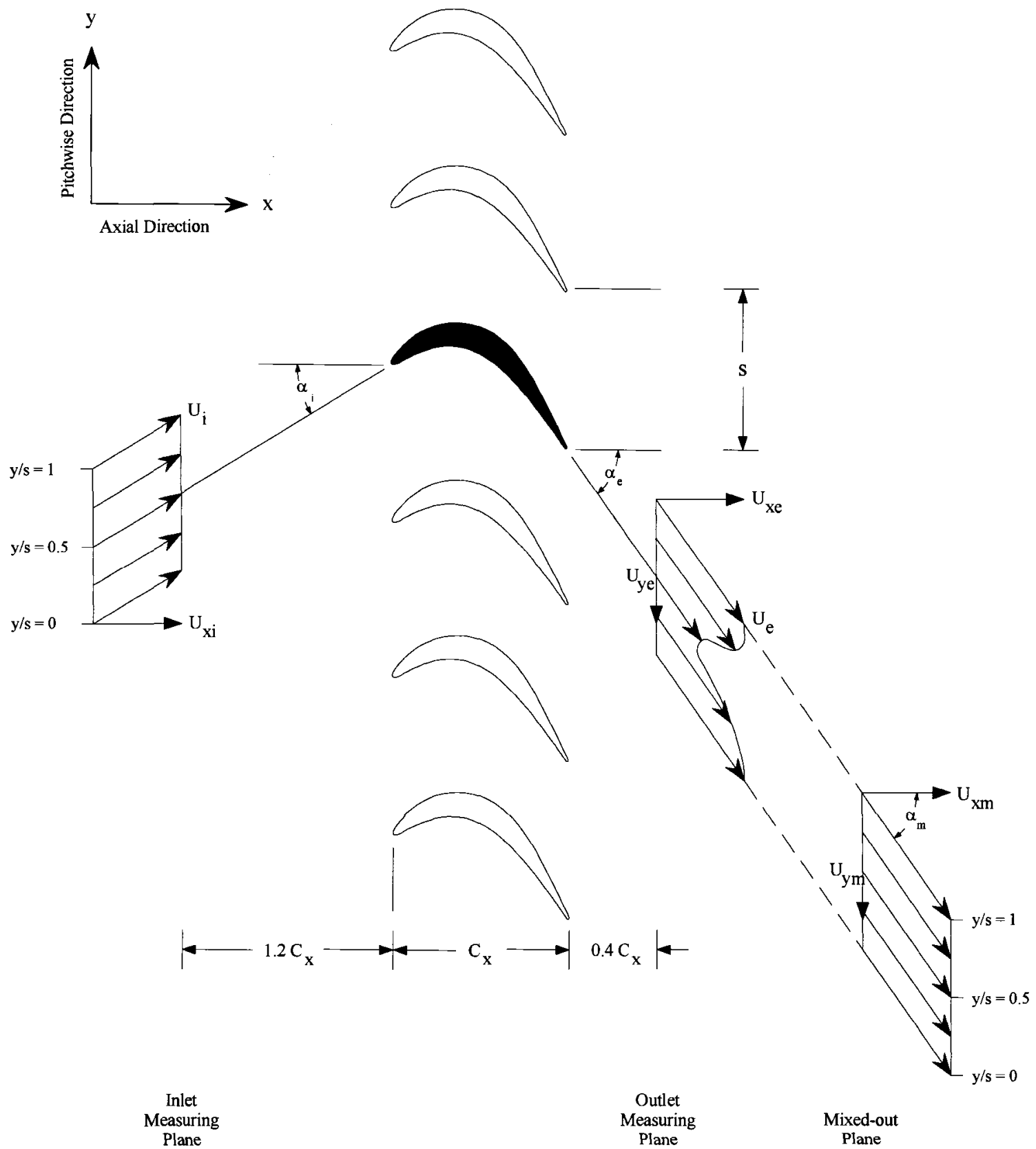

Figure 4.4: Measurement Planes and Nomenclature for Steady Flow

(modified from Mahallati, 2003) 


$$
\begin{gathered}
\left(\frac{U_{m}}{U_{i}}\right)^{2}=I_{1}^{2}+\left[\frac{\int_{0}^{1}\left(\frac{U_{e}}{U_{i}}\right)^{2} \sin \left(2 \alpha_{e}\right) d(y / s)}{2 I_{1}}\right]^{2} \\
I_{1}=\int_{0}^{1}\left(\frac{U_{e}}{U_{i}}\right) \cos \alpha_{e} d(y / s) \\
I_{2}=\int_{0}^{1}\left(\frac{U_{e}}{U_{i}}\right)^{2} \cos \alpha_{e}^{2} d(y / s)
\end{gathered}
$$

The mixed-out flow angle can be found from:

$$
\alpha_{m}=\arctan \left(\frac{U_{y m}^{\prime}}{U_{x m}^{\prime}}\right)=\arctan \left[\frac{\frac{\int_{0}^{1}\left(U_{e}\right)^{2} \sin \left(2 \alpha_{e}\right) d(y / s)}{2 \int_{0}^{1} U_{e} \cos \left(\alpha_{e}\right) d(y / s)}}{\int_{0}^{1} U_{e} \cos \left(\alpha_{e}\right) d(y / s)}\right]
$$

\subsubsection{Blade Loading Measurements}

There is a close relationship between the blade static pressure distribution (loading) and the development and possible separation of the boundary layer, as discussed in Chapter 2. In order to conduct loading measurements, the middle blade(s) were replaced with instrumented blades, which have static pressure taps placed over the airfoil surface. Pressure tap positions are given in Appendix A, and Table 4.1 summarizes the number of pressure taps for each airfoil.

The loading measurements were made after the inlet flow uniformity and outlet 
Table 4.1: Summary of Pressure Tap Numbers for Loading Measurements

\begin{tabular}{|c|c|c|c|}
\hline & PAKB & PAKD-A & PAKD-F \\
\hline Suction side & 25 & 32 & 26 \\
\hline Pressure side & 15 & 17 & 20 \\
\hline
\end{tabular}

flow periodicity were established. Two alternative definitions of pressure coefficient for loading measurements were used in the present work. Appendix B provides a discussion of the loading coefficients and their respective advantages and disadvantages. The first coefficient, used for all loading measurements at design incidence presented in Chapter 5, uses the inlet dynamic pressure as the normalizing pressure difference and is given by:

$$
C_{P l Q i}=\frac{P_{0 i}^{\prime}-P_{S l}}{P_{0 i}^{\prime}-P_{S i}^{\prime}}=1+\frac{1}{C_{Q i}^{\prime}}+\frac{C_{P S i}^{\prime}}{C_{Q i}^{\prime}}+\frac{P_{C 1}-P_{S l}}{C_{Q i}^{\prime}\left(P_{C 1}-P_{C 2}\right)}
$$

This definition is convenient experimentally since the upstream dynamic pressure is always known. The second coefficient, used for all loading measurements at offdesign incidence presented in Chapter 6 , is normalized by the outlet dynamic pressure and can be calculated from:

$$
C_{P l Q e}=\frac{P_{0 i}^{\prime}-P_{S l}}{P_{0 e}^{\prime}-P_{S e}^{\prime}}=C_{P l Q i}\left(\frac{U_{i}}{U_{e}}\right)^{2}
$$

This definition of pressure coefficient provides the correct visual impression of the changes in Zweifel coefficient for off-design incidence measurements, as described in Appendix B. 


\subsection{Data Reduction for Periodically-Unsteady Flow Measurements}

\subsubsection{Ensemble Averaging}

The ensemble mean is found when a certain quantity is averaged over a large number of experimental runs (cycles). Each cycle begins at a specified time after a trigger pulse. As a result of the ensemble averaging, a random fluctuations in the signal (noise) are eliminated from the data. Ensemble averaging was performed for the downstream hotwire data in the unsteady measurements. The trigger for data acquisition was obtained from the photo detector mounted on the spoke-wheel. The trigger was activated once-per-revolution of the wheel as described in Section 3.2.5.

The ensemble mean of quantity $\mathrm{X}$ at time $\mathrm{t}$ is defined as:

$$
\widetilde{X(t)}=\frac{1}{N} \sum_{i=1}^{N} X_{i}(t)
$$

where $\mathrm{N}$ is the total number of cycles and $\mathrm{t}$ is the time relative to the trigger signal. For the current measurements the resultant velocity at the exit plane, $U_{e}$, was measured using 256 cycles and for the passing of the same three consecutive bars. This procedure will be discussed further in the next section.

\subsubsection{Single Hotwire Measurements}

The hotwire measurements were performed at the traversing plane located $0.4 C_{x}$ downstream of the trailing edge. The probe traverse consisted of 100 points covering 
1.5 blade pitches ( 0.75 pitch on either side of the projection of trailing edge metal angle of the middle blade). Similar to the steady measurements, the points were spaced about $1.5 \%$ of the pitch. Prior to performing the unsteady tests, the wind tunnel operating point and the uniformity and periodicity of the flow were set according to the experimental procedure defined in Sections 4.2.1 and 4.2.3, respectively.

Figure 4.5 shows the ensemble-averaged velocity distributions at three different times normalized by the wake passing period, $\mathrm{T}$, corresponding to the beginning of the wake passing period $(t / T=0.0)$, the mid-period $(t / T=0.5)$ and the end of the period $(t / T=1.0)$. The velocity distribution obtained from steady measurements is shown as a solid line. As can be seen, the velocity profiles at the beginning and the end of the period agree closely, as expected. The velocity distribution data for each

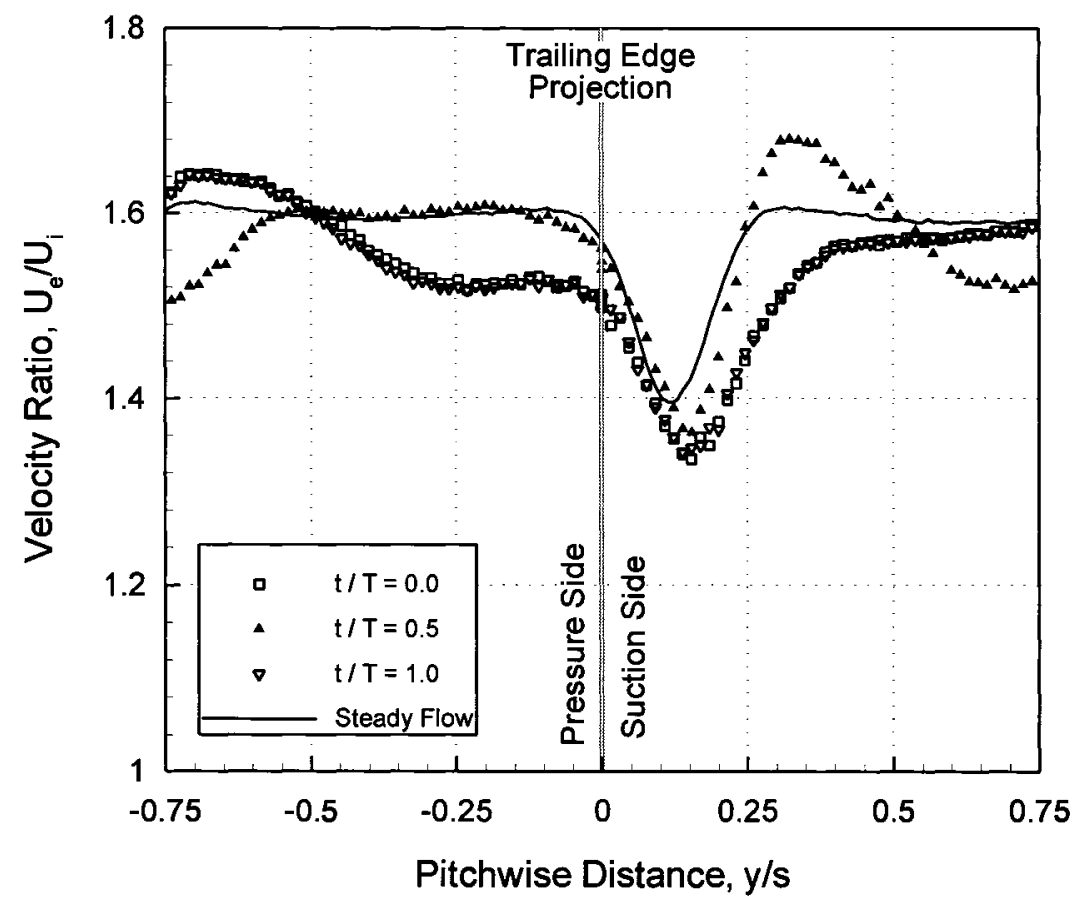

Figure 4.5: Typical Exit Velocity Profiles for PAKD-F 
value of $t / T$ was further reduced to calculate the mixed-out profile losses at those instances, as will be described in Section 4.3.4.

During measurements, the test section temperature $(T)$ was typically slightly different from the temperature during the hotwire calibration $\left(T_{\text {calib }}\right)$. To take into account the ambient temperature drift the temperature correction of Bearman (1971) was applied:

$$
\left(\frac{T_{h w}-T_{c a l i b}}{T_{h w}-T}\right) E_{T O B}^{2}=A+B U^{n}
$$

where $T_{h w}$ is the temperature of the hotwire and $E_{T O B}$ is the top-of-the-bridge voltage. Calibration coefficients $A, B$ and $n$ and the procedure used to obtain them were discussed in Section 3.5.4.

\subsubsection{Calculation of Bar Loss}

The measurements at the cascade outlet provide the total loss, $Y_{\text {total }}$, which includes both the losses due to the bars and those due to the airfoil. In order to calculate the airfoil losses, an estimate of the bar losses is thus needed.

The bar losses are difficult to measure experimentally. Conventional pressure probes and pressure transducers have too slow a response to allow the unsteady total pressure to be measured directly. Due to the length of pneumatic tubing, pressure transducer and pressure probe response, these measurements would have a significant time delay and signal attenuation. Therefore, the bar losses were estimated based on an assumed drag coefficient for the bars. From a control volume analysis using the continuity and momentum equations, the loss coefficient for the bars was obtained 
(Mahallati, 2003):

$$
Y_{b}=\frac{\Delta P_{0}}{\frac{1}{2} \rho U_{x i}^{2}}=C_{D} \frac{d}{s_{b}} Z\left[1-\frac{1}{4}\left(C_{D} \frac{d}{s_{b}} Z\right)\left(Z^{2}-1\right)+\left(Z^{2}-1\right)\right]
$$

where $C_{D}$ is the drag coefficient for the bar, equal to approximately 1.05 for the range of velocities (Reynolds numbers) encountered in the current measurements (Schlichting and Gersten, 2000), $d$ is the bar diameter and the bar pitch is $s_{b}$. Parameter $Z$ is calculated from:

$$
Z=\sqrt{1+\tan \alpha_{i}^{2}+\frac{2 \tan \alpha_{i}}{\phi}+\frac{1}{\phi}}
$$

where $\phi=U_{x i} / U_{b}$ is the flow coefficient, $U_{x i}$ is the inlet axial velocity and $U_{b}$ is the bar velocity. The significance of the flow coefficient will be addressed in Chapter 7 .

\subsubsection{Mixed-out Profile Loss}

The hotwire measurements described in Section 4.3.2 were used to calculate the resultant velocity at the exit plane, $U_{e}$, as a function of both time and space. As opposed to the steady measurements with the three-hole pressure probe, the single hotwire cannot provide information about the flow angle and static (or total) pressure. In order to calculate the profile losses, the following method developed by Mahallati (2003) was adopted. First, two assumptions were made:

- The outlet flow angle, $\alpha_{e}$, as a function of time, was estimated based on the geometric position of the minimum velocity relative to the blade trailing edge, as can be seen from Figure 4.6. It was assumed that there is no spatial variation in the outlet flow angle. Based on the steady measurements, this assumption 


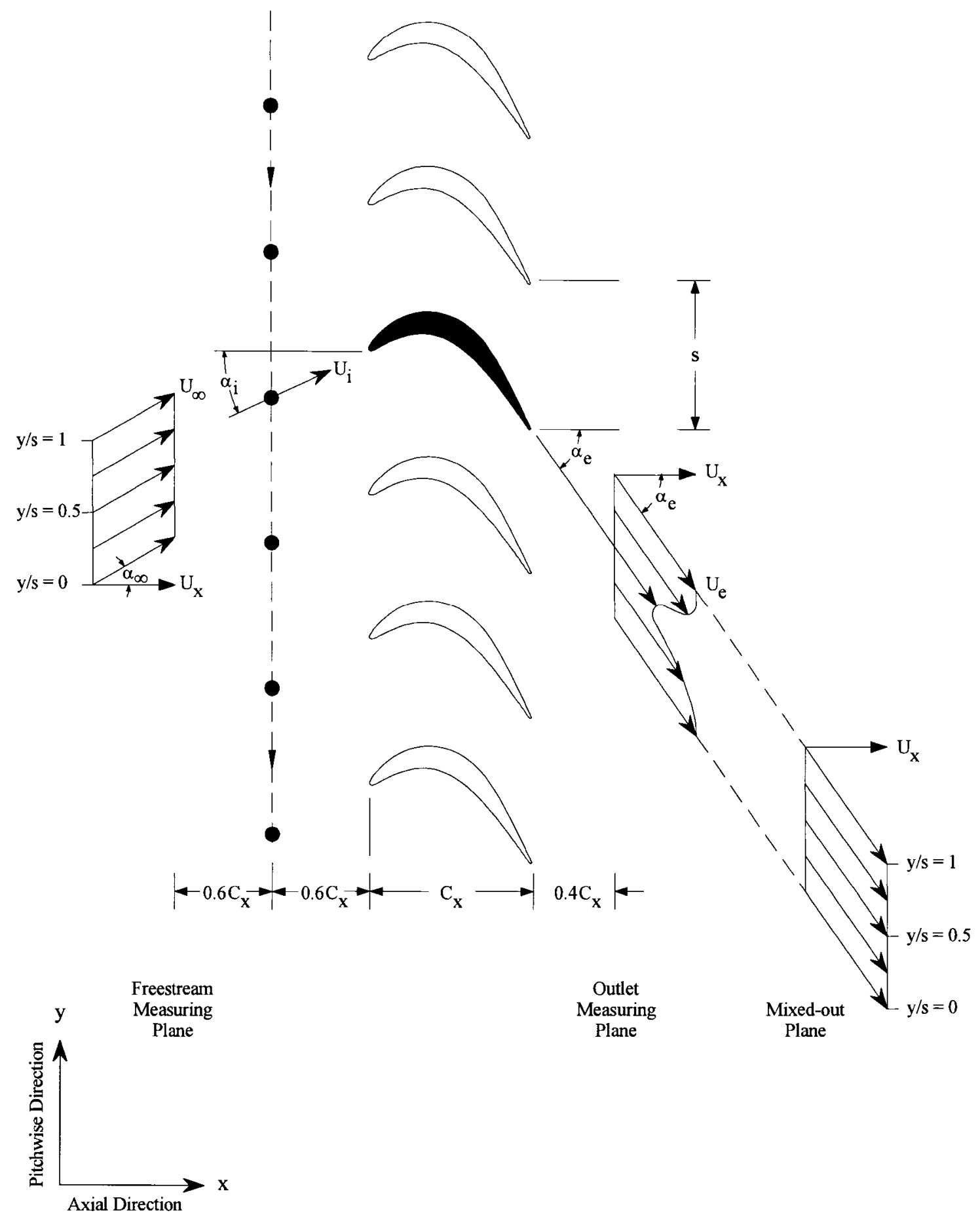

Figure 4.6: Measurement Planes and Nomenclature for Unsteady Flow (modified from Mahallati, 2003) 
appears to be valid, since spatial variations in the outlet flow angle are relatively small for non-stalled cases (when the flow is attached at the trailing edge).

- The outlet static pressure coefficient, $C_{P S e}$, was assumed to be constant in both time and space. The $C_{P S e}$ was estimated based on the time-averaged outlet measurements obtained with the three-hole probe at $0.4 C_{x}$ downstream of the trailing edge. This assumption is harder to justify since the observed uncertainty in the outlet static pressure has a greater impact on the profile losses than the uncertainty in the inferred outlet flow angle. The present author believes that in addition to the bar loss estimate, this is the main source of the experimental uncertainty in the unsteady measurements.

Data reduction for the unsteady losses was performed in the following way. Using the assumption of constant $\alpha_{e}$ and Eq. 4.14, the velocity ratio squared can be obtained from:

$$
\left(\frac{U_{m}}{U_{\infty}}\right)^{2}=\left[\cos \alpha_{e} \int_{0}^{1}\left(\frac{U_{e}}{U_{\infty}}\right) d(y / s)\right]^{2}+\left[\frac{\sin \left(2 \alpha_{e}\right) \int_{0}^{1}\left(\frac{U_{e}}{U_{\infty}}\right)^{2} d(y / s)}{2 \cos \alpha_{e} \int_{0}^{1}\left(\frac{U_{e}}{U_{\infty}}\right) d(y / s)}\right]^{2}
$$

where $U_{m}$ is the mixed-out outlet velocity, $U_{\infty}$ and $U_{i}$ are the inlet velocities upstream and downstream of the bars, respectively, as shown in Figure 4.6. The total pressure loss, including both the airfoil loss and the bar loss can be expressed using Eq. 4.13 as:

$$
Y_{\text {total }}=\frac{1-C_{P S e}+2\left(I_{1}^{2}-I_{2}\right)}{\left(\frac{U_{m}}{U_{\infty}}\right)^{2}}-1
$$


where $C_{P S e}$ is, as previously mentioned, the time-averaged outlet static pressure coefficient and $I_{1}$ and $I_{2}$ are the integrals defined in Eq. 4.15 and 4.16 in Section 4.2.5. Subtracting the bar losses from the total loss, the mixed-out profile loss due to the airfoil can be obtained:

$$
Y_{m}=Y_{\text {total }}-Y_{b a r}\left(\cos \left(\alpha_{\infty}\right) \frac{U_{\infty}}{U_{m}}\right)^{2}=\frac{1-C_{P S e}+2\left(I_{1}^{2}-I_{2}\right)-Y_{b} \cos ^{2}\left(\alpha_{\infty}\right)}{\left(\frac{U_{m}}{U_{\infty}}\right)^{2}}-1
$$

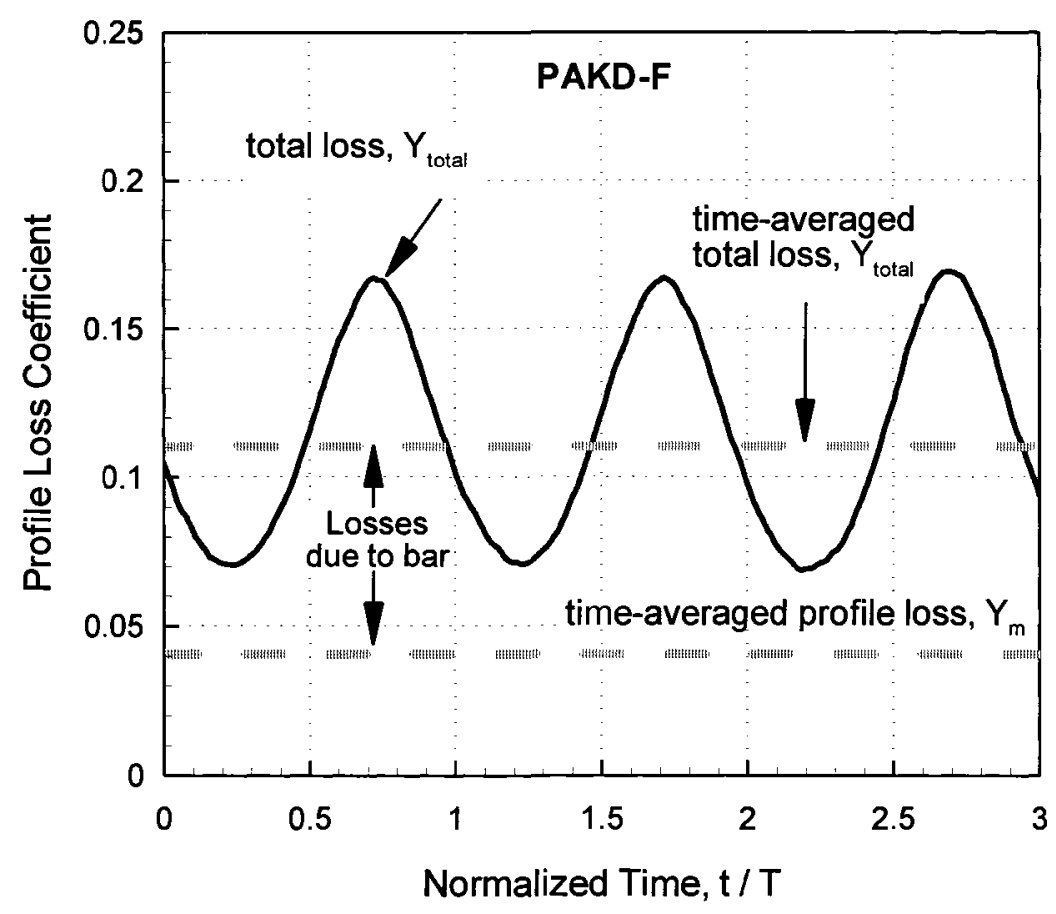

Figure 4.7: The losses for a Typical Unsteady Case

Figure 4.7 shows the total loss, $Y_{\text {total }}$, as a function of time, $t$, plotted over three wake passing periods, $T$. It can be seen that the total losses measured at the cascade outlet vary periodically. The dashed lines correspond to the time-averaged total and 
profile losses.

\subsection{Uncertainty Analysis}

Experimental errors can never be completely eliminated, although they can be minimized by careful design of an experiment, regular calibration of instrumentation etc. In order to discuss any experimental data, an estimate of the uncertainty is also required. The following is a brief description of Single-Sample Uncertainty Analysis described by Moffat (1982). This was the method used to estimate the uncertainties in the present work.

The uncertainty of a measured value, $x$, can be written as:

$$
\delta x= \pm 2 \sigma
$$

where $\sigma$ is the standard deviation in data. As long as the scatter in data is normally distributed (Gaussian distribution), the $\delta x$ represents the uncertainty with $95 \%$ confidence level. There are different sources of uncertainties coming from equipment inaccuracies and errors which arise from repeated measurement of the same quantity (repeatability or random errors). The combined error is influenced by all individual sources of error.

$$
X=f\left(x_{1}, x_{2}, \ldots, x_{N-1}, x_{N}\right)
$$

where $\mathrm{N}$ is the total number of fundamental parameters. If it is assumed that individual parameters are independent one from another, the uncertainty in $\mathrm{X}$ due to 
uncertainty in $x_{k}$ is then:

$$
\delta X_{k}=\left(\frac{\partial X}{\partial x_{k}}\right) \delta x_{k}
$$

The total, or combined, error is not a result of a simple addition of individual errors since they do not necessarily reach their maximum values at the same time. A more appropriate method is to calculate the Root-Mean-Square (RMS) average of the individual sources. Thus, the uncertainty due to all individual effects is given by:

$$
\delta X= \pm \sqrt{\sum_{k=1}^{N}\left(\delta X_{k}\right)^{2}}
$$

Due to limited number of calibrations performed on pressure transducers, threehole probe and hotwire, the estimated errors are based, in part, on repeated calibration by Benner (2003) and Mahallati (2003) over a number of years. The values of uncertainty are presented where appropriate either by using error bars on figures or by quoting the values in the respective sections. 


\section{Chapter 5}

\section{Performance for Steady Flow at Design Incidence}

\subsection{Introduction}

This chapter focuses on the steady measurements at the design incidence for all three airfoils. The measurements were performed at the midspan for two different free stream turbulence intensities (1.5\% and $4 \%$ ). The PAKB and PAKD-A blades have been extensively tested during the last couple of years; in addition to the present author, Mahallati (2003), McAuliffe (2003) and Dai (2004) examined the midspan behavior and Zorić (2006) was concurrently investigating the secondary flows in the absence of tip leakage. Since the present work concentrates on Reynolds number sensitivity of the PAKD-F airfoil, some of the previously investigated test cases have been included in this chapter in order to compare the performance of the three airfoils. Table 5.1 summarizes test cases that will be discussed and the origins of the data. 
Table 5.1: Summary of Test Cases Presented

\begin{tabular}{|c|c|c|c|c|c|c|}
\hline & \multicolumn{2}{|c|}{ PAKB } & \multicolumn{2}{|c|}{ PAKD-A } & \multicolumn{2}{|c|}{ PAKD-F } \\
\hline $\mathbf{R e}_{\mathrm{i}} /$ FSTI & $1.5 \%$ & $4 \%$ & $1.5 \%$ & $4 \%$ & $1.5 \%$ & $4 \%$ \\
\hline 150,000 & $\mathbf{L} / \mathbf{P}^{1}$ & $L / P^{2}$ & $L / P^{3}$ & $\mathrm{~L} / \mathrm{P}^{3}$ & $\mathbf{L} / \mathbf{P}$ & $L / P$ \\
\hline 100,000 & $L / \mathbf{P}^{1}$ & $\mathrm{P}^{2}$ & $L / P^{3}$ & $P^{3}$ & $L / P$ & \\
\hline 80,000 & $L / P$ & $\mathbf{P}$ & $L / P$ & $\mathbf{P}$ & $\mathbf{L} / \mathbf{P}$ & $\mathbf{P}$ \\
\hline 75,000 & & & $L / P^{3}$ & & & \\
\hline 65,000 & & & $\mathrm{~L} / \mathrm{P}^{3}$ & & & \\
\hline 50,000 & $\mathrm{~L} / \mathrm{P}^{1}$ & $P^{2}$ & & $P^{3}$ & $\mathbf{L} / \mathbf{P}$ & \\
\hline 40,000 & & & & $L / \mathbf{P}^{3}$ & $L$ & \\
\hline 35,000 & & & & $L / P^{3}$ & $\mathbf{L}$ & \\
\hline 30,000 & & & & & $\mathbf{L}$ & \\
\hline 25,000 & $\mathrm{~L} / \mathrm{P}^{1}$ & $L / \mathbf{P}^{2}$ & & & $\mathrm{~L} / \mathrm{P}$ & $L / P$ \\
\hline
\end{tabular}

L: Loading, P: Profile Loss, ${ }^{1}$ Mahallati (2003), ${ }^{2}$ McAuliffe (2003) and ${ }^{3}$ Dai (2004)

The Reynolds number is based on the cascade inlet velocity and the axial chord.

This chapter will begin with a short description of the aerodynamic loading of the three airfoils at design conditions. Section 5.3 will present aerodynamic measurements for PAKD-F covering a wide range of Reynolds numbers. The current measurements for PAKD-F will be compared in Section 5.4 with some of the previous measurements for PAKB and PAKD-A. Finally, the results will be summarized in the last section.

\subsection{Comparison of Loadings at Design Conditions}

Static pressure distributions in terms of $C_{P l}$ (as defined in Equation 4.18) are plotted against normalized axial position $\left(x / C_{x}\right)$ for all three airfoils in Figure 5.1. The airfoils are compared at "design conditions" (as chosen for the purpose of this 
study): zero flow incidence, $R e_{i}$ of 80,000 and FSTI of $1.5 \%$.

The tangential force produced by an airfoil is directly related to the pressure differential between the suction and the pressure sides of the airfoil; the larger the area enclosed by the two curves, the larger the tangential force. As already discussed in Section 3.2.3, both PAKD airfoils are subjected to approximately $25 \%$ higher loading than PAKB. This can clearly be seen in Figure 5.1. On the suction side of the airfoil (the upper curve) the flow accelerates until the suction peak, the point of maximum

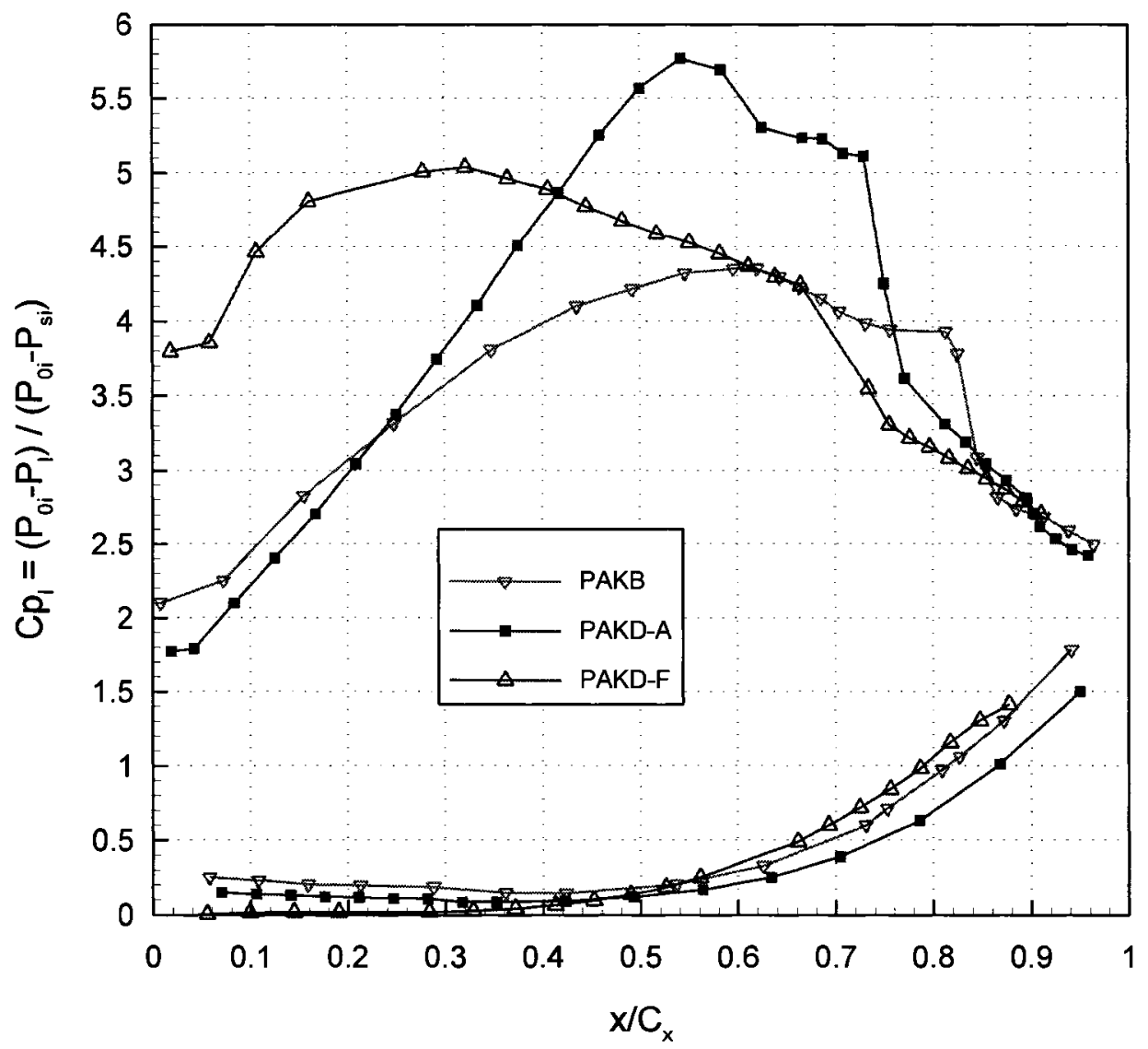

Figure 5.1: Comparison of Loadings at Design Conditions

$$
\left(R e_{i}=80,000, \mathrm{FSTI}=1.5 \% \text { and Design Incidence }\right)
$$


velocity and minimum pressure. The location of the suction peak for the PAKD-F airfoil is at approximately $0.32 C_{x}$, while PAKB and PAKD-A have the suction peaks at 0.61 and $0.55 C_{x}$, respectively. Thus, PAKD-F is front loaded (the tangential force is predominantly acting on the forward portion of the blade), while PAKB and PAKDA are more aft loaded. As mentioned earlier, the differences in loading distributions between PAKD-A and PAKD-F were achieved mainly by changing the stagger angle.

As the flow passes the suction peak, it encounters an adverse pressure gradient and begins to decelerate, as identified by the reduction in $C_{P l}$. For both PAKB and PAKD-A, shortly after the suction peak the flow separates and forms a laminar separation bubble. This can be identified by the plateau in the pressure distribution. Naturally, one of the necessary conditions for the existence of a laminar separation bubble is that the flow remains laminar up to the point where it encounters the stronger adverse pressure gradient and then separates, as discussed in Section 2.4.2. Both PAKB and PAKD-A experience a turbulent reattachment of the flow at 0.16 and $0.14 C_{x}$ downstream of the separation point. As will be discussed in next section, it is the author's belief that PAKD-F does not have a laminar separation bubble under these conditions. Instead the laminar boundary layer appears to undergo bypass type transition before encountering the stronger adverse pressure gradient at about 0.67 $C_{x}$.

The next section will present the Reynolds number sensitivity of PAKD-F, while the aerodynamic behavior of all three airfoils under will be further compared in Section 5.4. 


\subsection{Reynolds Number Sensitivity of PAKD-F}

\subsubsection{Loading Distributions at FSTI of $1.5 \%$}

The loadings data for the PAKD-F airfoil are plotted in Figure 5.2. The differences in loadings for the Reynolds numbers ranging from 50,000 up to 150,000 are not significant and can be attributed to the experimental uncertainty, which was estimated to range between $\partial C_{P l}= \pm 0.03$ and $\partial C_{P l}= \pm 0.15$ for Reynolds numbers between 150,000 and 25,000 , respectively.

The loading curves shown in Figure 5.2 show a sudden drop in $C_{P l}$ between 0.67 to $0.74 C_{x}$ for Reynolds numbers between 50,000 and 150,000 . Sudden drops in $C_{P l}$ distributions are often attributed to the existence of separation bubbles. As discussed in Section 2.4.2, a separation bubble can be identified through a plateau in the pressure distribution followed by a pressure recovery. Unfortunately, it was not possible to instrument the blade with static pressure taps in this region due to the interference with the metal rod used to mount the blade on the cascade base plate.

The author is of the opinion that the source of the $C_{P l}$ drop is not the separation bubble, but the effect of blade surface curvature distribution. Korakianitis and Papagiannidis (1993) reported that small discontinuities in airfoil curvature can result in spikes or humps in surface pressure and velocity distributions. To investigate this, a computational simulation using a commercial CFD package, CFX5, was performed. The shear stress transport (SST) turbulence model and default settings were used. Since the version of CFX used does not have a transition model, a fully turbulent flow, from the leading to the trailing edge, was assumed. The boundary conditions were set to match the midspan flow around the middle blade in the cascade. 


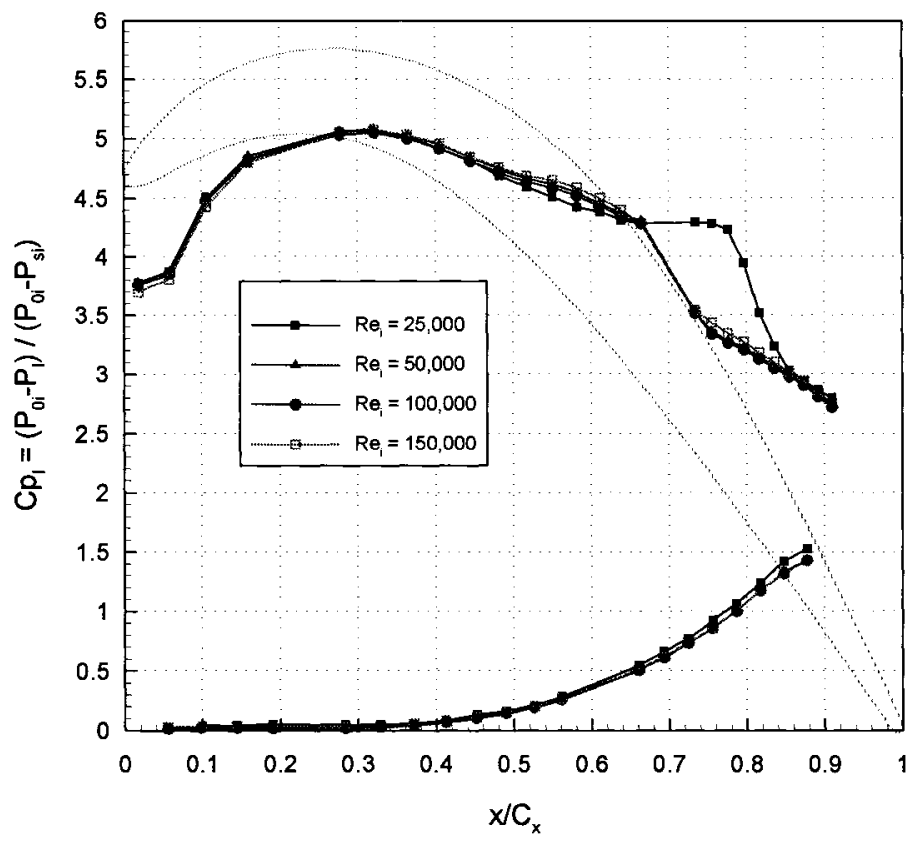

Figure 5.2: Loadings for PAKD-F at FSTI of $1.5 \%$

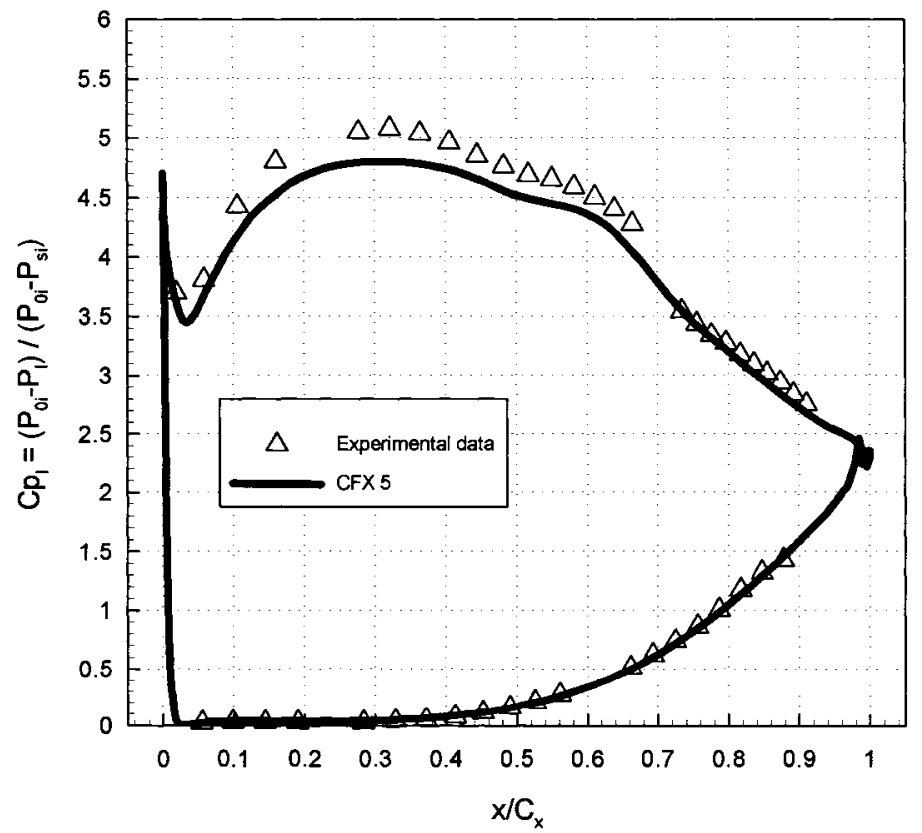

Figure 5.3: Comparison of Experimental and CFX 5 Computational Results (PAKD-F: 150,000, FSTI $=1.5 \%$ and Design Incidence) 
Since the numerical simulation was performed with the fully turbulent boundary layer, there are no separation bubbles present on the blade surface. Thus, the pressure distribution obtained numerically is due to the blade geometry only. The experimental and numerical results are compared in Figure 5.3. They imply that sudden drop in the $C_{P l}$ obtained experimentally is due to the discontinuity in curvature distribution. However, these conclusions should be supported with either flow visualization or hot film measurements.

On the other hand, at a Reynolds number of 25,000 , a separation bubble clearly is present. It extends from about 0.65 to $0.85 C_{x}$. The pressure distribution just upstream of the separation point seems to be only slightly affected by its presence. Thus the bubble is of the short type (Gaster, 1967). In order to examine the development of the bubble more closely, additional loading measurements were taken in the range of $R e_{i}$ varying from 25,000 to 50,000 . These results are presented in Figure 5.4.

As the Reynolds number increases from 25,000 to 50,000 , the flow separates at $0.67 C_{x}$, but the length of the resulting separation bubble decreases progressively as summarized in Table 5.2. As discussed in Section 2.5, the transition point (generally) moves upstream as the Reynolds number is increased (Mayle, 1991). For Reynolds numbers higher than about 45,000 , it is believed that the boundary layer undergoes transition, perhaps via bypass mode, before the point where the laminar boundary layer would separate $\left(0.67 C_{x}\right)$. Since the turbulent boundary layer is more resistant to the separation, the flow stays fully attached all the way to the trailing edge, and consequently there is no separation bubble present on the suction surface of the PAKD-F airfoil. 


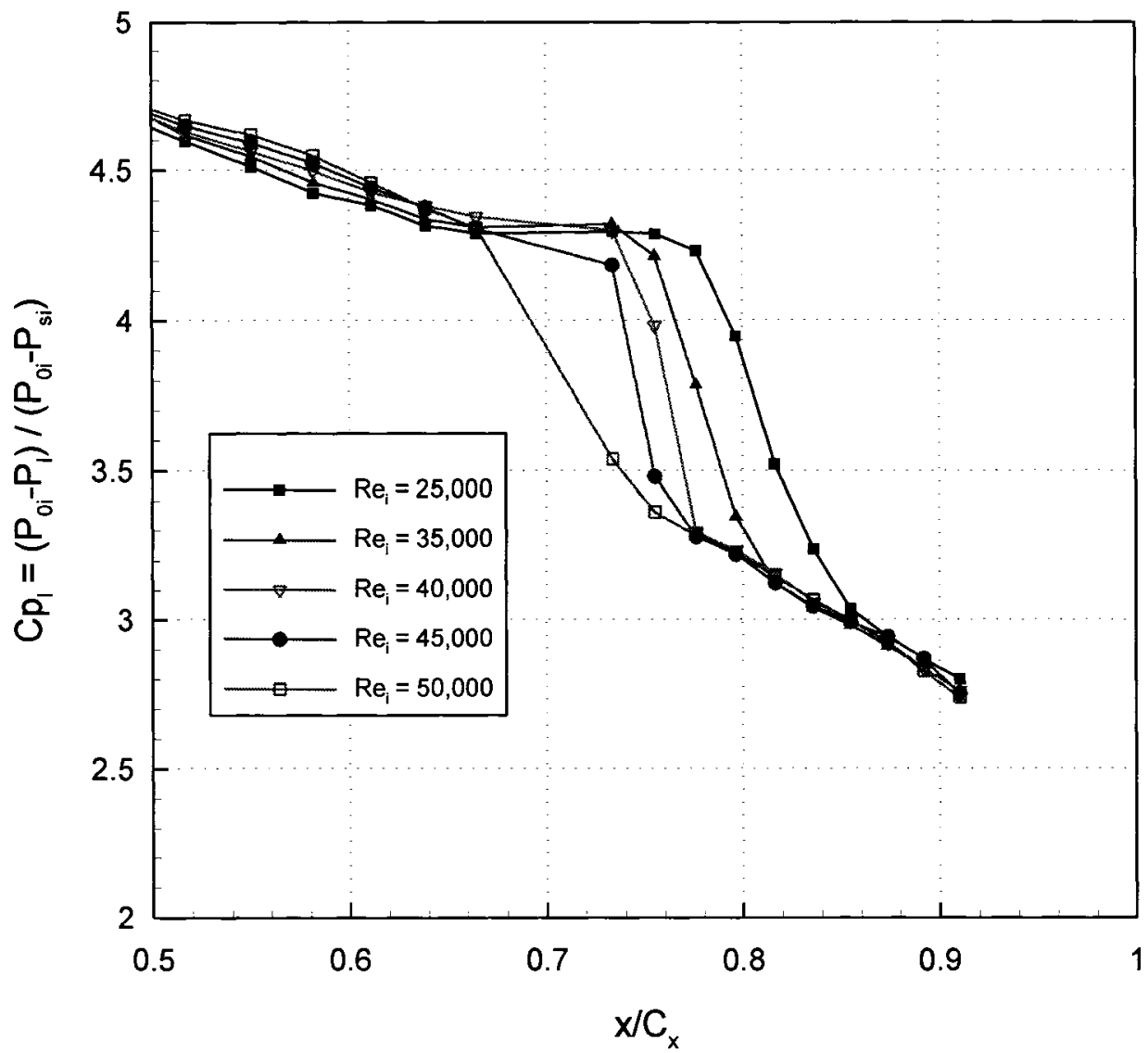

Figure 5.4: Bubble Growth on the Suction Side of PAKD-F at FSTI of $\mathbf{1 . 5 \%}$

Table 5.2: Summary of Separation and Reattachment Points

\begin{tabular}{|c|c|c|}
\hline \multirow{2}{*}{$\mathbf{R e}_{\mathbf{i}}$} & Separation point & Reattachment point \\
\cline { 2 - 3 } & \multicolumn{2}{|c|}{$\% \mathbf{C}_{\mathbf{X}}$} \\
\hline 50,000 & None & None \\
\hline 45,000 & 67 & 76 \\
\hline 40,000 & 67 & 78 \\
\hline 35,000 & 67 & 80 \\
\hline 25,000 & 67 & 85 \\
\hline
\end{tabular}


The experimental results shown in Figure 5.2 obtained from the first pressure tap on the suction surface of the PAKD-F airfoil indicate the existence of the velocity spike (overspeed) in this region. From the loading plot obtained numerically (shown in Figure 5.3), a significant velocity spike is clearly visible near the leading edge of the airfoil. Benner (2003) discussed the role of curvature distribution discontinuity in producing leading-edge velocity spikes which can be followed by small separation bubbles. He argued that immediately after the spike the strong adverse pressure gradient can cause the boundary layer, which is still laminar at this point, to separate. Shortly after, the boundary layer reattaches, possibly as a turbulent one. However, since there is strong acceleration immediately after the reattachment it is likely that the turbulent boundary will subsequently undergo reverse transition (relaminarization). As discussed in Section 2.4.1, Mayle (1991) suggested that the relaminarization occurs when the acceleration parameter exceeds the value of $3 \times 10^{6}$. In the present case the maximum value of the acceleration parameter is about $2.4 \times 10^{7}$, which is significantly above the threshold, implying that the condition necessary for the relaminarization exists.

\subsubsection{Influence of Higher FSTI (4\%) on Loading}

Figure 5.5 compares the blade loadings for PAKD-F at FSTI $1.5 \%$ and $4 \%$. At a Reynolds number of 150,000 , there seems to be no separation bubble for both levels of FSTI, and the loadings are very similar.

At $R e_{i}$ of 25,000 , for both FSTI cases, the flow separates at the same point. The reattachment, however, occurs earlier for the higher level of FSTI. The reattachment points are estimated to be 0.83 and $0.85 C_{x}$ for $4 \%$ and $1.5 \%$ FSTI, respectively. As would be expected, the higher FSTI appears to promote earlier transition in the free 


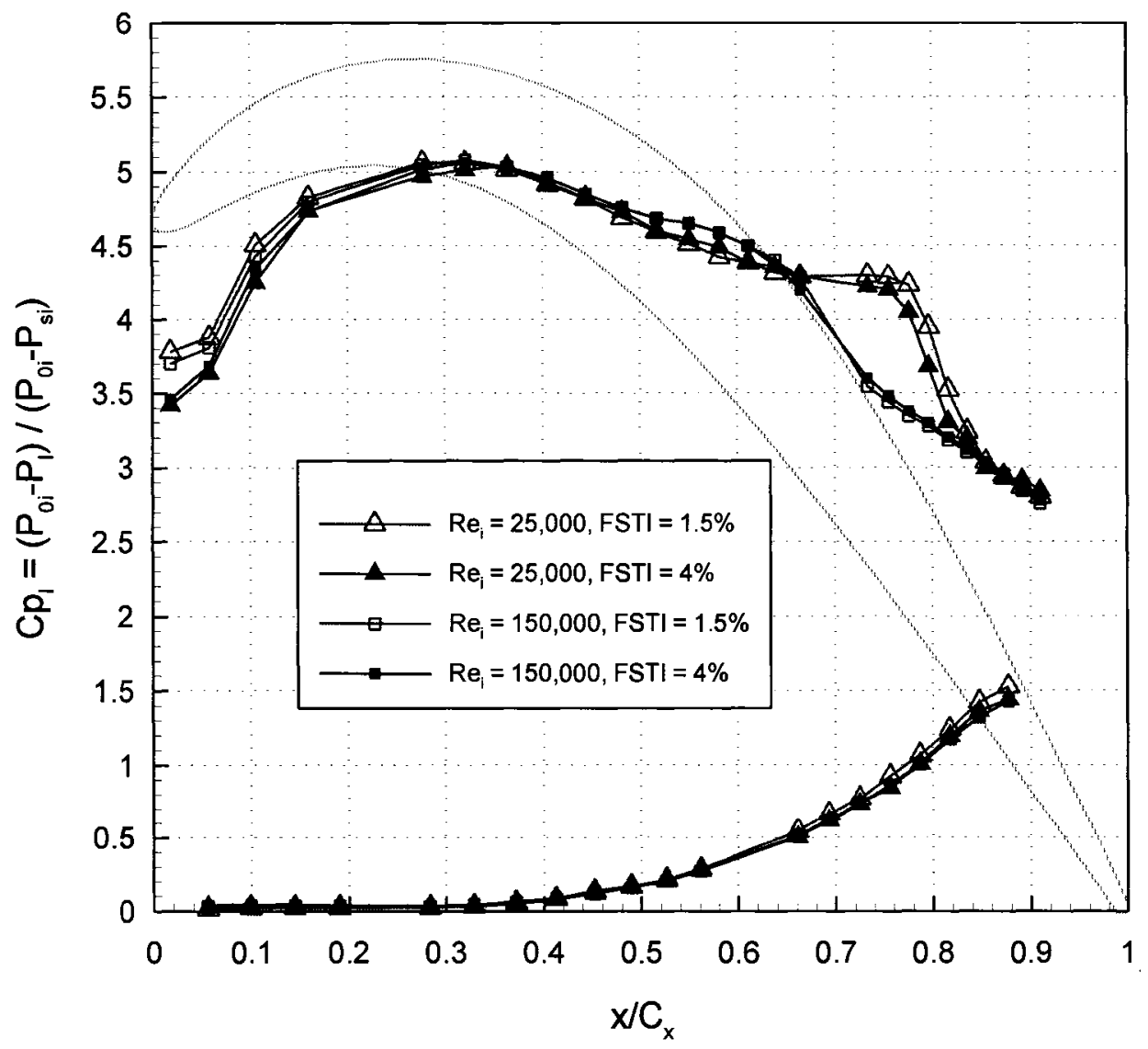

Figure 5.5: Loadings for PAKD-F at FSTI of $1.5 \%$ and $4 \%$

shear layer, and hence earlier reattachment.

The first pressure tap on the suction side shows a slightly larger values of $C_{P l}$ for the cases tested at FSTI of $1.5 \%$. This might be related to a perhaps short separation bubble near the leading edge that was discussed in the previous section. 


\subsubsection{Axial Velocity Ratios at Midspan}

Figure 5.6 presents the values of Axial Velocity Ratio (AVR) obtained as a function of Reynolds number for the PAKD-F airfoil. As discussed in Section 4.2.4, AVR of 1.0 is a necessary, but not sufficient condition, for two-dimensional flow at the midspan.

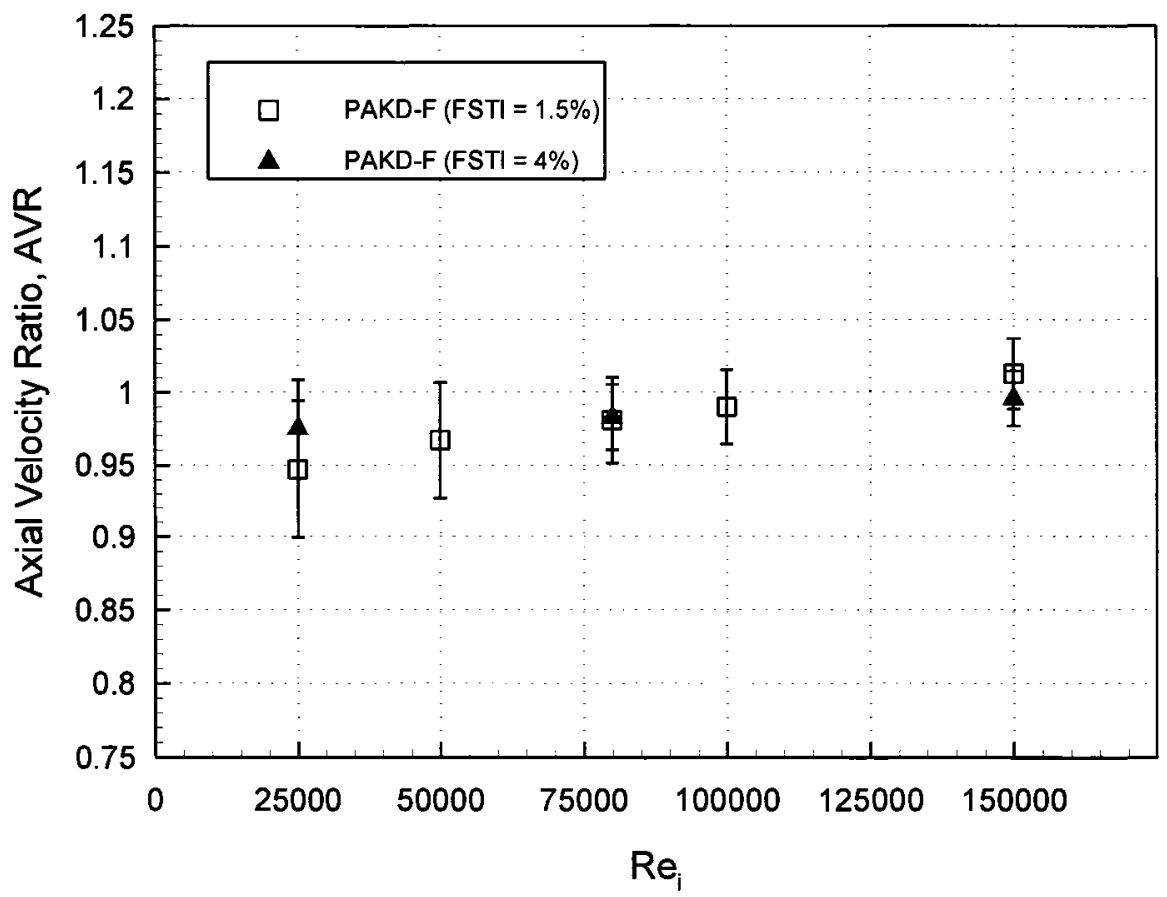

Figure 5.6: Axial Velocity Ratios for PAKD-F

A study by Rodger (1992) showed that the values of the AVR greater than 1.0 have smaller effect on the airfoil performance than values lower than 1.0. Values of AVR lower than 1.0 result in increased profile losses and outlet flow angles. Rodger observed an increase in losses of nearly $60 \%$ and an increase in outlet flow angle by about $1.5^{\circ}$ for AVR of 0.95 (compared to AVR of 1.0). Therefore, the losses and the flow angles at low Reynolds numbers that will be presented here may be higher than they would be if the AVR was 1.0. 
It should be also noted that the average uncertainty in AVR is estimated to be \pm 0.029 . It is mainly influenced by the uncertainty in the outlet flow angle. Similar values for uncertainty in AVR were reported by Mahallati (2003), McAuliffe (2003) and Benner (2003).

\subsubsection{Mixed-out Profile Losses}

The mixed-out profile loss coefficient, $Y_{m}$, for the present measurements is plotted in Figure 5.7. As can be seen, the loss increases with decreasing Reynolds number.

As mentioned earlier, for the range of $R e_{i}$ between 50,000 and 150,000 it is believed there is no laminar separation bubble and that transition occurs via bypass mode. As the Reynolds number decreases from 150,000, the transition point is expected to move downstream reducing the wetted area covered by the turbulent boundary layer. This would be expected to decrease the losses. However, at the same time the rate of dissipation in the laminar boundary layer may be significantly increasing as discussed in Section 2.5. This range of Reynolds numbers was classified by Denton (1993) as the transitional one, where the exact variation of losses depends on the details of the surface pressure distribution. The net result, in this case, is a gradual loss increase with decrease in Reynolds number.

As the laminar separation bubble appears at $R e_{i}$ of about 45,000 , the losses rise more rapidly. The separation bubble is expected to cause an increase in momentum thickness near the trailing edge, which would be reflected in a rise of the profile loss. The higher losses measured for Reynolds number of 25,000 at FSTI of $1.5 \%$ might also be associated with the low value of AVR, as mentioned in the previous section. The losses are generally lower for the higher FSTI. This is clearly caused by a reduction in 


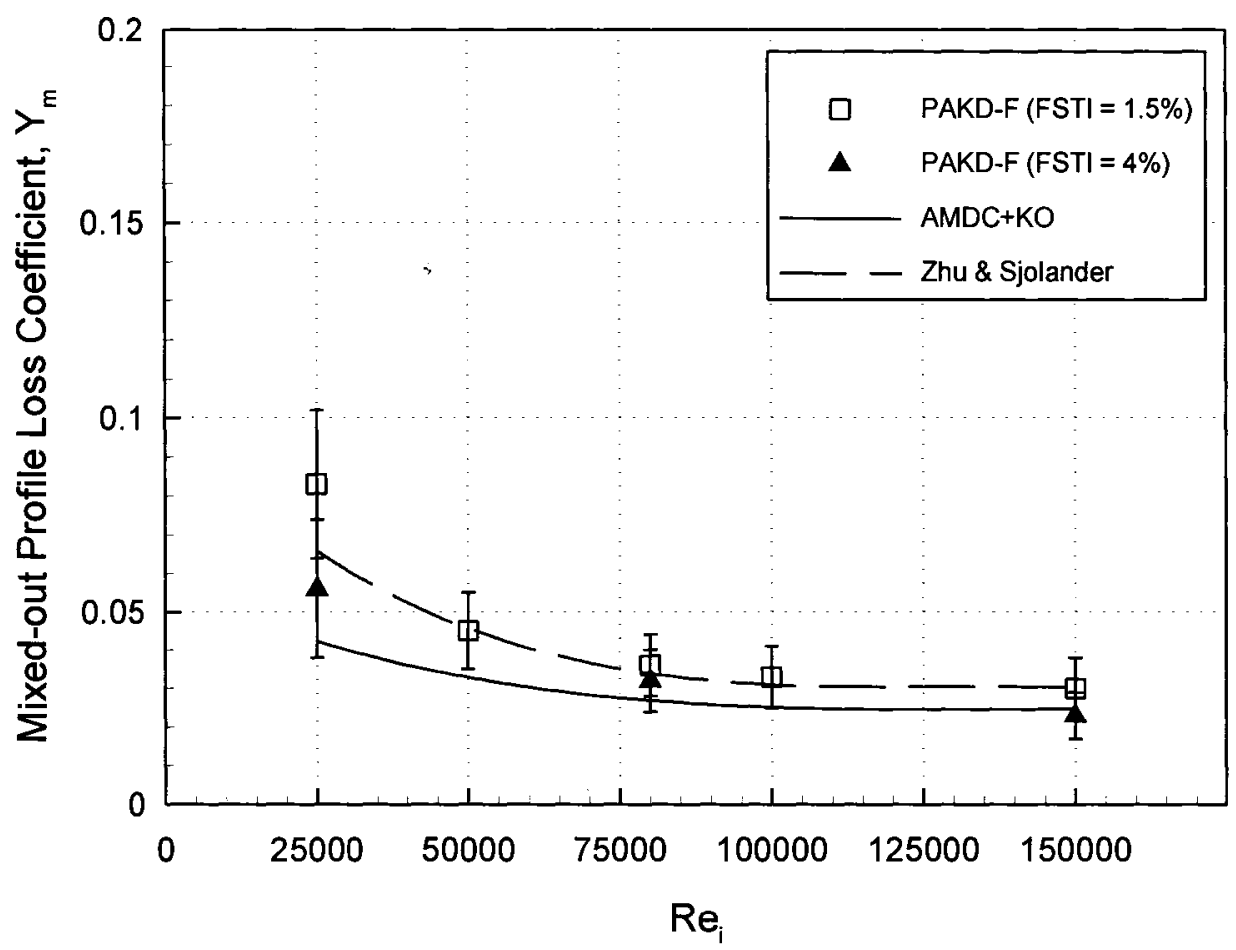

Figure 5.7: Mixed-out Profile Losses for PAKD-F

size of the separation bubble on the suction side of the airfoil for the lowest Reynolds number examined $(25,000)$. For the two higher Reynolds number cases, it is believed that a possible reduction in the leading edge separation bubble due to the higher FSTI might be responsible for the slightly lower losses.

At the preliminary stage of turbine design, it is very useful for a designer to predict the losses accurately. There are several correlations that are available for design point loss prediction, but the one by Kacker and Okapuu (1982) is widely used. Figure 5.7 shows that this correlation provides a satisfactory agreement at higher Reynolds numbers, but at the same time it significantly underestimates the losses at low Reynolds numbers. Recently, a modification to this correlation by Zhu and Sjolander (2005) was developed, partly using the present data, and it is seen to 
be in better agreement with the current measurements.

\subsubsection{Mixed-out Flow Angles}

The mixed-out outlet flow angles are shown in Figure 5.8. The outlet flow angle remains almost constant at about $59^{\circ}$ for $R e_{i}$ higher than 50,000 . With a decrease in Reynolds number to 25,000, the outlet flow angle and hence the flow turning increases slightly. As will be shown in Section 5.4.4, this trend is opposite of what was measured for PAKB and PAKD-A airfoils.

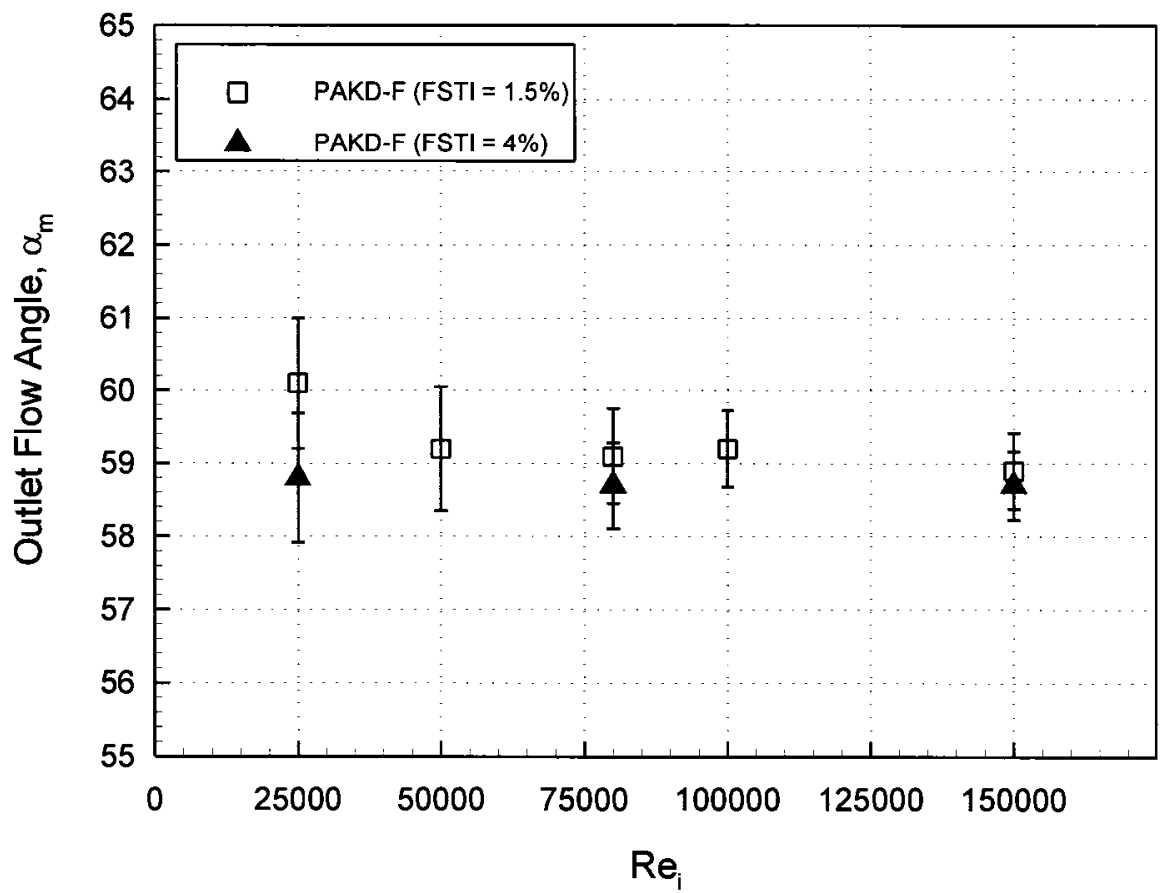

Figure 5.8: Mixed-out Outlet Flow Angles for PAKD-F

As previously mentioned, the results for the outlet flow angle are also influenced by AVR. Based on the observations of Rodger (1992), an increase in outlet flow angle by $1.5^{\circ}$ can occur for AVR of 0.95 compared to AVR of 1.0. This estimate seems 
consistent with the results obtained at FSTI of $1.5 \%$ and $4 \%$ and Reynolds number of 25,000 . The AVR values were about 0.95 and 0.98 , respectively, and from Figure 5.8 the outlet flow angles were seen to differ by about $1.3^{\circ}$.

\subsection{Aerodynamic Comparison of PAKB and PAKD- A with PAKD-F}

\subsubsection{Loading Distributions at FSTI (1.5\%)}

An overview of loading characteristics of each airfoil was provided in Section 5.2 where it was shown that PAKB and PAKD-A are aft-loaded and PAKD-F is a frontloaded airfoil. Thus, this section will focus mainly on the bubble behavior on the suction side of the three airfoils.

Figure 5.9 compares loading plots for PAKB (Mahallati, 2003), PAKD-A (Dai, 2004) and the present measurements for PAKD-F. All measurements shown in the figure were made at FSTI of $1.5 \%$. The figures in the right hand column show enlargements of the loading distributions on the suction side in the region of the separation bubble. In the case of PAKD-F, the enlargement includes the pressure distributions at additional values of the Reynolds number.

For PAKB, the flow undergoes an acceleration on the front portion of the suction surface, the region where the boundary layer is still laminar. Then shortly after the suction peak the flow separates at all Reynolds numbers investigated at approximately $0.74 C_{x}$. The length of of the separation bubble decreases with increasing $R e_{i}$. For the two high Reynolds number cases $(150,000$ and 100,000), the separation bubble 

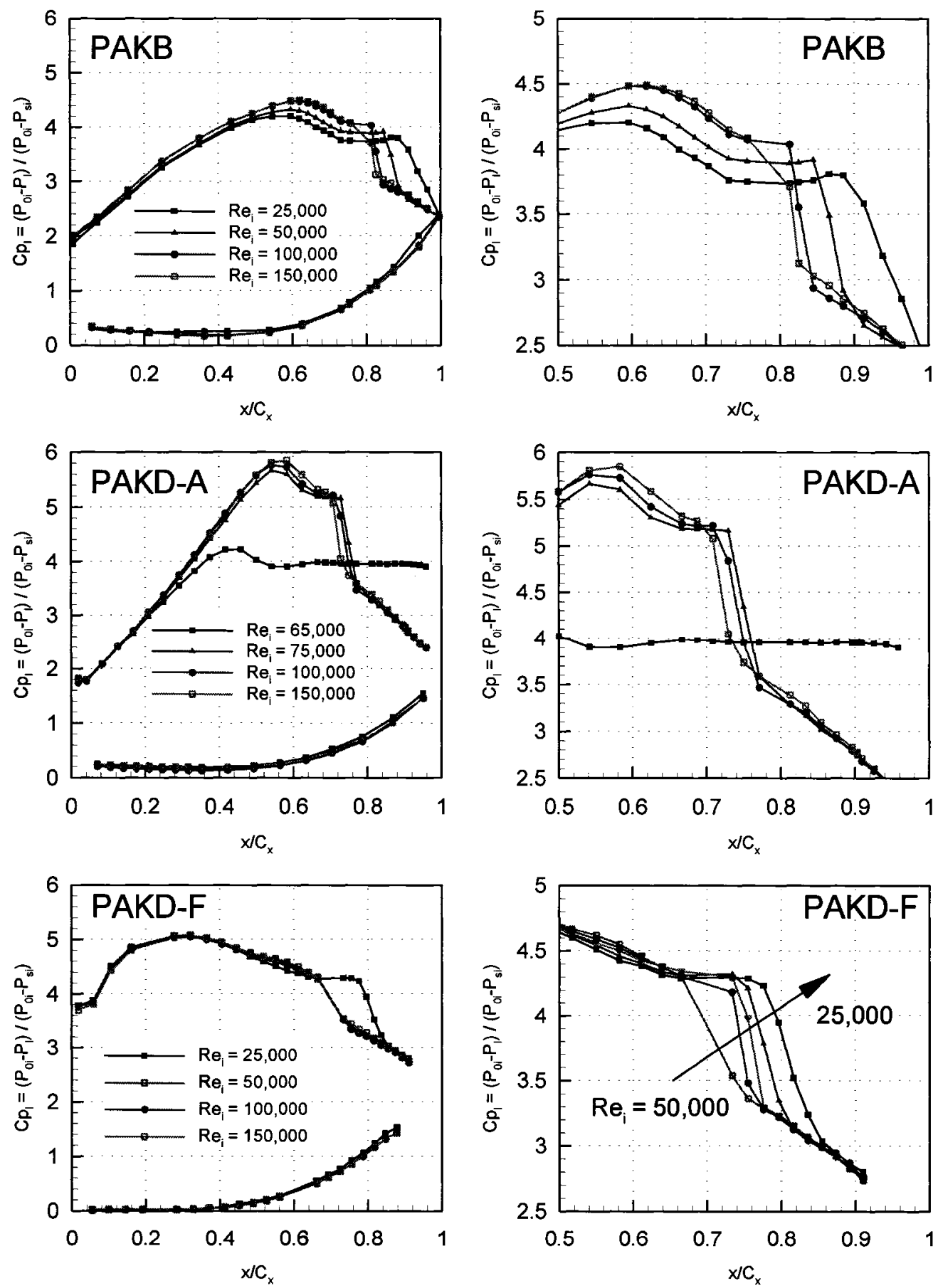

Figure 5.9: Comparison of Loadings at FSTI of $1.5 \%$

(PAKB data from Mahallati, 2003 and PAKD-A data from Dai, 2004) 
has very little influence on the pressure distribution upstream of the bubble (short type bubble), while for lower Reynolds numbers there is an appreciable change in the pressure distribution upstream of the bubble (long type bubble). At $R e_{i}$ of 25,000 the bubble reattaches just before the trailing edge. In all cases, the boundary layer reattaches as a turbulent one. This was verified by hotfilm measurements by Mahallati (2003). In addition, Mahallati has shown that the separation bubble fails to reattach for this Reynolds number at FSTI of $0.4 \%$, thus creating an open bubble and the blade stalls.

It can be concluded that the PAKD-F behavior is somewhat similar to that of PAKB, once a noticeable bubble starts developing on PAKD-F for $R e_{i}$ below 50,000. For both airfoils, any reduction in the Reynolds number leads to the growth in the bubble size. However, unlike PAKB, the bubble on PAKD-F seems to be of a short type with a minimal influence on the pressure distribution upstream of the separation point, even for the lowest Reynolds number examined.

A separation bubble is present on the suction side of PAKD-A for all cases investigated. At higher Reynolds numbers between 75,000 and 150,000, the bubble is of the short type, and its size increases slightly with reduction in Reynolds number. However, based on the measurements by Dai (2004), at a certain Reynolds number between 65,000 and 75,000 the separation bubble "bursts" abruptly creating an open bubble with a sharp increase in losses and decrease in the outlet flow angle. The present author encountered bursting at slightly higher $R e_{i}$ (between 75,000 and $80,000)$ and found the "bursting" process to be unsteady, as will be shown below.

In an attempt to examine whether in the appearance of the "bursting" of the bubble a hysteresis effect is present, numerous tests were performed by the present author for a range of $R e_{i}$, by either slowly increasing or decreasing $R e_{i}$. No hysteresis 

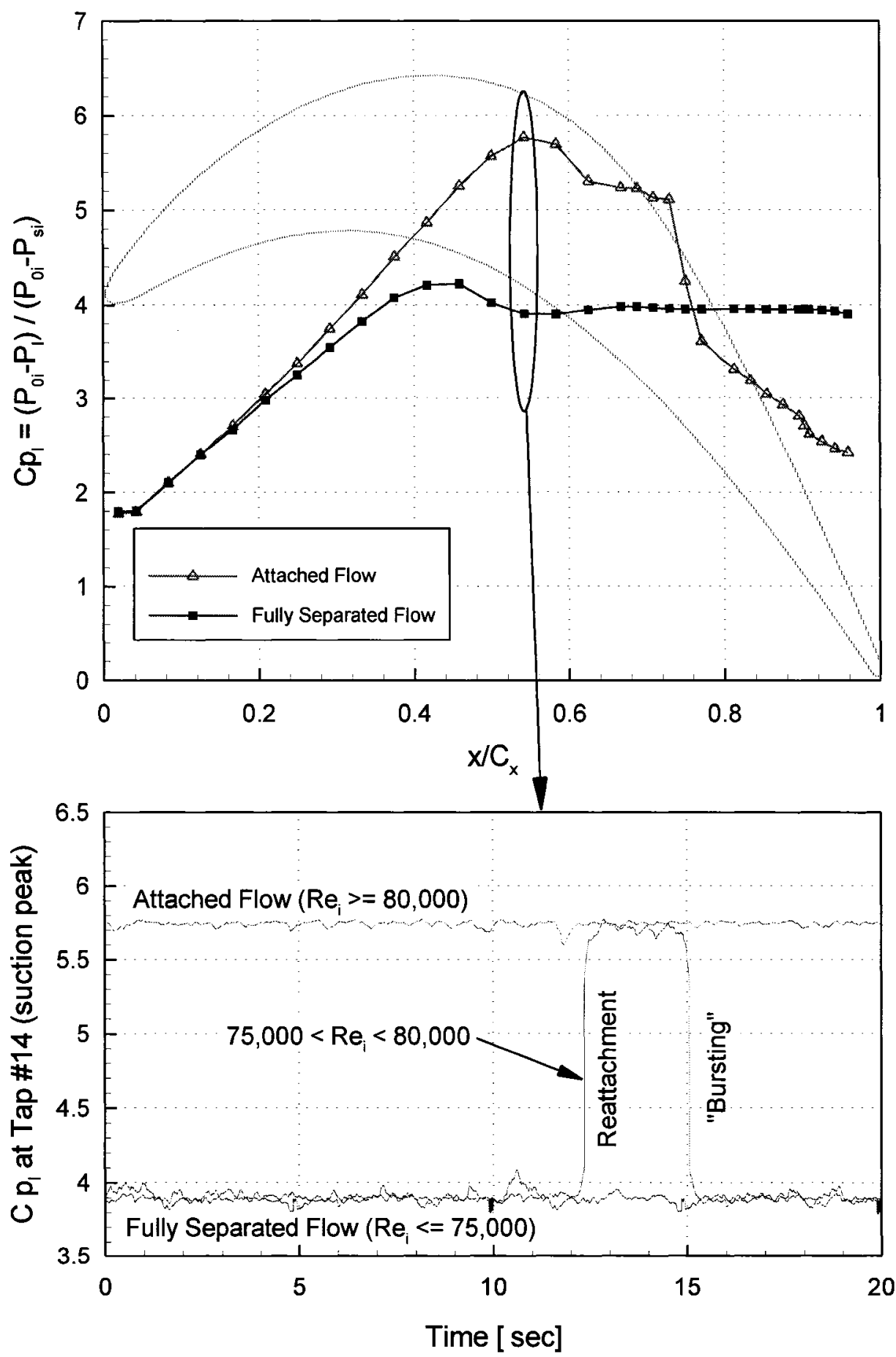

Figure 5.10: Bubble "Bursting" on PAKD-A at FSTI $=1.5 \%$ 
was noticed, but instead it was discovered that the bursting process is unsteady. There seems to exist a narrow range of $R e_{i}$ between 75,000 and 80,000 , at which the flow is unsteady and the blade periodically stalls. Shown in Figure 5.10 are the instantaneous values of $C_{P l}$ at the suction peak (tap number 14) for the following three cases: reattaching flow (typically $R e_{i}$ of 80,000 or higher), fully stalled (less than $75,000)$ and one of the periodically stalled cases. The data were taken for a period of 20 seconds. It can be seen that for the intermediate case, for the first 12 seconds the blade was fully stalled, but then suddenly the value of pressure coefficient at the suction peak jumped to its fully-attached value of approximately 5.75. After 3 seconds of attached flow, the bubble "burst" again and the flow became fully separated. The fraction of the time during which stall is present increases with a decrease in $R e_{i}$ until the bubble stops reattaching altogether and the flow becomes fully separated. It should be noted that the reattachments and bursting appear to be random and dependent on $R e_{i}$ only for a fixed value of FSTI and incidence.

Mayle (1991) suggested that small changes in either Reynolds number or angle of attack (incidence) can cause the bubble to "burst" from short to long, leading to a dramatic loss in lift and possibly a stall. In addition, he concluded that the difference between the long and the short bubble is not the length of transition, but mainly the length of the unstable laminar shear layer. The bubbles identified on PAKD-A are clearly of the short type with minimum effect on the overall pressure distribution, and the "bursting" observed appears to be the same phenomenon that Mayle described.

A recent investigation by Houtermans et al. (2004) on a high-lift front-loaded airfoil clearly identified short and long bubble types. Houtermans et al. showed that the change of the bubble state from short to long is not gradual. Furthermore, they showed that there is a certain Reynolds number when the bubble can periodically 
change its state (i.e. oscillate between two states).

For PAKD-A, the bubble appears to be either of the short type or the flow is fully separated (stall), without an intermediate long-bubble case. Walker (1993) proposed that whether a long bubble is possible or not, depends on the airfoil geometry (pressure distribution) and incidence; however, no further explanation was provided.

\subsubsection{Influence of Higher FSTI (4\%) on Loading}

Figure 5.11 shows the loading distribution for all three airfoils at both values of FSTI examined. As discussed in Section 5.3.2, higher FSTI promotes transition, and hence reduces the size of laminar separation bubbles. However, in the absence of the bubble, there is very little effect of FSTI on the overall pressure distributions.

At a Reynolds number of 150,000, FSTI of $4 \%$ results in a very small reduction of the bubble size for PAKB. However, at $R e_{i} 25,000$, there is an appreciable effect of higher FSTI reflected in reduction of the bubble size. Although for both turbulence intensities the flow separates essentially at the same point, the reattachment point moves upstream for FSTI of $4 \%$. A more significant effect can be seen on the critical Reynolds number for PAKD-A, where the stall is postponed from Reynolds number of approximately $75,000-80,000$ to about $35,000-40,000$. Therefore, higher FSTI results in significant delay of stall and improvement of the airfoil's performance.

Mahallati (2003) suggested the presence of a pressure side separation bubble on the PAKB airfoil at lower turbulence levels (0.4\% and 1.5\%). However, the bubble seems to disappear at FSTI of $4 \%$. No such bubble was reported for PAKD-A by Dai (2004) and did not seem to exist at the pressure side of the PAKD-F airfoil at the design incidence. 

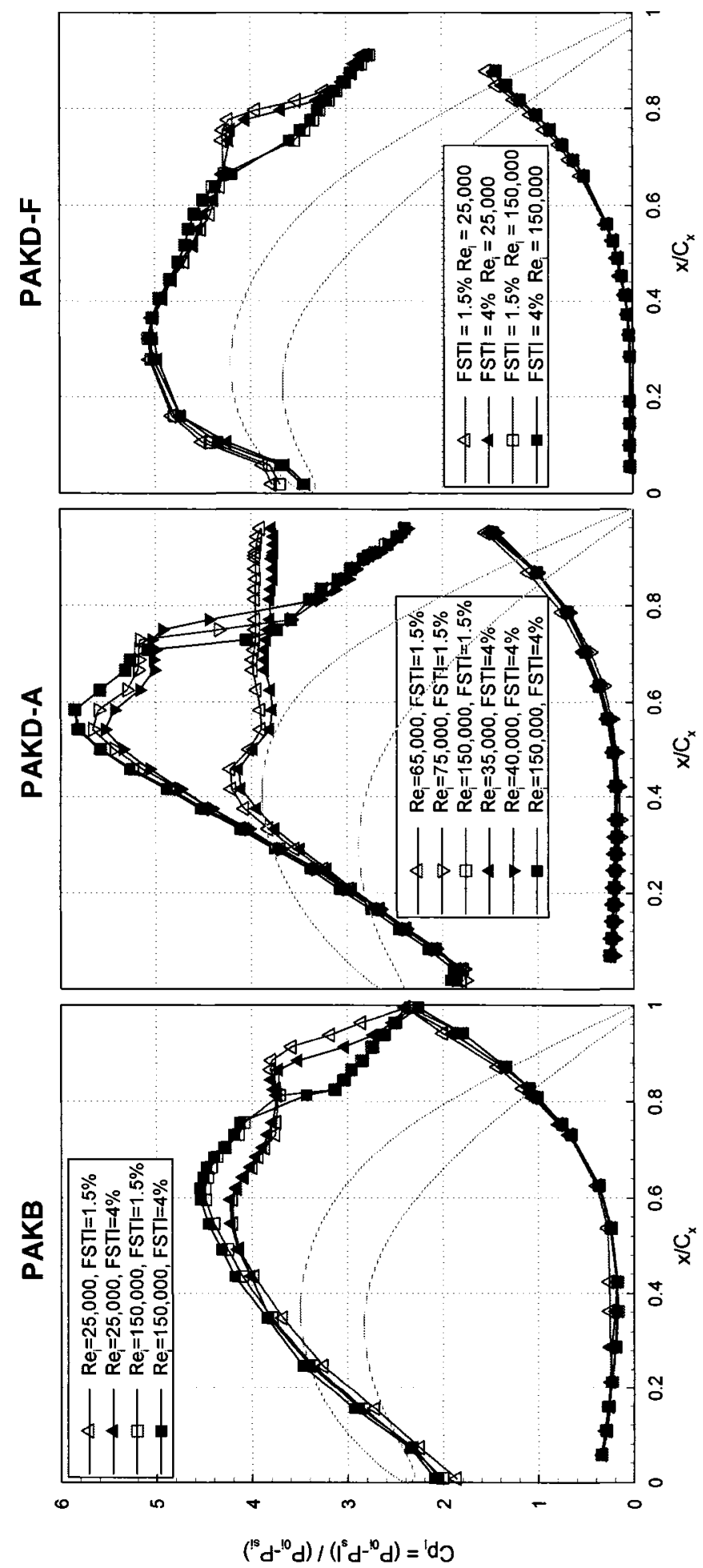

Figure 5.11: Comparison Between Loading Distributions for 1.5\% and 4\% FSTI 


\subsubsection{Mixed-out Profile Losses}

Figure 5.12 shows a comparison of mixed-out profile losses for all three blades. At high Reynolds numbers, the difference between the loss for different airfoils is small, but it can be seen that PAKD-A has higher losses than both PAKB and PAKD-F. The poor performance of PAKD-A is even more evident as the Reynolds number is reduced, since profile losses rise more rapidly than for the other two airfoils. The reason for this comes from the fact that PAKD-A is stalled at lower Reynolds numbers. As discussed in the previous section, the stall is delayed by increasing the FSTI. Hence, for the higher FSTI, the sharp rise in losses occurs at a lower Reynolds number $(35,000-40,000)$.

The higher losses for PAKD-A are consistent with observations of Howell et al. (2001) who showed that increased loading on aft-loaded blade profiles results in large back surface deceleration that leads to higher losses. In Section 2.5, it was shown that the entropy generation in the boundary layer varies with $U_{b l}{ }^{3}$. As could be seen from the loading plots, the PAKD-A airfoil has higher values of $C_{P} l$ around the suction peak compared to the other two airfoils, and thus the higher velocities at the edge of the boundary layer, $U_{b l}$, in that region. The higher loading and velocities are expected to generate higher losses. This may explain the higher loss production for PAKD-A. However, a somewhat surprising finding was that the losses for PAKB and PAKD-F are not very different, although PAKD-F has a higher loading level than PAKB. A possible explanation for the similar losses is given next.

Since PAKB and PAD-F blades do not experience stall in the range of Reynolds numbers examined, the rise in losses with decreasing Reynolds number is gradual. The main difference between PAKB and PAKD-F at $R e_{i}$ of 25,000 comes from the 


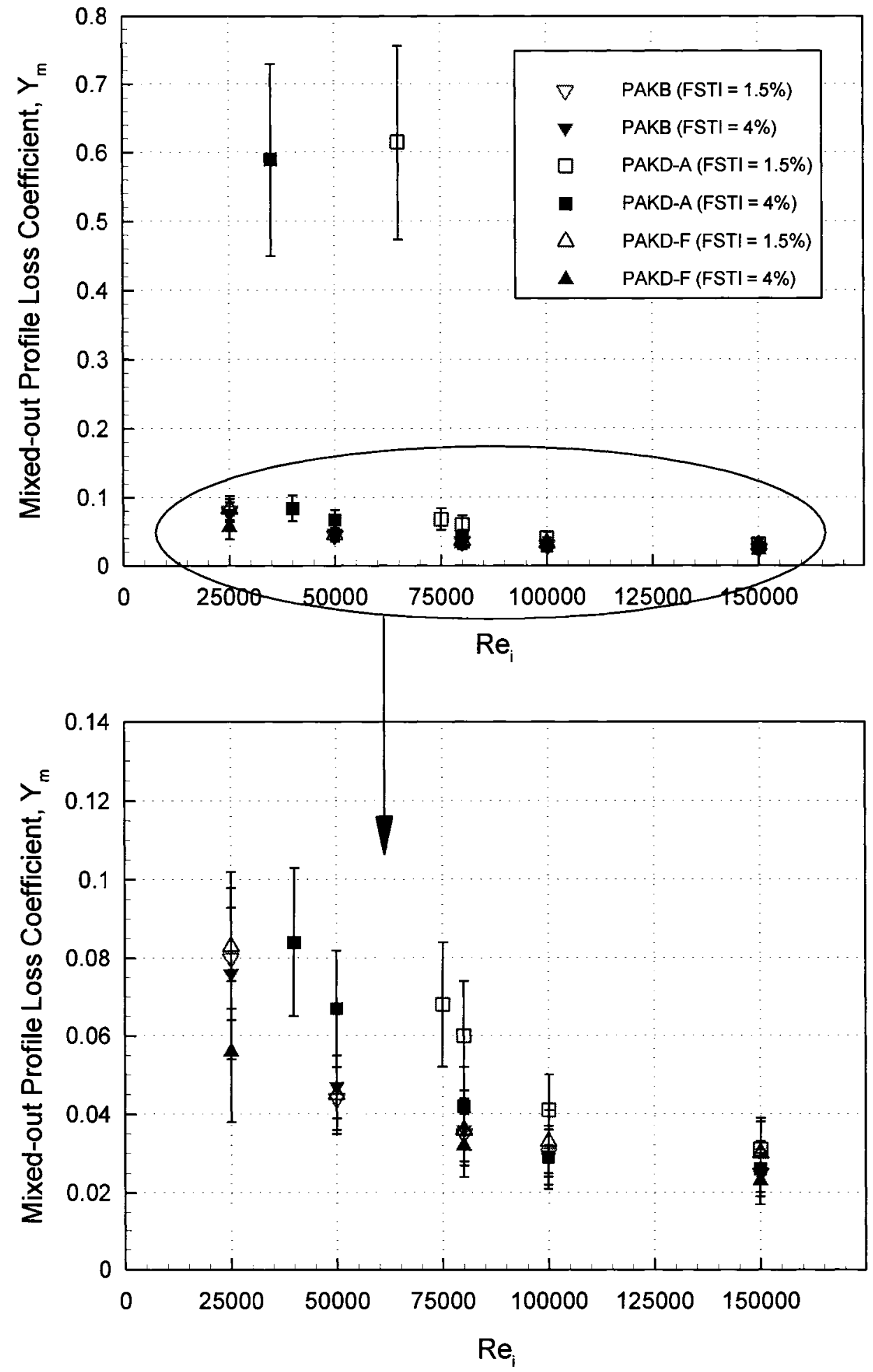

Figure 5.12: Comparison of Profile Losses 
bubble size. It should be noted that at higher Reynolds numbers, the PAKB has separation bubbles present on both the suction and the pressure sides of the airfoil, while PAKD-F has no separation bubbles. It is widely accepted the suction side separation bubbles contribute to the increased loss production. However, Brear et al. (2002) have shown that the pressure side bubbles can also lead to additional loss generation. This might be an explanation for the smaller-than-expected difference in losses between PAKB and PAKD-F.

Figure 5.13 compares the measurements with the empirical correlations of Kacker and Okapuu (1982) and Zhu and Sjolander (2005). It should be noted that the correlations are valid only when the flow is attached at the trailing edge (i.e. no stall). Thus, the correlations are not intended to capture the high losses due to blade stall. The losses for the stalled cases are plotted on the top edge of the figure. The new correlation by Zhu and Sjolander (Z-S) shows a better agreement than Kacker and Okapuu with the current data. As mentioned, the Z-S correlation was developed in part with the current measurements, so better agreement was expected.

\subsubsection{Mixed-out Flow Angles}

Figure 5.14 shows the outlet flow angles for all airfoils. At highest the Reynolds number examined $(150,000)$, the difference in outlet flow angles between three airfoils is almost within the experimental uncertainty. Both PAKB and PAKD-A show a trend of outlet flow angle decrease with Reynolds number reduction. This decrease is more gradual for PAKB than PAKD-A. A sharp decrease in the outlet flow angle can be seen for PAKD-A once the blade stalls. However, PAKD-F shows the opposite trend (the flow angle increases with reduction in Reynolds number). As mentioned previously, it is believed that low AVR values may be partly responsible for this 

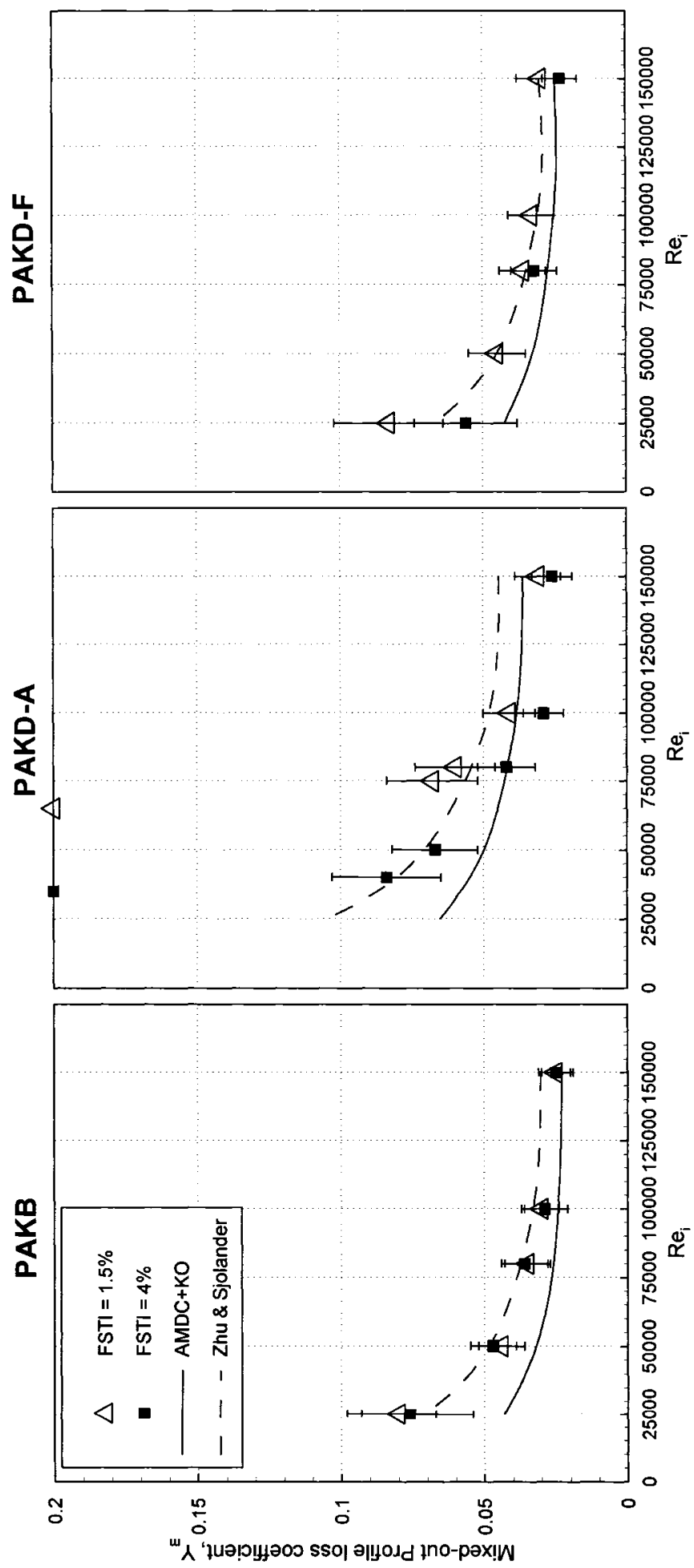

Figure 5.13: Comparison of Profile Losses with Correlations 


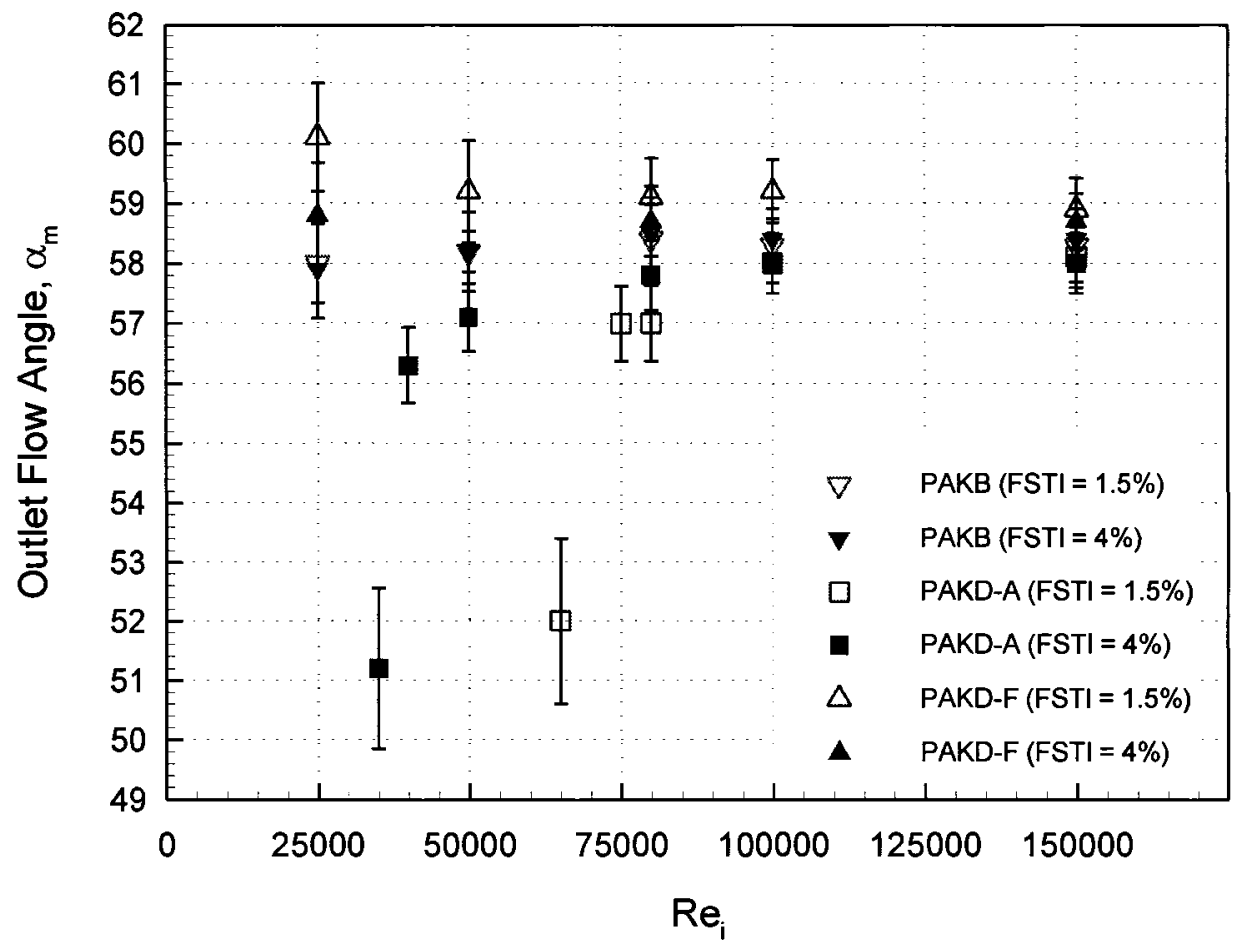

Figure 5.14: Outlet Flow Angle Comparison

unexpected trend.

\subsection{Summary of Design Incidence Performance}

This chapter presented the results of both current and previous experimental investigations on the PAKB and PAKD airfoils. The airfoils were tested under steady conditions at design incidence. Two different FSTI levels, $1.5 \%$ and $4 \%$, were

examined. Reynolds numbers based on inlet velocity and axial chord from 25,000 to 150,000 were investigated.

The tests performed at both FSTI of $1.5 \%$ and $4 \%$ showed that PAKB and PAKD- 
F generated comparable losses, while PAKD-A produced higher losses compared with the previous two airfoils.

The presence of the separation bubble on the PAKB and PAKD-A airfoils was detected at all conditions examined. No separation bubble seemed to be present on the suction side of the PAKD-F airfoil for Reynolds numbers of 50,000 or higher. However, it was recommended that this finding should be confirmed with either flow visualization or hotfilm measurements. The separation bubbles for all airfoils increased in size with reduction in either Reynolds number or FSTI.

PAKD-A stalled between Reynolds numbers of 75,000 and 80,000 at FSTI $1.5 \%$. The stall was delayed until Reynolds number of about 35,000 to 40,000 at higher FSTI (4\%). The stall resulted in a significant loss increase and under-turning of the flow. PAKB and PAKD-F did not stall at any of the conditions presented in this chapter. The stall of the PAKD-A airfoil was found to be unsteady for a small range of Reynolds numbers $(75,000-80,000$ for FSTI of $1.5 \%)$. The periodic stall was attributed to the oscillations between the short and long bubble type (also known as "bursting"). However, it is not clear why the long bubble fails to reattach. A possible explanation may be attributed to the strong adverse pressure gradient on the aft portion of the airfoil. 


\section{Chapter 6}

\section{Performance for Steady Flow at Off-Design Incidence}

\subsection{Introduction}

The previous chapter discussed the Reynolds number sensitivity at the design incidence. This chapter will present the effect of incidence variation at a fixed Reynolds number (based on the axial chord and inlet velocity) of 80,000 for $1.5 \%$ and $4 \%$ FSTI levels. Table 6.1 summarizes the test matrix for this part of the study. It should be noted that at the higher turbulence intensity, the measurements at $-5^{\circ}$ were not performed. Due to the close proximity of Grid II which was used to generate the turbulence intensity of $4 \%$, the test section (in particular the floating wall shown in Figure 3.2, Section 3.2.2) would require modifications. Since the airfoil performance is more sensitive to positive incidence, it was decided to perform the measurements at the design and positive incidence only. 
Table 6.1: Summary of Test Cases Presented

\begin{tabular}{|c|c|c|c|c|c|c|}
\hline & \multicolumn{2}{|c|}{ PAKB } & \multicolumn{2}{c|}{ PAKD-A } & \multicolumn{2}{c|}{ PAKD-F } \\
\hline $\mathrm{i}[\mathrm{deg}] /$ FSTI & $1.5 \%$ & $4 \%$ & $1.5 \%$ & $4 \%$ & $1.5 \%$ & $4 \%$ \\
\hline-5 & $\mathrm{~L} / \mathrm{P}$ & & $\mathrm{L} / \mathrm{P}$ & & $\mathrm{L} / \mathrm{P}$ & \\
\hline 0 & $\mathrm{~L} / \mathrm{P}$ & $\mathrm{L} / \mathrm{P}$ & $\mathrm{L} / \mathrm{P}$ & $\mathrm{L} / \mathrm{P}$ & $\mathrm{L} / \mathrm{P}$ & $\mathrm{L} / \mathrm{P}$ \\
\hline+5 & $\mathrm{~L} / \mathrm{P}$ & $\mathrm{L} / \mathrm{P}$ & $\mathrm{L} / \mathrm{P}$ & $\mathrm{L} / \mathrm{P}$ & $\mathrm{L} / \mathrm{P}$ & $\mathrm{L} / \mathrm{P}$ \\
\hline
\end{tabular}

L: Loading P: Profile Loss

As described in Section 4.2.1, the Reynolds number based on the inlet velocity was used to set the operating point for practical reasons. At off-design incidence, the velocity triangles are changed so that the blade turns the flow more or less depending on whether the incidence is positive or negative. As a consequence, the velocity ratio changes as well. As can be seen from Figure 6.1, the Reynolds number based on exit velocity, $R e_{e}$, increases inversely with incidence for a fixed value of $R e_{i}$. Thus, the negative incidence cases have been tested under slightly higher $R e_{e}$ and vice versa for positive incidence. Although the effect of this variation was not substantial $( \pm 6 \%)$, it is recommended that $R e_{e}$ should be kept constant for off-design incidence measurements in the future, especially when a wide range of incidences is examined. For a large off-design incidence, a variation in $R e_{e}$ would be much larger; for example, at an incidence of $-20^{\circ}$, the $R e_{e}$ would be $30 \%$ higher than at the design incidence.

The chapter begins with a discussion of loading distributions under two values of off-design incidences, $-5^{\circ}$ and $+5^{\circ}$. The axial velocity ratios will be presented in Section 6.3, followed by the discussion of the profile losses in Section 6.4. In addition, the off-design losses will be compared to the loss correlations of Moustapha et al. (1990) and Benner et al. (1997). The variation of outlet flow angles with incidence will be shown in Section 6.5. Finally, the results and conclusions will be summarized in the last section. 


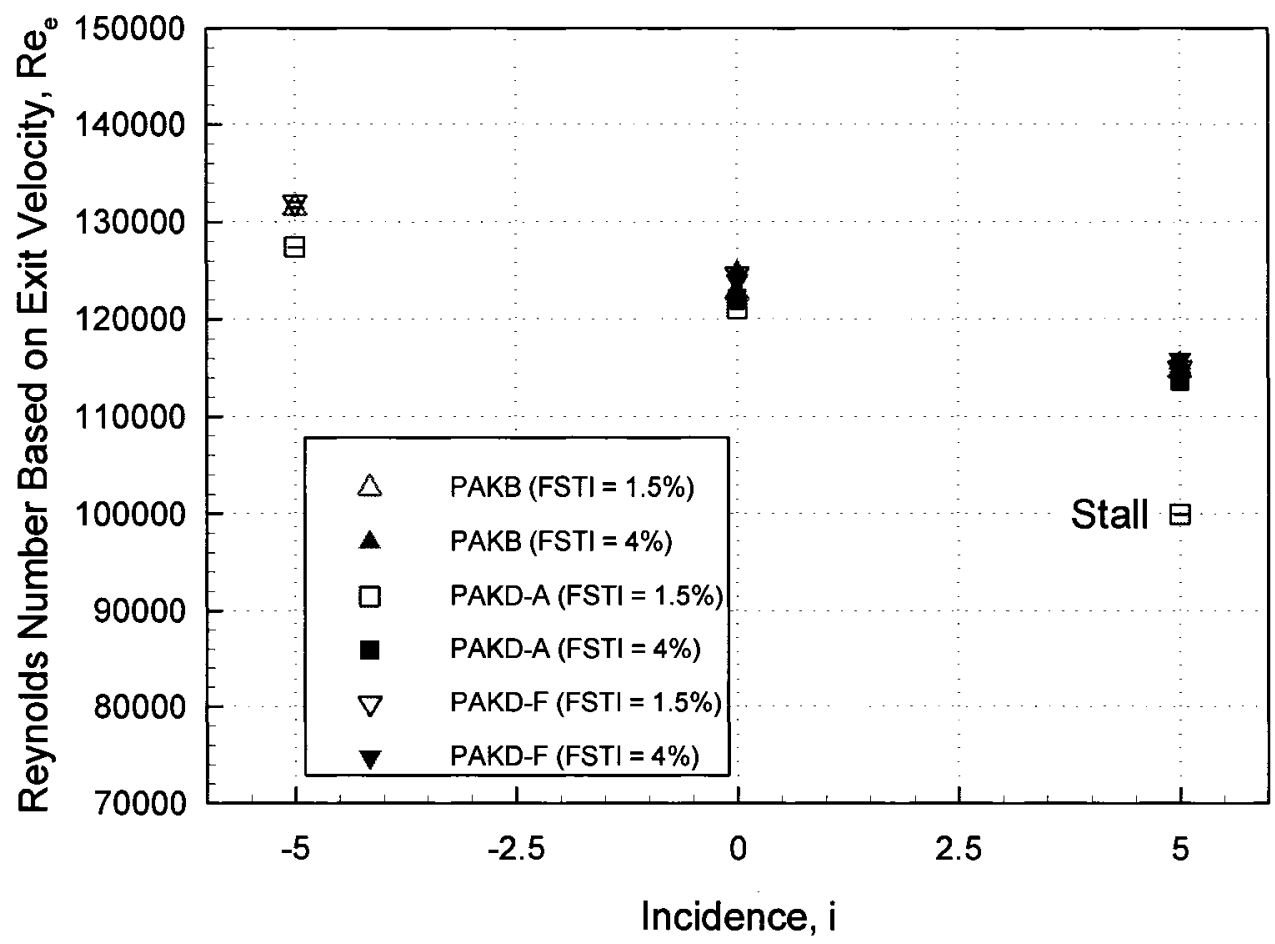

Figure 6.1: Variation of $R e_{e}$ with Incidence

(All measurements shown were performed at $R e_{i}=80,000$ )

\subsection{Loading Distributions}

\subsubsection{Influence of Varying Incidence on Loading at FSTI of $1.5 \%$}

All pressure coefficients used for loading measurements were normalized by the outlet dynamic pressure (Equation 4.19), since this definition provides a better visual impression of the Zweifel coefficient variation. Appendix B provides a brief discussion on this topic. 
Blade surface pressures for all three incidences and airfoils are shown in Figure 6.2. The discussion will begin with the PAKB airfoil, since it is a baseline airfoil for this family and then it will extend to the other two airfoils.

As the incidence is increased, a noticeable overspeed develops on the suction side near the leading edge of the PAKB airfoil. The stagnation point moves toward the pressure side for positive incidence and a larger proportion of the leading edge becomes part of the suction surface. The resulting high streamline curvature of the leading edge results in a rapid acceleration (overspeed), followed by a short length of strong adverse pressure gradient and possibly a separation bubble. This velocity overshoot can be responsible for high local loss production. For the negative incidence, it appears that the overspeed is absent.

The pressure distribution on the rear part of the suction surface and the location of the suction peak itself do not change appreciably with the incidence. Approximately a $2 \%$ increase in the magnitude of suction peak was observed with positive incidence due to the higher loading on the front portion of the suction surface. However, very similar pressure gradients are encountered on the aft portion of the blade. Thus both the size and the location of the separation bubble are practically the same for all three incidences.

As mentioned in Section 5.4.1, at design incidence, low FSTI (1.5\%) and $R e_{i}$ of 80,000 , PAKD-A is already on the verge of stall. Similar to PAKB, there is little change between design and $-5^{\circ}$ incidence in the pressure distributions on the pressure side of the airfoil and on the rear part of the suction surface. However, at an incidence of $+5^{\circ}$, the flow undergoes a massive separation at about $50 \%$ of axial chord. The area enclosed by the loading curves for the stalled case is greater than for the other two cases. Since this area corresponds to the $\mathrm{Z}_{\text {weifel }}$ coefficient, $Z_{w}$, (a discussion on 

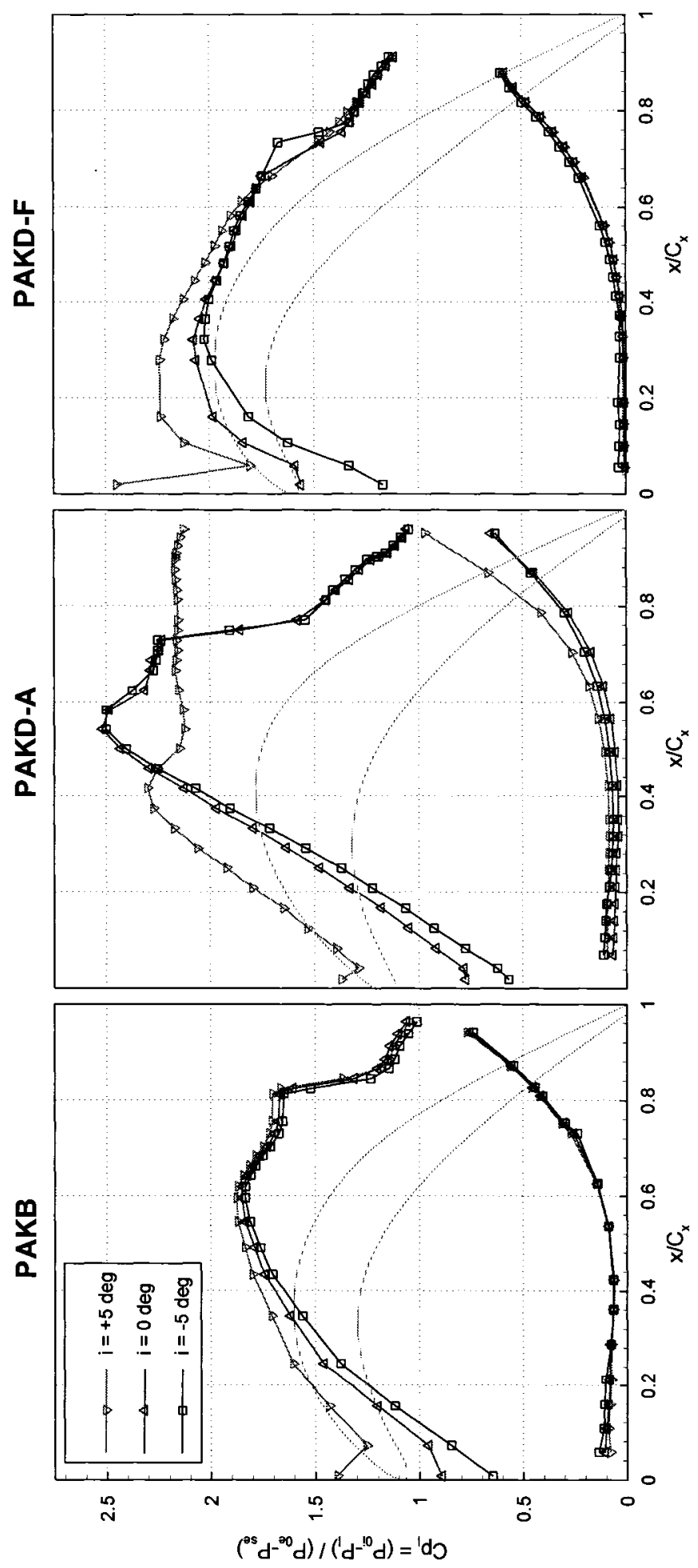

Figure 6.2: Comparison of Loadings at FSTI $=\mathbf{1 . 5 \%}$ 
this topic is given in Appendix B), the Zweifel coefficient is also significantly higher as can be seen in Figure 6.3.

Similar to PAKB, PAKD-F does not stall at the off-design incidences that were examined. For the positive incidence of $+5^{\circ}$, the suction peak is shifted forward from about 0.32 to about $0.24 C_{x}$, and its magnitude is increased by approximately $7 \%$. Due to the position of the mounting rod and thinness of the blade, it was not possible to instrument the blade with static pressure taps in this region, resulting in somewhat increased uncertainty in both position and magnitude of the suction peak. At negative incidence of $-5^{\circ}$, a separation bubble is visible on the suction side of the

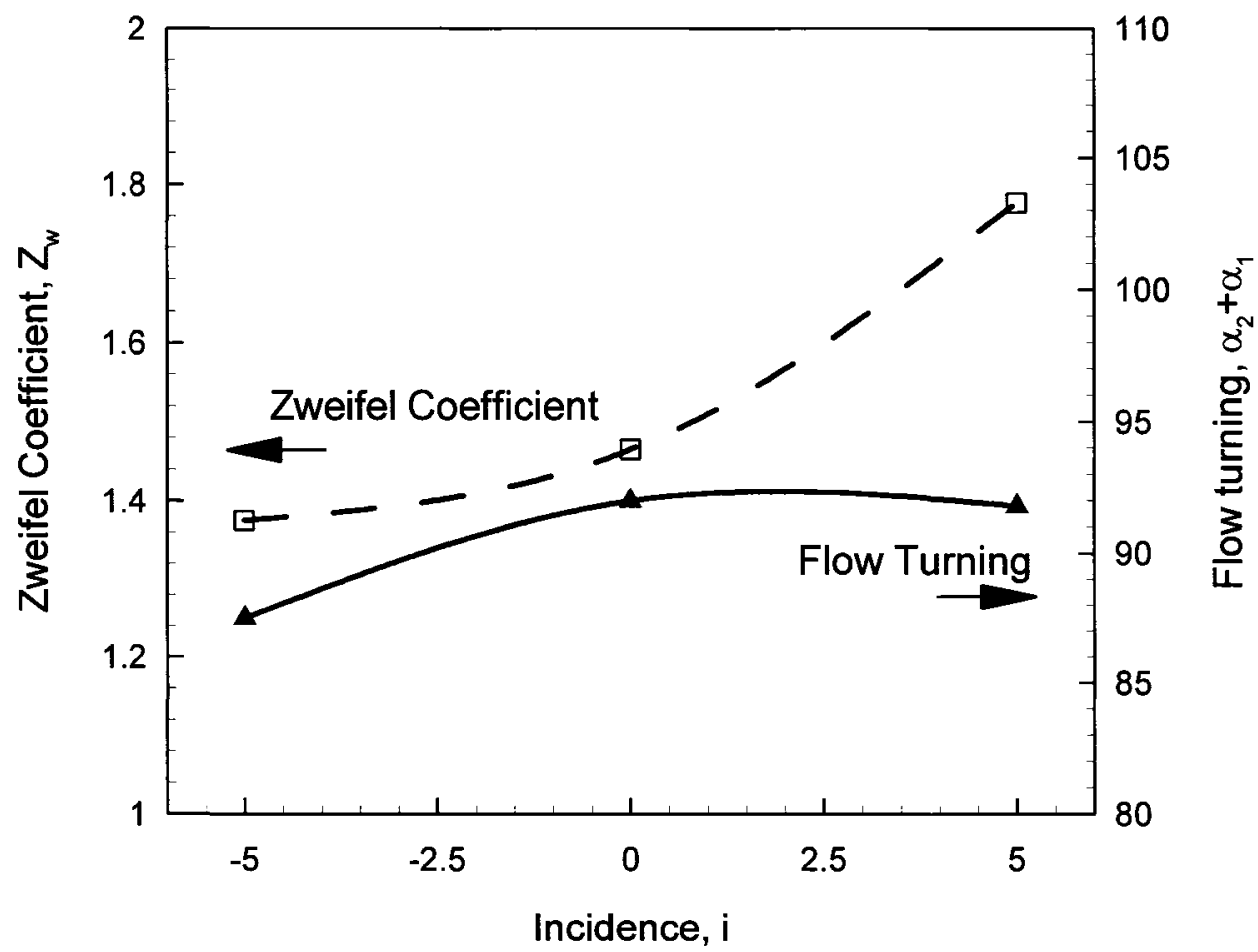

Figure 6.3: PAKD-A: Zweifel Coefficient and Flow Turning Variation $(\mathrm{FSTI}=1.5 \%)$ 
PAKD-F airfoil as can be seen in Figure 6.2.

Since the appearance of a separation bubble at negative incidence was somewhat unexpected, additional tests were performed. A range of different Reynolds numbers varying from 35,000 to 90,000 was examined. In Section 5.3.1, it was shown that at the design incidence, a separation bubble does not appear until the Reynolds number is reduced to 45,000. From Figure 6.4, it can be seen that for the negative incidence the bubble appears for $R e_{i}$ below 90,000 . The bubble grows steadily as the Reynolds number is decreased, as was the case at the design incidence. Even at $R e_{i}$ of 35,000, the bubble continues to reattach well before the trailing edge, making PAKD-F airfoil very resistant to stall.

As discussed in Chapter 5, it is believed that the transition from laminar to turbulent flow on the suction side of the PAKD-F airfoil occurs before the flow could separate for Reynolds numbers of 80,000 and above at design incidence. If the bubble appears at an incidence of $-5^{\circ}$, that suggests that the transition point has moved downstream at negative incidence. Thus the flow remains laminar until it encounters the stronger adverse pressure gradient at about $0.67 C_{x}$ and then separates. Obviously the pressure gradient "history" is the reason for the changes in the transition process. Although the adverse pressure gradients on the suction surface for both the design and $-5^{\circ}$ incidence are very similar, the length of the mild adverse pressure gradient for the design incidence and $+5^{\circ}$ is longer, as was shown in Figure 6.2. This may have resulted in earlier transition for the latter two cases. Thus, when the flow encountered the stronger adverse pressure gradient beginning at about $0.67 C_{x}$, it may already have been turbulent, and therefore did not separate. On the other hand, at the negative incidence the transition may have been delayed, so that the boundary layer was still laminar when it encountered the stronger adverse pressure gradient, resulting 


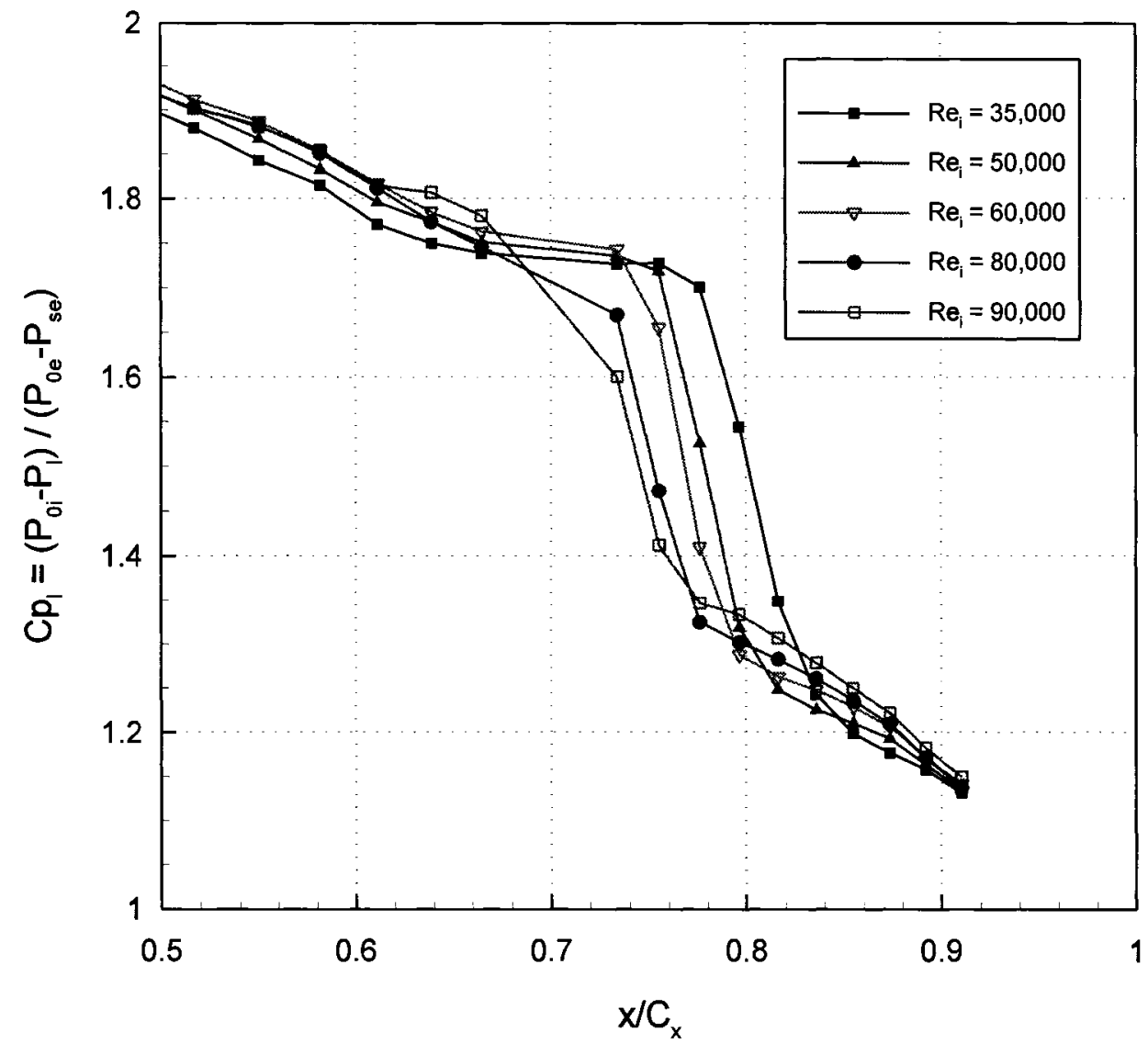

Figure 6.4: Bubble Growth on PAKD-F at $\mathrm{i}=\mathbf{- 5}^{\circ}$ and FSTI $=\mathbf{1 . 5 \%}$

in the laminar separation and formation of the bubble.

\subsubsection{Influence of Higher FSTI (4\%) on Loading}

As mentioned in Chapter 5, the main effect of elevated FSTI on the loading distribution is reflected in the reduction in size of the separation bubble, if one is present. If there is no bubble, the loading at elevated FSTI levels shows no appreciable influence of FSTI. 

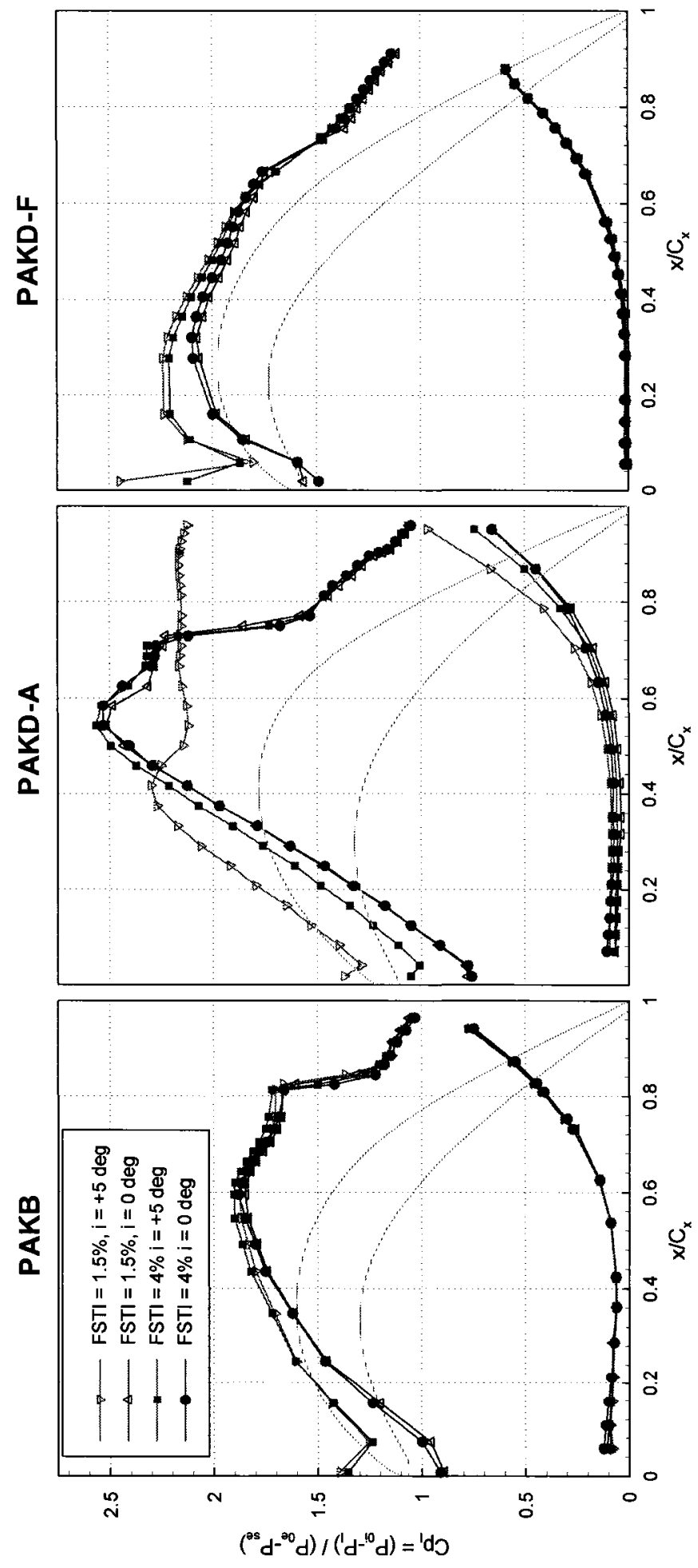

Figure 6.5: Comparison of Loadings for Two Incidences and FSTIs 
The influence of the higher FSTI (4\%) on loadings at design and $+5^{\circ}$ incidence is shown in Figure 6.5. As noted in Section 6.1, measurements were not made for $-5^{\circ}$ of incidence for the higher FSTI. It can be seen that the flow is attached for both incidences for all three blades.

The size of the separation bubble on the PAKB airfoil seems to be slightly reduced with the increase in the turbulence intensity, but the effect is small. A dramatic effect of the increase in FSTI is seen at $+5^{\circ}$ incidence for the PAKD-A airfoil. While at FSTI of $1.5 \%$ the blade is stalled, at $4 \%$, the flow is reattached. As was shown in Chapter 5, the higher FSTI promotes transition and delays stall. Therefore, it is consistent with observations made at design incidence that the higher FSTI has helped the flow to reattach at an incidence of $+5^{\circ}$. The loadings are very similar for the PAKD-F airfoil for the two free stream turbulence intensities except near the leading edge. At the the positive incidence, the overspeed near the leading edge is $15 \%$ lower at the higher compared with the lower FSTI. However, the pressure taps may not be capturing completely the very localized overspeed at the leading edge.

\subsection{Axial Velocity Ratio}

The axial velocity ratios (AVR) for all measurements presented in this chapter are plotted in Figure 6.6. The values range between 0.976 and 1.009, with the lower values occurring for negative incidence. Rodger (1992) found that flow divergence (AVR less than 1.0) increases the losses but that the effect is smaller at negative incidence compared with design and positive incidence. 


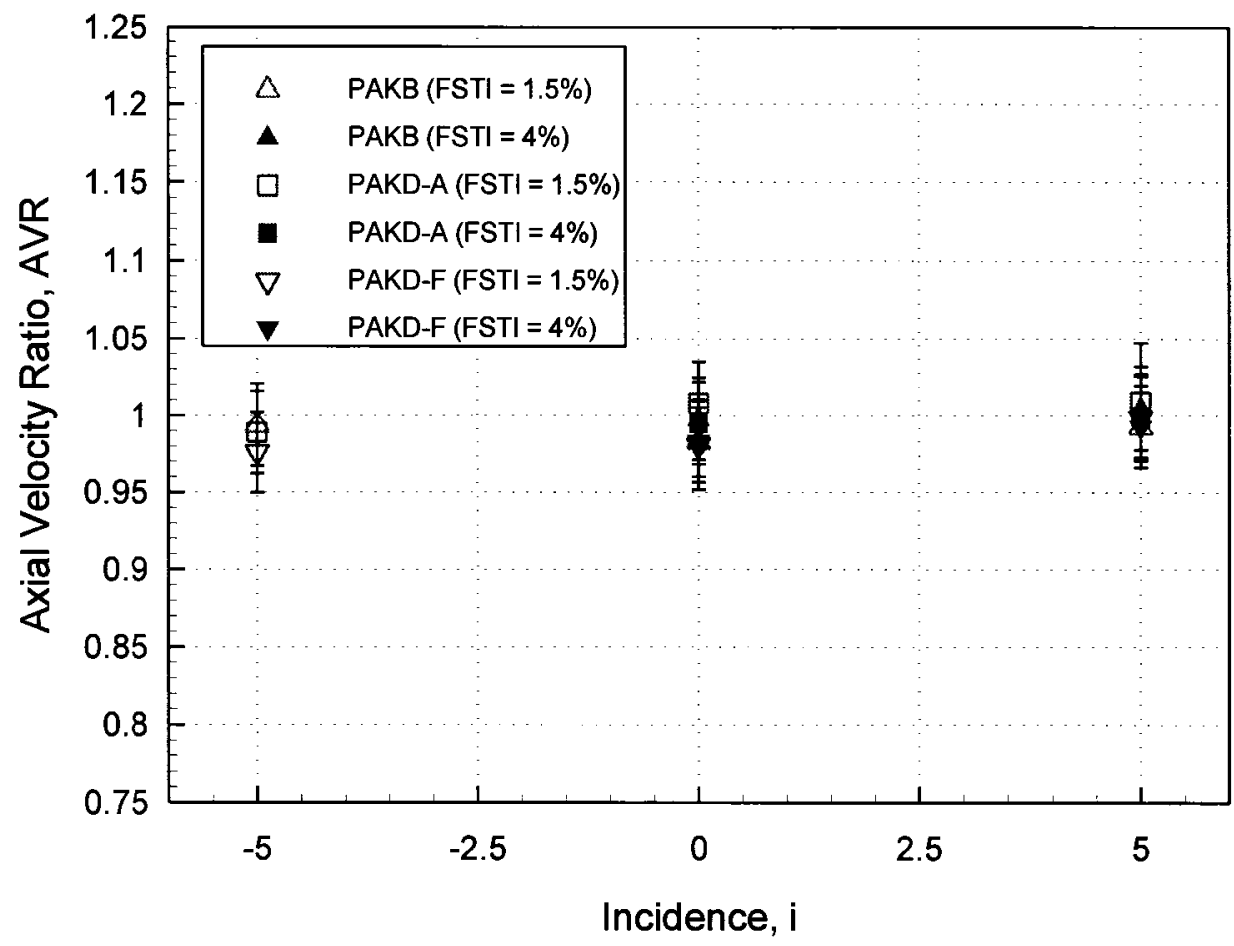

Figure 6.6: Axial Velocity Ratios at Midspan

\subsection{Mixed-out Profile Losses}

\subsubsection{Influence of Varying Incidence on Profile Losses}

The behavior of losses at off-design incidence can normally be described through the classical turbine loss bucket, with a large increase in losses at positive incidence and relatively smaller increase in losses at negative incidence. Although the current study examined a small variation of incidence $\left( \pm 5^{\circ}\right)$, a similar trend can be observed from the mixed-out total pressure loss coefficients plotted in Figure 6.7. It should be noted that the trendlines are shown to "guide" the eye only.

As mentioned in Section 2.5, loadings and profile losses are closely related. Denton 

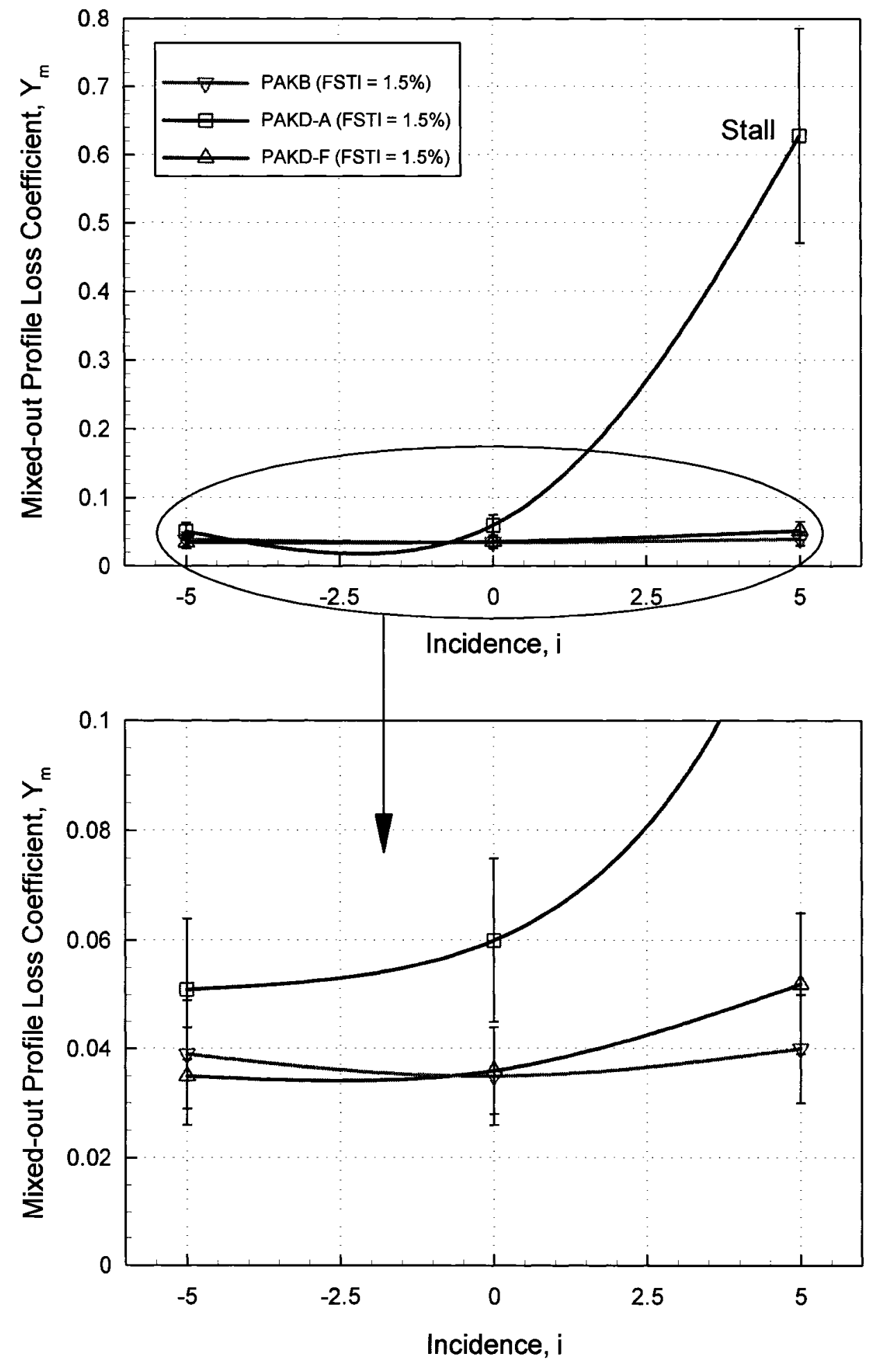

Figure 6.7: Mixed-out Losses for FSTI $=\mathbf{1 . 5 \%}$ 
(1993) has shown that the loss production in the attached boundary layer varies roughly with the cube of the velocity at the edge of the boundary layer. Figure 6.8 shows velocity distributions on the suction surface for PAKD-F at FSTI of $1.5 \%$ and the three values of incidence. The conclusions that will be drawn here are applicable for the other two airfoils as well.

As indicated, the two main contributors for the total pressure loss rise at the positive incidence are believed to be the overspeed and the consequent separation

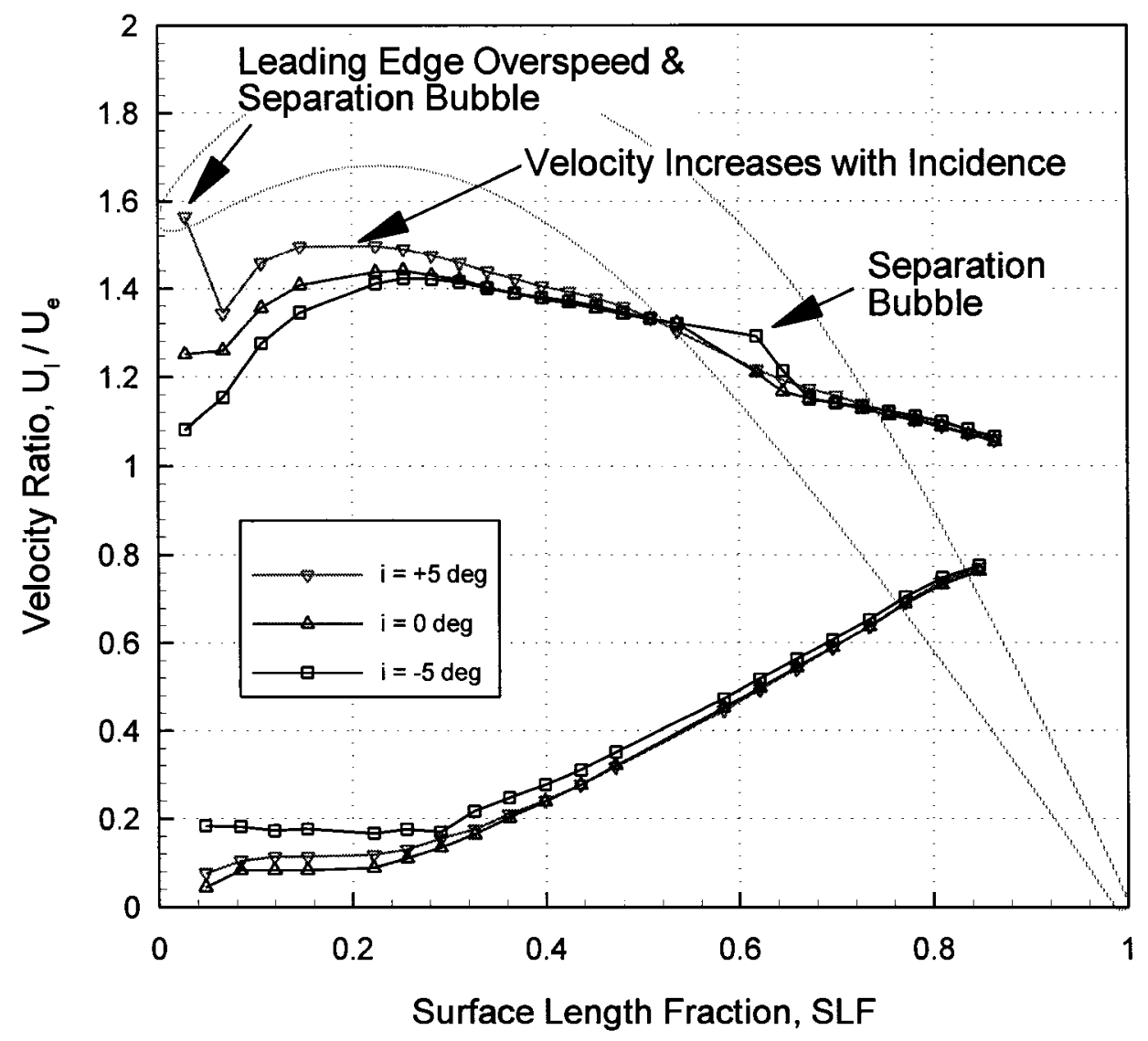

Figure 6.8: Main Contributors to Loss Production on the Suction Surface (Velocity Ratios here are shown for PAKD-F at FSTI $=1.5 \%$ ) 
bubble near the leading edge and the increase in velocity on the front portion of the suction surface. Since the velocity levels are low on the pressure side of the airfoil and they don't change much with incidence, the increased loss production is influenced mainly by the loss generation in the boundary layer on the suction side. A noticeable separation bubble that develops on the aft portion of the suction side of PAKD-F at negative incidence increases the loss production for that case. It is not clear whether a separation bubble is present on the pressure side of the PAKD-F airfoil at $-5^{\circ}$ (the region of nearly constant velocity that extends up to $0.3 C_{x}$ ).

It should be noted that a massive separation and formation of an open bubble discussed earlier is responsible for a sharp increase in losses for PAKD-A at positive incidence for this FSTI.

The decrease in losses for PAKD-A and PAKD-F with negative incidence is thought to be mainly due to the lower velocities on the suction surface and the leading edge. Should the incidence be decreased further, the losses would be expected to rise as shown by Tremblay (1990) and Goobie (1989). In their measurements, the losses slightly decreased at $-5^{\circ}$ compared with design incidence, and then gradually rose as the incidence was decreased further. On the other hand, when compared to the design incidence performance, the profile loss increased for PAKB already at $-5^{\circ}$. As previously mentioned, Mahallati (2003) reported a pressure side separation bubble on the PAKB airfoil at design incidence and lower FSTI. This separation bubble is expected to generate higher losses at negative incidence. Measurements by Brear et al. (2002) also show an increase in losses generated by the pressure side separation bubble at negative incidence.

As was discussed in Section 6.1, the negative incidence cases were tested at Reynolds number based on the exit velocity, $R e_{e}$, of about 130,000 compared with 
122,000 at the design incidence. The higher $R e_{e}$ encountered at the negative incidence may have slightly reduced the losses. The PAKD-A airfoil might be affected the most due to the fact that at $R e_{i}=80,000\left(R e_{e}\right.$ of 121080$)$ it is very close to its critical Reynolds number, which was estimated lie between 75,000 and 80,000 . Thus, it is more sensitive to the Reynolds number variation than the other two airfoils.

\subsubsection{Influence of Higher FSTI (4\%) on Profile Losses}

As turbulence intensity is increased, the losses do not change significantly for PAKB and PAKD-F (Figure 6.9), and the differences between the two levels of FSTI are within the limits of the estimated experimental uncertainties.

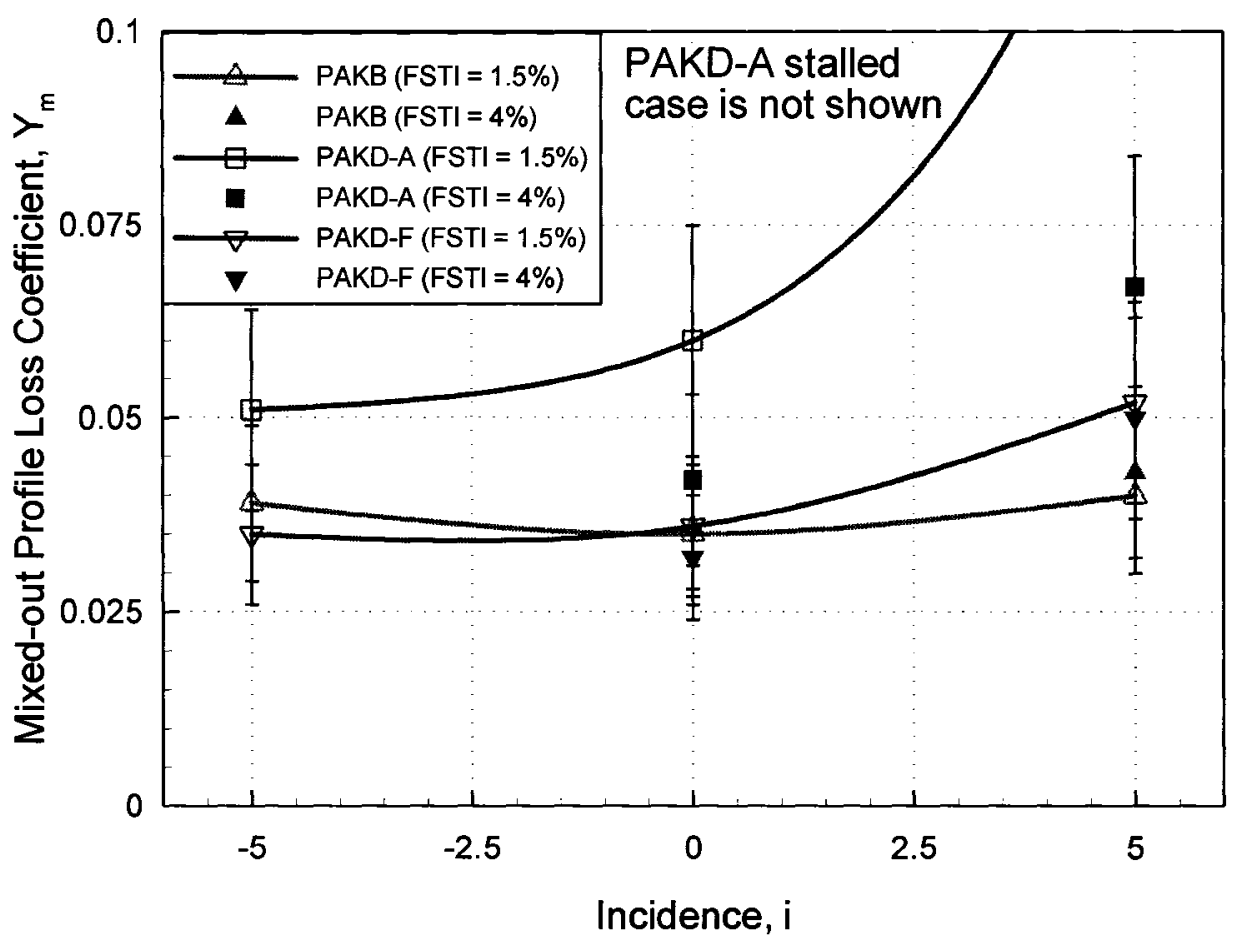

Figure 6.9: Comparison of Mixed-out Losses for both FSTI Levels 
A significant decrease in losses due to the higher FSTI is evident for PAKD-A only. At FSTI of $4 \%$, the flow reattaches at the positive incidence, reducing the losses by a factor of 9 .

\subsubsection{Comparison with Off-Design Correlations for Profile Losses}

The off-design profile loss correlations of Benner et al. (1997) and Moustapha et al. (1990), described in Section 2.8, were compared with the current experimental data. However, it should be noted that both the Moustapha et al. and Benner et al. correlations use the leading edge circle diameter as one of the main correlating parameters. In addition, Benner et al. also correlate the losses on the inlet wedge angle. Modern turbine airfoil profiles often do not use leading edge circles and therefore the correlations are strictly speaking not applicable to them.

The details of the profile generation for the PAKB and PAKD airfoils are not fully known, but they are believed to include neither leading edge circles nor inlet wedge angles. Thus, in order to evaluate the fore mentioned correlations, the leading edge diameter and the corresponding wedge angle were estimated based on the available airfoil coordinates. In addition, the obtained values for $d / s$ (leading edge diameter normalized by blade pitch) are lower than the lowest values used in developing both correlations. For example, $d / s$ for the PAKDs was estimated to be 0.026 , while the minimum $d / s$ in Benner's database was 0.039 . The remainder of this section will discuss the values of off-design profile losses obtained from these two correlations.

Figure 6.10 compares the measurements with profile loss correlations of Moustapha et al. (1990) and Benner et al. (1997). Values that either exceeded $\left|\Delta \phi^{2}\right|$ of 0.04 or 
were less than 0.0 have been plotted along the top or bottom edge of the figure, respectively. The difference between the PAKB and PAKD airfoils shown in the Moustapha et al. (1990) correlation is due to the smaller $d / s$ parameter for the PAKD airfoils. Although the leading edge of all three blades is very similar, the PAKD airfoils have a smaller $d / s$ than PAKB due to the larger pitch.

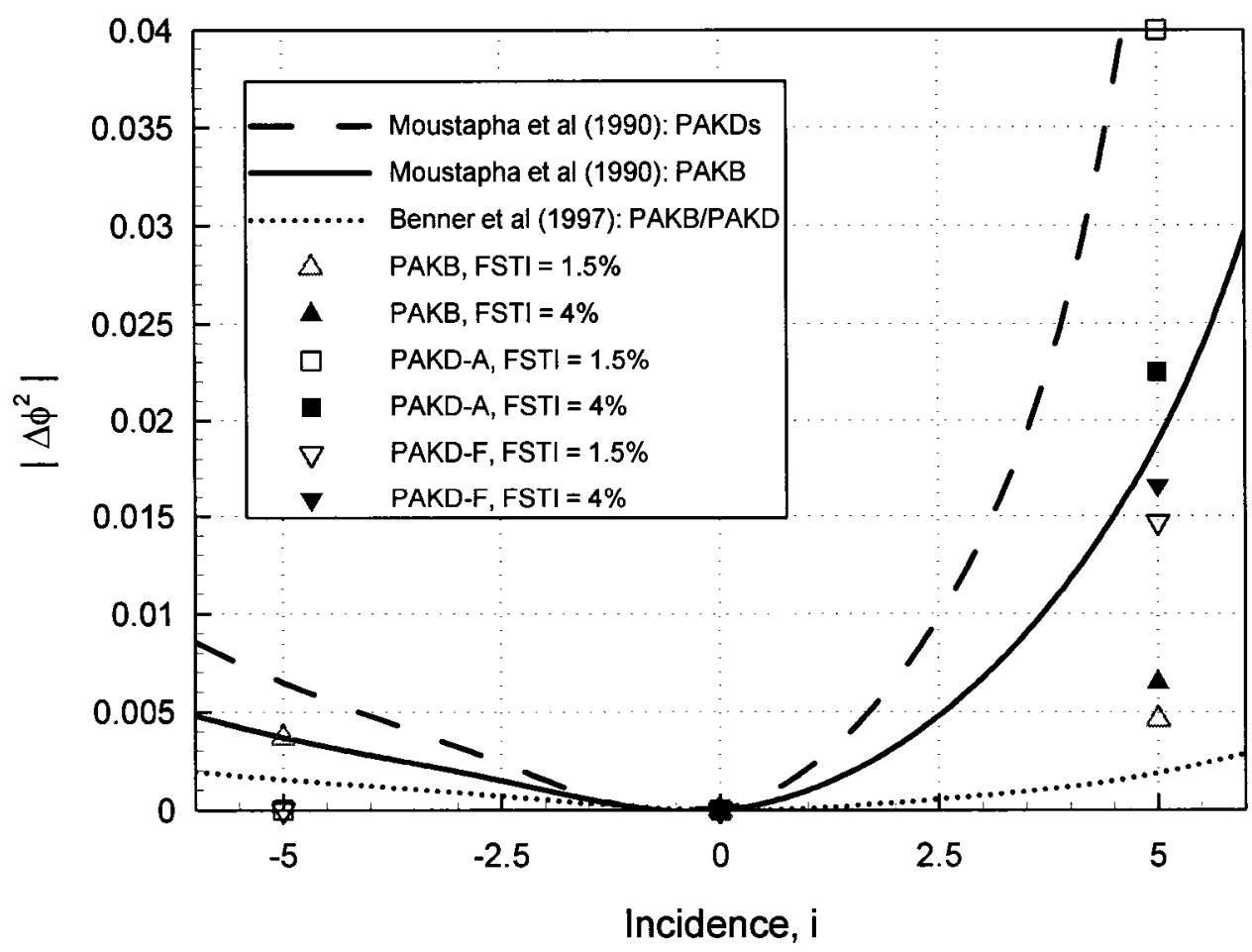

Figure 6.10: Evaluation of Off-Design Correlations

The Benner et al. correlation does not differentiate between PAKB and the PAKDs. The losses follow the same curve, and the reason for this is in the reduced sensitivity to $d / s$ compared to the Moustapha et al. correlation, as discussed in Section 2.8. The agreement between correlations and the experimental data especially for the positive incidence may seem poor. However, one should keep in mind that these correlations are intended for a wide range of incidences and have an estimated 
accuracy of $\partial\left|\Delta \phi^{2}\right|= \pm 0.01$. Nevertheless, the correlations clearly do not capture the off-design loss behavior of the present airfoils. Thus, there is a need for improved correlations that are more applicable to modern turbine airfoils.

\subsection{Mixed-out Flow Angles}

Figure 6.11 shows the outlet flow angles for all the test cases. For all non-stalled cases, the flow angle does not change substantially with the incidence. An average increase in flow angle of approximately $0.25^{\circ}$ with the negative incidence compared with design incidence was observed. Considering an average uncertainty in flow angle measurements of $\pm 0.5^{\circ}$, the outlet flow angle can be considered almost insensitive to

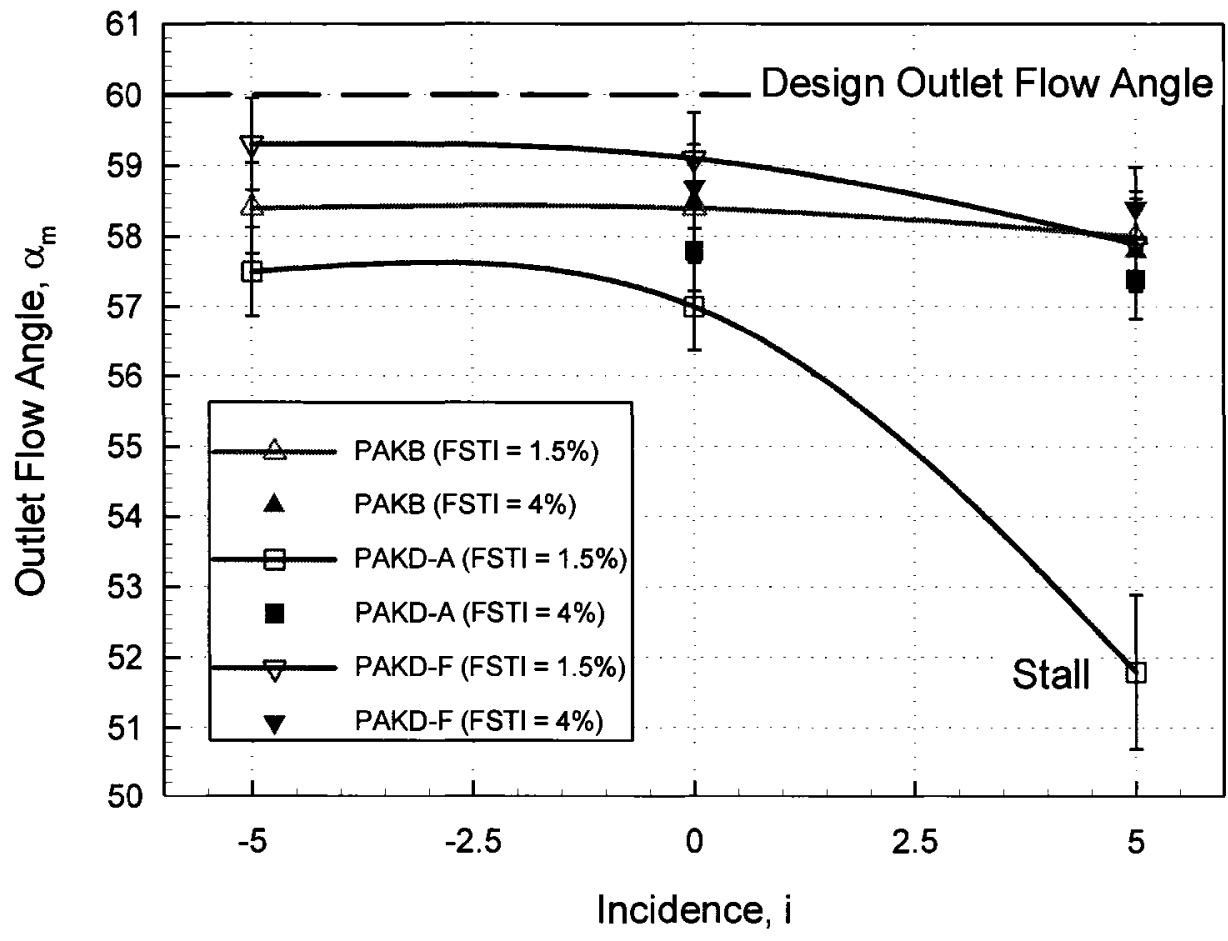

Figure 6.11: Variation of the Outlet Flow Angle with Incidence 
small negative incidence.

On the other hand, a consistent small decrease in the outlet flow angle of about $0.6^{\circ}$ was observed when the incidence was increased to $+5^{\circ}$, provided that flow remains attached at the trailing edge.

\subsection{Summary of Off-Design Performance Study}

Measurements have been made to examine the off-design behavior of all three airfoils at constant Reynolds number based on the inlet velocity and axial chord. In addition to the variation of incidence of $\pm 5^{\circ}$, two FSTI levels (1.5\% and $4 \%$ ) were examined.

The aerodynamic performance reflected through the profile losses and outlet flow angles are less sensitive to negative incidence than positive incidence. The PAKB and PAKD-F airfoils were well behaved at all conditions examined. However, PAKDA stalled at positive incidence under the lower FSTI level. When the turbulence intensity was increased, the flow reattached. The bubble size for PAKB and PAKD-A (apart from the stalled case) did not noticeably change with the incidence. However, a separation bubble did appear on the suction surface of the PAKD-F airfoil at negative incidence. This finding has highlighted the need for better understanding of the transition process on the suction surface side of the PAKD-F airfoil. In all cases, positive (negative) incidence has increased (decreased) the overspeed at the leading edge of the airfoils.

Two loss correlations were examined, the first by Moustapha et al. (1990) and the second one by Benner et al. (1997). Poor agreement between experimental data and 
the correlations was observed. The main difficulty in applying these correlations was due to the fact that the current blades do not use leading edge circles, as assumed in the correlations. Therefore, there is a need to develop alternative correlations that are more applicable to modern profile shapes.

So far all presented measurements have been made under steady conditions only. The next chapter will discuss measurements under under periodically-unsteady conditions. 


\section{Chapter 7}

\section{Performance for}

\section{Periodically-Unsteady Flow at Design Incidence}

\subsection{Introduction}

Blade row interactions and periodically-unsteady wake-passing flows in general have been the subject of a number of studies in the past two decades. An extensive investigation of the unsteady effects on the PAKB airfoil was conducted in the LowSpeed Wind Tunnel facility at Carleton University by Mahallati (2003). Mahallati studied 48 combinations of flow cocfficient, rcduccd frequency, Reynolds number and free stream turbulence intensity. The same experimental set-up was used in the present study to investigate PAKD-A and PAKD-F under periodically-unsteady flow conditions. As in the earlier work and as described in Section 3.2.5, the upstream 
wakes were generated using cylindrical bars moving on a spoked-wheel type wakegenerator.

This chapter will concentrate on the profile losses of PAKD-A and PAKD-F under unsteady conditions, and compare the obtained results with the corresponding measurements already performed for PAKB. Section 7.2 will begin by introducing the reduced frequency and flow coefficient parameters. Discussion of the test matrix will follow in Section 7.3. The effect of the varying reduced frequency on the profile losses of the PAKD airfoils and comparison with previous tests done for PAKB will be discussed into greater detail in Section 7.4. Finally, the results will be summarized in the last section.

\subsection{Reduced Frequency and Flow Coefficient}

For the steady flow measurements, such as those described in Chapter 5 and 6 , the similarity criteria are Reynolds number, freestream turbulence intensity and streamwise gradient in the pressure coefficient. To be able to conduct the unsteady flow measurements, in addition to all of the above mentioned parameters, the reduced frequency and flow coefficients should be matched.

Scholz (1977) defined reduced frequency (or the unsteadiness parameter) as the ratio of the total residence time of the wake in the passage to the period between wakes entering the passage. The reduced frequency can be thus related to the number of wakes that are present within the blade passage at any time (the higher the reduced frequency, the more wakes are present in the blade passage). Hence, the definition of the reduced frequency according to Scholz (1977) is as follows: 


$$
f_{\text {Scholz }}=\frac{C_{x} / U_{x i}}{s_{b} / U_{b}}
$$

where $U_{x i}$ is the axial component of inlet velocity and $s_{b}$ is the spacing (pitch) between the bars. $s_{b}$ can be changed only by removing or adding bars. The tangential bar velocity, a function of spoke wheel rotational velocity, is designated by $U_{b}$.

An alternative definition of the reduced frequency, used by Hodson (1985), is given in Eq. 7.2. Hodson used the bar passing frequency, $f_{b}$, normalized by the axial chord and the mass-averaged outlet velocity, $U_{e}$.

$$
f_{\text {Hodson }}=\frac{f_{b} C_{x}}{U_{e}}
$$

where $f_{b}=U_{b} / s_{b}$. Scholz's definition has two advantages over Hodson's. The first advantage is that the reduced frequency directly corresponds to the number of wakes within a blade passage. The second comes from the fact that the reduced frequency in Scholz's definition depends on the inlet axial velocity which is readily available experimentally. This is not the case with Hodson's definition, which uses the massaveraged outlet velocity. That velocity is not known prior to performing the test. Therefore, Scholz's definition will be used for the current measurements. For the sake of simplicity the subscript will be dropped, so that the reduced frequency denoted as $f$ from this point onwards will be the Scholz's definition as given in Eq.7.1.

The second parameter, the flow coefficient, is defined as the ratio of the inlet axial velocity to the bar tangential velocity:

$$
\phi=\frac{U_{x i}}{U_{b}}
$$




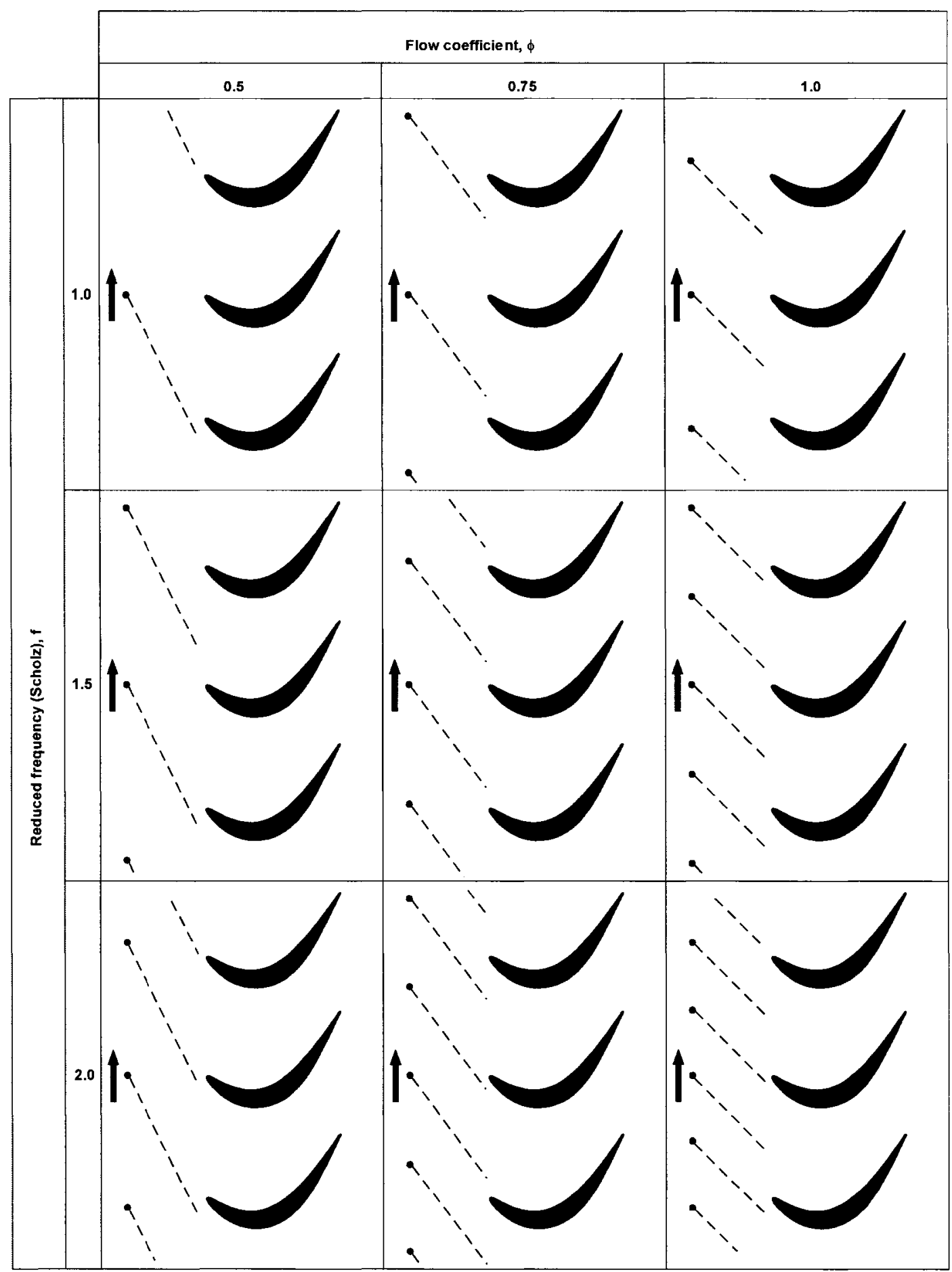

Figure 7.1: Schematic of Wake Trajectories for Varying Flow Conditions (modified from Mahallati, 2003) 
The effect of both flow coefficient and reduced frequency on the wake trajectories can be seen in Figure 7.1. As already mentioned, varying reduced frequency changes the number of the upstream wakes present in the blade passage at any particular time. On the other hand, the flow coefficient influences the wake incidence relative to the blade in the stationary frame of reference; the lower the flow coefficient, the more positive the incidence of the incoming wakes. Mahallati (2003) found that the effect of varying flow coefficient on the measured losses was small compared with the dependence on the reduced frequency. Based on his results, for flow coefficient varied from 0.5 to 1.0 (the range that are typically found in the actual turbine stages) at a given reduced frequency, the average effect on the losses was about $8 \%$. However, a large scatter in the data was observed due the experimental uncertainties. The uncertainty in the profile loss was estimated to be $\pm 1.5 \%$ of mixed-out outlet dynamic pressure, or about $\pm 30 \%$ of the profile loss value. Therefore, the estimated influence of the flow coefficient on losses is minor compared to the overall experimental uncertainties involved with the current experimental set-up and procedures.

Varying both the flow coefficient and the reduced frequency independently is a difficult task, since any change in the spokewheel rotational velocity influences both parameters. The axial velocity, $U_{x i}$, cannot be varied if measurements at constant Reynolds number are desired. The only way to vary one parameter independently from another is through changing the bar spacing (i.e. the number of bars). This is further complicated by the fact that in order to keep the flow periodic and the spokewheel balanced, the spacing between the bars must be kept constant. This limits the possible number of bars installed on the spokewheel.

For the current measurements it was decided to vary the reduced frequency only, and to let the flow coefficient vary so long as it remained between 0.5 and 1.0. From 
above, the resulting variations in losses due to the variations in flow coefficient were within the estimated experimental uncertainties.

\subsection{Test Matrix}

The goal of this part of the study was to examine both PAKD airfoils over the range of reduced frequencies which were considered in the previous measurements for PAKB. Therefore, the measurements were performed for reduced frequencies from 0.5 to 3.0 in increments of about 0.5 . This was achieved by using 20 and 60 bars, as shown in Figure 7.2, while still keeping the flow coefficient in the range of 0.5 to 1.0 .

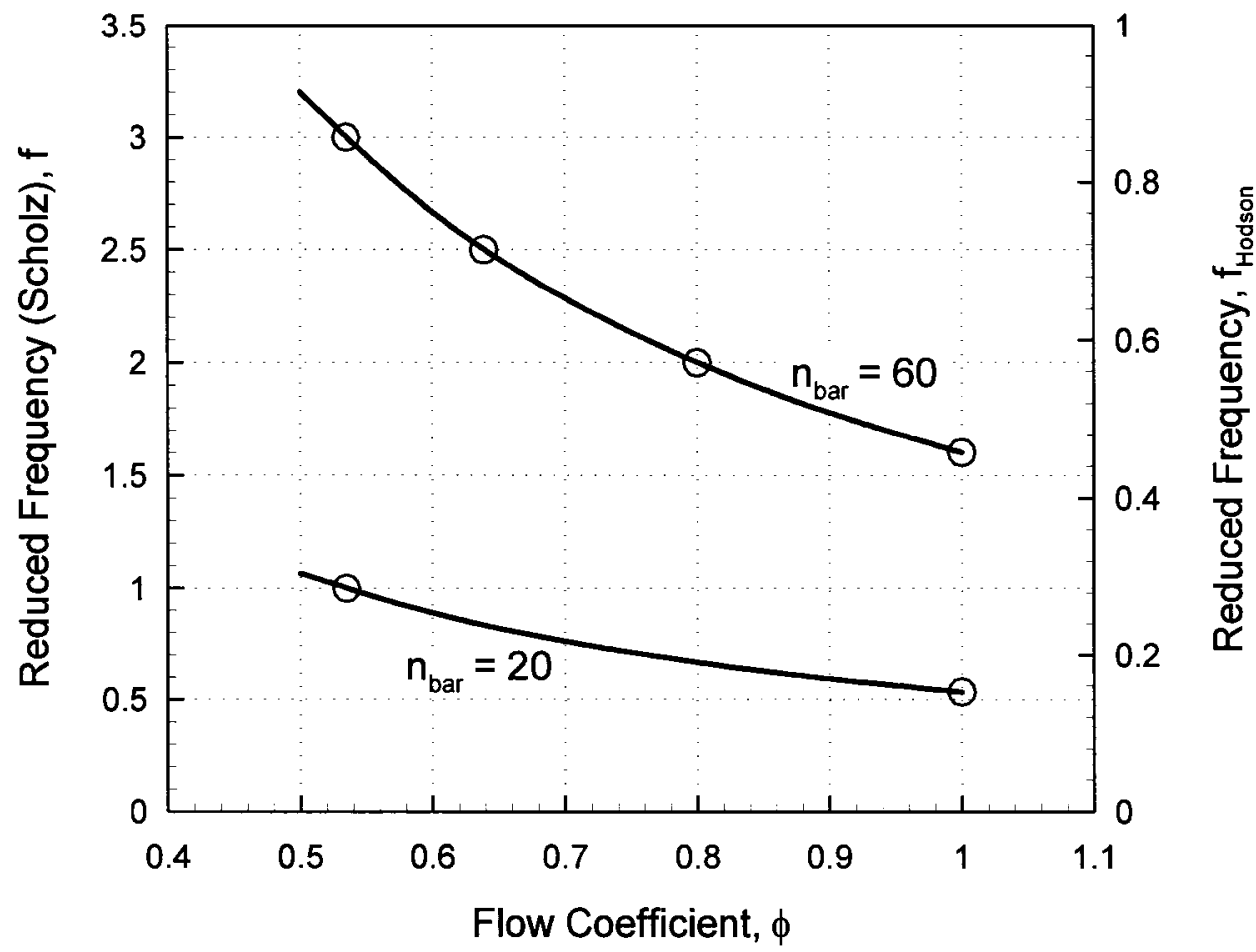

Figure 7.2: Test Matrix for PAKD-A and PAKD-F Airfoils

$$
(R e=50,000, \text { FSTI }=4 \%)
$$


The previous measurements on the PAKB were performed at two FSTI levels: $0.4 \%$ (no grid installed) and $4 \%$ (Grid III). The closely mounted grid for the turbulence generation of $4 \%$ (Grid II) could not be used, since it interferes with the rotating bars. A description of the grids was given in Section 3.2.4.

All present measurements were performed for FSTI of $4 \%$ as generated by Grid III. As in the earlier PAKB measurements, the Reynolds number was also maintained constant at 50,000 (based on inlet velocity and axial chord).

\subsection{Mixed-out Profile Losses}

\subsubsection{The Effect of Varying Reduced Frequency on PAKD-A and PAKD-F}

The variation of profile losses for both PAKD-A and PAKD-F with reduced frequency is shown in Figure 7.3. A description of profile loss calculations for unsteady measurements was provided in Section 4.3. The losses for steady conditions (corresponding to reduced frequency of 0.0 ) are also plotted.

Under steady conditions, at $R e_{i}=50,000$ and FSTI of $4 \%$ with Grid III, the PAKD-A blade is stalled. At the lowest reduced frequency examined (0.5), the loss was substantially reduced compared to that measured under steady conditions indicating that the flow is reattaching. In Chapters 5 and 6 , it was shown that the stall occurs when the flow, after undergoing laminar separation, fails to reattach. The periodic wake passing promotes transition and thus helps the reattaching process and in effect reduces the losses. It is widely accepted that when the reduced frequency 

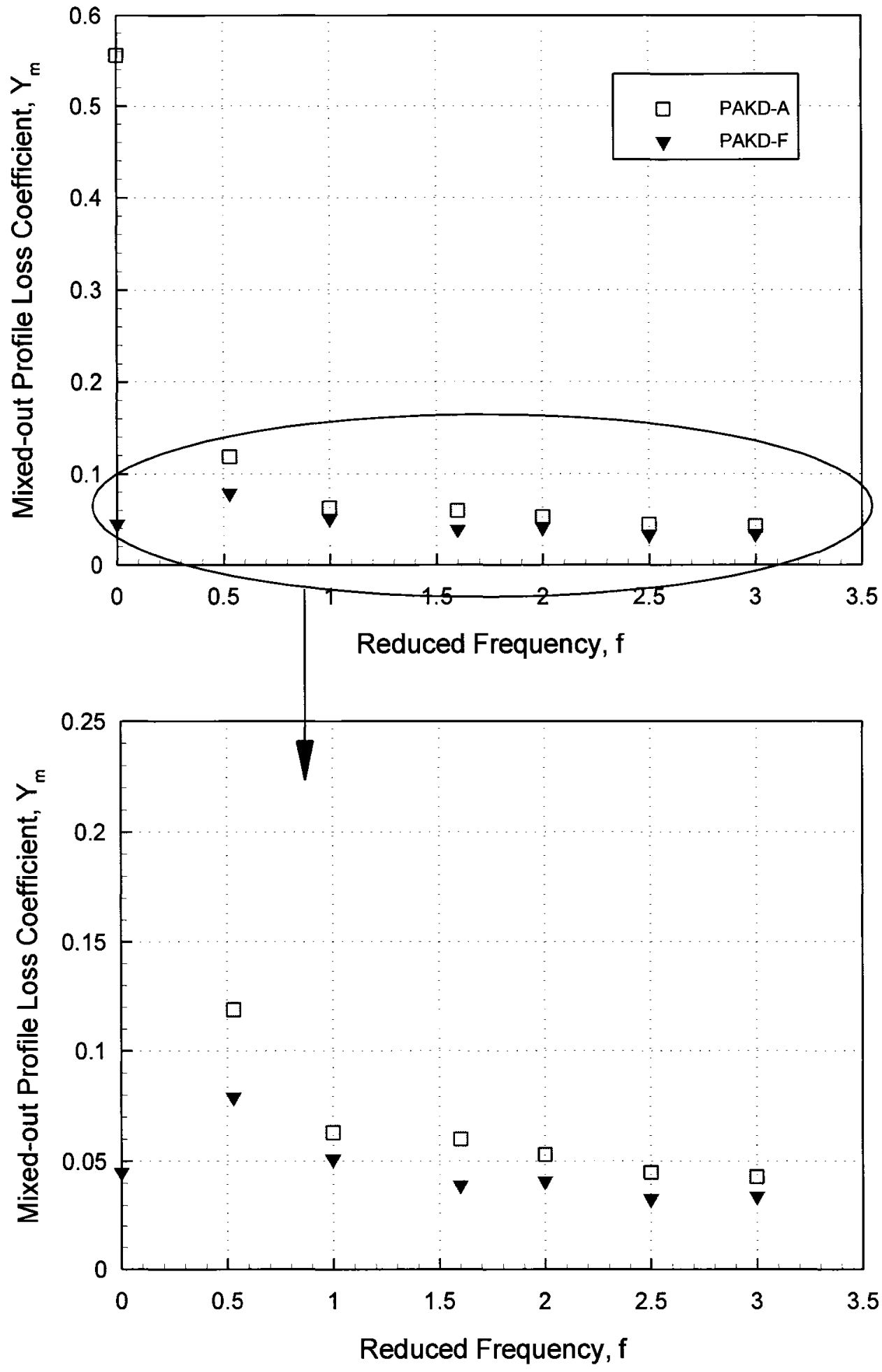

Figure 7.3: Profile Losses for PAKD-A and PAKD-F 
is low, there is sufficient time for the bubble to reestablish itself and grow in size between the two wakes thus increasing the time-averaged losses. Figure 7.4 shows the total blade losses as a function of time for three consecutive wake passing periods at the two lowest reduced frequencies tested for PAKD-A. The dashed lines correspond to the time-averaged total and profile losses. It can be seen that the losses increase between two wake passings. This increase in losses may be attributed, in part, to the bubble growth. Appendix $\mathrm{C}$ provides plots of the instantaneous losses equivalent to Figure 7.4 for both PAKD-A and PAKD-F for the full range of reduced frequencies examined.

At high reduced frequencies, the wake passing is so frequent that the bubble is greatly reduced in size resulting in lower profile losses. It can be concluded that a general trend of loss decrease with increasing reduced frequency for PAKD-A is due to the reduction in the bubble size. Should the frequency continue to be increased, the losses might start rising due to the larger fraction of the surface covered in turbulent boundary layer.

When PAKD-F is subjected to steady flow under these particular conditions (Re of 50,000 and FSTI $=4 \%$ ), the flow remains fully attached, as discussed in Section 5.3.2. In the case of PAKD-F, the unsteady effects result in a loss increase at low reduced frequencies $(f)$, as seen from Figure 7.3. A possible explanation for this phenomenon comes from the fact that the wakes promote temporary transition and thus increase the wetted area covered by the turbulent flow for part of the time. Since turbulent flow generates higher losses than the laminar flow under the same conditions (as discussed in Section 2.5), the time-averaged losses at low reduced frequencies are higher than the steady flow losses. However, it is not clear why the losses for PAKD-F decrease with increased reduced frequency. Orth (1993) studied the effects of periodic 

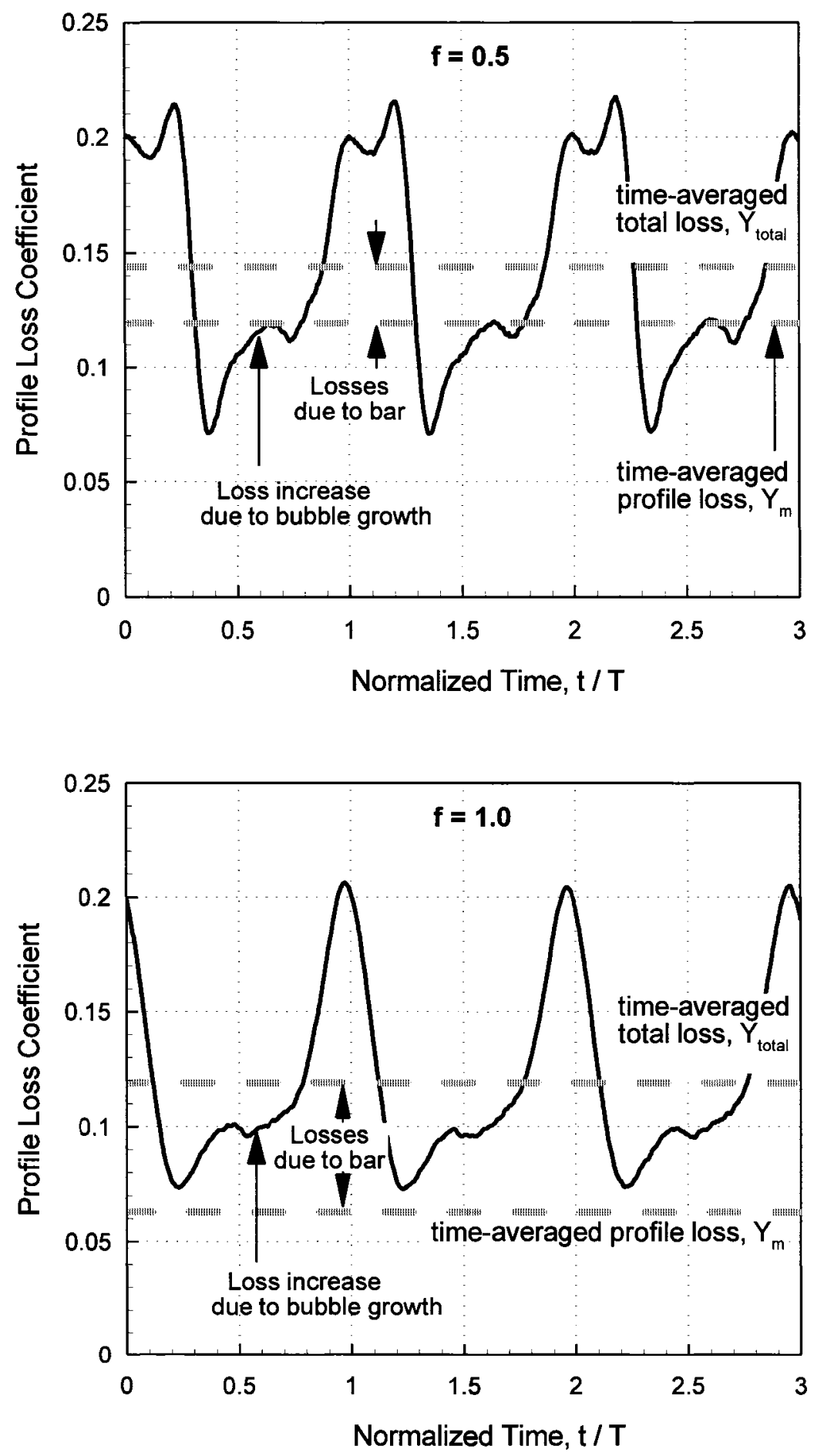

Figure 7.4: Instantaneous Losses for PAKD-A

$$
(\operatorname{Re}=50,000 \text { and } \mathrm{FSTI}=4 \%)
$$


wakes on the transition of the fully attached flow over a flat plate. He has shown that the wakes do promote early onset of transition. However, he also observed that between two wake passings the laminar flow extends downstream far beyond the location of the transition under steady conditions. The picture is thus more complex. The mixed-out profile losses show the combined effect of periodically increased blade wetted area covered by the turbulent flow, followed by the periods of predominantly laminar flow. In the absence of hotfilm measurements, it is not possible to fully understand the transition process occurring on the suction surface.

\subsubsection{Comparison with Previous Measurements for PAKB}

Figure 7.5 shows the profile losses for PAKB measured by Mahallati (2003) compared to the current measurements. The PAKB airfoil is stalled at $R e_{i}$ of 50,000 and FSTI of $0.4 \%$, while at the turbulence intensity of $4 \%$ the separation bubble reattaches before the trailing edge. The unsteady effects are clearly beneficial for the conditions where there is a massive separation under steady flow conditions (PAKB at FSTI of $0.4 \%$ and PAKD-A). For low FSTI the trend for PAKB is thus similar to that for PAKD-A, namely that at $f=0.5$ both airfoils showed a large drop in losses from the values at steady conditions.

At the higher turbulence intensity the losses for PAKB follow a trend similar to that of PAKD-F. For both PAKB and PAKD-F, the losses increase at $f=0.5$ compared with steady conditions. Schulte and Hodson (1998) reported that when a relatively large bubble is present on the suction side of the blade under steady flow, introduction of periodically-unsteady effects will result in reduction of the bubble size as well as the losses. However, their tests at higher Reynolds numbers, when the bubble is either small or non-existent under steady conditions, showed that the 
periodic wake passing in fact increases the overall loss levels, which is consistent with the present observations. However, Schulte and Hodson (1998) did not observe the loss decrease with $f$ increase as was shown in Section 2.6. It should be also noted, that the unsteady measurements have higher experimental uncertainty than the steady measurements, especially at lower reduced frequency (Mahallati, 2003). Therefore, higher than expected losses at $f$ of 0.5 , may also be partly due to the experimental uncertainty.

In section 4.4, it was discussed that the two major sources of uncertainty for the unsteady measurements are the estimation of the bar losses and the assumption that the static pressure at the outlet is constant in space. These uncertainties could be

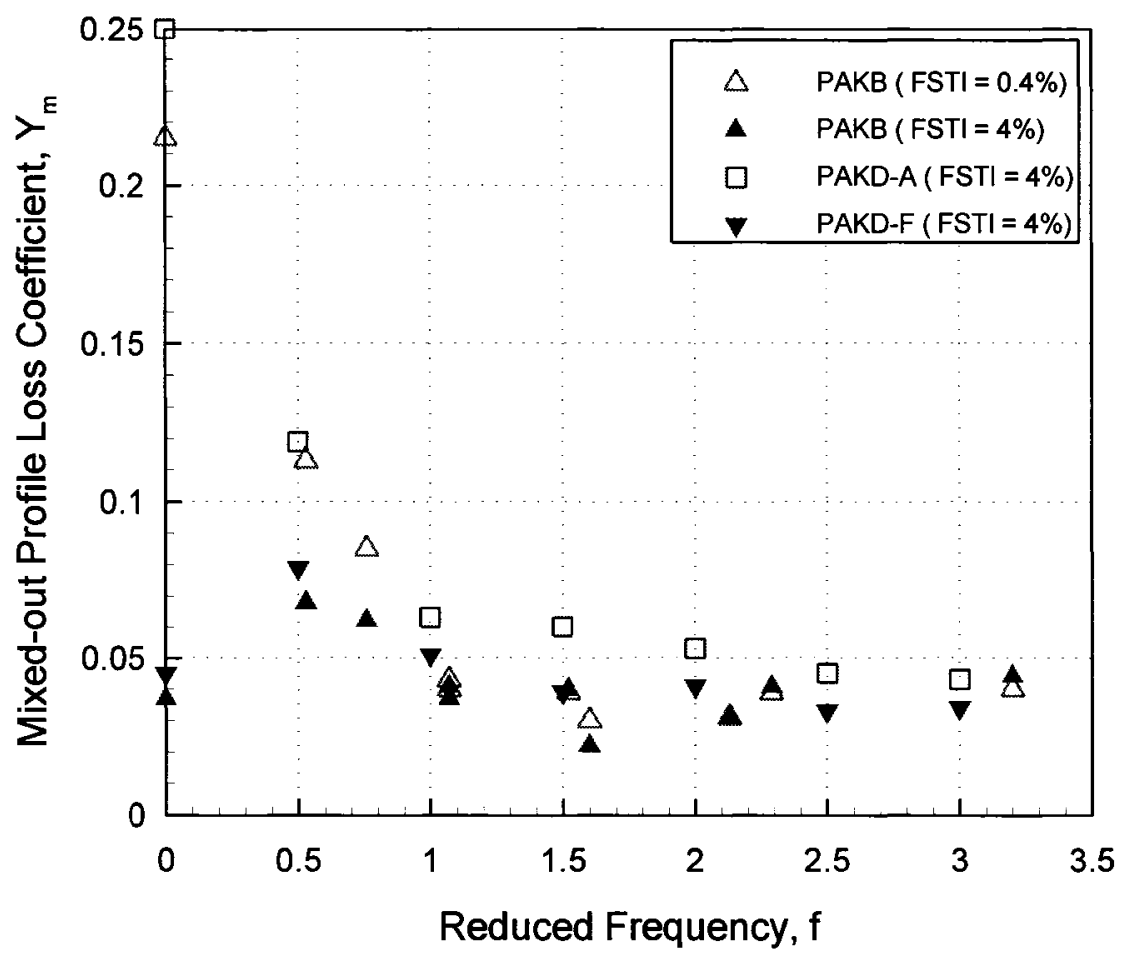

Figure 7.5: Comparison of Profile Losses for all Airfoils

(PAKB data from Mahallati, 2003) 
reduced with use of fast response pressure probes when they become available for measurements at the low levels of dynamic pressure encountered in this experiment. The fast response pressure probes would be able to measure the experimental bar losses and also to infer the static pressure variation at the outlet.

\subsection{Summary of Unsteady Flow Measurements}

This chapter presented the effects of the periodically-unsteady wake passing on the PAKD profile losses. The reduced frequency was varied between 0.5 and 3.0 in steps of about 0.5 at a constant Reynolds number of 50,000 and FSTI of $4 \%$.

It was found that when PAKD-A, which was fully stalled under steady conditions, is subjected to unsteady effects losses decrease by a factor of 5 for the lowest reduced frequency examined $(f=0.5)$. The losses continued to decrease with further increase in reduced frequency. When PAKD-F, fully attached under the steady conditions, was subjected to the periodically-unsteady flow, the losses increased slightly at the reduced frequency of 0.5 . It is believed that the larger blade wetted area covered by turbulent flow. The experimental uncertainty may also be partly responsible for the loss increase at this reduced frequency. However, it was not clear why the losses for PAKD-F continued to decrease with increasing reduced frequency. The current findings were comparable to the previous measurements done on PAKB.

Two major sources of uncertainty in the unsteady measurements were the bar losses and the static pressure assumptions at the outlet plane. A recommendation is to use fast response pressure probes when such probes become available. Such measurements would provide a better estimate of the bar losses and give a more accurate picture of the outlet static pressure variation in time and space. 


\section{Chapter 8}

\section{Conclusions and Recommendations for Future Research}

\subsection{Conclusions}

This thesis presents an experimental study of the loading influence on the performance of a family of three highly-loaded low-pressure turbine blades. All three blades were designed for the same duty: they provide the same flow turning.

The first airfoil investigated was $\mathrm{PAKB}$, which represents the mid-span section of a LP turbine vane designed by Pratt and Whitney Aircraft (PWA), and it is the baseline airfoil for this family. It is an aft-loaded blade inversely redesigned for lowspeed wind tunnel testing. To examine the aerodynamics of turbine blades subjected to higher loading levels, two additional airfoils (designated PAKD-A and PAKD-F) were designed by PWA. The new airfoils were subjected to about $25 \%$ higher loading by increasing the spacing between the blades (pitch). The main difference between 
the two PAKD airfoils is in the distribution of loading. One is aft-loaded (PAKDA), while the other one is front-loaded (PAKD-F). The aerodynamic performance under steady flow conditions was studied over a wide range of Reynolds numbers (from 25,000 to 150,000 based on the inlet velocity and axial chord), two freestream turbulence intensities $(1.5 \%$ and $4 \%)$ and at design incidence and at $-5^{\circ}$ and $+5^{\circ}$ of off-design incidence. In addition, the performance of the two PAKD airfoils under unsteady conditions was investigated at a constant Reynolds number of 50,000 and freestream turbulence intensity of $4.0 \%$ over a range of reduced frequencies. All measurements were performed at midspan, employing airfoil surface pressure taps and pressure probes for the steady flow conditions, and a single-wire hotwire for the unsteady flow conditions. The investigation was divided into three phases.

The first phase of this project focused mainly on the Reynolds number sensitivity and the influence of freestream turbulence intensity on the PAKD-F airfoil at design incidence. The results were compared with those for the other two airfoils, PAKB and PAKD-A, that were previously investigated by Mahallati (2003) and Dai (2004). The tests performed at low Reynolds numbers and freestream turbulence intensity (FSTI) of $1.5 \%$ and $4 \%$ showed that both PAKB and PAKD-F generated comparable losses. This was contradictory to what would have been expected based on traditional loss prediction correlations. However, the traditional correlations apply only to situations where the flow over the blade surface is fully attached, which was not the case for the PAKB airfoil which has a suction side separation bubble present over the whole range of Reynolds numbers investigated at both FSTI levels. In addition, a pressure side separation bubble was also present for all cases with lower FSTI. Since the loss production in the boundary layer varies with $U_{b l}{ }^{3}$, due to higher velocities on the edge of the PAKD-F boundary layer the PAKD-F airfoil was expected to produce higher losses. However, the results show that higher loss production in the PAKD-F 
boundary layer is offset by the fact that PAKB has a separation bubble over the entire range of Reynolds numbers investigated. The separation bubble enhances the local loss production through mixing. The PAKD-A airfoil has higher losses than the other two blades. When compared to PAKB, PAKD-A, similarly to PAKD-F, has higher loss production in the suction side boundary layer. PAKD-A also has a suction side separation bubble present at all investigated conditions, and this again increases its loss production. In addition, PAKD-A stalls at a Reynolds number of about 75,000 - 80,000 at the lower FSTI level, thus increasing the losses significantly. At the higher FSTI level, the stall was delayed until a Reynolds number of about 35,000 to 40,000 . The stall on the PAKD-A airfoil was found to be unsteady for a small range of Reynolds numbers (75,000 - 80,000 for FSTI of $1.5 \%)$. The periodic stall was attributed to the oscillations between the short and long bubble type (also known as "bursting"). However, it is not clear why the long bubble fails to reattach. A possible explanation may be the strong adverse pressure gradient on the aft portion of the airfoil. This topic deserves more attention in the future.

In the second phase of the project all three airfoils were subjected to off-design incidences of $-5^{\circ}$ and $+5^{\circ}$ at constant Reynolds number of 80,000 . The off-design incidence performance is important, since aircraft engines often operate at off-design incidence as well as a range of Reynolds numbers during idle, takeoff, climb etc. The measurements performed at a positive incidence of $+5^{\circ}$ and lower FSTI show higher loss generation for the PAKD-F airfoil when compared to PAKB. It is believed that the higher net loss production at $+5^{\circ}$ incidence for the PAKD-F airfoil is due to the velocity spike and possible separation bubble in the leading edge vicinity. On the other hand, PAKD-A stalled at positive incidence for FSTI of 1.5\% and the losses increased dramatically, thus making the PAKD-A airfoil the worst performer of the blade family at positive incidence and low FSTI. Subjecting the PAKD-A to higher 
FSTI (4\%) resulted in flow reattachment, thus reducing the losses by a factor of about 5. Yet, the losses for PAKD-A remained higher than for the other two blades. The airfoils performance was less sensitive to negative incidence. Although, a separation bubble appeared on the PAKD-F suction surface at negative incidence, it did not affect the loss production significantly. The losses for PAKD-A decreased, but they remained higher than for PAKB and PAKD-F.

The third and the final phase of this project concentrated on unsteady blade row interaction. An upstream blade row was simulated using a wake-generator, with bars generating wake properties representative of an upstream blade row with fully attached flow. Using the same apparatus, PAKB was thoroughly investigated by Mahallati (2003) for a number of different turbulence intensities, Reynolds numbers, flow coefficients and reduced frequencies. The goal of the third phase of the present project was to investigate the effect of varying reduced frequency on the two PAKD airfoils. Measurements were conducted at a Reynolds number of 50,000 and FSTI of $4 \%$ with a variation in reduced frequency from 0.5 to 3.0. PAKD-A, which was stalled under steady flow conditions, greatly benefited from the unsteady effects. A significant reduction of losses was observed even at the lowest reduced frequency examined. The losses continued to decrease with increasing reduced frequency. When PAKD-F, which was fully attached under steady conditions, was subjected to the periodically unsteady flow, the losses increased for the lowest reduced frequency examined. It is believed that the increased wetted area covered by the turbulent flow may be responsible for this increase in losses. However, it was not clear why the losses for PAKD-F continued to decrease with increasing reduced frequency.

Empirical loss correlations are still widely used in preliminary turbine design. Therefore, the present results were used to evaluate some of them. Two design 
incidence correlations, Kacker and Okapuu (1982) and Zhu and Sjolander (2005), were compared with the experimental results. It was found that the Zhu-Sjolander (Z-S) correlation provides a better agreement with the experimental data than Kacker-Okapuu's. However, it should be noted that part of the current data set was used to develop the Z-S correlation. The off-design loss correlations examined here, Moustapha et al. (1990) and Benner et al. (1997), use traditional geometrical parameters such as leading edge circle and inlet wedge angle to correlate the off-design performance of the blades. The main difficulty in applying these correlations, and perhaps the reason why they predicted the losses poorly, was that the current blades use neither leading edge circles nor inlet wedge angles, as assumed in the correlations. This indicated a need to develop alternative correlations that are more applicable to modern airfoil shapes.

Based on the measurements it can be concluded that a $25 \%$ increase in loading (relative to the baseline airfoil) is possible with almost no penalty in losses by employing the PAKD-F airfoil. The PAKD-F airfoil does not stall under any conditions investigated, and there appears to be no separation bubble present on the suction side of the airfoil except at $-5^{\circ}$ incidence and the lowest Reynolds numbers examined at design incidence. These results are encouraging for turbine designers, since so far it has been widely accepted that separation bubbles are almost unavoidable in highly-loaded low-pressure turbines. This shows that CFD based design tools have evolved to the degree that they can help expand the design envelope. However, the present study has covered only one loss component, profile losses. A complete comparison between the three blades is not possible until the secondary losses are also investigated. At the time of preparation of this manuscript, a separate study on the secondary flows of these blades is being conducted by Zoric (2006). Also, a number of questions concerning the midspan behavior of these blades remain 
unanswered. Hence this study can represent the foundation for further research concerning this blade family. Suggestions for possible directions of such investigation will be the topic of the next section.

\subsection{Recommendations for Future Research}

The results of the present study have posed questions regarding several areas that merit further attention.

First, the bubble "bursting" on the suction surface of PAKD-A airfoil was shown to be a highly unsteady phenomenon, but the source of the instability was not identified. However, it was demonstrated that the "bursting" is a function of the pressure gradients, FSTI and Reynolds number. Measurements by Murawski and Vafai (1999) have shown that the stall on the PAKB airfoil can be delayed by extending the length of the suction surface (increasing the axial chord) and allowing the bubble to reattach. Similar measurements might be attempted on PAKD-A to investigate whether the flow would reattach for low Reynolds numbers. This would answer the question of whether a long-type bubble is possible on the suction surface of the PAKD-A airfoil, since the bubble that was identified at higher Reynolds number was of the short type.

Secondly, it would be interesting to investigate the transition process from laminar to turbulent flow on the suction side of the PAKD-F airfoil, since it is closely related to an understanding of its good tolerance to the wide range of conditions examined. In order to investigate the transition process either hotfilm array should be instrumented on one of the blades, or at least flow visualization should be performed.

Furthermore, the unsteady measurements are subjected to high experimental 
uncertainty. The major sources of the uncertainty are the estimation of the bar losses and the assumption of the constant outlet static pressure. However, with the current work on the development of the fast-response pressure probes capable of measuring low levels of dynamic pressure, it may be possible to measure the unsteady bar losses and the total pressure losses directly in the future, thus reducing the uncertainties involved.

The measurements performed over the course of this investigation have opened the door for even more aggressive blade designs in terms of loading. Although the author is fully aware that the process of increasing the blade loading cannot go on indefinitely, it is obvious from this study that the current loading levels do not represent the upper limit. The present study has shown that blades with loadings comparable to PAKDF can be utilized without employing the flow control and/or periodic wake passing. It was also shown that PAKD-A benefited greatly from the unsteady effects at low Reynolds number. However, blades with even higher loading levels might require some form of flow control, perhaps in addition to the periodic-wake passing. This opens the possibility of attempting to synchronize the wake passing frequency with flow control, perhaps in the form of pulsed air injection, as this might reduce the amount of air necessary for flow control and at the same time help to achieve substantially higher loading levels. 


\section{References}

Ainley, D. G. and Mathieson, G. C. R. (1951), "A Method of Performance Estimation for Axial Flow Turbines," British ARC R\&M, 2974.

Arndt, N. (1993), "Blade Row Interaction in a Multistage Low-Pressure Turbine," Journal of Turbomachinery, 115, pp. 137-146.

Bearman, P. W. (1971), "Corrections for the Effect of Ambient Temperature Drift on Hotwire Measurements in Incompressible Flow," DISA Information, 11, pp. 2530.

Benner, M. W. (2003), "The Effects of Leading Edge Geometry on Profile and Secondary Losses in Turbine Cascades," Ph.D. thesis, Carleton University, Ottawa, Canada.

Benner, M. W., Sjolander, S. A. and Moustapha, S. H. (1997), "Influence of Leading-Edge Geometry on Profile Losses in Turbines at Off-Design Incidence: Experimental Results and Improved Correlation," Journal of Turbomachinery, 119, pp. 193-200.

Brear, M. J. and Hodson, H. P. (2004), "The effect of Wake Passing on a Flow Separation in a Low-Pressure Turbine Cascade," Journal of Turbomachinery, 126, pp. 393-256. 
Brear, M. J., Hodson, H. P. and Harvey, N. W. (2002), "Pressure Surface Separations in Low-Pressure Turbines-Part 1: Midspan Behavior," Journal of Turbomachinery, 124, pp. 393-401.

Brunn, H. H. (1995), Hotwire Anemometry: Principles and Signal Analysis, 1st ed., Oxford University Press, New York.

Dai, W. (2004), "Steady Aerodynamic Performance of a Very Highly-Loaded LowPressure Turbine Airfoil," Master's thesis, Carleton University, Ottawa, Canada.

Demeulenare, W. N. and den Brembussche, R. V. (1996), "Three-Dimensional Inverse Method for Turbomachinery Blading Design," ASME paper No. 96-GT-39.

Denton, J. D. (1993), "Loss Mechanisms in Turbomachines," Journal of Turbomachinery, 115, pp. 621-652.

Dunham, J. and Came, P. M. (1970), "Improvments to the Ainley/Mathieson Method of Turbine Performance Predictions," Journal of Engineering for Gas Turbine and Power, pp. 252-256.

Gaster, M. (1967), "The Structure and Behaviour of Separation Bubbles," ARC R\&M No. 3395 .

Gonzáles, P., Ulizar, I. and Vázquez, R. (2001), "Pressure and Suction Surfaces Redesign for High Lift Low Pressure Turbines," ASME 2001-GT-0439, New Orleans, Louisiana.

Goobie, S. M. (1989), "A Test Section for Investigating the Off-Design Performance of Planar Cascades," Ph.D. thesis, Carleton University, Ottawa, Canada.

Hinze, J. O. (1975), Turbulence, 2nd ed., McGraw-Hill, New York. 
Hodson, H. P. (1985), "Measurements of Wake-Generated Unsteadiness in the Rotor Passage of Axial Flow Turbines," Journal of Engineering for Gas Turbine and Power, 107, pp. 467-476.

Hodson, H. P. and Dawes, W. N. (1998), "On the Interpreration of Measured Profile Losses in Unsteady Wake-Turbine Blade Interaction Studies," Journal of Turbomachinery, 120, pp. 276-284.

Hodson, H. P. and Dominy, R. G. (1987), "The Off-Design Performance of a LowPressure Turbine Cascade," Journal of Turbomachinery, 109, pp. 201-209.

Hodson, H. and Steele, I. H. A. (1994), "An Investigation of Boundary Layer Develop,emt in a Multistage LP Turbine," Journal of Turbomachinery, 116, pp. 375.

Hoheisel, H., Kiock, R., Lichtfuss, H. J. and Fottner, L. (1987), "Influence of FreeStream Turbulence and Blade Pressure Gradient on Boundary Layer and Loss Behaviour of Turbine Cascade," Journal of Turbomachinery, 109, pp. 210-219.

Hollon, B. and Jacob, J. (2001), "Experimental Investigation of Separation on Low Pressure Turbine Blades," AIAA 2001-0447, Reno, Nevada.

Houtermans, R., Coton, T. and Arts, T. (2004), "Aerodynamic Performance of a Very High Lift Low Pressure Turbine Blade With Emphasis on Separation Prediction," Journal of Turbomachinery, 126, pp. 406-413.

Howell, R. J., Ramesh, O. N., Hodson, H. P., Harvey, N. W. and Schulte, V. (2001), "High Lift and Aft-Loaded Profiles for Low-Pressure Turbines," Journal of Turbomachinery, 123, pp. 181-188. 
Islam, A. M. T. and Sjolander, S. A. (1999), "Deviation in Axial Turbines at Subsonic Conditions," ASME paper No. 99-GT-026, Indianapolis, IN.

Kacker, S. and Okapuu, U. (1982), "A Mean Line Prediction Method for Axial Flow Turbine Efficiency," Journal of Engineering for Power, 104, pp. 111-119.

Korakianitis, T. and Papagiannidis, P. (1993), "Surface-Curvature-Distribution Effects on Turbine-Cascade Performance," Journal of Turbomachinery, 115, pp. $334-340$.

Lake, J. P., King, P. and Rivir, R. B. (1999), "Effects of Turbulence and Solidity on the Boundary Layer Development in a Low-Pressure Turbine," AIAA 99-0242, Reno, Nevada.

Langtry, R. B. (2002), "Prediction of Transition for Attached and Separated Shear Layers in Turbomachinery," Master's thesis, Carleton University, Ottawa, Canada.

Lewis, A. B. (1966), "Fixed-Direction Probes for Aerodynamic Measurements," Proc. Inst. Mech. Engrs, Pt.3J, 180, pp. 141-152.

Lou, W. and Hourmouziadis, J. (2000), "Separation Bubbles Under Steady and Periodic-Unsteady Main Flow Conditions," Journal of Turbomachinery, 122, pp. 634-643.

Mahallati, A. (2003), "Aerodynamics of a Low-Pressure Turbine Airfoil Under Steady and Periodically Unsteady Conditions," Ph.D. thesis, Carleton University, Ottawa, Canada.

Mayle, R. E. (1991), "The Role of Laminar-Turbulent Transition in Gas Turbine Engines," Journal of Turbomachinery, 113, pp. 509-531. 
McAuliffe, B. (2003), "An Experimental Study of Flow Control Using Blowing for a Low-Pressure Turbine Airfoil," Master's thesis, Carleton University, Ottawa, Canada.

Moffat, R. J. (1982), "Contributions to the Theory of Single-Sample Uncertainty," Journal of Fluid Engineering, 104, pp. 250-260.

Moustapha, S. H., Kacker, S. C. and Tremblay, B. (1990), "An Improved Incidence Losses Prediction Method for Turbine Airfoils," Journal of Engineering for Power, 112, pp. 267-276.

Murawski, C. G. and Wafai, K. (1999), "Effects of Incidence on Three-Dimensional Flows in a Linear Turbine Cascade," Journal of Propulsion and Power, 15, pp. $667-674$.

Orth, U. (1993), "Unsteady Boundary-Layer Transition in Flow Periodically Disturbed by Wakes," Journal of Turbomachinery, 115, pp. 707-713.

Qui, S. and Simon, T. W. (1997), "An Experimental Investigation of Transiton as Applied to Low-Pressure Turbine Suction Surface Flows," ASME 97-GT-455.

Roach, P. E. (1987), "The Generation of Nearly Isotropic Turbulence by Means of Grids," International Journal of Heat and Fluid Flow, 8, pp. 82-92.

Rodger, P. W. (1992), "Establishing Two-Dimensional Flow in a Large Scale Planar Turbine Cascade," Master's thesis, Carleton University, Ottawa, Canada.

Schlichting, H. and Gersten, K. (2000), Boundary-Layer Theory, 8th ed., SpringerVerlag, Berlin.

Scholz, N. (1977), "Aerodynamics of Cascade," AGARD-AG-220. 
Schulte, V. and Hodson, H. P. (1998), "Unsteady Wake-Induced Boundary Layer Transition in High Lift LP Turbine," Journal of Turbomachinery, 120, pp. 2835.

Shyne, R. J., Sohn, K. H. and DeWitt, K. J. (2000), "Experimental Investigation of Boundary Layer Behavior in a Simulated Low-Pressure Turbine," Journal of Fluids Engineering, 122, pp. 84-89.

Solomon, W. J. (2000), "Effects of Turbulence and Solidity on the Boundary Layer Development in a Low-Pressure Turbine," ASME 2000-GT-0273, Munich, Germany.

Tremblay, B. (1990), "Measurement and Correlation of Off-Design Performance of Axial-Flow Turbines," Master's thesis, Carleton University, Ottawa, Canada.

Treuren, K. W. V., Simon, T. and von K M. (n.d.).

Vijayaraghavan, S. B. and Kavanagh, P. (1988), "Effects of Free-Stream Turbulence, Reynolds Number, and Incidence on Axial Turbine Cascade Performance," ASME 88-GT-152, Amsterdam, The Netherlands.

Volino, R. V. and Hultgren, L. S. (2001), "Measurements in Separated and Transitional Boundary Layers Under Low-Pressure Turbine Airfoil Conditions," Journal of Turbomachinery, 123, pp. 189-197.

Walker, G. J. (1993), "The Role of Laminar-Turbulent Transition in Gas Turbine Engines: A Discussion," Journal of Turbomachinery, pp. 207-217.

Walraevens, R. E. and Clumpsty, N. A. (1995), "Leading Edge Separation Bubbles on Turbomachinery Blades," Journal of Turbomachinery, 117, pp. 115-125. 
Whitehouse, D. R., Moustapha, S. H. and Sjolander, S. A. (1993), "The Effect of Axial Velocity Ratio, Turbulence Intensity, Incidence, and Leading Edge Geometry on the Midspan Performance of Turbine Cascade," Canadian Aeronautics and Space Journal, 39.

Yamamoto, A. and Nouse, H. (1988), "Effects of Incidence on Three-Dimensional Flows in a Linear Turbine Cascade," Journal of Turbomachinery, 110, pp. 486 496.

Zavitz, K. and Sjolander, S. (2001), "Design of Low-Speed Cascades for Investigating Viscous Effects in High-Speed Axial Turbines," Journal of Turbomachinery, 123, pp. $181-188$.

Zhang, X. F. (2002), "Hot-Film Measurements of the Steady and Unsteady Boundary Layer Development on a Low-Pressure Turbine Airfoil," Master's thesis, Carleton University, Ottawa, Canada.

Zhang, X. F., Vera, M. and Hodson, H. P. (2005), "Separation and Transition Control on an Aft-loaded Ultra-High-Lift LP Turbine Blade at Low Reynolds Numbers: Low-Speed Investigation," ASME 2005-GT-68892, Reno, Nevada.

Zhu, J. and Sjolander, S. A. (2005), "Improved Profile Loss and Deviation Correlations for Axial-Turbine Blade Rows," ASME paper No. GT2005-69077, Reno, NE.

Zorić, T. (2006), "Comparative Study of Secondary Losses 3 Highly-Loaded LowPressure Turbine Cascades," Master's thesis, Carleton University, Ottawa, Canada.

Zweifel, O. (1945), "The Spacing of Turbo-Machine Blading Especially with Large Angular Deflection," The Brown Boveri Review, pp. 436-444. 


\section{Appendix A}

\section{Static Pressure Tap Locations}

This appendix presents blades that were instrumented with the pressure taps for loading measurements. In the case of PAKB airfoil both suction and pressure side holes were instrumented on the same blade (presented in Figure A.1) by Mahallati (2003).

Figures A.2 and A.3 show PAKD-A and PAKD-F blades respectively. As can be seen from the figures, the suction side taps are located on the middle blade, while the adjacent blade accommodates the taps on the pressure side.

The reason for this change was of practical nature. In case of PAKB airfoil, due to very small blade thickness near the trailing edge, some of the taps shared the same internal tubing. Therefore, it was not possible to take the blade loading without an interruption. (While one side was measured, the other was sealed in order to prevent air leakage).

In case of PAKD airfoils, this problem was eliminated by using two loading blades. Due to both periodicity of the outlet flow and the uniformity of the inlet flow, all 
blades have virtually the same loading with the exception of the blades at the very end of the cascade. Therefore, it is believed that this strategy did not introduce any additional uncertainties in the loading measurements.

The non-dimensional axial and surface length locations of the pressure taps are tabulated in Table A.1.

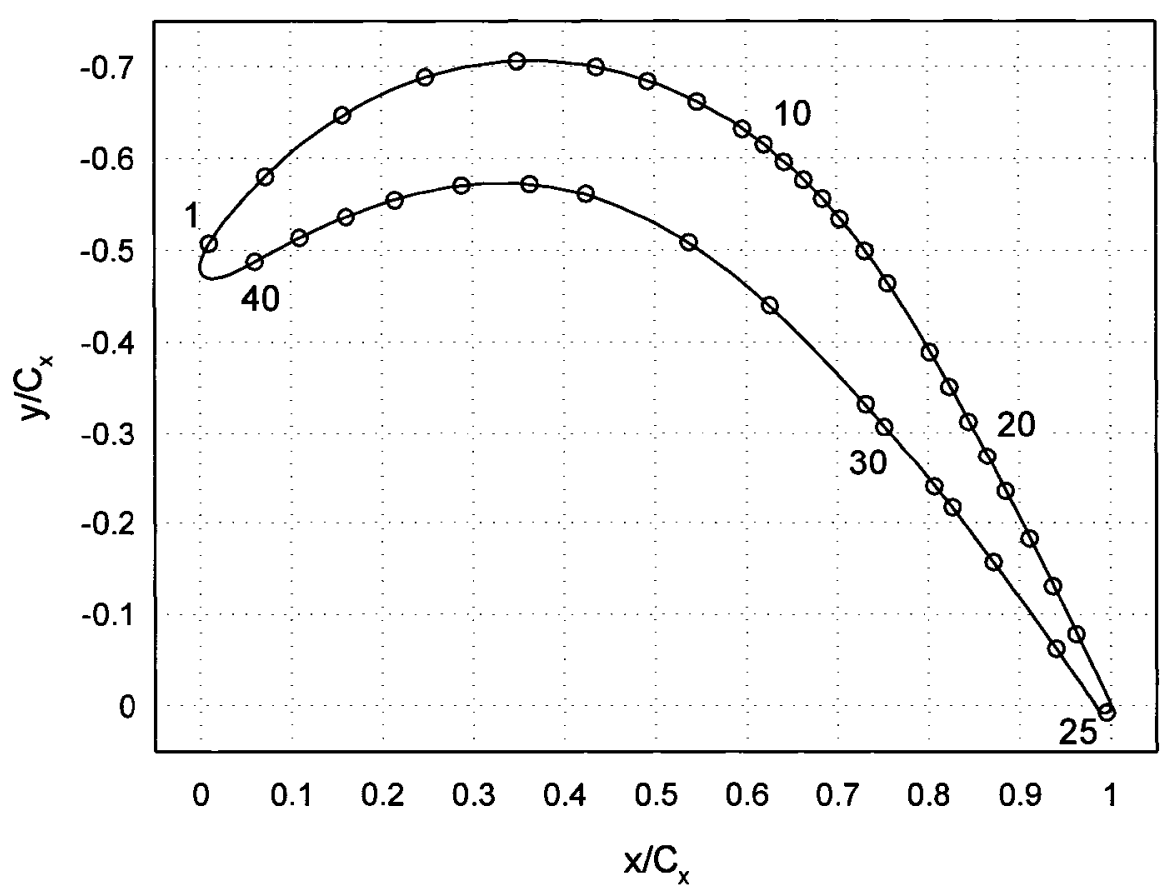

Figure A.1: Surface Static Pressure Taps on PAKB 


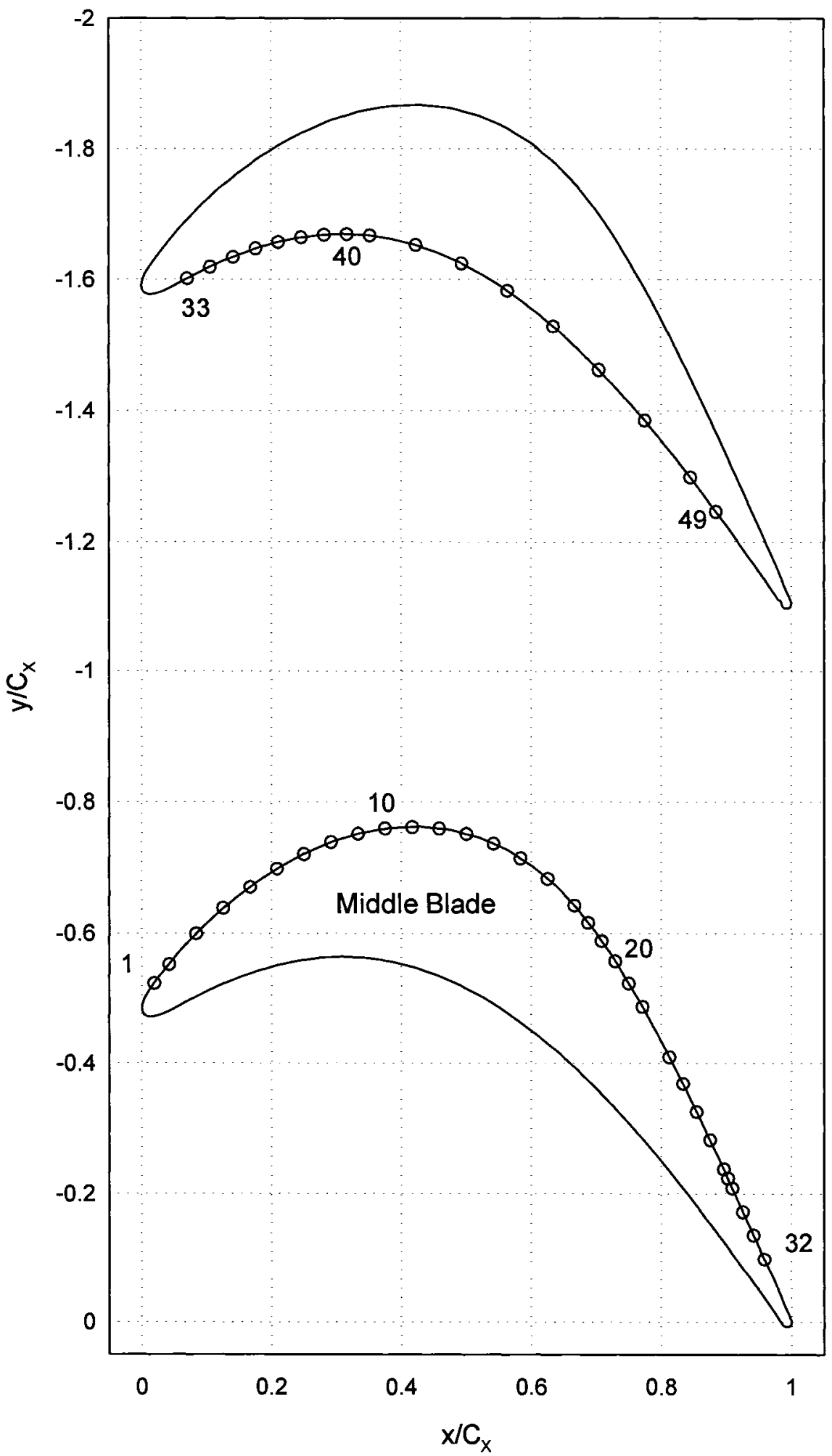

Figure A.2: Surface Static Pressure Taps on PAKD-A 


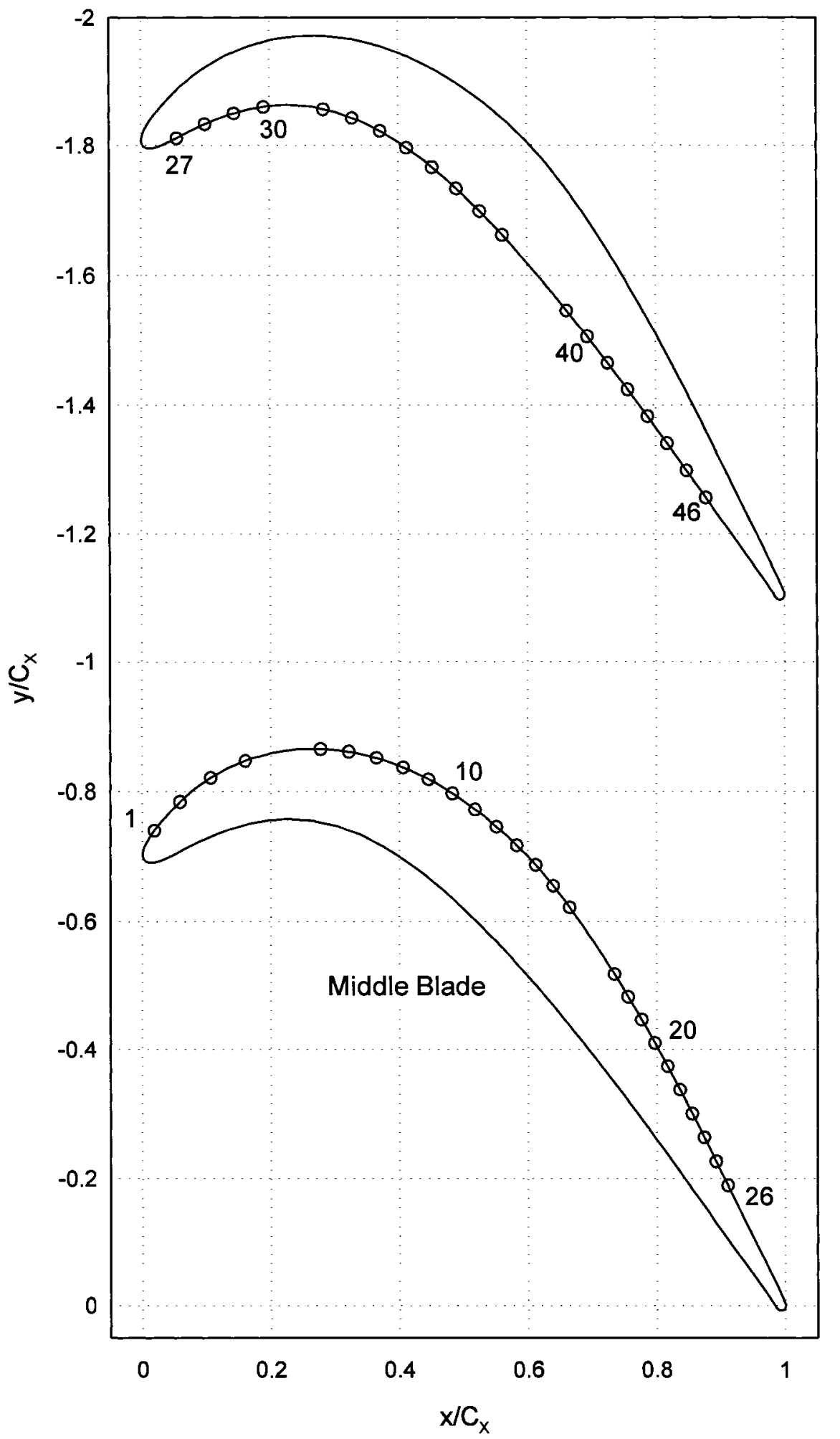

Figure A.3: Surface Static Pressure Taps on PAKD-F 
Table A.1: Coordinates of Pressure Taps

\begin{tabular}{|c|c|c|c|c|c|c|}
\hline & \multicolumn{2}{|c|}{ PAKB } & \multicolumn{2}{|c|}{ PAKD-A } & \multicolumn{2}{|c|}{ PAKD-F } \\
\hline & $\mathbf{X} / \mathbf{C}_{\mathbf{X}}$ & $\overline{\text { SLF }}$ & $\mathbf{X} / \mathbf{C}_{\mathbf{X}}$ & SLF & $\mathbf{X} / \mathbf{C}_{\mathbf{X}}$ & SLF \\
\hline 1 & 0.0080 & 0.0225 & 0.0190 & 0.0287 & 0.0183 & 0.0276 \\
\hline 2 & 0.0717 & 0.0898 & 0.0417 & 0.0527 & 0.0582 & 0.0667 \\
\hline 3 & 0.1554 & 0.1617 & 0.0833 & 0.0933 & 0.1059 & 0.1063 \\
\hline 4 & 0.2484 & 0.2314 & 0.1250 & 0.1304 & 0.1600 & 0.1459 \\
\hline 5 & 0.3478 & 0.2999 & 0.1667 & 0.1649 & 0.2773 & 0.2245 \\
\hline 6 & 0.4353 & 0.3595 & 0.2084 & 0.1975 & 0.3214 & 0.2536 \\
\hline 7 & 0.4919 & 0.3992 & 0.2500 & 0.2284 & 0.3644 & 0.2826 \\
\hline 8 & 0.5461 & 0.4389 & 0.2917 & 0.2579 & 0.4057 & 0.3115 \\
\hline 9 & 0.5962 & 0.4787 & 0.3334 & 0.2863 & 0.4449 & 0.3400 \\
\hline 10 & 0.6203 & 0.4985 & 0.3750 & 0.3139 & 0.4820 & 0.3683 \\
\hline 11 & 0.6425 & 0.5183 & 0.4167 & 0.3412 & 0.5171 & 0.3964 \\
\hline 12 & 0.6641 & 0.5382 & 0.4584 & 0.3684 & 0.5504 & 0.4244 \\
\hline 13 & 0.6848 & 0.5580 & 0.5000 & 0.3962 & 0.5819 & 0.4524 \\
\hline 14 & 0.7039 & 0.5779 & 0.5417 & 0.4251 & 0.6115 & 0.4804 \\
\hline 15 & 0.7312 & 0.6077 & 0.5834 & 0.4559 & 0.6390 & 0.5082 \\
\hline 16 & 0.7564 & 0.6375 & 0.6251 & 0.4898 & 0.6647 & 0.5358 \\
\hline 17 & 0.8134 & 0.7110 & 0.6667 & 0.5280 & 0.7339 & 0.6179 \\
\hline 18 & 0.8248 & 0.7269 & 0.6876 & 0.5495 & 0.7553 & 0.6452 \\
\hline 19 & 0.8450 & 0.7554 & 0.7084 & 0.5724 & 0.7762 & 0.6725 \\
\hline 20 & 0.8664 & 0.7865 & 0.7292 & 0.5967 & 0.7965 & 0.6998 \\
\hline 21 & 0.8854 & 0.8147 & 0.7501 & 0.6225 & 0.8163 & 0.7270 \\
\hline 22 & 0.9128 & 0.8559 & 0.7709 & 0.6497 & 0.8357 & 0.7542 \\
\hline 23 & 0.9375 & 0.8937 & 0.8126 & 0.7072 & 0.8548 & 0.7814 \\
\hline 24 & 0.9644 & 0.9371 & 0.8334 & 0.7373 & 0.8735 & 0.8086 \\
\hline 25 & 0.9951 & 1.0000 & 0.8542 & 0.7683 & 0.8920 & 0.8358 \\
\hline 26 & 0.9409 & 0.9265 & 0.8751 & 0.7999 & 0.9102 & 0.8630 \\
\hline 27 & 0.8724 & 0.8338 & 0.8959 & 0.8320 & 0.0552 & 0.0481 \\
\hline 28 & 0.8265 & 0.7731 & 0.9025 & 0.8423 & 0.0990 & 0.0837 \\
\hline 29 & 0.8086 & 0.7512 & 0.9091 & 0.8526 & 0.1442 & 0.1189 \\
\hline 30 & 0.7529 & 0.6815 & 0.9254 & 0.8783 & 0.1905 & 0.1535 \\
\hline 31 & 0.7311 & 0.6543 & 0.9418 & 0.9042 & 0.2834 & 0.2216 \\
\hline 32 & 0.6263 & 0.5362 & 0.9584 & 0.9307 & 0.3284 & 0.2560 \\
\hline 33 & 0.5369 & 0.4468 & 0.0704 & 0.0657 & 0.3716 & 0.2908 \\
\hline 34 & 0.4236 & 0.3479 & 0.1057 & 0.0967 & 0.4128 & 0.3264 \\
\hline 35 & 0.3616 & 0.2981 & 0.1409 & 0.1268 & 0.4521 & 0.3624 \\
\hline 36 & 0.2877 & 0.2396 & 0.1761 & 0.1563 & 0.4898 & 0.3989 \\
\hline 37 & 0.2129 & 0.1790 & 0.2113 & 0.1851 & 0.5261 & 0.4355 \\
\hline 38 & 0.1592 & 0.1341 & 0.2465 & 0.2133 & 0.5611 & $0 . \overline{4724}$ \\
\hline 39 & 0.1076 & 0.0893 & 0.2818 & 0.2412 & 0.6611 & 0.5840 \\
\hline 40 & 0.0577 & 0.0447 & 0.3170 & 0.2690 & 0.6932 & 0.6214 \\
\hline 41 & & & 0.3522 & 0.2967 & 0.7247 & 0.6589 \\
\hline 42 & & & 0.4226 & 0.3534 & 0.7559 & 0.6963 \\
\hline 43 & & & 0.4931 & 0.4130 & 0.7867 & 0.7338 \\
\hline 44 & & & 0.5635 & 0.4774 & 0.8172 & 0.7714 \\
\hline 45 & & & 0.6340 & 0.5475 & 0.8474 & 0.8090 \\
\hline 46 & & & 0.7044 & 0.6235 & 0.8775 & 0.8466 \\
\hline 47 & & & 0.7863 & 0.7281 & & \\
\hline 48 & & & 0.8682 & 0.8413 & & \\
\hline 49 & & & 0.9501 & 0.9217 & & \\
\hline
\end{tabular}




\section{Appendix B}

\section{Discussion of Static Pressure Coefficients for Loading Plots}

This appendix presents a discussion of the two definitions of the pressure coefficients for loading plots that have been used in the thesis. The first coefficient, used for all the loading measurements at design incidence presented in Chapter 5 , is normalized by the inlet dynamic pressure and is given by:

$$
C_{P l Q i}=\frac{P_{0 i}^{\prime}-P_{S l}}{P_{0 i}^{\prime}-P_{S i}^{\prime}}
$$

The second coefficient, used for all the loading measurements at off-design incidence presented in Chapter 6 , is normalized by the outlet dynamic pressure and can be calculated from:

$$
C_{P l Q e}=\frac{P_{0 i}^{\prime}-P_{S l}}{P_{0 e}^{\prime}-P_{S e}^{\prime}}
$$


Both definitions of the pressure coefficients are valid; that is, both are nondimensionalized by reference quantities obtained from the particular flow. The advantage of the first definition lies in the fact that it is referenced to inlet values, which are readily available at the time when the measurements are taken. The second definition, however, is referenced on the outlet values of the dynamic pressure, which can be obtained only after detailed downstream measurements and data reduction are performed.

Use of the inlet reference pressure coefficient is useful in cases when Reynolds number is varied over a wide range of values for a single airfoil, as was the case in Chapter 5 of the thesis. Figure B.1 shows the loading for PAKD-F at design incidence and Reynolds numbers varying from 25,000 to 150,000 .

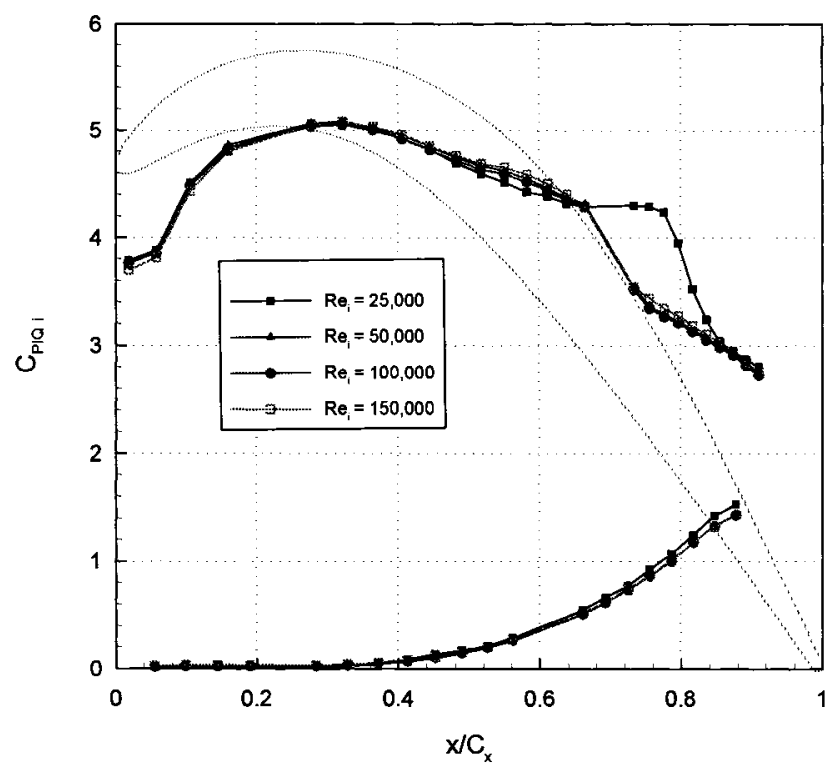

Figure B.1: PAKD-F: Loading Distribution at Design Incidence and FSTI = $1.5 \%$

Clearly, the choice of inlet-based pressure coefficients gives a good visual represen- 
tation of the flow changes over the blade surfaces, such as the distributions of blade loading, and the appearance of a separation bubble at low Reynolds numbers.

The choice of inlet-based pressure coefficient at off-design conditions, such as those shown in Chapter 6, and in Figure B.2 is less satisfactory. Intuitively one would expect that with increasing incidence the loading will increase too. However, Figure B.2(a) shows the opposite - an apparent increase in loading with the decrease in incidence. The plot in Figure B.2(b), which used the outlet-based pressure coefficient, visually confirms that the loading will increase with increasing incidence. Based on Figure B.2 it is clear that loadings at off-design incidence will give a better visual representation if they are referenced to outlet dynamic pressure. This loading definition is also directly related to the definition of the Zweifel coefficient $\left(Z_{w}\right)$. The Zweifel coefficient (explained in Section 2.3) is very often used as a measure of turbine blade loading.

At off-design conditions, the velocity triangles are changed due to increased or decreased incidence and also by the changes in the outlet flow deviation. At positive incidence, as defined in Section 2.1, the flow turning is increased and hence the blade is exerting a larger tangential force. Therefore, the Zweifel coefficient increases with a positive incidence and vice versa, as shown in Figure B.3. It will be demonstrated that the area enclosed in the loading plot which uses $C_{P l Q}$, directly corresponds to the variation in Zweifel.

In Section 2.3, the Zweifel was defined as:

$$
Z_{w}=\frac{F_{Y}}{F_{Y i d e a l}}
$$

where $F_{Y}$ is the actual and $F_{Y i d e a l}$ is the ideal tangential force which is acting on 


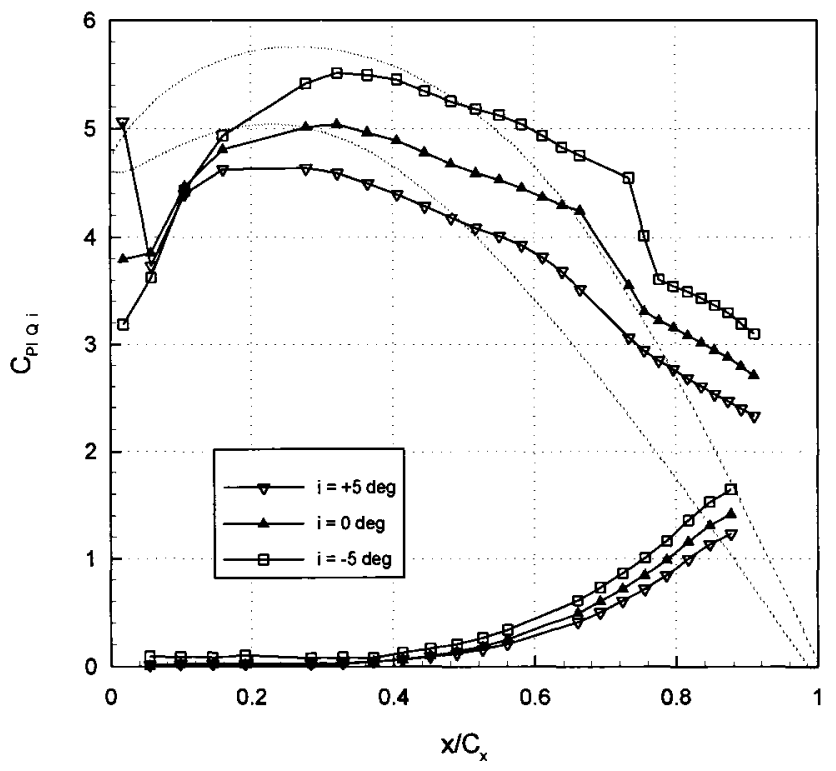

a) Loading Distributions Using $C_{P l Q i}$

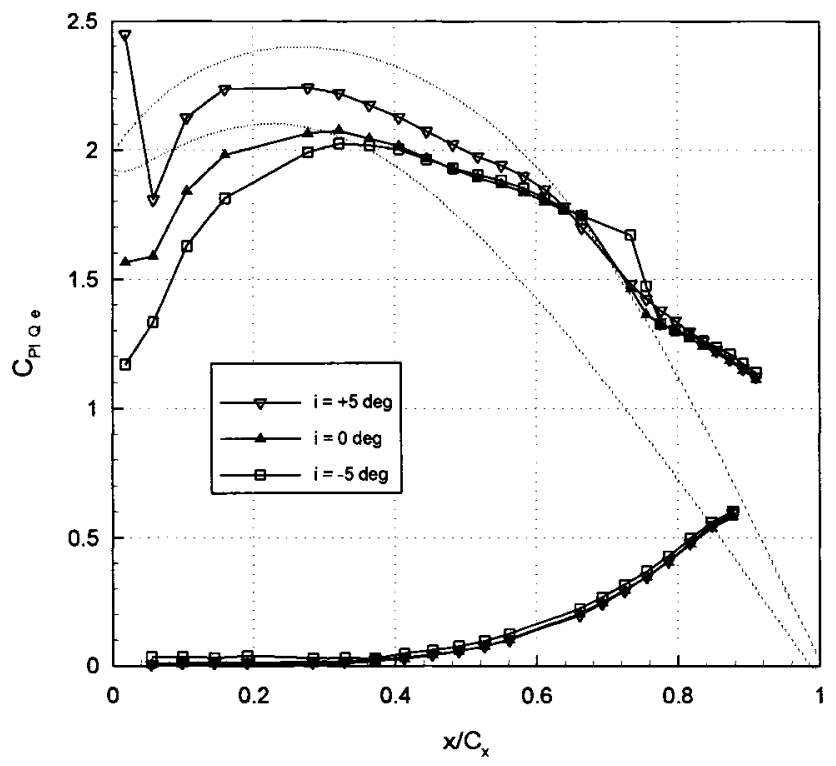

b) Loading Distributions Using $C_{P l Q e}$

Figure B.2: PAKD-F: Loading Distributions at $\mathrm{Re}=\mathbf{8 0 , 0 0 0}$ and FSTI $=\mathbf{1 . 5 \%}$ 


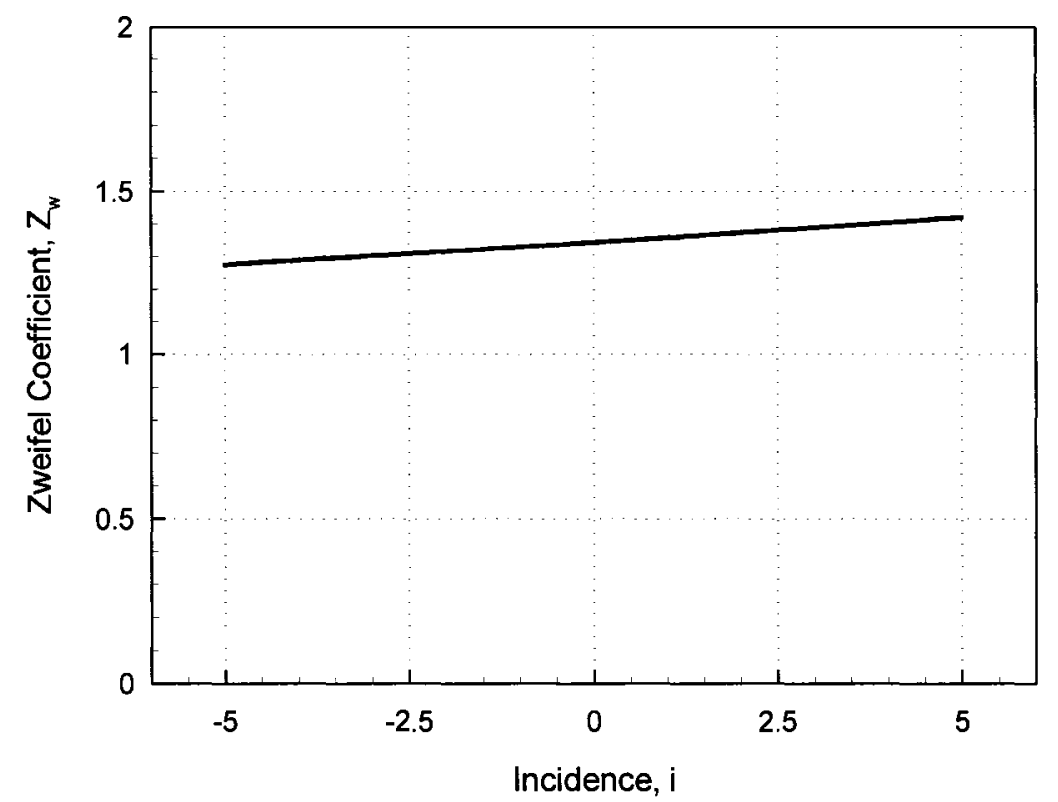

Figure B.3: PAKD-F: Zweifel Coefficient Variation with Incidence

the airfoil. Thus, the Zweifel coefficient is defined as the actual-to-ideal tangential force ratio can be expressed as:

$$
Z_{w}=\frac{\int_{0}^{C_{x}}\left(P_{P S}-P_{S S}\right) d x}{1 / 2 \rho U_{e}^{2} C_{x}}
$$

From the definition of $C_{P l Q e}$, it can be shown that:

$$
P_{P S}-P_{S S}=\frac{1}{2} \rho U_{e}^{2}\left(C_{P l, S S}-C_{P l, P S}\right)
$$

and thus the actual tangential force can be expressed as:

$$
Y=C_{x} \int_{0}^{1}\left(P_{S S}-P_{P S}\right) d\left(\frac{x}{C_{x}}\right)
$$




$$
Y=C_{x} \frac{1}{2} \rho U_{e}^{2} \int_{0}^{1}\left(C_{P l, S S}-C_{P l, P S}\right) d\left(\frac{x}{C_{x}}\right)
$$

where $C_{P l, S S}$ and $C_{P l, P S}$ are the suction side and pressure side static pressure coefficients based on the outlet dynamic pressure, respectively. Substituting into Eq. B.3, the Zweifel coefficient reduces to:

$$
Z_{w}=\int_{0}^{1}\left(C_{P l, S S}-C_{P l, P S}\right) d\left(\frac{x}{C_{x}}\right)
$$

which is the area enclosed by the curves on a plot of $C_{P l Q e}$ versus $x / C_{x}$ (as shown in Figure B.2(b).

The following can be concluded from the above discussion:

- The first definition of loading (based on inlet dynamic pressure) is useful for presenting the data with variation in Reynolds number at a fixed incidence.

- The second definition of pressure coefficient provides the correct visual impression of the changes in Zweifel coefficient for off-design incidence measurements. 


\section{Appendix C}

\section{Time Variation of Losses for Unsteady Flow Measurements}

This appendix will provide the instantaneous, time varying losses over the threewake passing periods for both PAKD-A and PAKD-F for all cases examined in the present work.

Figure C.1 shows the losses for both airfoils at the lowest reduced frequency examined $(f=0.5)$. Two dashed lines correspond to the time-averaged mixedout total losses, $Y_{\text {total }}$, and profile (mixed-out) losses, $Y_{m}$ as defined in Chapter 4 (Equations 4.25 and 4.26 , respectively). The difference between $Y_{\text {total }}$ and $Y_{m}$, is attributed to the rotating bar loss.

Figures C.2 to C.6 show the plots corresponding to reduced frequency from 1.0 to 3.0. 

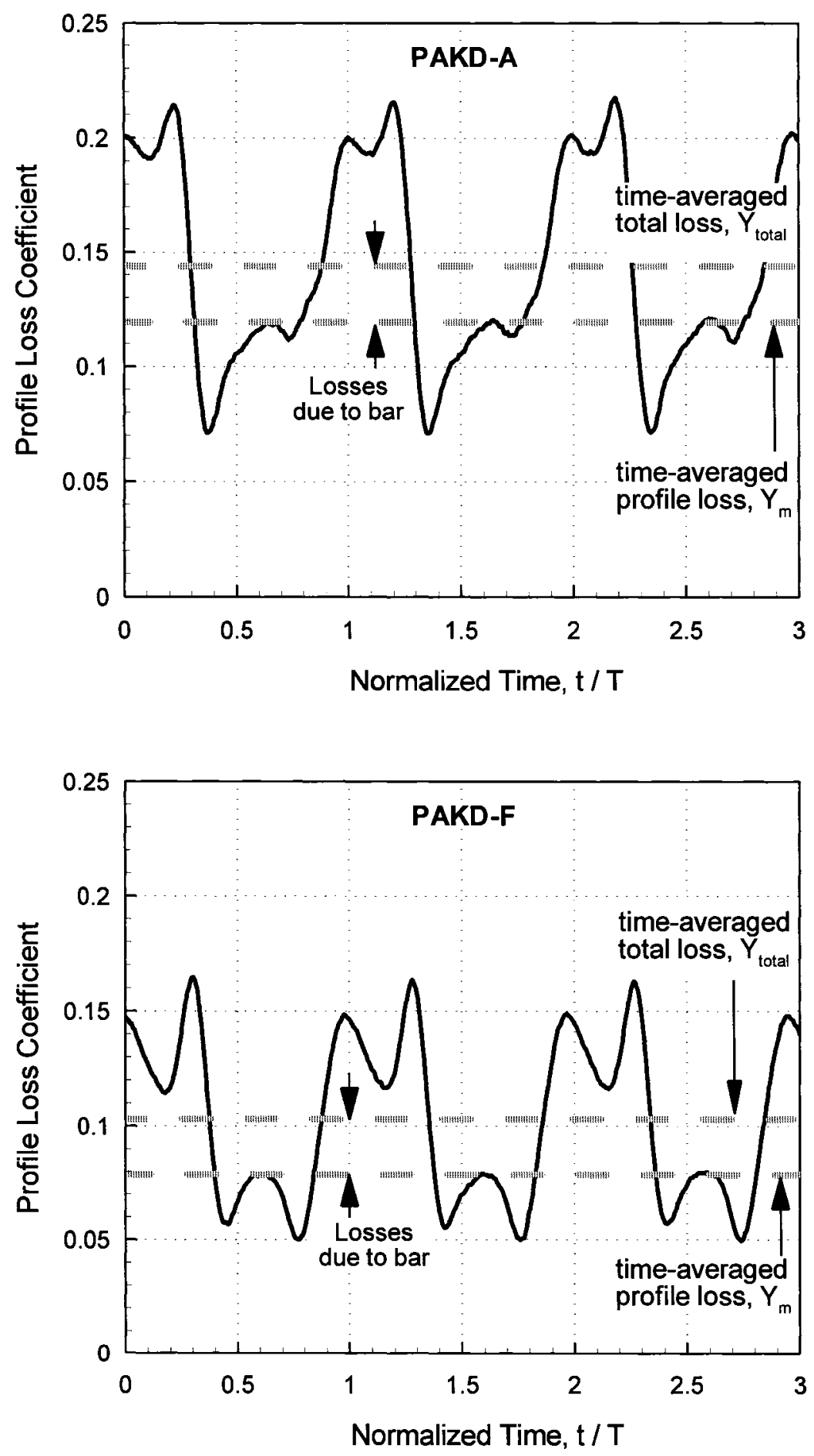

Figure C.1: Instantaneous Losses for $\mathrm{f}=\mathbf{0 . 5}$

$$
(\operatorname{Re}=50,000 \text { and } \mathrm{FSTI}=4 \%)
$$



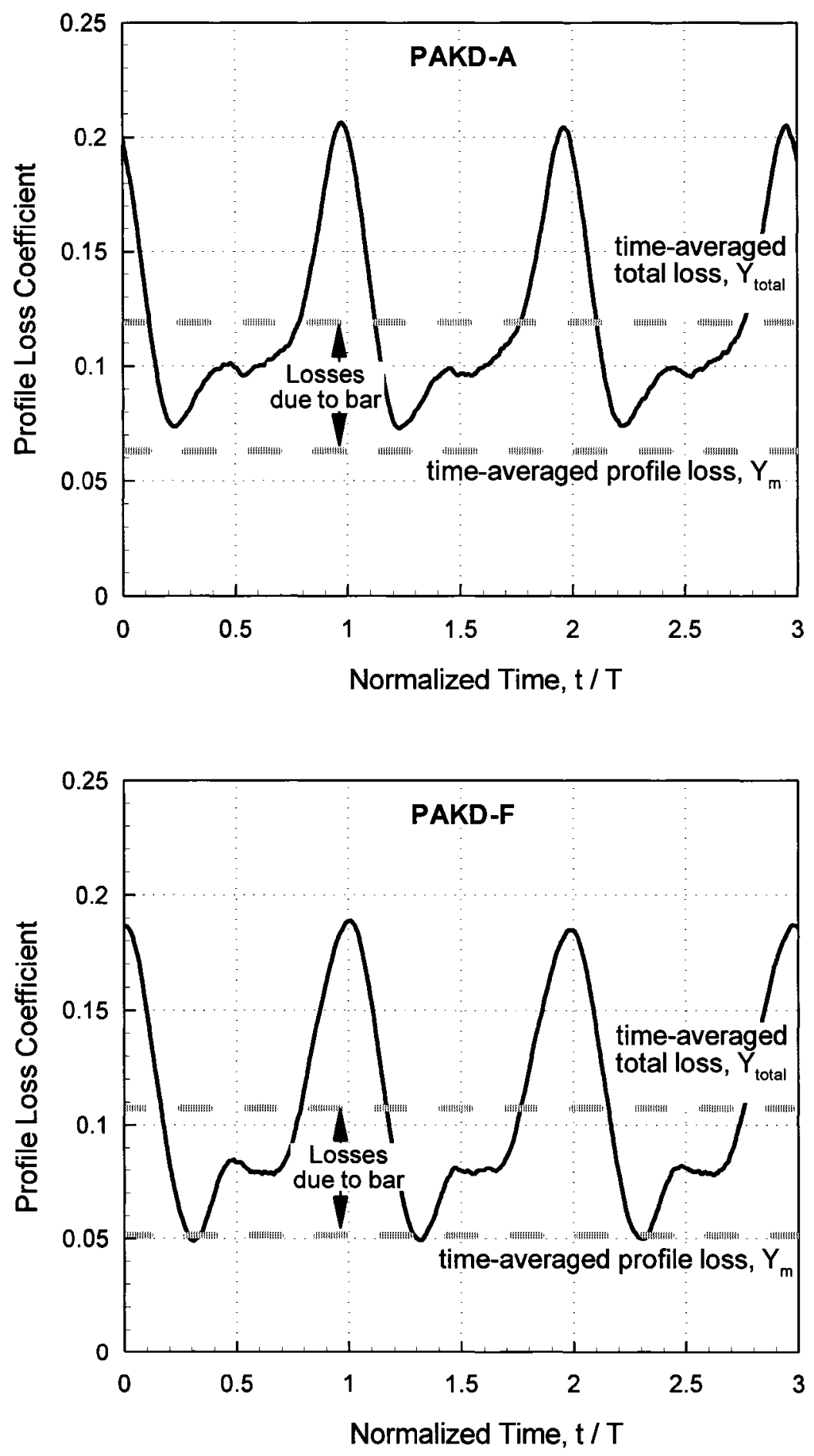

Figure C.2: Instantaneous Losses for $\mathrm{f}=\mathbf{1 . 0}$

$$
(\mathrm{Re}=50,000 \text { and } \mathrm{FSTI}=4 \%)
$$



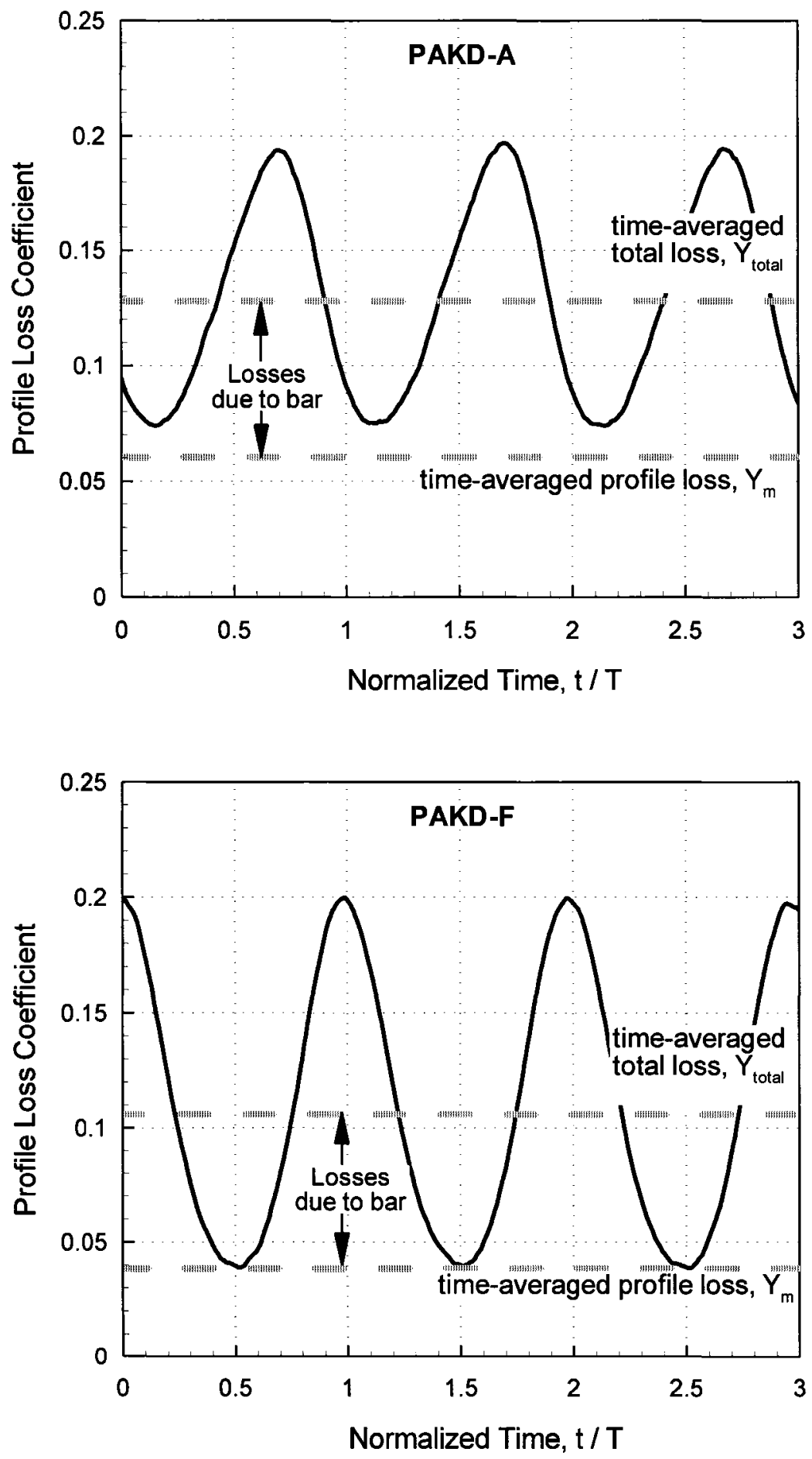

Figure C.3: Instantaneous Losses for $\mathrm{f}=\mathbf{1 . 6}$

$$
(\operatorname{Re}=50,000 \text { and } \mathrm{FSTI}=4 \%)
$$



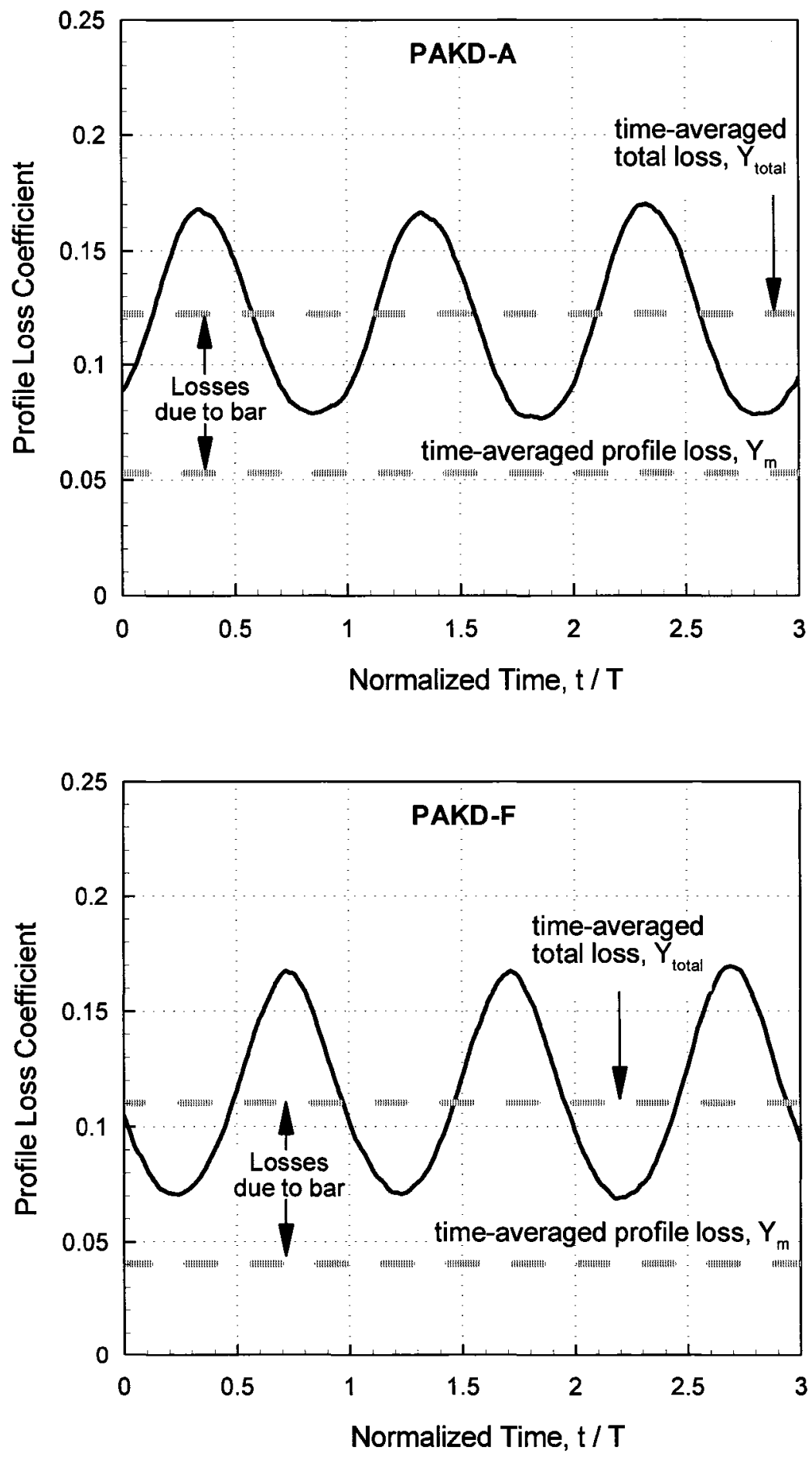

Figure C.4: Instantaneous Losses for $\mathbf{f}=\mathbf{2 . 0}$

$$
(\mathrm{Re}=50,000 \text { and } \mathrm{FSTI}=4 \%)
$$



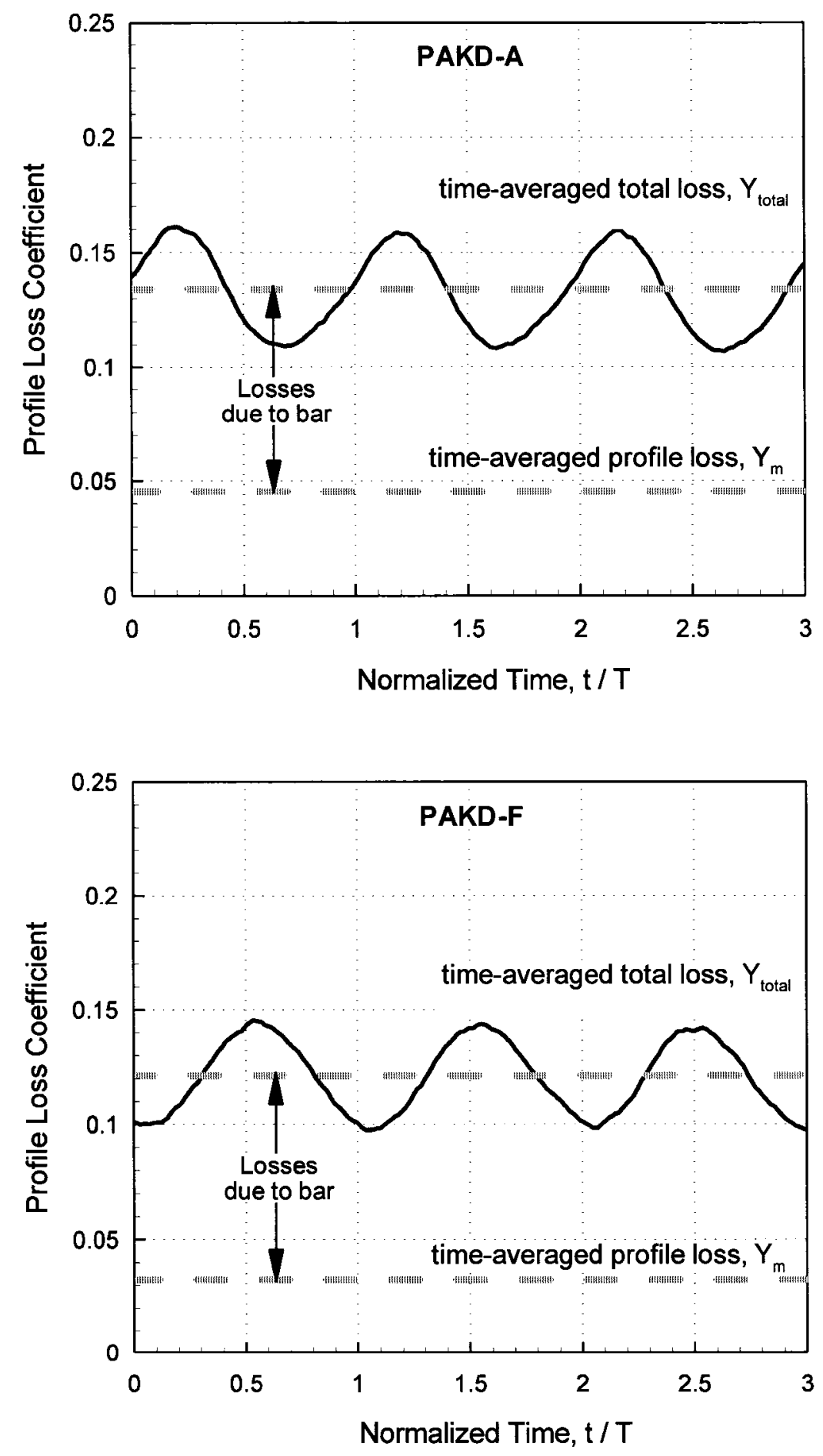

Figure C.5: Instantaneous Losses for $\mathbf{f}=\mathbf{2 . 5}$

$$
(\operatorname{Re}=50,000 \text { and FSTI }=4 \%)
$$



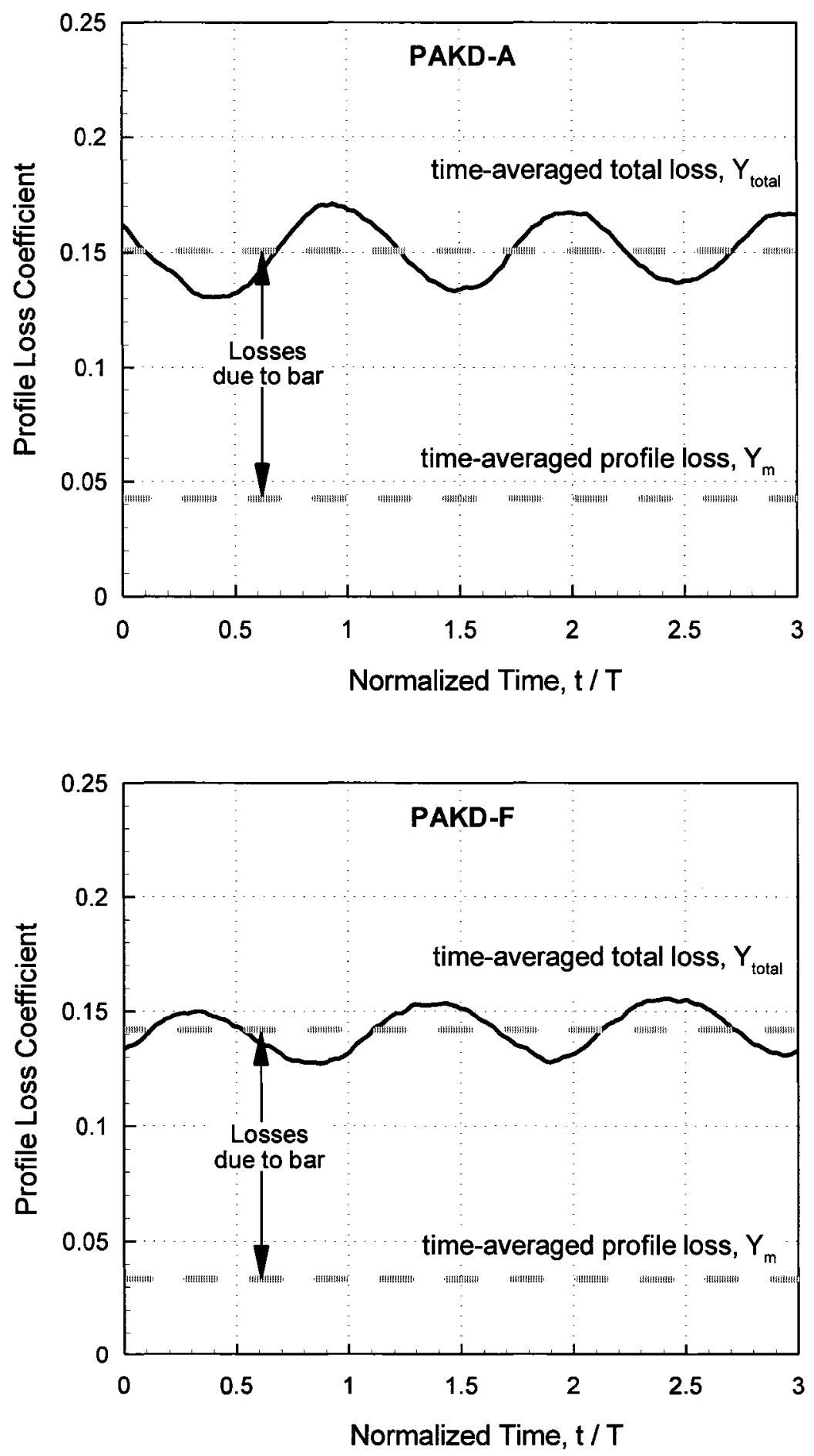

Figure C.6: Instantaneous Losses for $\mathbf{f}=\mathbf{3 . 0}$

$$
(\operatorname{Re}=50,000 \text { and } \mathrm{FSTI}=4 \%)
$$

